\title{
Contribuciones y análisis normativo para la integración de la energía eólica en sistemas de energía eléctrica
}

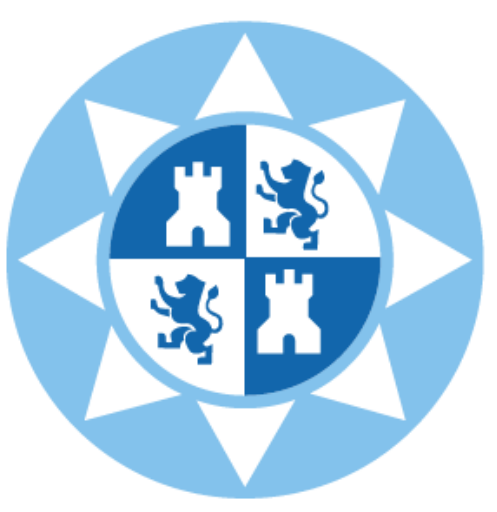

TESIS DOCTORAL

\begin{abstract}
Andrés Honrubia Escribano
Departamento de Ingeniería Eléctrica

Escuela Técnica Superior de Ingeniería Industrial

Universidad Politécnica de Cartagena
\end{abstract}

Octubre 2012 



\section{Contribuciones y análisis normativo para la integración de la energía eólica en sistemas de energía eléctrica}

Autor

Andrés Honrubia Escribano

Dirigida por los Doctores

Emilio Gómez Lázaro

Ángel Molina García

Departamento de Ingeniería Eléctrica

Escuela Técnica Superior de Ingeniería Industrial

Universidad Politécnica de Cartagena

Octubre 2012 



\section{Universidad}

Politécnica

de Cartagena

DT-16

\section{CONFORMIDAD DE SOLICITUD DEAUTORIZACIÓN DE DEPÓSITO DE TESIS DOCTORAL POR EL/LA DIRECTOR/A DE LA TESIS}

D./Da . Emilio Gómez Lázaro y Ángel Molina García, Directores de la Tesis doctoral Contribuciones y análisis normativo para la integración de la energía eólica en sistemas de energía eléctrica

\section{INFORMA:}

Que la referida Tesis Doctoral, ha sido realizada por D/Da. Andrés Honrubia Escribano, dando mi conformidad para que sea presentada ante la Comisión de Doctorado, para ser autorizado su depósito.

La rama de conocimiento por la que esta tesis ha sido desarrollada es:

1. Ciencias

- Ciencias Sociales y Jurídicas

$\checkmark$ Ingeniería y Arquitectura

En Cartagena, a $\quad 16$ de octubre de 2012

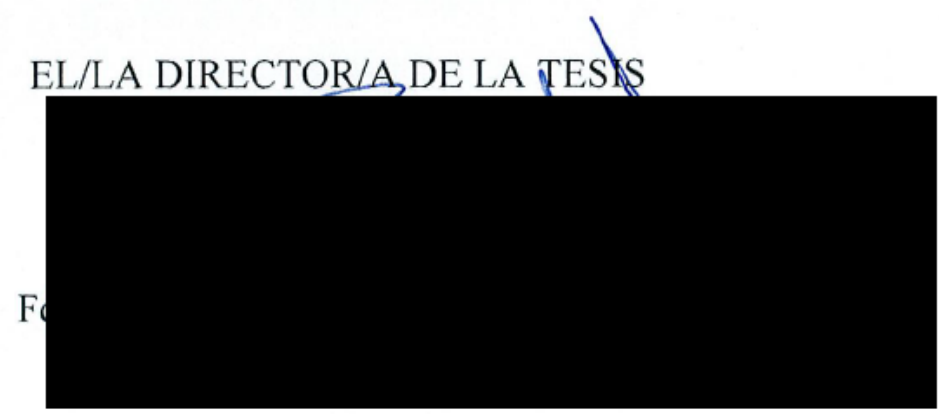





\section{CONFORMIDAD DE DEPÓSITO DE TESIS DOCTORAL POR LA COMISIÓN ACADÉMICA DEL PROGRAMA}

D/Da . José Ramón García Cascales, Presidente/a de la Comisión Académica del Programa ENERGÍAS RENOVABLES.

\section{INFORMA:}

Que la Tesis Doctoral titulada, "Contribuciones y análisis normativo para la integración de la energía eólica en sistemas de energía eléctrica", ha sido realizada por D/Da . Andrés Honrubia Escribano, bajo la dirección y supervisión del Dr. Emilio Gómez Lázaro y el Dr. Ángel Molina García.

En reunión de la Comisión Académica de fecha 25/Q2/12, visto que la mencionada tesis doctoral tiene acreditados los indicios de calidad, requeridos para el depósito de tesis doctorales, regulados en el artículo 32 del Reglamento de Estudios Oficiales de Máster y Doctorado de la UPCT, y la autorización del Director de la misma, se acordó dar la conformidad para que a dicha tesis le sea autorizado, por la Comisión de Doctorado, su depósito.

La Rama de conocimiento por la que esta tesis ha sido desarrollada es:

$\square$ Ciencias

$\square$ Ciencias Sociales y Jurídicas

Angeniería y Arquitectura

En Cartagena, a 4 -1 -de octubre de 2012

EL PRESIDENTE DE LA COMISIÓN ACADÉMICA DEL PROGRAMA

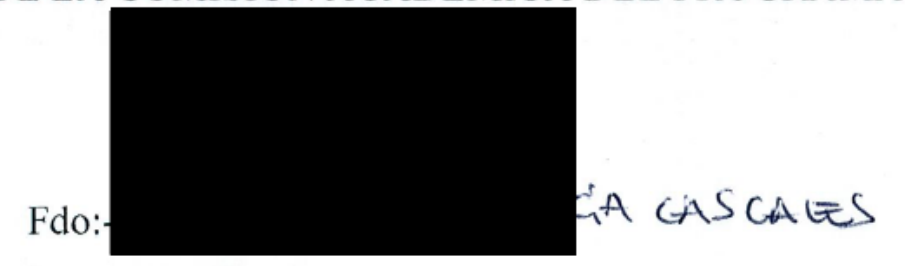



La inspiración existe, pero tiene que encontrarse trabajando Pablo Ruiz Picasso 



\section{Agradecimientos}

Tras varios años de estudio y aprendizaje siento que este largo camino no podría haberlo recorrido sin el apoyo y la ayuda de todas aquellas personas que han estado a mi lado. Resulta imposible nombrar a todas, así que voy a intentar ser breve.

A mis directores de Tesis, en especial a Emilio Gómez Lázaro que, con su conocimiento y experiencia ha sabido guiarme, depositando en mí una gran confianza; y a Ángel Molina García, por su forma de ser y su capacidad de motivación.

Por supuesto, agradecer a mis compañeros de trabajo, por compartir sus enseñanzas y consejos, y hacer del día a día un lugar ameno en el que trabajar y compartir experiencias personales.

Especial agradecimiento merece mi familia, sin cuyo apoyo y perseverancia no habría conseguido el último escalón académico y el mejor regalo posible, el orgullo de unos padres que hacen todo por sus hijos.

A mis amigos, por los buenos momentos que pasamos. 
Y, finalmente, pero no por ello menos importante, a mi novia, Lourdes, por estar siempre a mi lado.

Gracias a todos. 


\section{Resumen}

La energía eólica representa una de las energías de origen renovable con mayor implantación en nuestros días. Este hecho se debe, en gran parte, a los avances en tecnología de aerogeneradores y sistemas auxiliares. En paralelo al aumento de la capacidad de generación eólica, surge la necesidad de desarrollar normativas que regulen este sector energético emergente. De hecho, debido a que los aspectos regulatorios se encuentran en constante actualización, se ha decidido realizar la presente Tesis Doctoral abordando diferentes ámbitos normativos.

En primer lugar, cabe resaltar que la investigación aplicada sobre ensayo de curvas de potencia de aerogeneradores se encuentra en pleno desarrollo. Todavía hoy en la Norma Internacional vigente relativa a la caracterización de la curva de potencia de aerogeneradores, la velocidad del viento a la altura del buje es el parámetro principal de entrada para la caracterización de dicha curva. Esta suposición implica que la velocidad del viento es constante en toda el área barrida del rotor. Sin embargo, el diámetro del rotor y la altura de los aerogeneradores presentes en el mercado actual son considerablemente mayores de los que existían cuando se publicó dicha Norma en el año 2005. Por este motivo, actualmente existe un Comité Técnico encargado de la actualización de esta Norma, donde se han vertido parte de las contribuciones y aportaciones de esta Tesis.

Por otro lado, y con el objeto de estandarizar la gran variedad de modelos de simulación de aerogeneradores que existe en el sector, a finales del año 2009 se creó un Comité Técnico dedicado al desarrollo de modelos simplificados de aerogeneradores y parques eólicos, en el que uno de los Directores de la presente Tesis es el responsable del Comité Español. Dicho Comité Técnico se encuentra actualmente inmerso en el desarrollo de una Norma en la que se están definiendo modelos de aerogeneradores capaces de ser integrados en estudios de estabilidad de los sistemas eléctricos ante grandes perturbaciones. Esta Tesis ha participado con sus aportaciones en los primeros pasos que se han dado en este ámbito. 
En tercer lugar, y debido a la sensibilidad de la aparamenta eléctrica y de los componentes electrónicos frente a perturbaciones, la evaluación de su comportamiento en sistemas eléctricos en general y en parques eólicos en particular resulta ser un tema de elevado interés en la actualidad. Más aún cuando la normativa actual requiere de un mayor conocimiento sobre el comportamiento eléctrico de estos componentes, así como las variables más influyentes en el mismo. En esta línea, la presente Tesis incluye una amplia variedad de ensayos realizados sobre diverso equipamiento eléctrico presente en instalaciones de generación de energía eólica ante las perturbaciones más habituales.

En suma, la presente Tesis Doctoral contribuye a resolver las cuestiones normativas descritas en los párrafos anteriores, al mismo tiempo que analiza su influencia en la integración de la energía eólica en los sistemas eléctricos. 


\section{Abstract}

Nowadays, wind energy represents one of the renewable energy sources with the largest establishment. This development is largely due to the advancement in wind turbine technology and ancillary systems. In parallel with the increase of the wind power capacity, standards to control this emerging energy sector are needed. Since these normalisation concerns results in a constant update, it has been decided to perform the present Doctoral Thesis dealing with several regulatory aspects.

First of all, it should be pointed out that the research related to power curve performance of wind turbines is in continuous development. Even today in the current edition of the International Standard related to power curve performance, the wind speed at hub height is the primary input parameter for power curve measurements. This assumption implies that wind speed is constant over the whole turbine swept rotor area. However, both rotor diameter and hub height of modern wind turbines are significantly large in comparison with the wind turbines developed in 2005, when the Standard was issued. For this reason, a Technical Committee has recently been created devoted to update this Standard, where some of the contributions of this Thesis are included.

Secondly, with the aim of standardizing the huge diversity of simulation models for wind turbines present in the wind energy sector, at the end of 2009 a Technical Committee focused on the development of generic models for both wind turbines and wind farms was created. The national responsible of this Committee supervises this Thesis. Currently, this Technical Committee is developing an International Standard where wind turbine models for the integration of wind power generation in studies of large-disturbance power system stability are defined. This Thesis has collaborated towards the first steps for the development of this Standard.

In the third place and due to the sensitivity of electrical equipment and electronic components to electromagnetic disturbances, nowadays the assessment of their behavior in power systems in general and in wind farms in par- 
ticular represents an interesting issue. Furthermore, both a higher knowledge and the most influential parameters are required by the current standards. From this point of view, the present Thesis includes a wide variety of tests carried out over different electrical equipment commonly used in wind farms under most typical electromagnetic disturbances.

Taking into account these considerations, the present Doctoral Thesis contributes to the solution of the regulatory concerns pointed out in the preceding paragraphs, together with an analysis of their influence on the integration of wind energy in power systems. 


\section{Índice}

\begin{tabular}{ll}
\hline Agradecimientos & XI
\end{tabular}

Resumen XIII

Abstract $\quad$ XV

1. Introducción y Antecedentes 1

1.1. Situación Actual de la Energía Eólica . . . . . . . . . . . . . 1

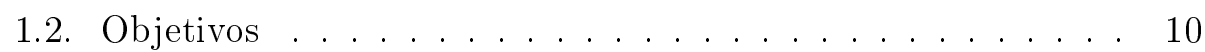

1.3. Estructura de la Tesis . . . . . . . . . . . . . . . . . 11

2. Equipamiento eléctrico y huecos de tensión 13

2.1. Calidad del suministro eléctrico . . . . . . . . . . . . . . 13

2.1.1. Huecos de tensión e interrupciones . . . . . . . . . 16

2.1.2. Curvas de tolerancia . . . . . . . . . . . . . 19

2.2. Análisis del equipamiento ensayado . . . . . . . . . . . 24

2.2.1. Análisis del Contactor . . . . . . . . . . . . . . . 24

2.2.2. Análisis del relé de falta a tierra . . . . . . . . . . 34

2.2.3. Análisis del variador de frecuencia . . . . . . . . . . 35

2.2.4. Análisis del ordenador personal . . . . . . . . . . . . 45

2.2.5. Análisis de diversas cargas de iluminación . . . . . . . 51

2.3. Descripción de los ensayos y del material empleado . . . . . . 56

2.3.1. Generador de huecos de tensión . . . . . . . . . . . 56

2.3.2. Características del equipamiento ensayado . . . . . . . 57

2.3.3. Descripción del procedimiento de ensayo . . . . . . . . 62

2.4. Resultados . . . . . . . . . . . . . . . 65

2.4.1. Ensayos realizados sobre contactores . . . . . . . . 65

2.4.2. Ensayos realizados con el relé de falta a tierra . . . . . 87

2.4.3. Ensayos realizados con el variador de frecuencia . . . . 88 
2.4.4. Ensayos realizados con el ordenador personal . . . . . 90

$\begin{array}{lll}2.4 .5 . & \text { Ensayos realizados con diversas cargas de iluminación } 92\end{array}$

2.5. Conclusiones .................. 96

3. LiDAR y generación eólica 99

3.1. Introducción. . . . . . . . . . . . . . . . . . 99

3.1.1. El viento. Nociones generales sobre el gradiente de viento 101

3.1.2. Sistemas de medición del recurso eólico. . . . . . . . . 108

3.1.3. Curva de potencia. Velocidad equivalente . . . . . . . 119

3.1.4. Estado del arte . . . . . . . . . . . . . . . 121

3.2. Descripción de los emplazamientos . . . . . . . . . . . . . . 127

3.2.1. Parque eólico 1 . . . . . . . . . . . . . . . . . . . 127

3.2.2. Parque eólico 2 . . . . . . . . . . . . . . . . . . 129

3.2.3. Parque eólico 3 . . . . . . . . . . . . . . 133

3.3. Resultados. . . . . . . . . . . . . . . . . 138

3.3.1. Tratamiento de la información . . . . . . . . . . . . 138

3.3.2. Análisis del gradiente de viento . . . . . . . . . . . . . 139

3.3.3. Evaluación de la respuesta del equipo LiDAR frente a sistemas convencionales . . . . . . . . . . 148

3.3.4. Influencia del gradiente de viento en la generación de energía eólica . . . . . . . . . . . . . 164

3.4. Conclusiones . . . . . . . . . . . . . . . . . 173

4. Modelos simplificados de aerogeneradores $\quad 177$

4.1. Introducción . . . . . . . . . . . . . . . . . 177

4.1.1. Antecedentes de la IEC 61400-27 . . . . . . . . . . . . 179

4.2. Descripción de los ensayos y del material empleado . . . . . . 184

4.3. Desarrollo de modelos simplificados de aerogeneradores . . . 187

4.3.1. Modelo aerodinámico . . . . . . . . . . . . . . . 188

4.3.2. Modelo mecánico . . . . . . . . . . . . . . . . . . 196

4.3.3. Modelo de control de pitch . . . . . . . . . . . . . 200

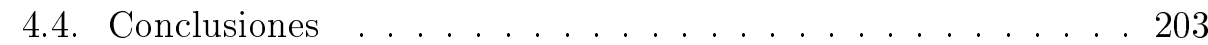

5. Conclusiones, aportaciones y trabajos futuros 205

5.1. Conclusiones generales . . . . . . . . . . . . . . 205

5.1.1. Equipamiento eléctrico y huecos de tensión . . . . . . 206

5.1.2. LiDAR y generación eólica. . . . . . . . . . . . . . . . 207

5.1.3. Modelos simplificados de aerogeneradores . . . . . . 208

5.2. Aportaciones . . . . . . . . . . . . . . 209

5.3. Trabajos futuros .................. . 210

\begin{tabular}{ll}
\hline Bibliografía & 213
\end{tabular} 



\section{Índice de figuras}

1.1. Evolución de la potencia eólica instalada anual, acumulada y tasa de variación en España, $\operatorname{AEE}[(2012)$. . . . . . . . . . . . 2

1.2. Distribución del tamaño de los aerogeneradores instalados en el año 2011 en España, $\operatorname{AEE} \mid(2012)$.$] . . . . . . . . . . . . . . 3$

1.3. Distribución de la potencia eólica instalada en el año 2011 en el mundo, GWEC $[(2012)$. . . . . . . . . . . . . . . . 4

1.4. Evolución de la potencia eólica instalada anualmente en el mundo, $\operatorname{AEE}[(2012)$.]. . . . . . . . . . . . . . . . . 4

1.5. Evolución del tamaño de los aerogeneradores, EWEA (2009). 5

1.6. Equipo LiDAR (delante) junto a una torre meteorológica (detrás) en un parque eólico, Honrubia et al.[(2010d).] . . . . . . 6

1.7. Cronograma estimado de desarrollo de la IEC 61400-27, Sørensen (2012)....................... . . . . 9

2.1. Definición de los parámetros de un hueco de tensión. . . . . . 17

2.2. Diferentes tipologías de huecos de tensión. . . . . . . . . . . . 18

2.3. Curva ITI. . . . . . . . . . . . . . . . . . . . . . . . . . 21

2.4. Comparativa de las curvas de tolerancia. . . . . . . . . . 22

2.5. Imagen 3D de un contactor de corriente continua, Riba et al.

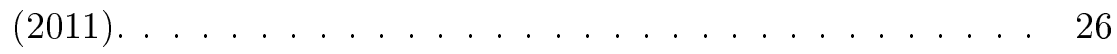

2.6. Esquema de un contactor de corriente alterna. . . . . . . . . . 26

2.7. Sistema de protección por relé de falta a tierra. . . . . . . . . 35

2.8. Esquema constructivo de un variador de frecuencia. . . . . . . 36

2.9. Variación de la velocidad de un motor en función del hueco de tensión aplicado sobre el variador de frecuencia, Rocha y Madrigal (2005). . . . . . . . . . . . . . . . . . 38

2.10. Comportamiento del variador de frecuencia ante un hueco de tensión trifásico equilibrado, O’Connell y Kirawanich (2005). . 39 
2.11. Esquema constructivo de una fuente de alimentación conmu-

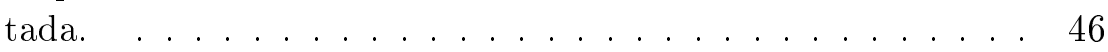

2.12. Vista frontal de la fuente de potencia. . . . . . . . . 57

2.13. Diagrama de flujo del procedimiento de ensayo diseñado. . . . 63

2.14. Ejemplo de ensayo experimental. . . . . . . . . . . 64

2.15. Curvas de tolerancia del contactor Schneider en vacío. . . . . 68

2.16. Curvas de tolerancia del contactor Telemecanique en vacío. . 69

2.17. Curvas de tolerancia del contactor Mitsubishi en vacío. . . . . 70

2.18. Curvas de tolerancia del contactor Telemecanique alimentando diversas cargas resistivas. . . . . . . . . . . . . 73

$\begin{array}{ll}\text { 2.19. Curvas de tolerancia del contactor Schneider. Carga inductiva. } & 75\end{array}$

2.20. Curvas de tolerancia del contactor Telemecanique. Carga in-

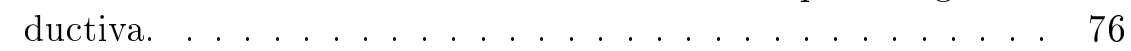

2.21. Curvas de tolerancia del contactor Mitsubishi. Carga inductiva. 77

2.22. Curvas de tolerancia del contactor Schneider. Carga inductiva en paralelo. . . . . . . . . . . . . . . . 79

2.23. Curvas de tolerancia del contactor Telemecanique. Carga inductiva en paralelo. . . . . . . . . . . . . 80

2.24. Curvas de tolerancia del contactor Mitsubishi. Carga inductiva en paralelo. . . . . . . . . . . . . . 81

2.25. Curvas de tolerancia del contactor Schneider. Carga capacitiva. 83

2.26. Curvas de tolerancia del contactor Telemecanique. Carga capacitiva. ....................... 84

2.27. Curvas de tolerancia del contactor Mitsubishi. Carga capacitiva. 85

2.28. Influencia de la temperatura en la respuesta del contactor. . . 86

2.29. Curva de tolerancia del relé de falta a tierra. . . . . . . . . 88

2.30. Curva de tolerancia del variador de frecuencia. . . . . . . . 89

2.31. Detalle del comportamiento del variador de frecuencia. . . . . 91

2.32. Curva de tolerancia del ordenador personal. . . . . . . . . . 92

2.33. Intensidad absorbida por el ordenador personal cuando se recupera la tensión nominal. . . . . . . . . . . . . . . 93

2.34. Curvas de tolerancia de diversas cargas de iluminación. . . . . 94

3.1. Origen del viento, Esteban Pérez [2009). . . . . . . . . . . . . 102

3.2. Capa límite terrestre. . . . . . . . . . . . . . . . . . 103

3.3. Variación de la velocidad del viento con la altura según Ley Potencial. . . . . . . . . . . . . 106

3.4. Comparativa Leyes de predicción del gradiente de viento, Honrubia et al.[(2010d). . . . . . . . . . . . . . . 107

3.5. Anemómetro de cazoletas Thies First Class Advanced. . . . . 109

3.6. Anemómetro de hélice Young Compnay. . . . . . . . . . . . . 111

3.7. Anemómetro sónico WindMaster Pro. . . . . . . . . . . . . . 113 


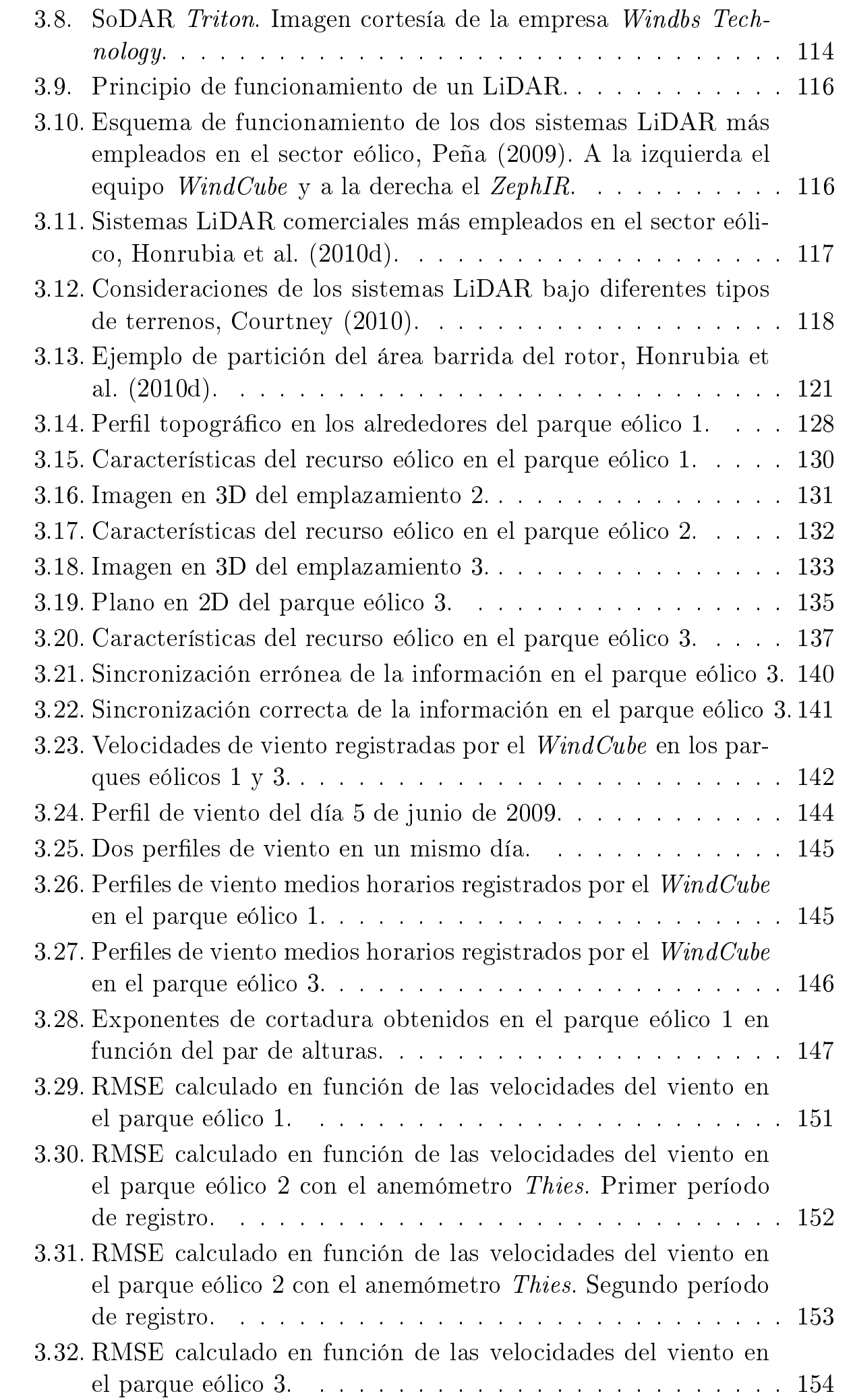


3.33. RMSE calculado en función de las direcciones del viento en el parque eólico 1. . . . . . . . . . . . . . . . . . 155

3.34. RMSE calculado en función de las direcciones del viento en el parque eólico 2, anemómetro Thies. Primer período de registro. 156

3.35. RMSE calculado en función de las direcciones del viento en el parque eólico 2, anemómetro Thies. Segundo período de registro. 157

3.36. RMSE calculado en función de las direcciones del viento en el

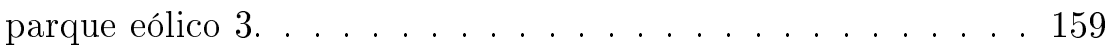

3.37. RMSE calculado en función de las velocidades del viento en el parque eólico 3, aplicando filtro de direcciones de viento. . 160

3.38. Correlación de la velocidad del viento en función de direcciones del viento en el parque eólico 1. . . . . . . . . . . . . . . . 161

3.39. Correlación de la velocidad del viento filtrada en el parque eólico 3. . . . . . . . . . . . . . . . . 162

3.40. Correlación de la desviación estándar de la velocidad del viento en el parque eólico 1. . . . . . . . . . . . . . . . . . . 163

3.41. Correlación de la dirección del viento en el parque eólico 1. . 164

3.42. Curvas de potencia de los nueve aerogeneradores del parque eólico 3. . . . . . . . . . . . . . . 166

3.43. RMSE entre LiDAR y aerogenerador 2 del parque eólico 3 en función de las direcciones del viento registradas por el Wind-

Cube con diferentes filtros de velocidades. . . . . . . . . . 167

3.44. Área barrida del aerogenerador 2 dividida en 6 secciones. . . . 168

3.45. Curvas de potencia del aerogenerador 2. . . . . . . . . 168

3.46. Área barrida del aerogenerador 2 dividida en 6 secciones idénticas. . . . . . . . . . . . . . . 170

3.47. Curvas de potencia del aerogenerador 2. Velocidades equivalentes obtenidas mediante criterio de igualdad de áreas. . . . . 170

3.48. Curva de potencia del aerogenerador 2 en función del coeficiente de cortadura, $\alpha . \ldots \ldots \ldots \ldots 171$

4.1. Tipologías de aerogeneradores más empleadas. . . . . . . . . . 182

4.2. Instalación de los analizadores de calidad de energía eléctrica $\begin{array}{lll}\text { dentro de la góndola del aerogenerador } 4 \text { del parque eólico } 3 . & 184\end{array}$

4.3. Ejemplo de magnitudes registradas por los equipos instalados en el parque eólico 3 durante un hueco de tensión. . . . . . . . 186

4.4. Diagrama de bloques general de los modelos simplificados. . . 187 \begin{tabular}{l} 
4.5. Representación tridimensional de $C_{p}$, Price y Sanchez-Gasca \\
\hline$(2006) . \ldots \ldots \ldots \ldots$ \\
\hline
\end{tabular}

4.6. $C_{p}$ en función de $\lambda$ y $\theta$, Fortmann $[(2010)$. . . . . . . . . . . . 189

4.7. Representación del término $\frac{\partial p}{\partial \omega_{\text {rotor }}}$, Fortmann $(2010)$. . . . . . 192 
4.8. Variación del ángulo de pitch de un aerogenerador en función de la velocidad del viento registrada en el parque eólico 3. . . 194

4.9. Implementación del modelo aerodinámico simplificado. . . . . 195

4.10. Esquema general de un modelo mecánico de dos masas. . . . 197

4.11. Esquema de bloques del modelo mecánico. . . . . . . . . . . 199

4.12. Esquema de bloques del modelo de control de pitch. . . . . . 201 



\section{Índice de Tablas}

2.1. Tipos de perturbaciones de tensión de corta duración según IEEE Std. 1159-2009]. . . . . . . . . . . . . . . . . . . . 16

2.2. Categorías de empleo de contactores, UNE-EN 60947-4-1.] . . 30

2.3. Resumen de los principales estudios realizados sobre huecos de tensión en contactores con diferentes ángulos de inicio de la onda de tensión. . . . . . . . . . . . . . . . . . . 33

2.4. Resumen de los principales estudios realizados sobre huecos de tensión en variadores de frecuencia. . . . . . . . . . . . . . 44

2.5. Resumen de los principales estudios realizados sobre huecos de tensión en ordenadores personales. . . . . . . . . . . . . . 50

2.6. Resumen de los principales estudios realizados sobre huecos de tensión en diversas cargas de iluminación. . . . . . . . . . 55

2.7. Características de los contactores ensayados. . . . . . . . . . . 59

2.8. Características del relé de falta a tierra ensayado. . . . . . . . 60

2.9. Características del variador de frecuencia ensayado. . . . . . . 60

2.10. Características del ordenador personal ensayado. . . . . . . . 61

2.11. Resumen de los ensayos realizados sobre los tres contactores en vacío. . . . . . . . . . . . . . . . . . . . . 71

2.12. Resumen de los ensayos realizados sobre huecos de tensión en variadores de frecuencia. . . . . . . . . . . . . . . . . 90

3.1. Cantidad de muestras no válidas en el parque eólico 1. . . . 129

3.2. Cantidad de datos disponible a cada altura de registro del LiDAR en el parque eólico 3. . . . . . . . . . . . . . . . . 136

3.3. Velocidades de viento medias registradas por el equipo LiDAR en los parques eólicos 1 y 3. . . . . . . . . . . . . . . . . . 143

3.4. RMSE calculado en el parque eólico 2. . . . . . . . . . . . . . 150

3.5. Sectores de dirección del viento utilizados. . . . . . . . . . . . 154 
4.1. Versiones comerciales de las topologías de aerogeneradores más comunes. . . . . . . . . . . . . . . . . . . . . . . 183

4.2. Valores de parámetros necesarios para calcular el término $\frac{\partial p}{\partial \theta}$. 191

4.3. Parámetros necesarios para calcular el término $\frac{\partial p}{\partial \omega_{\text {rotor }}} . \mid$. . . . 193

4.4. Valores de parámetros necesarios para calcular el término $\frac{\partial p}{\partial \omega_{\text {rotor }}} .193$

4.5. Parámetros necesarios para modelar el sistema mecánico. . . . 198

4.6. Parámetros necesarios para modelar el sistema de control de pitch. ..................... 202 
Capítulo 1

\section{Introducción y Antecedentes}

Resumen: El presente primer capítulo de la Tesis Doctoral pretende introducir la temática desarrollada, al igual que los objetivos planteados. Finalmente, se muestra la estructura que posee el documento. Para comenzar esta parte introductoria, se muestra un resumen del contexto actual de la energía eólica junto con las razones que han motivado la Tesis.

\subsection{Situación Actual de la Energía Eólica}

Actualmente, es innegable la importancia que está adquiriendo la energía eólica en el desarrollo de nuestra sociedad, convirtiéndose en uno de los pilares fundamentales de aportación de las energías renovables con un total de 22.307 MW de potencia eléctrica equivalente instalada a julio de 2012 (datos ofrecidos por la Comisión Nacional de la Energía, CNE (2012)), y representando así en torno al $20 \%$ de toda la potencia eléctrica instalada en nuestro país. Por Comunidades Autónomas, Castilla y León lidera la presencia de generación eólica con 5.277 MW, seguida por Castilla-La Mancha con 3.725 MW y Galicia con 3.299 MW.

No sólo en cuanto a potencia instalada, sino también en cuanto a energía producida, la energía eólica se sitúa en el nivel más alto de las energías renovables, cubriendo una media superior al $16 \%$ de toda la energía eléctrica demandada en nuestro país durante el año 2011; lo que supone ocupar el tercer lugar en cuanto a cobertura de la demanda, REE (2012). Incluso, en determinados momentos de los últimos meses, se ha dado la situación que más del $50 \%$ de toda la generación eléctrica que se estaba produciendo en el país era proporcionada por la eólica. Concretamente, consultando los datos ofrecidos por Red Eléctrica de España (REE), el día 19 de abril de 2011 a 


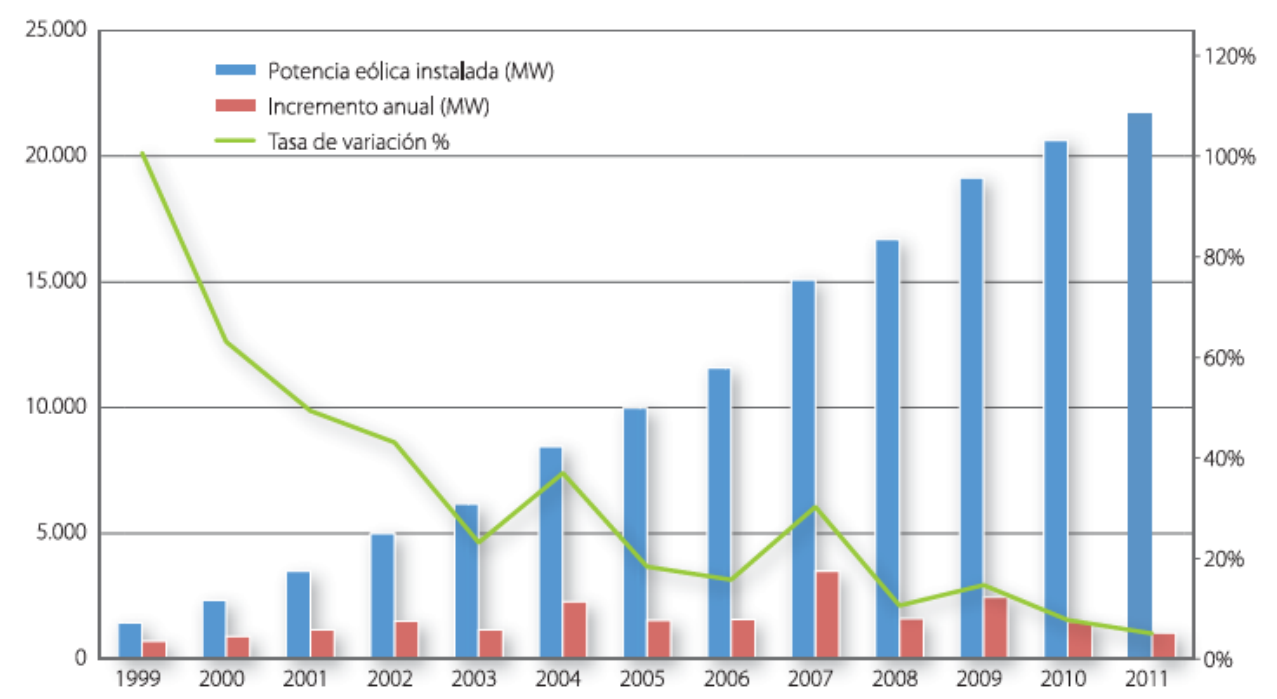

Figura 1.1: Evolución de la potencia eólica instalada anual, acumulada y tasa de variación en España, AEE 2012.

las 01:30 horas, la eólica contribuyó un $60 \%$ a la demanda eléctrica en ese momento.

Sin embargo, hay que señalar que, en el año 2011, en España solamente se instalaron 1.050 MW, lo que representa el crecimiento más débil de la historia del país en términos porcentuales. La figura 1.1 AEE (2012), muestra la evolución de la potencia instalada en España desde el año 1999, donde se observa la reducida tasa de variación sufrida en el año 2011 (línea verde de dicha figura). De hecho, existen ocho Comunidades Autónomas que no han instalado potencia alguna durante el año 2011.

En relación con el tamaño de los aerogeneradores, de las 581 unidades que se instalaron en España en 2011, más del $65 \%$ tienen una potencia unitaria igual o superior a $2 \mathrm{MW}$. Concretamente, de acuerdo con la figura $1.2 \mathrm{se}$ instaló 1 aerogenerador de $4.5 \mathrm{MW}, 56$ de $2.5 \mathrm{MW}, 21$ de $2.3 \mathrm{MW}$ y 304 de 2 MW, AEE (2012). Con ello, a finales de 2011, se contaba con 19.606 aerogeneradores instalados en España.

Es importante tener en cuenta que la inclinación hacia este tipo de energía que ha habido en España no ha sido la única en el mundo. En el año 2011 se produjo un aumento del $6 \%$ de la potencia eólica instalada en el mundo con respecto al año anterior, contribuyendo a una suma total muy próxima a 238 GW, GWEC (2012). Los principales impulsores de este crecimiento en el año 2011 se encuentran en Asia. Concretamente, China instaló el $43 \%$ del mercado y, junto con La India, suponen el $50 \%$ de toda la potencia eólica instalada a nivel mundial durante 2011. En este sentido, la figura 1.3 muestra, a nivel mundial, la distribución de la potencia eólica instalada en 


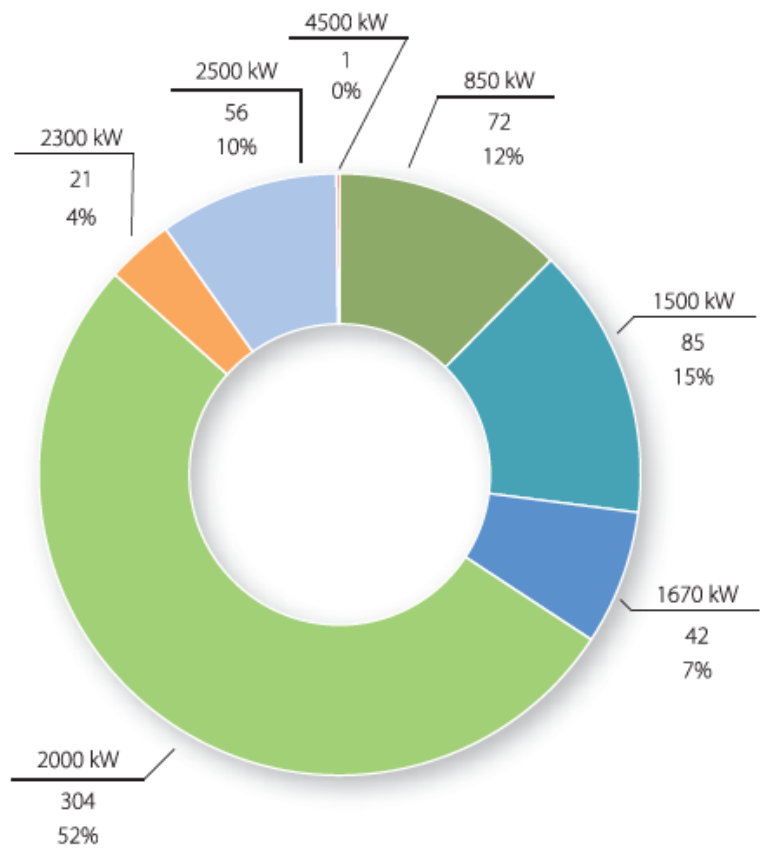

Figura 1.2: Distribución del tamaño de los aerogeneradores instalados en el año 2011 en España, AEE (2012).

el año 2011.

Según los datos manejados por las diferentes organizaciones, tanto nacionales como internacionales, relacionadas con el sector eólico, AEE (2012); GWEC (2012); WWEA (2012), hasta junio de 2012 sólo tres países (China, Estados Unidos y Alemania), se sitúan por delante de España en cuanto a potencia eólica instalada. La figura 1.4 muestra la potencia instalada a nivel mundial en MW, hasta junio de 2011. Un dato curioso que se puede extraer de la figura 1.4 es que China instaló en el año 2010 casi la misma cantidad de potencia eólica que España ha instalado a lo largo de toda su historia.

Con todo ello, dentro de este marco energético, hoy en día siguen existiendo cuestiones que no han sido resueltas con la precisión requerida. En estos momentos, la investigación aplicada sobre ensayo de curvas de potencia de aerogeneradores se encuentra en pleno desarrollo. Concretamente, la curva de potencia de un aerogenerador proporciona la relación entre la velocidad de viento incidente sobre el rotor eólico con la potencia generada por el aerogenerador. Todavía en la Norma Internacional vigente relativa a la caracterización de la curva de potencia de aerogeneradores, (International Electrotechnical Commission 2005), la velocidad del viento a la altura del buje es el parámetro principal de entrada para la caracterización de dicha curva, junto al parámetro densidad del aire como factor secundario.

La suposición anterior implica que la velocidad del viento es constante en 


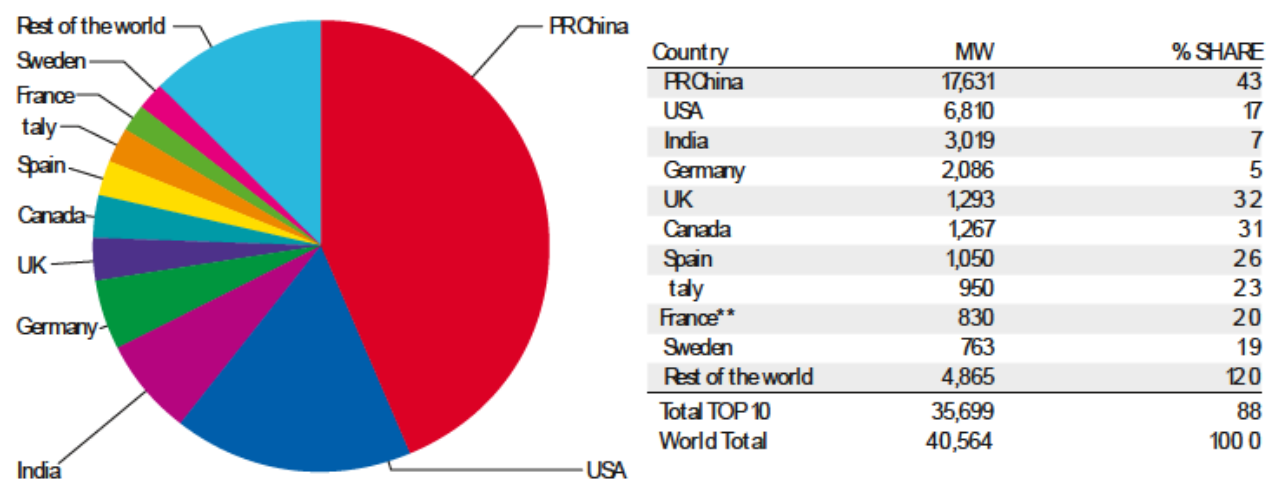

Figura 1.3: Distribución de la potencia eólica instalada en el año 2011 en el mundo, GWEC (2012).

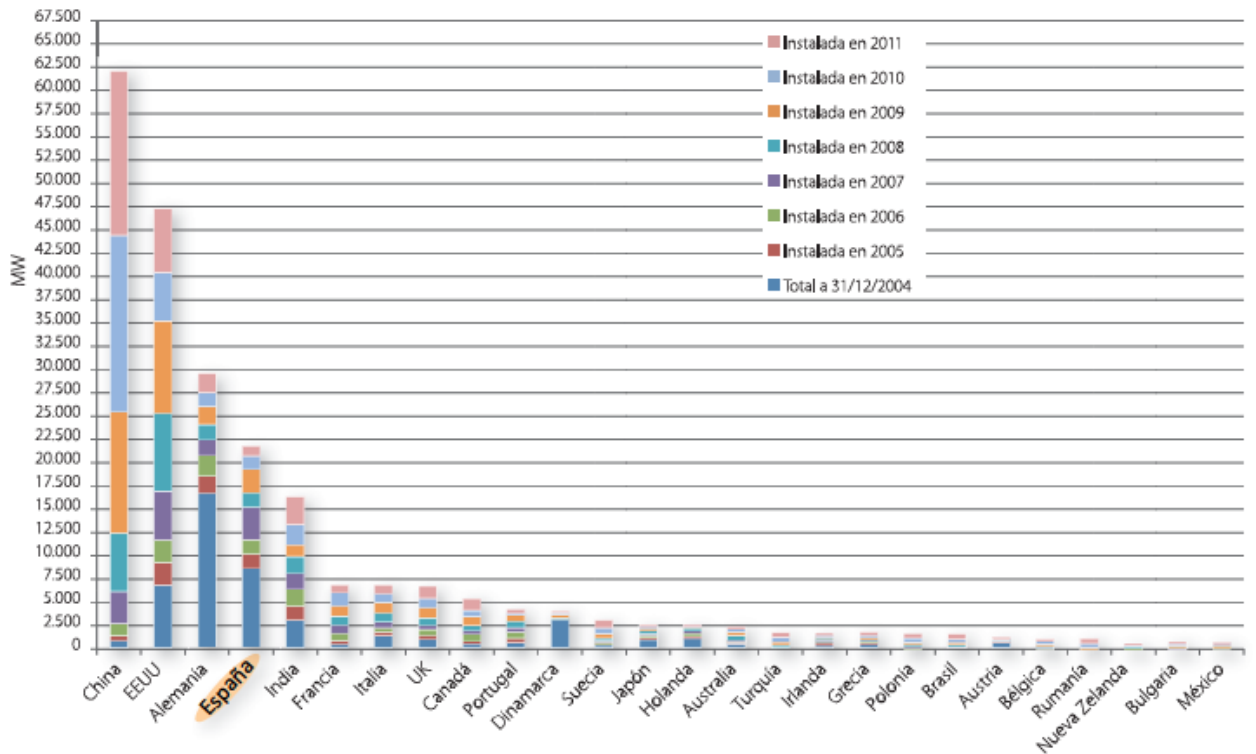

Figura 1.4: Evolución de la potencia eólica instalada anualmente en el mundo, AEE (2012). 


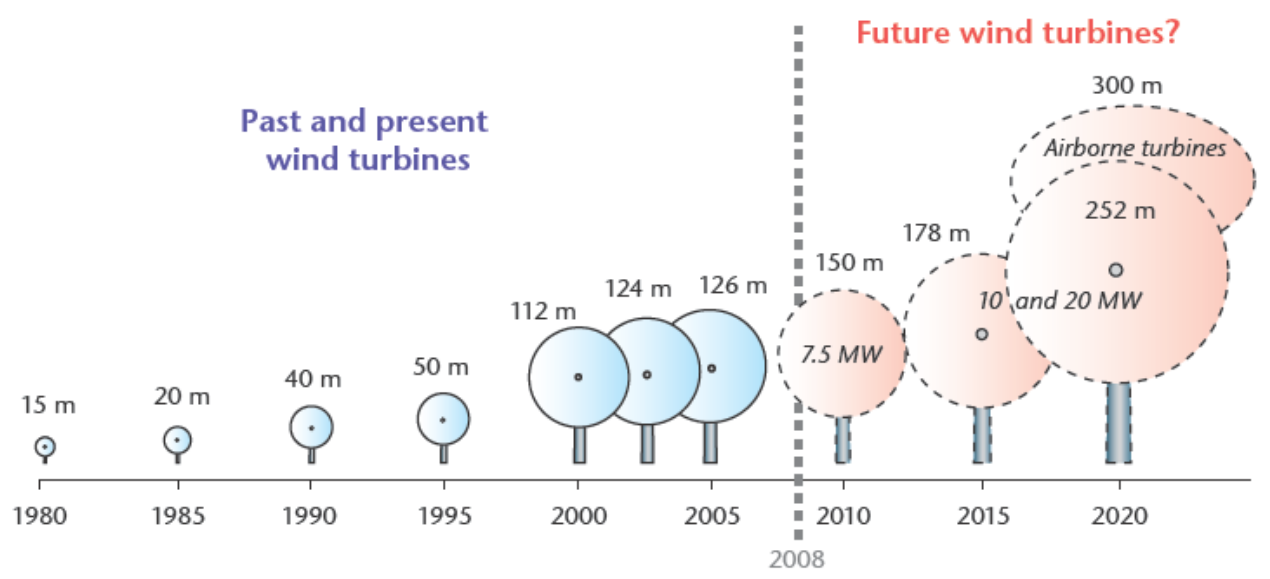

Figura 1.5: Evolución del tamaño de los aerogeneradores, EWEA 2009).

toda el área barrida del rotor, es decir, que la velocidad del viento no varía a lo largo de todo el diámetro del rotor eólico. Cuando se trata de aerogeneradores de pequeño tamaño, dicha suposición se puede acercar con cierto grado de exactitud a la realidad; pero en los aerogeneradores actuales dotados de grandes diámetros y alturas de buje conduce a errores significativos; ya que la velocidad del viento varía considerablemente a lo largo del área barrida por el rotor debido a diversos factores, tanto meteorológicos como topográficos, Honrubia et al. (2010d c b 2012); Courtney et al. (2008b); Antoniou et al. (2007a); Foussekis et al. (2007); Foussekis (2009); Gottschall et al. (2012). Además, es importante tener en cuenta la tendencia que existe orientada hacia el progresivo aumento del tamaño de los aerogeneradores, tal y como muestra la figura 1.5 EWEA (2009). En esta línea, uno de los mayores aerogeneradores comerciales que existen hoy en día pertenece al fabricante alemán Enercon y tiene una altura de buje de $135 \mathrm{~m}$ y un diámetro de rotor de $127 \mathrm{~m}$, Honrubia Escribano et al. (2012a); al igual que Siemens también tiene un prototipo muy avanzado de un aerogenerador de $154 \mathrm{~m}$ de diámetro de rotor.

La realización de mediciones a varias cotas sobre el área barrida del rotor se puede efectuar empleando diferentes técnicas. La más común es utilizar los sistemas de anemometría convencionales instalados en la torre meteorológica del parque eólico, tratándose en la mayoría de las ocasiones de anemómetros de cazoletas, anemómetros sónicos y anemómetros de hélice. El principal problema que aparece en este tipo de mediciones es el económico, dado que el coste de la torre meteorológica se incrementa proporcionalmente con la altura de la misma; por lo que para los grandes aerogeneradores que hoy en día se están instalando a nivel mundial, la rentabilidad de la construcción de una torre meteorológica a alturas superiores a la del buje resulta comprometida. 


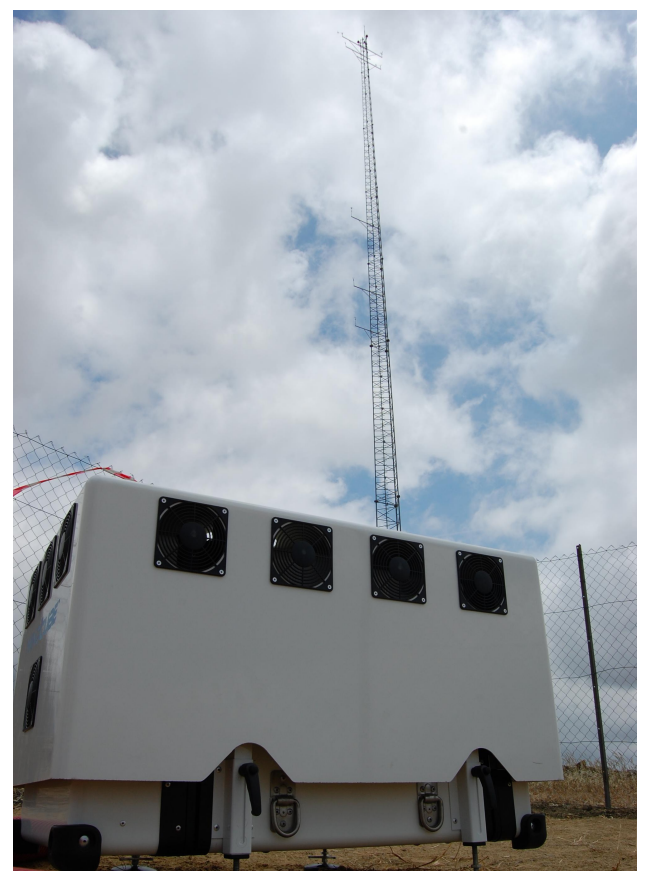

Figura 1.6: Equipo LiDAR (delante) junto a una torre meteorológica (detrás) en un parque eólico, Honrubia et al. (2010d).

Esta circunstancia representa una de las principales razones del empleo de tecnologías remotas para la medición del viento, más concretamente, tecnologías "Light Detection And Ranging (LiDAR)" o "Sound Detection And Ranging (SoDAR)"; ya que permiten la monitorización de la velocidad y dirección del viento sobre diferentes alturas definidas por el usuario. Otras ventajas destacables de estas recientes aplicaciones en el campo de la energía eólica son su rápida y fácil instalación, ya que pueden ubicarse en cualquier tipo de terreno en un tiempo aproximado de una hora; así como su mínimo impacto visual al poseer un volumen muy reducido y no necesitar de una torre auxiliar en la que alojar los diferentes anemómetros. En la figura 1.6 se muestra una primera imagen introductoria donde se observa un equipo LiDAR instalado junto a la torre meteorológica de un parque eólico del sur de España, Honrubia et al. (2010d).

Mientras que se puede considerar que existe cierto grado de madurez en sistemas convencionales de medición del recurso eólico, el desconocimiento de esta reciente aplicación tecnológica se hace patente en el sector eólico tanto a nivel nacional como internacional. La afirmación anterior está basada en artículos de investigación publicados tanto en congresos como en revistas especializadas durante los últimos años dentro de esta temática. Por ello, al tratarse la tecnología LiDAR de un sistema relativamente moderno pa- 
ra aplicaciones de energía eólica, los primeros artículos de investigación que aparecieron hace unos años estaban centrados en la evaluación de su comportamiento frente a sistemas convencionales, Smith et al. (2006); Courtney et al. (2008b); Foussekis (2009); Honrubia et al. (2010a, 2011). Posteriormente, una vez comprendidas las características de estos sistemas, la investigación se centró en estudiar la influencia del perfil de viento en la generación eólica, dado que estas tecnologías permiten la medida de parámetros eólicos a alturas superiores a las de una torre meteorológica, Wagner et al. (2009); Honrubia et al. (2010c b, 2012); Gottschall et al. (2012).

Las conclusiones de los estudios anteriores han tenido una considerable influencia sobre la Norma Internacional mencionada anteriormente, (International Electrotechnical Commission, 2005), que actualmente se encuentra en versión "Committee Draft (CD)", en la que todos los miembros de la misma son requeridos para alegar los comentarios oportunos y así construir la versión definitiva de la Norma. Es importante señalar que los directores de la Tesis Doctoral poseen contacto directo con miembros del Comité Técnico de dicha Norma, que se estima que se publique el próximo julio del año 2013. De hecho, es conocido que estas nuevas técnicas remotas serán incluidas como sistemas de medida capaces de caracterizar la curva de potencia de aerogeneradores, en lugar de realizarse exclusivamente con anemómetros convencionales tal y como señala la versión vigente de dicha Norma. Además, también incluirá aspectos relacionados con el perfil de viento, Albers et al. (2012).

En este punto es importante mencionar que, en el año 2008, una de las líneas de investigación señaladas como prioritarias por la Plataforma Tecnológica Europea de la Energía Eólica — conocida como "European Technology Platform for Wind Energy (TPWIND)"-, es el empleo de este tipo de tecnologías remotas para la medición del recurso eólico a alturas superiores a los $100 \mathrm{~m}$, TPWIND (2008).

Además de la contribución al desarrollo normativo anterior que, sin duda, contribuye a una óptima integración de la energía eólica en el sistema eléctrico, uno de los directores de la presente Tesis Doctoral es el responsable nacional del Comité Técnico de la Norma IEC 61400-27, (International Electrotechnical Commission, CD version) - actualmente sólo existen 43 miembros en todo el mundo, Sørensen et al. (2012) — , dedicada al desarrollo de modelos simplificados de aerogeneradores y parques eólicos. Debido al aumento progresivo de la potencia eólica instalada, resumido en las figuras 1.1 y 1.4 los operadores de las redes eléctricas necesitan modelos dinámicos que representen generación de energía eólica para poder realizar estudios de estabilidad de los sistemas eléctricos de potencia; con el objetivo de evaluar el impacto de la integración de este tipo de energía en los sistemas de energía eléctrica, Honrubia Escribano et al. (2012c).

La inmensa mayoría de los modelos desarrollados de generadores eólicos 
han sido realizados por los propios fabricantes. Esto ocasiona que, por un lado, los modelos sean específicos para cada tipo de aerogenerador y, por otro, que suelen estar firmados bajo contratos de confidencialidad, restringiendo el acceso a la información. Además, se trata de modelos detallados que, aunque permiten conocer con elevada precisión el comportamiento interno del aerogenerador, el tiempo de simulación también resulta incrementado. Por lo tanto, tal y como afirma la "North American Electric Reliability Corporation (NERC)", esta situación dificulta el análisis de la estabilidad de las redes eléctricas, NERC (2010). Este escenario puede llegar a ser insostenible conforme la energía eólica adquiera aún más importancia en el "mix energético", es decir, con estos modelos se persigue una integración de la energía eólica en las redes eléctricas. Por lo tanto, en este ámbito, resulta completamente necesario la implementación de modelos simplificados — también conocidos como genéricos o estándar- de aerogeneradores que, aunque su precisión no sea tan elevada como la de los modelos detallados, sí que solventan los problemas mencionados anteriormente, Asmine et al. (2011); Sørensen et al. (2011); Honrubia Escribano et al. (2012c).

En relación con esta temática, la TPWIND también ha indicado algunas líneas de investigación prioritarias. Concretamente, cita textualmente que son necesarios "modelos/herramientas de simulación para investigar sobre la estabilidad del sistema eléctrico europeo", TPWIND (2008).

De esta manera, la contribución a esta Norma de nueva creación, IEC 61400-27, para la integración de la energía eólica en los sistemas eléctricos resulta evidente, pues dicha normativa se encuentra actualmente también en versión CD. Concretamente el primer borrador para la Parte 1 se publicó en diciembre del año 2011 y se estima que el borrador definitivo, "Committee Draft for Voting (CDV)", se obtendrá a finales de 2012 en base a las contribuciones de cada Comité Nacional, tal y como se muestra en el cronograma de la figura 1.7, Sørensen (2012).

Los modelos de simulación planteados en la Norma International Electrotechnical Commission (CD version) están definidos para representar el comportamiento de la generación eólica en estudios de estabilidad de las redes eléctricas ante grandes perturbaciones en la tensión de suministro, como es el caso de los huecos de tensión. Dado que este tipo de fenómenos ocurren de manera aleatoria en las redes eléctricas debido a diferentes situaciones, Honrubia-Escribano et al. (2012b), la evaluación del comportamiento de la aparamenta eléctrica que se encuentra en los parques eólicos frente a dichas perturbaciones también resulta ser un tema de elevado interés dentro del sector eléctrico, Duran-Gomez et al. (1999); Bollen y Zhang (2000); Barros y Diego (2002); Pedra et al. (2005); Djokic et al. (2005b); Honrubia Escribano et al. (2010); Hannan et al. (2012); Honrubia-Escribano et al. (2012a). Además, mientras que ha quedado demostrado que el campo de investigación descrito en los párrafos anteriores incide de manera directa sobre aspectos re- 
Part 1

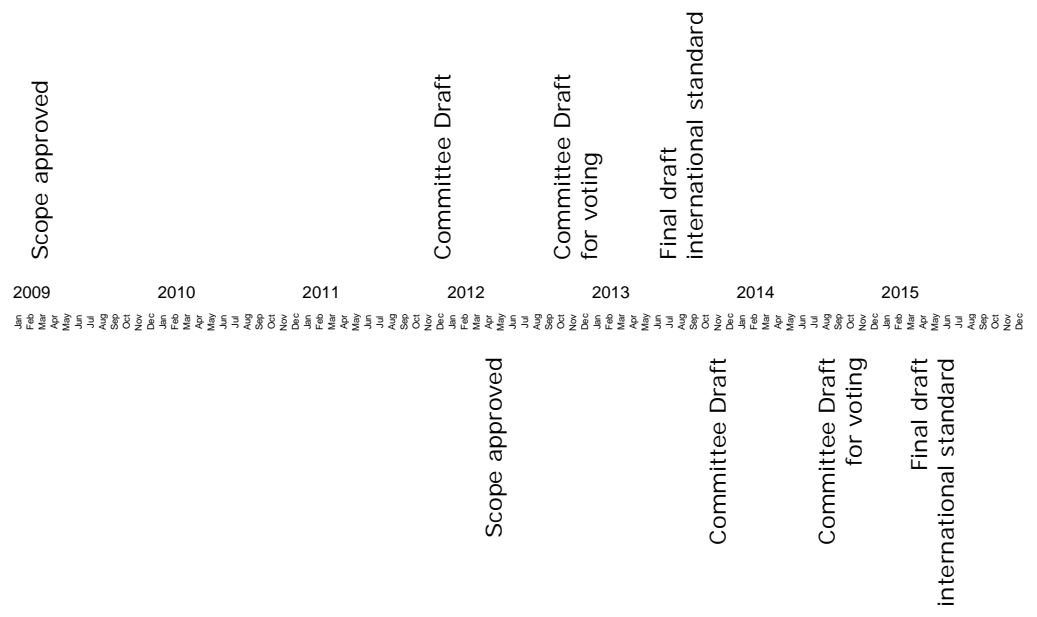

Figura 1.7: Cronograma estimado de desarrollo de la IEC 61400-27, Sørensen (2012).

gulatorios relacionados con la integración de la energía eólica en los sistemas de energía eléctrica, en este caso se puede considerar que existe un menor desarrollo normativo. Por lo tanto, la presente Tesis Doctoral también aporta relevantes resultados relacionados con el comportamiento de determinado equipamiento eléctrico, presente tanto en parques eólicos como en cualquier sistema de energía eléctrica, ante huecos de tensión e interrupciones del suministro.

Directamente vinculado con este ámbito tratado en la Tesis Doctoral, la TPWIND indica como línea de investigación prioritaria, dentro del marco de las estrategias de operación y mantenimiento de parques eólicos, la investigación de los efectos de las faltas en las redes eléctricas sobre los componentes de los aerogeneradores, TPWIND (2008).

Con todo lo anterior, se puede dar por concluido este primer apartado que resume de manera global cada uno de los temas que se analizarán en profundidad en capítulos posteriores. Por lo tanto, a continuación se presentan los objetivos perseguidos en la investigación y la estructura del documento. 


\subsection{Objetivos}

El objetivo principal de la presente Tesis Doctoral es la contribución a diferentes aspectos normativos de vital importancia para la adecuada integración de la energía eólica en las redes eléctricas.

Dado que se han considerado diversas cuestiones regulatorias, es necesario contemplar varios objetivos. En cuanto a la respuesta del equipamiento eléctrico frente a perturbaciones en las redes eléctricas, se persiguen los siguientes objetivos:

- Analizar las diferentes Normas que definen las curvas de tolerancia de equipos eléctricos.

- Caracterizar la respuesta de determinada aparamenta eléctrica bajo diferentes condiciones de carga ante huecos de tensión.

En cuanto a la caracterización de la curva de potencia de aerogeneradores, se pueden mencionar tres objetivos íntimamente relacionados que son abordados en la IEC 61400-12-1:

- Examinar las propiedades de los sistemas LiDAR y analizar su comportamiento frente a las tecnologías convencionales de medición del recurso eólico.

- Caracterización de la curva de potencia de aerogeneradores.

- Estudiar la influencia del gradiente de viento en la generación eólica.

Por último, en relación con el desarrollo de la Norma IEC 61400-27-1, se plantean los siguientes objetivos:

- Colaborar en la definición de especificaciones de modelos simplificados de aerogeneradores.

- Ajuste e implementación de modelos de determinados componentes del aerogenerador basado en datos reales recogidos en un parque eólico. 


\subsection{Estructura de la Tesis}

La presente Tesis Doctoral está dividida en cinco capítulos. Previo a esta organización se presenta un breve resumen de la temática investigada así como los índices del documento. Finalmente, se ha dispuesto una lista de referencias bibliográficas y una lista de acrónimos.

En este primer capítulo se ha presentado una introducción general al contexto actual de la energía eólica. De esta manera, se ha permitido mostrar las razones que han motivado la presente investigación, así como los objetivos perseguidos.

En el capítulo 2 se plantea de la problemática de la calidad de suministro en las redes eléctricas y, especialmente, de los huecos de tensión e interrupciones. Posteriormente, se analiza el comportamiento de diverso equipamiento eléctrico presente, tanto en centrales de generación eólica como en entornos industriales, frente a dichas perturbaciones de red. También, se realiza un estudio del carácter normativo en este ámbito.

En el capítulo 3 se exponen las aportaciones a la Norma IEC 61400-12-1. Para ello, se introducen algunas nociones teóricas del viento y se presenta un estado del arte sobre los métodos de medición del recurso eólico, con especial énfasis en la tecnología LiDAR, A continuación se presentan las propiedades de los diferentes parques eólicos donde se han efectuado las campañas de medida. Para finalizar, se abordan dos temas de elevado interés tecnológico dentro del sector eólico. Por una parte, se presenta un estudio comparativo entre mediciones efectuadas con el equipo LiDAR y con anemómetros convencionales y, finalmente, se muestra la influencia del gradiente de viento en la generación eólica.

El capítulo 4 resume, de manera cronológica, el trabajo desarrollado hasta la fecha por el Comité Técnico encargado del desarrollo de la Norma IEC 61400-27. Por lo tanto, se detallan las especificaciones de los modelos simplificados de aerogeneradores. Además, se han implementado algunos modelos de determinados componentes del aerogenerador; así como de elementos de control del mismo.

Para finalizar, el capítulo 5 contiene las conclusiones derivadas de la presente Tesis Doctoral. Además, recoge las aportaciones novedosas que han surgido objeto de la investigación y plantea diversas alternativas por las que dar continuidad al trabajo desarrollado. 

Capítulo 2

\title{
Estudio de la respuesta de diversa aparamenta eléctrica ante huecos de tensión
}

\begin{abstract}
Resumen: En este capítulo se analiza en profundidad el comportamiento de determinada aparamenta eléctrica presente en centrales de generación de energía eólica ante determinadas perturbaciones existentes en los sistemas de energía eléctrica. En un primer apartado se introduce la temática de la calidad de suministro así como de las perturbaciones en las redes eléctricas. Posteriormente se presenta el estado del arte en este ámbito. Más adelante se detalla la metodología seguida en el transcurso de los ensayos y, finalmente, se muestran los resultados y conclusiones sobre la aparamenta analizada.
\end{abstract}

\subsection{Calidad del suministro eléctrico}

La calidad del suministro eléctrico ha sido y sigue siendo una preocupación importante para las empresas del sector eléctrico, Gencer et al. (2010); Vegunta y Milanovic (2011). Sin embargo, no es fácil definir lo que debe entenderse como una calidad de la energía eléctrica aceptable, dado que la que puede resultar idónea para un tipo de carga es posible que no sea aceptable para otro tipo de cargas más sensibles.

Por este motivo, resulta complejo encontrar una definición única de calidad de suministro. Los puntos de vista de la empresa suministradora, del fabricante de equipos y de los clientes son completamente diferentes. Esencialmente, la posición de la compañía suministradora se basa en el cumplimiento de determinadas normas que, aunque se analizarán en detalle más 
adelante, se pueden encontrar relacionadas con el valor eficaz de la tensión o con el contenido de armónicos, por ejemplo. Por otro lado, los fabricantes la perciben como aquel nivel de suministro que permite el correcto funcionamiento de sus equipos; mientras que los clientes consideran una buena calidad de suministro la que garantiza que sus procesos, operaciones y/o negocios no se vean afectados, Brown (2009).

Básicamente, la calidad del suministro eléctrico es la interacción de la energía eléctrica con los distintos equipos eléctricos. Si los equipos eléctricos operan de manera correcta, sin ser dañados o perjudicados, se puede decir que el suministro eléctrico es de calidad, Dwivedi et al. (2008). Por el contrario, si el equipo eléctrico no opera adecuadamente entonces la calidad del suministro eléctrico es insuficiente.

Cuando la calidad del suministro eléctrico disponible no es suficiente para satisfacer las necesidades del usuario, es necesario aplicar medidas de mejora de la misma. Como es de esperar, estas medidas de mejora están ligadas con un coste que muchos autores han tratado de cuantificar, Vegunta y Milanovic (2011). Por ejemplo, para una industria dedicada al sector de la fabricación, la solución a problemas derivados con la calidad de suministro puede oscilar entre $388 €$ y $1165 €$ por $\mathrm{kW}$ instalado, Stephens (2007). Por el contrario, ser vulnerable a estas perturbaciones ocasiona unas pérdidas relevantes. Concretamente, McGranaghan y Roettger (2002) estiman que las pérdidas causadas por la mala calidad de la energía generan un coste anual cercano a 10.000 millones de dólares a la industria debido a interrupciones en el proceso industrial. Otro ejemplo lo constituyen las grandes empresas del sector de fabricación de semiconductores, llegando a perder hasta $830.000 €$ por cada hueco de tensión registrado en sus instalaciones, Cornick y Li (2000).

Hay que tener en cuenta que la eliminación por completo de las perturbaciones electromagnéticas es imposible. Por ello, los equipos se han de preparar para disminuir el impacto de las mismas sobre ellos. Esta circunstancia da origen a un debate: por una parte, los consumidores señalan que ellos no son los responsables de la generación de los huecos de tensión; mientras que las compañías suministradoras de energía eléctrica mantienen el argumento de que los equipos de los consumidores son extremadamente sensibles y que la red no puede diseñarse para que no exista falta alguna en ella. A modo de ejemplo, en Bollen (2000) se menciona que existen equipos que fallan al producirse una disminución de la tensión de alimentación un $10 \%$ durante 1 ó 2 ciclos (en el apartado 2.2 se amplía considerablemente esta información).

En relación con la reglamentación internacional en este ámbito, el "Institute of Electrical and Electronics Engineers (IEEE)" posee una Norma desarrollada, IEEE Std. 1159-2009, donde se define la calidad de suministro como "el concepto de alimentación y puesta a tierra de un equipo electrónico de forma que sean adecuados para la operación de dicho equipo y compatibles con el sistema de cableado existente y el funcionamiento de otros equipos 
conectados".

De entre todas las perturbaciones electromagnéticas que pueden afectar a la calidad de suministro, las tres más importantes desde el punto de vista del consumidor son las siguientes:

- Huecos de tensión.

- Interrupciones momentáneas.

- Interrupciones prolongadas.

Como se señaló anteriormente, cada tipo de consumidor conectado a la red eléctrica se comportará de diferente manera frente a una perturbación de red. A la mayoría de los clientes residenciales les afectan en mayor grado las interrupciones, ya sean momentáneas o prolongadas; mientras que para clientes industriales, los huecos de tensión y las interrupciones momentáneas son los problemas más comunes a los que se enfrentan.

También, hay que señalar que, lógicamente, un hueco de tensión no es tan perjudicial como lo sería una interrupción para una industria, pero debido a que la ocurrencia de los huecos es mucho mayor que la de las interrupciones, el impacto total debido a huecos debe ser tenido en consideración, Sarmiento y Estrada (1996); Won et al. (2003); Moreno-Munoz y De la Rosa (2008); Deswal et al. (2009); Flores-Arias et al. (2011); Honrubia-Escribano et al. (2012b). De hecho, hay estadísticas que indican que en países desarrollados la relación entre huecos de tensión e interrupciones es del orden de 10:1, e incluso en la isla de Singapur llega a ser de 50:1, Cornick y Li (2000).

Según IEEE Std. 1159-2009, los huecos de tensión así como las breves interrupciones se encuadran dentro de las variaciones de valor eficaz de corta duración. Cada tipo de perturbación se puede definir como instantánea, momentánea, o temporal, en función de su duración. A modo de resumen, en la tabla 2.1. se presentan todas las perturbaciones de corta duración recogidas por dicha normativa. Este tipo de perturbaciones de corta duración son producidas en la mayoría de los casos por faltas en las redes eléctricas o por la conexión de grandes cargas. En función de la localización exacta de la falta y de las características del sistema eléctrico en que se encuentre, la falta podrá ocasionar sobretensiones, huecos de tensión o una completa pérdida de tensión denominada como interrupción. A modo de recopilatorio, el IEEE recientemente ha publicado una guía en la que describe en detalle los fenómenos relacionados con la calidad del suministro eléctrico, IEEE Std. 1250-2011,

Dada la importancia de este tipo de perturbaciones, a continuación se describen en mayor profundidad. 


\begin{tabular}{l|c|c}
\hline Tipo de perturbación & Duración & Magnitud de tensión \\
\hline Instantánea & & \\
Hueco & $0.5-30$ ciclos & $0.1-0.9 \mathrm{pu}$ \\
Sobretensión & $0.5-30$ ciclos & $1.1-1.8 \mathrm{pu}$ \\
\hline Momentánea & $0.5-3 \mathrm{~s}$ & $<0.1 \mathrm{pu}$ \\
Interrupción & 30 ciclos-3 s & $0.1-0.9 \mathrm{pu}$ \\
Hueco & 30 ciclos-3 s & $1.1-1.4 \mathrm{pu}$ \\
Sobretensión & & \\
\hline Temporal & $3 \mathrm{~s}-1 \mathrm{~min}$ & $<0.1 \mathrm{pu}$ \\
Interrupción & $3 \mathrm{~s}-1 \mathrm{~min}$ & $0.1-0.9 \mathrm{pu}$ \\
Hueco & $3 \mathrm{~s}-1 \mathrm{~min}$ & $1.1-1.2 \mathrm{pu}$ \\
Sobretensión &
\end{tabular}

Tabla 2.1: Tipos de perturbaciones de tensión de corta duración según IEEE Std. 1159-2009.

\subsubsection{Huecos de tensión e interrupciones}

Los huecos de tensión representan uno de los problemas de calidad de suministro más comunes y, al mismo tiempo, más difíciles de resolver. Concretamente, se puede afirmar que el $80 \%$ de las quejas de los consumidores con relación a la calidad de suministro son debidas a los huecos de tensión, Akolkar y Kushare (2010).

Tal y como se puede observar en la tabla 2.1, IEEE Std. 1159-2009, un hueco de tensión se define como un descenso en el valor eficaz de la tensión situado entre 0.1 pu y 0.9 pu, y con una duración comprendida entre 0.5 ciclos y 1 min.

En la figura 2.1 se han mostrado los parámetros que definen un hueco de tensión. El parámetro $V_{t r}$ ("Voltage threshold") representa el límite de tensión por debajo del cual se considera iniciado el hueco de tensión $(0.9 \mathrm{pu}$ según IEEE Std. 1159-2009). La profundidad del hueco es la diferencia entre la tensión nominal y la residual - ésta también se conoce como magnitud de tensión- . Tal y como se ha señalado en dicha figura, la duración del hueco es el tiempo transcurrido desde el instante en que se produce el hueco (la tensión cae por debajo de $V_{t r}$ ) hasta que la misma se eleva por encima de dicho valor.

En el caso de que un hueco de tensión afecte a más de una de las fases de un sistema eléctrico, normalmente se considera como tensión residual la más reducida de las tres fases, y la duración es el tiempo total en que la tensión eficaz en una de las fases siga siendo inferior a $0.9 \mathrm{pu}$, tal y como se indica en la Norma IEC 61000-4-30, International Electrotechnical Commission (2008). 


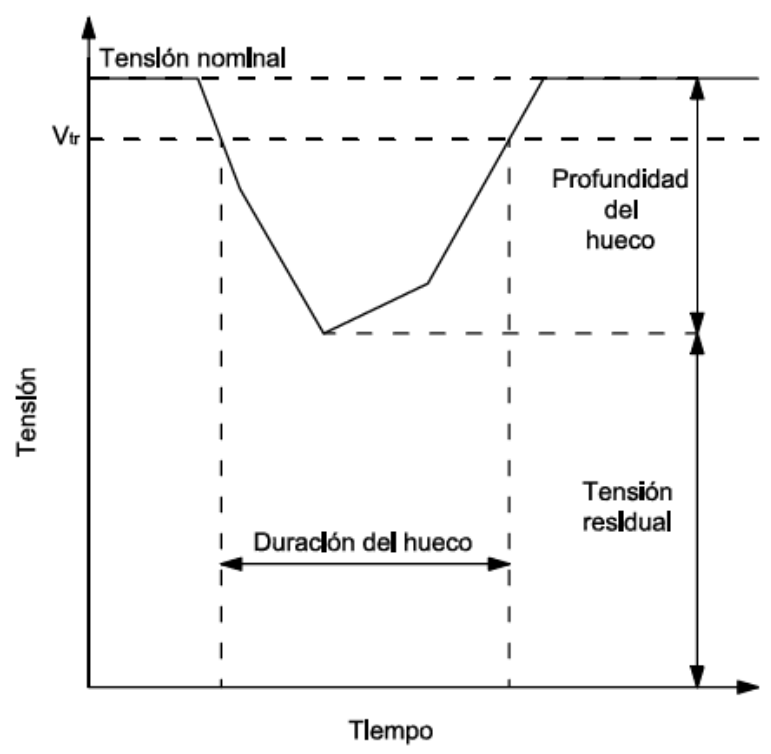

Figura 2.1: Definición de los parámetros de un hueco de tensión.

La desventaja principal que plantea este método es que un hueco de tensión en una fase se caracterizaría igual que un hueco trifásico, mientras que los huecos trifásicos suelen ser más severos para las cargas que alimentan, Bollen y Zhang 2003.

Los huecos de tensión pueden tener un origen muy diverso, por ejemplo, faltas en las redes eléctricas, conexión de grandes cargas, conexión de grandes motores, etc. Para resumir las principales causas de de los huecos de tensión, la figura 2.2 muestra diversos tipos de huecos típicos producidos por diferentes circunstancias. El número indicado sobre cada región de dicha figura se encuentra asociado con el siguiente listado de causas comunes de huecos de tensión:

1. Faltas en la red de transporte: este tipo de faltas puede producir huecos de tensión de profundidad variable: desde pequeña profundidad hasta más del $50 \%$ de la misma. La duración de este tipo de huecos es bastante reducida, oscilando entre $50 \mathrm{~ms}$ y $100 \mathrm{~ms}$.

2. Faltas en redes de distribución remotas (alejadas de la carga): este tipo de faltas origina huecos de poca profundidad debido a la impedancia del transformador. Su duración será como máximo de unos segundos.

3. Faltas en la red de distribución local (cercanas a la carga): debido a que la carga se sitúa en un lugar más próximo a donde se origina la falta, la forma del hueco será similar al caso anterior, pero con mayor profundidad y ligeramente mayor duración. 


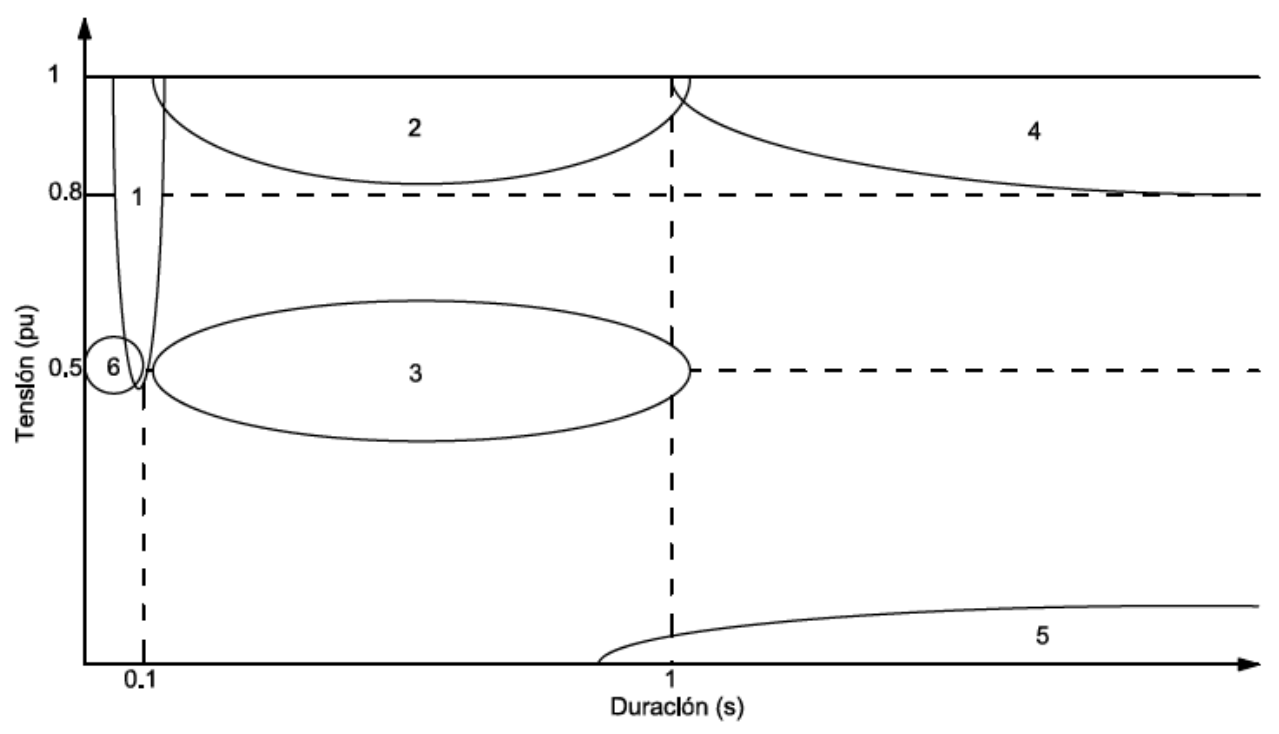

Figura 2.2: Diferentes tipologías de huecos de tensión.

4. Arranques de grandes motores: este tipo de maniobra generará huecos de poca profundidad; aunque su duración se puede prolongar considerablemente.

5. Interrupciones: (comentadas más adelante).

6. Actuaciones de fusibles e interruptores automáticos: este tipo de maniobras pueden producir huecos de elevada profundidad si la falta se produce en la red de distribución local; aunque suelen ser de duración reducida, Brauner y Hennerbichler (2001).

Una vez resumido de una manera muy breve qué son los huecos de tensión, se tratan a continuación algunos aspectos relacionados con las interrupciones del suministro que ocurren en los sistemas eléctricos de potencia. Como se indicó en la tabla 2.1 una interrupción ocurre cuando la tensión de alimentación se reduce a menos de 0.1 pu durante un período de tiempo inferior a $1 \mathrm{~min}$. En el caso de las interrupciones no es necesario definir una serie de parámetros que las caractericen; ya que se interpretan en función de su duración dado que la tensión se encontrará por debajo del $10 \%$ de la nominal en cualquier caso. La diferencia fundamental entre una interrupción momentánea o prolongada estriba en la duración, siendo tres segundos el límite característico.

En algunos casos, cuando la interrupción se debe a una falta en la red, las interrupciones suceden precisamente después de un hueco de tensión. El hueco de tensión ocurre dentro del intervalo en que se produce la falta y el 
elemento de protección de la línea, subestación, etc., actúa. De esta manera, una carga podrá experimentar primero un hueco de tensión y, posteriormente, una interrupción, Moon et al. (2007). La duración de la interrupción dependerá de la capacidad de apertura-cierre ("reclosing") del dispositivo de protección, que puede situarse dentro del intervalo comprendido entre $0.2 \mathrm{y}$ 2 segundos, Makinen et al. (2011).

\subsubsection{Curvas de tolerancia}

La respuesta de un equipo cuando resulta sometido a una perturbación en la red eléctrica es un tema debatido tanto por los fabricantes de equipos como por los usuarios de los mismos; así como por los operadores del sistema eléctrico. Generalmente, para representar el comportamiento de un equipo ante un hueco de tensión, suelen construirse gráficos en dos dimensiones que indican en el eje vertical el nivel de tensión, y en el horizontal el tiempo durante el que se aplica dicha tensión, Dorr et al. (1997b); Short (2004). Este tipo de curvas se conocen como curvas de tolerancia, o "Power Acceptability Curves", Kyei et al. (2002), o "Voltage Tolerance Curves", Bollen et al. (2010). Una vez que se posee esta curva característica, algunos autores la han empleado para evaluar, desde un punto de vista económico dónde resulta interesante realizar cambios, por ejemplo, si una modificación de las redes de suministro resultará más rentable que una modificación de la capacidad de soportar huecos de tensión de los equipos, Kushare y Ghatol (2007).

Como se indicó anteriormente, este tipo de curvas representan la variación de la tensión de alimentación - normalmente expresado en tanto por ciento- frente al tiempo - expresado en segundos o ciclos-. Constituyen la forma más recomendable de representar el comportamiento de un equipo frente a huecos de tensión en particular, y frente a variaciones de tensión en el suministro de la energía eléctrica en general. En dichas curvas, un hueco se define simplemente por su profundidad o por su magnitud y su duración (ver parámetros definidos en la figura 2.1). Se asume igualmente que el perfil de tensión de todo hueco es rectangular. Esta suposición sería válida en algunas circunstancias ideales; pero hay que tener en cuenta que existen huecos de tensión que poseen una recuperación a fallos mucho más prolongada, alejándose, por tanto, la forma rectangular ideal.

Los huecos de tensión de tipo no rectangular se dan en algunos sistemas de energía industriales con grandes cargas de motores de inducción. Esto se debe a que, cuando la tensión se reduce en los terminales del motor, el par y la velocidad del motor son proporcionalmente reducidos. Posteriormente, cuando el hueco ha desaparecido y, por tanto, ha aumentado la tensión hasta su valor nominal, la máquina eléctrica absorbe de la red una elevada intensidad para crear de nuevo el campo magnético en el entrehierro y así volver a situar al motor en condiciones nominales de trabajo. Esta elevada necesidad de intensidad hacia el motor puede causar una falta posterior al hueco de 
duración entre un segundo o más, aproximadamente, tal y como se señaló en la figura 2.2. De esta manera, el hueco de tensión sufrido por el motor de inducción no es rectangular, sino que inicialmente tiene una caída muy fuerte $y$, al final, tiene una larga recuperación.

En el caso de los contactores, donde la presente Tesis Doctoral realiza un extenso estudio, Bollen (2000) menciona que los valores límites para la tensión en la curva de tolerancia puede variar entre el $40 \%$ y el $80 \%$ de la tensión nominal; mientras que el tiempo que pueden soportar $0 \%$ de tensión rondaría entre 0.5 ciclos y $4-5$ ciclos. No obstante, esta información es ampliamente detallada en el apartado 2.2.1. En este contexto, es importante señalar que existe una Norma donde se mencionan los límites de funcionamiento de los contactores, UNE-EN 60947-4-1; aunque lo único que indica es que estos equipos deben cerrar sus contactos de manera satisfactoria en cualquier valor entre el $85 \%$ y el $110 \%$ de su tensión nominal del circuito de control (ver definiciones en apartado 2.2.1).

En cambio, en UNE-EN 61000-4-11 se especifican diferentes tipos de ensayos para simular los efectos de las variaciones bruscas de tensión. Sin embargo, aún tratándose de la Norma vigente dedicada al ensayo de equipos, indica que los ensayos deberán realizarse solamente bajo determinadas tensiones residuales. Además, en cuanto al valor de inicio de la onda de tensión, señala que los ensayos deben realizarse al paso por cero de la tensión y en otros ángulos considerados como críticos (preferentemente $45^{\circ}, 90^{\circ}, 135^{\circ}$, $180^{\circ}, 225^{\circ}, 270^{\circ}$ y $315^{\circ}$ ). Posteriormente, en los apartados 2.2 y 2.4 se estudia la influencia del inicio de la onda de tensión sobre la respuesta de la aparamenta eléctrica. Particularmente para las cargas de iluminación conectadas a baja tensión existe una Norma específica, UNE-EN 61547, aunque sólo indica dos valores de ensayo ante huecos de tensión e interrupciones.

Desde el punto de vista del fabricante de equipos, la Normativa más importante sobre curvas de tolerancia ha sido desarrollada por el "Information Technology Industry Council (ITI)", figura 2.3. Esta curva describe la forma de la tensión alterna de alimentación que normalmente será tolerada por la mayoría de los equipos relacionados con las tecnologías de la información (ordenadores, fotocopiadoras, etc.), estando referida a redes de tensión nominal de $120 \mathrm{~V}$ y $60 \mathrm{~Hz}$. La curva ITI es la actualización de la antigua curva "Computer Business Equipment Manufacturer's Associattion (CBEMA)", debido a que, tal y como se apunta en Djokic et al. (2005a), la curva CBEMA es más complicada de reproducir y, por lo tanto, aumenta la complejidad de realización de los ensayos. Una primera observación sobre la curva ITI es que los equipos son más sensibles a las sobretensiones que a los huecos de tensión, Saied (2007).

Como puede verse en la figura 2.3, la curva ITI define tres zonas de funcionamiento de los equipos claramente diferenciadas:

- Región de funcionamiento sin interrupción: en esta zona, señalada con 


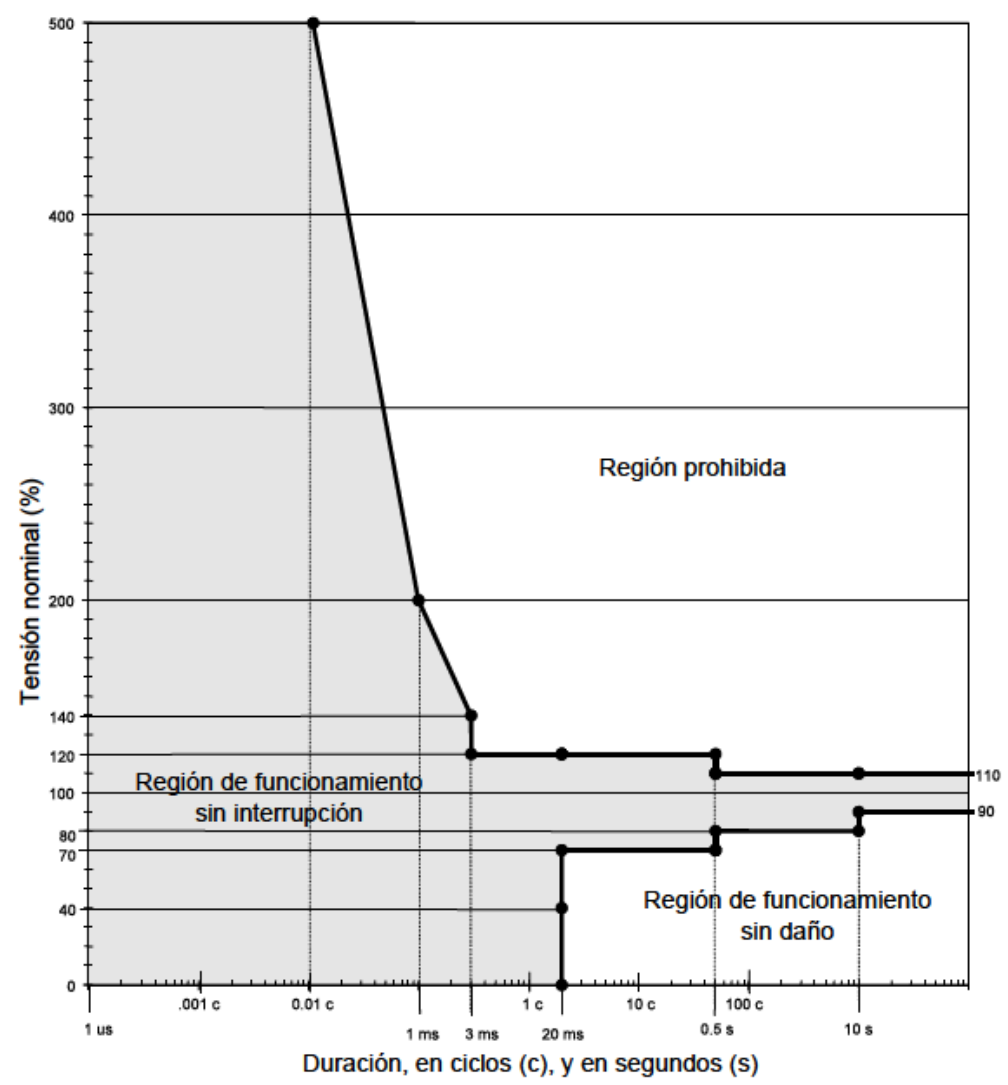

Figura 2.3: Curva ITI.

fondo gris sobre la figura 2.3. el equipo soporta cualquier variación de tensión dentro de los límites impuestos de tal manera que no se produzca ningún fallo en su funcionamiento ni en sus componentes.

- Región de funcionamiento sin daño: en esta zona se producirá una desconexión o un malfuncionamiento del equipo ensayado, sin sufrir sus componentes daño alguno.

- Región prohibida: en esta ocasión, el equipo expuesto podrá sufrir daños irreparables si la tensión se sitúa en dicha región.

Es importante señalar que la curva ITI no es una Normativa propiamente dicha, es decir, los equipos no tienen obligación de obtener la certificación de que cumplen dicha curva, pero sí que se ha utilizado como punto de referencia a la hora de evaluar la capacidad de variación de la tensión de alimentación de los equipos.

Además de la curva ITI mostrada anteriormente, existe otra curva de tolerancia creada hace unos años por la industria de los semiconductores, 


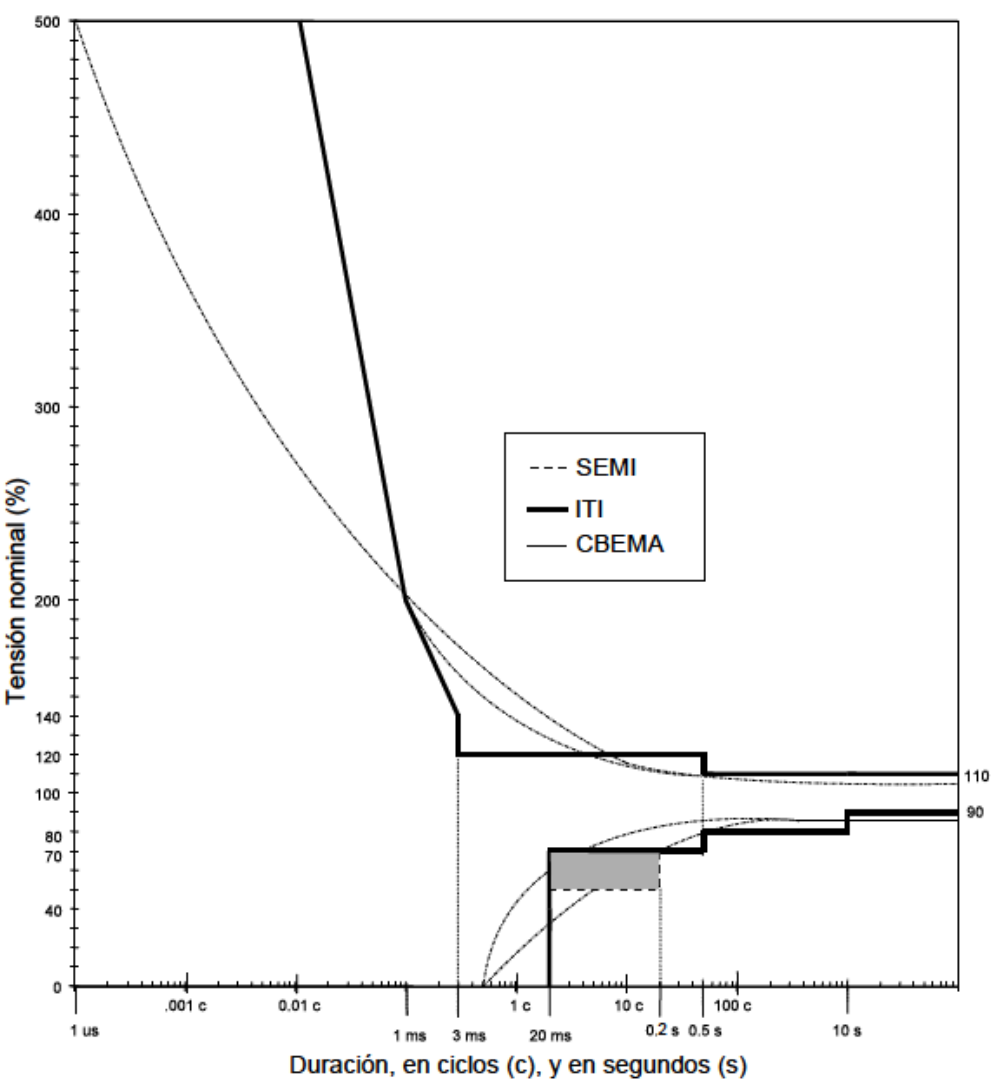

Figura 2.4: Comparativa de las curvas de tolerancia.

la SEMI F47-0706. La curva SEMI se centra exclusivamente en huecos de tensión; mientras que la ITI contempla también las sobretensiones. Concretamente, la curva SEMI describe tres escalones de tensión:

- Tensión residual del $50 \%$ : los equipos deben soportar este valor 10 ciclos (a $50 \mathrm{~Hz}$ ) ó 12 ciclos (a $60 \mathrm{~Hz}$ ).

- Tensión residual del $70 \%$ : los equipos deben soportar este valor 25 ciclos (a $50 \mathrm{~Hz})$ ó 30 ciclos (a $60 \mathrm{~Hz}$ ).

- Tensión residual del $80 \%$ : los equipos deben soportar este valor 50 ciclos (a $50 \mathrm{~Hz})$ ó 60 ciclos $($ a $60 \mathrm{~Hz})$.

Además de los tres puntos definidos anteriormente, la curva SEMI recomienda que los equipos soporten el $0 \%$ de la tensión nominal durante 1 ciclo, así como una tensión residual del $80 \%$ durante 500 ciclos (a $50 \mathrm{~Hz}$ ) ó 600 ciclos (a $60 \mathrm{~Hz}$ ), Chuck (2006). 
Resulta interesante comparar las diversas curvas de tolerancia. Por ello, en la figura 2.4 se muestran los tres tipos de curvas de tolerancia citados anteriormente. Sobre dicha figura, en color gris se observa que la curva SEMI es más estricta que la ITI en el intervalo entre $20 \mathrm{~ms}$ y $200 \mathrm{~ms}$, debido a la mayor profundidad de tensión que define (50\% frente a $30 \%$ ); aunque según la curva ITI, los equipos deben soportar un $0 \%$ de la tensión durante los primeros $20 \mathrm{~ms}$. Sin embargo, se puede afirmar, Short (2004), que algunos equipos superarán la curva SEMI, pero no la ITI. Un claro ejemplo de este tipo de equipos, son los relés y contactores, debido a que la ITI tiene una interrupción de 20 ms que puede ser suficiente para desconectar relés y contactores, los cuales sí que pueden soportar un hueco de $50 \%$ de profundidad durante $200 \mathrm{~ms}$ (el punto más crítico de la curva SEMI). En el apartado 2.2 se amplía esta información en base al trabajo realizado por otros autores y en el apartado 2.4 en función de los ensayos experimentales realizados en la presente Tesis Doctoral.

Como se ha visto, la curva de tolerancia de un equipo asume que una variación de tensión, ya sea un hueco o una sobretensión, se puede caracterizar únicamente por medio de su magnitud y de su duración. De esta manera, desde el punto de vista del equipo en estudio, si dos huecos de tensión (o dos sobretensiones) tienen la misma magnitud y la misma duración, ambos producirán el mismo efecto sobre el equipo, esto es, o se produce la desconexión del equipo en ambos casos o esta desconexión no se produce en ningún caso. Este supuesto puede resultar erróneo en determinada aparamenta eléctrica, como se verá más adelante, ya que el ángulo de fase inicial (éste no se encuentra reflejado en las curvas) hace variar significativamente el comportamiento del equipo frente a huecos de tensión. 


\subsection{Análisis del equipamiento ensayado}

En el apartado anterior se ha tratado la problemática de la calidad de suministro e introducido la terminología relativa a las curvas de tolerancia de los equipos. Continuando en la misma línea, el presente apartado muestra un estado del arte sobre los equipos que, además de encontrarse entre los más sensibles ante huecos de tensión, son comúnmente empleados en centrales de generación de energía eólica, siendo:

- Contactor.

- Relé de falta a tierra.

- Variador de frecuencia.

- Ordenador personal.

- Diversas cargas de iluminación.

Además de la revisión bibliográfica, en este apartado se resume el funcionamiento de cada uno de los equipos listados anteriormente.

Tal y como se podrá comprobar posteriormente en base al desarrollo experimental llevado a cabo en la presente Tesis Doctoral, apartado 2.4, la respuesta de cada uno de los equipos anteriores frente a huecos de tensión es completamente diferente. Como primer concepto, de manera general, se puede afirmar que, a excepción del contactor, el resto de aparamenta eléctrica analizada describe una curva de tolerancia prácticamente rectangular.

\subsubsection{Análisis del Contactor}

Los elementos de conmutación, entre los cuales se encuentran los contactores, son esenciales para el control, protección y aislamiento eficaz de cualquier sistema eléctrico. Son uno de los elementos eléctricos más ampliamente utilizados en instalaciones industriales, Riba et al. (2011), así como en parques eólicos, Wong et al. (2009), ya que pueden ser integrados junto a otro tipo de aparamenta y, todo el conjunto, realizar acciones más complejas tales como protección coordinada, automatización industrial, centralización o control remoto, etc. En esencia, un contactor es un conmutador controlado de manera eléctrica, que emplea una bobina para provocar el cierre de uno o más pares de contactos eléctricos cuando una determinada tensión se aplica sobre la bobina, Collins y Bridgwood (1997).

Los contactores están constituidos por:

- Carcasa o base: es el soporte en el que se fijan todos los componentes del contactor. Suele tener dos partes, la superior y la inferior. Se encuentra fabricado en material no conductor, con alto grado de rigidez y resistencia al calor. 
- Electroimán: es el elemento principal del contactor. Su función es transformar la energía eléctrica que recibe de la red en energía magnética; ya que ésta provocará el desplazamiento de la parte móvil del contactor (armadura). Está constituido por los siguientes tres componentes, siendo el más importante la bobina.

- Bobina: la intensidad que absorbe la bobina es la causante de la magnetización; y con ello se consigue el desplazamiento de la armadura sobre el núcleo. En función del tipo de contactor, la bobina puede alimentarse con corriente alterna o continua. Las que se alimentan con corriente continua generan un campo magnético constante que garantiza el cierre de los contactos. Sin embargo, las alimentadas con corriente alterna generan campos magnéticos senoidales que generan una fuerza magnética que se hace cero dos veces por ciclo, lo que podría ocasionar rebotes de la armadura. Para evitar este fenómeno, se les añade una espira de cortocircuito - también llamada espira de sombra- (conocido en la bibliografía como "shading ring") en los extremos del núcleo. De esta manera, la intensidad que fluye por esta espira genera un campo magnético en desfase con el creado por la bobina; con lo que se consigue que el campo magnético total no se anule en cada ciclo.

- Núcleo (también conocido como culata o yugo): parte metálica, de material ferromagnético que, dependiendo del tipo de contactor, es común que adopte la forma la letra "E", y se encuentra fijado a la carcasa. En su parte central suele contener a la bobina. Su función consiste en concentrar y aumentar el flujo magnético generado por la bobina.

- Armadura (algunos fabricantes la denominan martillo, Poza Alonso (2005)): es el contacto móvil. En cuanto a su morfología, es similar a la del núcleo pero sin la bobina ni las espiras de cortocircuito. En su parte posterior se encuentra unida a unos resortes con el objetivo de mantener los contactos principales del contactor abiertos cuando no exista campo magnético.

- Contactos: son los elementos conductores que van a permitir el flujo de intensidad en el momento en que se magnetice la bobina. Existen tanto contactos fijos unidos a la carcasa superior del contactor, como contactos móviles fijados a la armadura; permitiendo mediante la magnetización de la bobina que los contactos móviles se cierren sobre los fijos. En el contactor se encuentran dos tipos de contactos. Por un lado, los contactos principales — normalmente abiertos en reposo- están destinados para abrir y cerrar el circuito de potencia, permitiendo el paso de la intensidad nominal. Mientras que los contactos auxiliares - en reposo, pueden ser tanto normalmente abiertos como cerrados- 


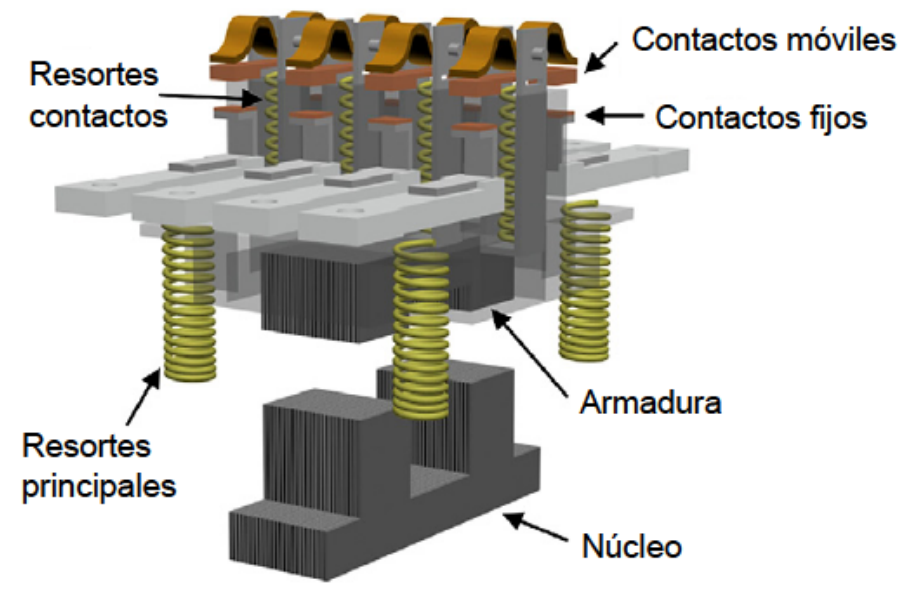

Figura 2.5: Imagen 3D de un contactor de corriente continua, Riba et al. (2011).

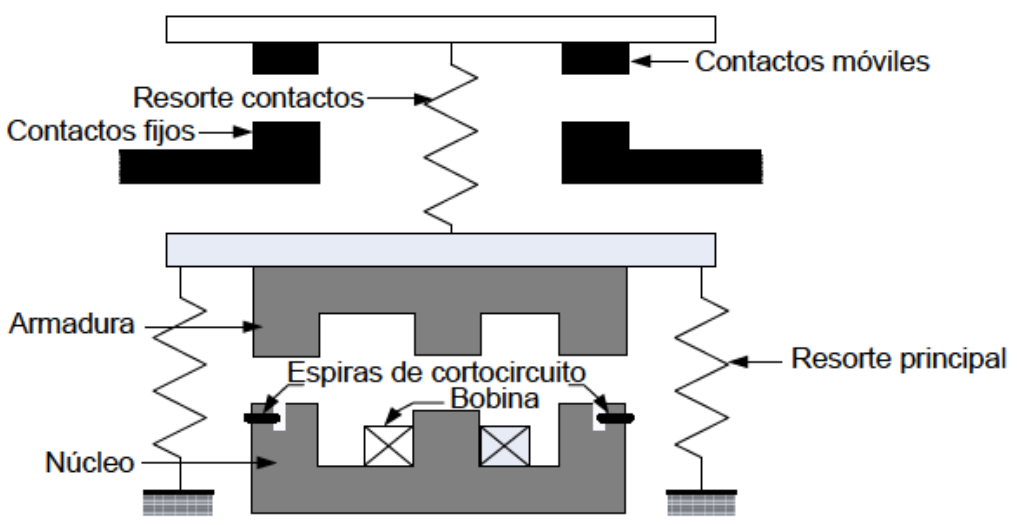

Figura 2.6: Esquema de un contactor de corriente alterna.

se encuentran acoplados mecánicamente a los contactos principales, encargándose de abrir y cerrar los circuitos auxiliares y de mando del contactor.

Con el objeto de ilustrar los conceptos anteriores y comprender más fácilmente los que se presentarán a continuación, las figuras 2.5 y 2.6 muestran dos esquemas de diferentes tipologías de contactores. En la figura 2.5 se muestra una imagen en tres dimensiones de un contactor de corriente continua con los principales componentes que se han descrito anteriormente. Mientras que el contactor mostrado en la figura 2.6 representa en mayor detalle la construcción típica de un contactor de corriente alterna, de similares características al que se ensayará en la presente Tesis Doctoral.

En base al listado anterior de componentes y a la información mostrada 
en las figuras 2.5 y 2.6, para el funcionamiento del contactor los contactos principales se conectan a la carga eléctrica que se desee alimentar, ya sea una máquina eléctrica rotativa, una carga de iluminación, etc. Según el número de fases de alimentación que requiera dicho equipo, el contactor podrá ser bipolar, tripolar, tetrapolar, etc., realizándose las operaciones de apertura/cierre simultáneamente en todas las fases. Cuando la bobina del contactor se excite debido a la circulación de corriente eléctrica, la armadura comienza a desplazarse y arrastrar los contactos, hasta que se produce la unión entre la armadura y el núcleo. Cuando se suprime la tensión de alimentación en la bobina, los contactos se abren por efecto del resorte de los contactos y del resorte principal.

Continuando en la misma línea, en funcionamiento normal, debido al carácter inductivo de la bobina del contactor, la intensidad que la atraviesa se encuentra $90^{\circ}$ retrasada con respecto a la tensión en sus bornes. De esta manera, surge uno de los aspectos más importantes relacionados con el comportamiento del contactor ante las perturbaciones en las redes eléctricas. El diseño mecánico del contactor implica que, cuando se procede a la conexión del mismo, los contactos eléctricos se cierran antes de que la armadura haya finalizado su movimiento, tal y como indican Turner y Collins (1996); Collins y Bridgwood (1997) y muestra mediante simulaciones Riba et al. (2011). De igual manera, este diseño permite que el circuito magnético se desconecte parcialmente antes de que lo haga el eléctrico cuando se proceda a la desconexión del contactor. Por lo tanto, este comportamiento permite que el circuito magnético se pueda desconectar y volver a conectar sin llegar a la desconexión del circuito eléctrico, es decir, sin causar la desconexión de la carga eléctrica alimentada o la paralización del proceso industrial. En Tarczynski et al. (2003) se presenta un extenso estudio sobre el comportamiento interno de un contactor variando la tensión de alimentación así como el inicio del ángulo de la onda de dicha tensión. Con el mismo interés, en dos Santos Dias de Moraes y Perin (2008); Espinosa et al. (2008); Chieh-Tsung (2008); Chih-Yu y Chieh-Tsung (2010) se detalla el principio de funcionamiento teórico del contactor junto con valores típicos de parámetros.

De esta manera, durante un hueco de tensión, se procederá a la desconexión no deseada del contactor si la fuerza electromagnética ejercida por la bobina, $f_{\text {min }}$, es menor o igual que la fuerza ejercida por los resortes, $f_{\text {spring: }}$ :

$$
f_{\text {min }}=\frac{1}{2 \mu_{0} A} \phi_{m}^{2} \leq f_{\text {spring }}
$$

Donde $\mu_{0}$ es la permeabilidad del vacío, $A$ es la sección transversal del núcleo electromagnético, y $\phi_{m}$ es el flujo mínimo necesario para evitar la desconexión. El valor de $\phi_{m}$ es directamente proporcional a la intensidad que atraviese la bobina del contactor.

Aunque los elementos electrónicos están sustituyendo cada vez más a los 
electromecánicos, sobre todo en bajas potencias, Costello (2007), todavía se pueden encontrar en una gran cantidad de instalaciones ya que hoy en día son los más rentables económicamente para la mayoría de las aplicaciones. Por ejemplo, en aplicaciones industriales donde se utilicen elementos como cintas transportadoras, resulta muy habitual la conexión y desconexión de motores trifásicos una vez por segundo; por lo que el contactor asociado a dicha maniobra sufrirá el mismo ciclo de trabajo. El control electrónico en contactores, dejando de lado la adicional inversión económica así como la complejidad que necesita este tipo de contactor frente al electromagnético convencional, aporta una ventaja fundamental: para su operación soporta un amplio rango de valores de tensión, ya sea alterna o continua; mientras que el contactor convencional requeriría de varias bobinas para poder cumplir dicha función. Además, existen algunas tipologías que permiten solventar uno de los problemas más comunes a que se encuentran sometidos estos equipos, que es el desgaste que se produce por el choque de los contactos en cada operación del contactor, ya sea en el cierre de los contactos Espinosa et al. (2008), o en el momento de apertura de los mismos debido principalmente al arco eléctrico que se produce al proceder a su apertura, dos Santos Dias de Moraes y Perin (2008).

Igual que se hizo con los elementos del contactor, es importante citar los parámetros que definen a un contactor, dado que representan características técnicas que se emplearán más adelante. Por ello, en base a las definiciones dadas por UNE-EN 60947-4-1, se tienen en cuenta las siguientes características:

- Tensión nominal $V_{n}$ : representa la tensión que, combinada con una intensidad nominal, $I_{n}$, determina el uso del contactor en función de su categoría de empleo o utilización. En circuitos trifásicos se expresa generalmente por la tensión entre fases.

- Tensión nominal del circuito de control $V_{n c}$ : tensión aplicada en los terminales de la bobina.

- Tensión nominal de aislamiento $V_{i}$ : máximo valor de tensión que se puede aplicar al contactor en los contactos. En ningún caso $V_{n}$ puede ser superior a $V_{i}$.

- Intensidad nominal $I_{n}$ : se define en función de la tensión nominal $V_{n}$, la categoría de utilización y la temperatura ambiente del contactor. Es importante no confundir la intensidad nominal o de servicio, con el valor de la intensidad térmica $I_{t h}$. Generalmente $I_{n}$ es inferior a $I_{t h}$, y se define como la intensidad máxima que el contactor puede establecer, soportar e interrumpir, para las condiciones de uso establecidas, sin producir un calentamiento excesivo, ni un deterioro exagerado de los contactos. 
- Intensidad nominal térmica $I_{t h}$ : es la intensidad que puede soportar un contactor durante 8 horas sin que el aumento de temperatura producida por la misma supere los rangos de temperatura admisibles.

- Intensidad de cierre $I_{c}$ : valor máximo de intensidad que el contactor soporta en el instante inicial de conexión de forma satisfactoria, es decir sin riesgo de soldadura de sus contactos. Este valor resulta crítico en la elección de contactores para operación de cargas con fuertes intensidades de arranque. Está condicionado por el valor de la tensión de alimentación de la bobina del contactor, que es la que realiza la fuerza para mantener cerrado el contactor.

- Intensidad de corte: es el máximo valor de intensidad que el contactor puede interrumpir de forma satisfactoria para una tensión de empleo dada. Al producirse el corte de la corriente se genera un arco eléctrico; lo que constituye una de las principales causas de deterioro de los contactos. Este arco eléctrico genera altas temperaturas que pueden originar la fusión y/o volatilización del material de los contactos. Si la intensidad de corte es elevada, la extinción del arco se produce en condiciones difíciles y hasta imposible (duración elevada, expulsión de llamas, mantenimiento del arco, etc.).

Tal y como se ha visto en el listado anterior, uno de los parámetros importantes de los contactores es su categoría de empleo, la cual define la aplicación a la cual está destinado el contactor. Cada categoría de empleo se caracteriza básicamente por la intensidad que el contactor debe establecer o cortar. Por este motivo, un mismo contactor posee diferentes categorías de utilización. Por ejemplo, si se elige un contactor para maniobrar un motor equivaldría a una categoría AC-3-, el mismo contactor podría ser empleado para maniobrar una carga resistiva de mayor potencia - en este caso sería categoría AC-1- La Norma UNE-EN 60947-4-1 establece 12 categorías para corriente alterna y 4 categorías para corriente continua, representadas en la tabla 2.2 .

En relación con una de las aplicaciones donde se emplean contactores, como es en la maniobra de máquinas eléctricas rotativas, se puede afirmar que normalmente suele existir un relé térmico instalado aguas abajo del contactor, con el objeto de proteger el circuito de potencia contra sobrecargas; ya que el contactor por sí solo no es un elemento de protección, sino de maniobra.

Una vez que se conocen las características generales de los contactores se está en condiciones de comenzar a analizar el trabajo desarrollado por diferentes autores lo largo de los años dentro de la temática de la presente Tesis Doctoral. Aunque en Honrubia-Escribano et al. (2012a) se presenta un breve resumen, a continuación se desarrolla en mayor profundidad el 


\begin{tabular}{c|l}
\hline Categoría & Aplicación representativa \\
\hline AC-1 & Cargas no inductivas o ligeramente inductivas. \\
AC-2 & Motores de anillos rozantes. \\
AC-3 & Motores de jaula de ardilla. \\
AC-4 & Motores de jaula de ardilla (marcha intermitente). \\
AC-5a & Conmutación lámparas eléctricas de descarga. \\
AC-5b & Conmutación lámparas incandescentes. \\
AC-6a & Conmutación de transformadores. \\
AC-6b & Conmutación de bancos de condensadores. \\
AC-7a & Cargas ligeramente inductivas domésticas. \\
AC-7b & Cargas de motor domésticas. \\
AC-8a & Control motor compresor, rearme manual. \\
AC-8b & Control motor compresor, rearme automático. \\
\hline DC-1 & Cargas no inductivas o ligeramente inductivas. \\
DC-3 & Motores en derivación. \\
DC-5 & Motores serie. \\
DC-6 & Conmutación lámparas incandescentes. \\
\hline
\end{tabular}

Tabla 2.2: Categorías de empleo de contactores, UNE-EN 60947-4-1

estado del arte del comportamiento de contactores ante huecos de tensión e interrupciones del suministro.

El primer trabajo relevante en esta temática se encuentra en Turner y Collins (1996); Collins y Bridgwood (1997). Ensayan sólo un modelo de contactor, de $120 V_{a c}$ de tensión nominal del circuito de control — parámetro designado previamente por $V_{n c}$ (de aquí en adelante, si no se indica lo contrario, se hará referencia a $V_{n c}$ ) - donde afirma que el contactor no se desconecta para una tensión residual mínima del $40 \%$. El mismo autor, en otro trabajo ensaya un contactor de $220 V_{a c}$, situándose la tensión residual mínima en el $58 \%$, Collins y Zapardiel (1997). Aún tratándose de los primeros estudios, señalan que sólo los parámetros magnitud y duración del hueco de tensión no sirven para caracterizar la respuesta de contactores; ya que el ángulo de inicio de la onda de tensión es un parámetro a tener en cuenta. Además, se afirma que existe una simetría de cuarto de ciclo, es decir, que los resultados que se pueden obtener a $0^{\circ}$ de inicio de la onda de tensión son los mismos que se consiguen a $180^{\circ}$, por ejemplo. Igualmente, señalan que los contactores exhiben un comportamiento relativamente aleatorio ante huecos de tensión. Un año más tarde, el "Electric Power Research Institute (EPRI)", EPRI (1998), publica un breve informe sobre el resultado de huecos de tensión sobre un relé y tres contactores de diferente tamaño.

Sin embargo, han de pasar algunos años hasta que se realice un estudio 
más exhaustivo, Pohjanheimo y Lehtonen (2002); Djokic et al. (2004). En Djokic et al. (2004) son ensayados en vacío seis contactores de diferentes fabricantes y tensiones nominales del circuito de control donde, por un lado se vuelve a poner de manifiesto la compleja respuesta del contactor frente a huecos de tensión y, de manera similar, se vuelve a señalar el fenómeno de la simetría de cuarto de ciclo. Además, en Djokic et al. (2004) se hace un análisis de la influencia del salto de fase durante el hueco de tensión sobre el contactor. Teóricamente, el salto de fase máximo durante un hueco de tensión podría ser $90^{\circ}$ aunque, en la práctica, lo usual es que no sea superior a $45^{\circ}-60^{\circ}$, Bollen (2000). No obstante, la influencia del salto de fase sobre la respuesta del contactor no se aprecia sobre los contactores ensayados en Djokic et al. (2004). De igual manera, en Pohjanheimo y Lehtonen (2002) se ensayan varios contactores variando el inicio de la onda de tensión pero, en este caso, el estudio no permite profundizar en los resultados debido a las limitadas prestaciones del generador de huecos de tensión empleado.

Gracias a estos primeros trabajos, se dedujo una de las características más importantes en relación con la respuesta de contactores de corriente alterna frente a huecos de tensión e interrupciones, que es la elevada influencia del ángulo en el que se inicia el hueco. Esta afirmación implica que una interrupción del suministro (o un hueco de tensión de elevada profundidad) que se produzca a $90^{\circ}$ de inicio de la onda de tensión ocasiona el disparo del contactor con menores duraciones que si dicha perturbación se iniciase en un ángulo de $0^{\circ}$. Dicho de otra manera, la respuesta del contactor es más crítica - o más sensible - cuando la interrupción se inicia a $90^{\circ}$. Este comportamiento será ampliamente analizado en el apartado 2.4.1 en base al extenso desarrollo experimental llevado a cabo en la Tesis.

Posteriormente al trabajo desarrollado por Djokic et al. (2004), en Kushare y Ghatol (2007) se obtiene la curva de tolerancia de tres contactores de diferentes características. Nuevamente se incide en la simetría de la respuesta del contactor aunque, adicionalmente se indica que la incorporación de un condensador junto a la bobina del contactor permite mejorar la respuesta del mismo ante huecos de tensión. Sin embargo, debido a la baja calidad de las figuras, no se puede extraer ningún valor numérico de su trabajo. Por otro lado, aunque en Iyoda et al. (2007) sólo se realicen un número reducido de ensayos sobre un tipo de contactor, de $100 V_{a c}$, se manifiesta la dependencia de la respuesta del mismo con respecto al ángulo de inicio del hueco de tensión. Concretamente, muestra que el contactor, ante un hueco del $100 \%$ de profundidad — técnicamente, este fenómeno se llamaría "interrupción"-, dispara en tan solo $10 \mathrm{~ms}$ si el inicio de la onda de tensión se produce en $90^{\circ}$ mientras que se amplía a $65 \mathrm{~ms}$ si la onda de tensión se inicia en $0^{\circ}$.

Un aspecto que ninguno de los trabajos anteriores había tenido en cuenta era el efecto de huecos de tensión consecutivos debidos, por ejemplo, al efecto del "reclosing" automático que se comentó en el apartado 2.1.1. Por ello, en 
Moon et al. (2007) se ensayan varios tipos de cargas ante diversos huecos de tensión, tanto individuales como consecutivos, concluyendo que el efecto de los huecos de tensión consecutivos sobre la variada aparamenta eléctrica ensayada resulta ser muy similar al de los huecos que ocurren de manera aislada.

Las publicaciones más recientes apuntan hacia las mismas conclusiones. En Shareef et al. (2010a); Hardi y Daut (2010); Akolkar y Kushare (2010) se obtienen las curvas de tolerancia de varios contactores con igual tensión nominal de bobina y diferentes intensidades nominales; aunque la curva ensayada en Shareef et al. (2010a) posee intervalos de tiempo demasiado grandes (1 ciclo). También, en Akolkar y Kushare (2010) se indican las posibles mejoras para soportar huecos de tensión, como serían las fuentes de alimentación adicionales o los condensadores. En todas ellas se vuelve a señalar la influencia del inicio de la onda de tensión en la curva característica. Aún tratándose de referencias recientes, Akolkar y Kushare (2010) señala textualmente que "la respuesta de contactores ante huecos de tensión es muy compleja y difícil de predecir".

Además de los trabajos experimentales citados anteriormente, también se han realizado estudios de la respuesta de contactores en base a modelos de simulación. En Mohamad y Nor (2004) se muestra la respuesta de un contactor ante huecos de tensión mediante un modelo desarrollado bajo la herramienta PSCAD/EMTDC. Aún tratándose de un análisis posterior a los primeros trabajos experimentales realizados por otros autores, en su modelo no incluye el parámetro de inicio de la onda de tensión. Por este motivo, en la misma Universidad, Ismail et al. (2010) desarrolla un nuevo modelo de simulación que permite la variación del ángulo de inicio de la tensión. En cualquier caso, los resultados de ambas simulaciones, Mohamad y Nor (2004); Ismail et al. (2010), difieren de los resultados experimentales que han aportado otros autores. Sin embargo, en Pedra et al. (2002); Jeong et al. (2009); Hannan et al. (2012), se presentan modelos de simulación del contactor con elevada precisión. En estos últimos trabajos mencionados sobre modelado se demuestra también la importancia del inicio de la onda de tensión, y los resultados obtenidos son muy similares a los conseguidos por otros autores en experimentos reales.

Para sintetizar la información ofrecida en los párrafos anteriores, la tabla 2.3 recopila las tensiones mínimas de control, $V_{n c_{m i n}}$, que se han obtenido en base a la extensa revisión bibliográfica realizada. Esta tensión mínima de control implica que cualquier hueco de tensión que posea una tensión residual por encima de dicho valor no causará la desconexión del contactor. Con el mismo interés, se indica el tiempo máximo, $t_{\max }$, que soporta el contactor ante una interrupción del suministro. Dado que ha sido revisada la influencia del inicio de la onda del hueco de tensión en el comportamiento del contactor, la tabla 2.3 muestra $V_{n c_{\min }}$ y $t_{\max }$ en los casos extremos, es decir, para 


\begin{tabular}{|c|c|c|c|c|c|}
\hline Referencia & $V_{n c}$ & $V_{n c_{m i n}{ }_{90} 0}$ & $V_{n c_{m i n}{ }_{0} 0}$ & $t_{\max _{90} 0}$ & $t_{\max _{0^{0}}}$ \\
\hline Collins y Bridgwood (1997) & $120 V_{a c}$ & $43 \%$ & $62 \%$ & $12 \mathrm{~ms}$ & $30 \mathrm{~ms}$ \\
\hline Collins y Zapardiel (1997) & $220 V_{a c}$ & $54 \%$ & $59 \%$ & $10 \mathrm{~ms}$ & $30 \mathrm{~ms}$ \\
\hline Pohjanheimo y Lehtonen (2002) & - & $45 \%$ & $50 \%$ & $40 \mathrm{~ms}$ & $130 \mathrm{~ms}$ \\
\hline Djokic et al. 2004) & $24 V_{a c}$ & $65 \%$ & $72 \%$ & $8 \mathrm{~ms}$ & $80 \mathrm{~ms}$ \\
\hline 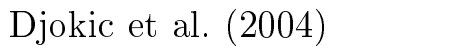 & $110 V_{a c}$ & $39 \%$ & $43 \%$ & $6 \mathrm{~ms}$ & $81 \mathrm{~ms}$ \\
\hline 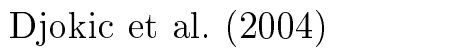 & $110 V_{a c}$ & $33 \%$ & $37 \%$ & $8 \mathrm{~ms}$ & $98 \mathrm{~ms}$ \\
\hline Djokic et al. & $110 V_{a c}$ & $40 \%$ & $46 \%$ & $7 \mathrm{~ms}$ & $110 \mathrm{~ms}$ \\
\hline$\overline{\overline{D j o k i c} \text { et al. }}(\overline{\overline{2004}})$ & $240 V_{a c}$ & $40 \%$ & $49 \%$ & $6 \mathrm{~ms}$ & $115 \mathrm{~ms}$ \\
\hline Djokic et al. $(\overline{2004})$ & $240 V_{a c}$ & $40 \%$ & $45 \%$ & $9 \mathrm{~ms}$ & $82 \mathrm{~ms}$ \\
\hline Mohamad y Nor (2004) & $240 V_{a c}$ & \multicolumn{2}{|c|}{$54 \%$} & \multicolumn{2}{|c|}{$50 \mathrm{~ms}$} \\
\hline Moon et al. (2007) & $220 V_{a c}$ & \multicolumn{2}{|c|}{$60 \%$} & \multicolumn{2}{|c|}{$10 \mathrm{~ms}$} \\
\hline Jeong et al. (2009) & $220 V_{a c}$ & $55 \%$ & $60 \%$ & is & 50 \\
\hline Shareef et al. 2010a & $240 V_{a c}$ & $55 \%$ & $65 \%$ & $10 \mathrm{~ms}$ & $80 \mathrm{~ms}$ \\
\hline Shareef et al. $(\overline{2010 a})$ & $240 V_{a c}$ & $47 \%$ & $63 \%$ & $10 \mathrm{~ms}$ & $80 \mathrm{~ms}$ \\
\hline Shareef et al. (2010a) & $240 V_{a c}$ & $47 \%$ & - & $10 \mathrm{~ms}$ & $160 \mathrm{~ms}$ \\
\hline Shareef et al. (2010a) & $240 V_{a c}$ & $55 \%$ & $57 \%$ & $10 \mathrm{~ms}$ & $80 \mathrm{~ms}$ \\
\hline Hardi y Daut 2010 & $240 V_{a c}$ & $50 \%$ & $50 \%$ & $10 \mathrm{~ms}$ & $70 \mathrm{~ms}$ \\
\hline Hardi y Daut $2 \overline{2010}$ & $240 V_{a c}$ & $40 \%$ & $30 \%$ & $40 \mathrm{~ms}$ & $90 \mathrm{~ms}$ \\
\hline Hardi y Daut $(\overline{2010})$ & $240 V_{a c}$ & $20 \%$ & - & $20 \mathrm{~ms}$ & $200 \mathrm{~ms}$ \\
\hline
\end{tabular}

Tabla 2.3: Resumen de los principales estudios realizados sobre huecos de tensión en contactores con diferentes ángulos de inicio de la onda de tensión.

$90^{\circ}$ y para $0^{\circ}$.

Observando la tabla 2.3 se pueden obtener relevantes conclusiones de los trabajos desarrollados por diferentes autores hasta la fecha. Cuando existan huecos de tensión de larga duración, lo más perjudicial para el contactor es que la onda de tensión se inicie en $0^{\circ}$, ya que el parámetro $V_{n c_{\min 0_{0}}}$ es superior al parámetro $V_{n c_{m i n} 0_{00}}$ en todas las referencias, excepto en Hardi y Daut (2010). Por el contrario, de manera general, huecos de tensión profundos e interrupciones ocasionan el disparo del contactor a duraciones menores cuando el inicio de la onda de tensión se produzca a $90^{\circ}$ que cuando se genere a $0^{\circ}$, dado que el parámetro $t_{\max _{90^{0}}}$ es inferior al $t_{\max _{0^{0}}}$ en todas las referencias consultadas. Igualmente, es necesario tener en cuenta que algunas publicaciones muestran resultados de una manera demasiado sencilla, concretamente en Pohjanheimo y Lehtonen (2002) no se especifica el tipo de contactor ensayado, y Mohamad y Nor (2004); Moon et al. (2007) no 
especifican el inicio de la onda de tensión en los experimentos. Además, en Hardi y Daut (2010) se obtienen unos resultados demasiado diferentes a los que obtuvieron el resto de autores.

Por el motivo anterior, junto a la complejidad de la respuesta de este tipo de aparamenta puesta de manifiesto explícitamente por Turner y Collins (1996); Collins y Bridgwood (1997); Collins y Zapardiel (1997); Djokic et al. (2004); Akolkar y Kushare (2010), en la presente Tesis Doctoral se ha decidido profundizar y realizar una amplia variedad de ensayos. Concretamente, más de 40.000 ensayos practicados sobre contactores de corriente alterna son resumidos en el apartado 2.4.1.

\subsubsection{Análisis del relé de falta a tierra}

Los equipos diseñados para la detección e interrupción de faltas eléctricas a tierra han sido comúnmente empleados en sistemas de distribución en baja tensión desde los años 70, Coyle (2002). Este tipo de aparamenta eléctrica apareció debido a que estas faltas no eran fácilmente detectadas por los fusibles ni por los interruptores automáticos y podían causar la destrucción del equipamiento eléctrico. El funcionamiento de la mayoría de los relés de falta a tierra es el mismo. Se basan en la medida del vector suma de las intensidades que atraviesan el Transformador de Intensidad ("Current Transformer (CT)" que, en condiciones normales, debe ser igual a cero. Si el vector suma es diferente de cero, se compara con un valor límite - conocido normalmente como "pickup setting"- $\mathrm{y}$, si lo sobrepasa, el relé causa el disparo del interruptor automático asociado. El tiempo de actuación suele estar situado entre $100 \mathrm{~ms}$ y $500 \mathrm{~ms}$.

Por lo tanto, la función principal de los relés de falta a tierra es la medida de la corriente de fuga a tierra de una instalación eléctrica e interrumpir el suministro si la corriente de falta a tierra supera el "pickup setting". La figura 2.7 muestra un diagrama de conexión típico de este tipo de aparamenta donde se observan cada uno de los elementos nombrados en las líneas anteriores.

Teniendo en cuenta que alrededor del $85 \%$ de los cortocircuitos que se producen, se manifiestan inicialmente como faltas a tierra, David et al. (1993)

-incluso en la industria petroquímica este valor puede ascender al $90 \%$, NelSon (2002) —, la detección de este tipo de faltas en el menor tiempo posible puede evitar dañar otros equipos industriales y aumentar la seguridad de las personas. En este caso, los equipos de detección de faltas a tierra son capaces de localizar una falta a tierra antes de que cause fibrilación ventricular en seres humanos. De hecho, con el objeto de aumentar la seguridad de las personas, se vienen empleado diferentes métodos como la instalación de resistencias de tierra, incorporación detectores de falta a tierra, así como la monitorización de tierra, Novak et al. (1988). Las redes eléctricas con sistemas de resistencia a tierra de altos valores limitan la máxima corriente 


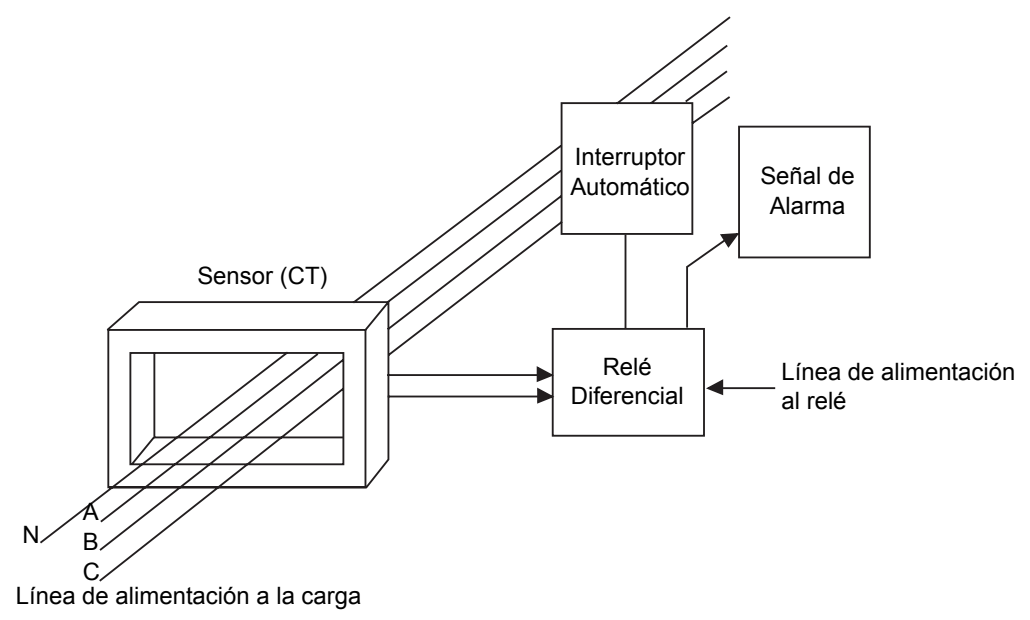

Figura 2.7: Sistema de protección por relé de falta a tierra.

de falta a tierra de tal manera que no se produzca la fibrilación ventricular. Además, en redes de media tensión, los sistemas de resistencia de tierra de elevados valores permiten la operación normal sin disparo del proceso industrial durante un tiempo indefinido; con lo que es posible planear la interrupción programada del mismo para proceder a su reparación, Kingrey et al. (2011). Por el contrario, valores altos en la resistencia de tierra ocasionan que la corriente de falta sea reducida y, por tanto, el relé de falta a tierra no actúe y sea directamente el interruptor automático el que dispare la línea después de un largo periodo de tiempo.

Teniendo en cuenta las anteriores premisas, es importante tener en cuenta que la presencia de huecos de tensión causan un funcionamiento incorrecto del equipo de protección poniendo en peligro tanto a equipos eléctricos como a personas; dado que leves faltas a tierra pueden acabar transformándose en elevadas corrientes de falta, Zielichowski y Szlezak (2007). Además, dado que no se han encontrado en la bibliografía científica trabajos que evalúen el comportamiento de este tipo de aparamenta eléctrica ante huecos de tensión, se ha motivado su ensayo en la presente Tesis Doctoral. Concretamente, se ha ensayado un tipo de relé de falta a tierra comúnmente empleado en el sector industrial.

\subsubsection{Análisis del variador de frecuencia}

Los variadores de frecuencia - conocidos como "Adjustable Speed Drive (ASD)" o "Variable Frequency Drive (VFD)"- se emplean para controlar casi cualquier tipo de carga que esté compuesta por un motor, tanto de corriente alterna como continua, ya sea un pequeño ventilador o un gran motor industrial. De hecho, su rango de empleo varía desde potencias inferiores a 


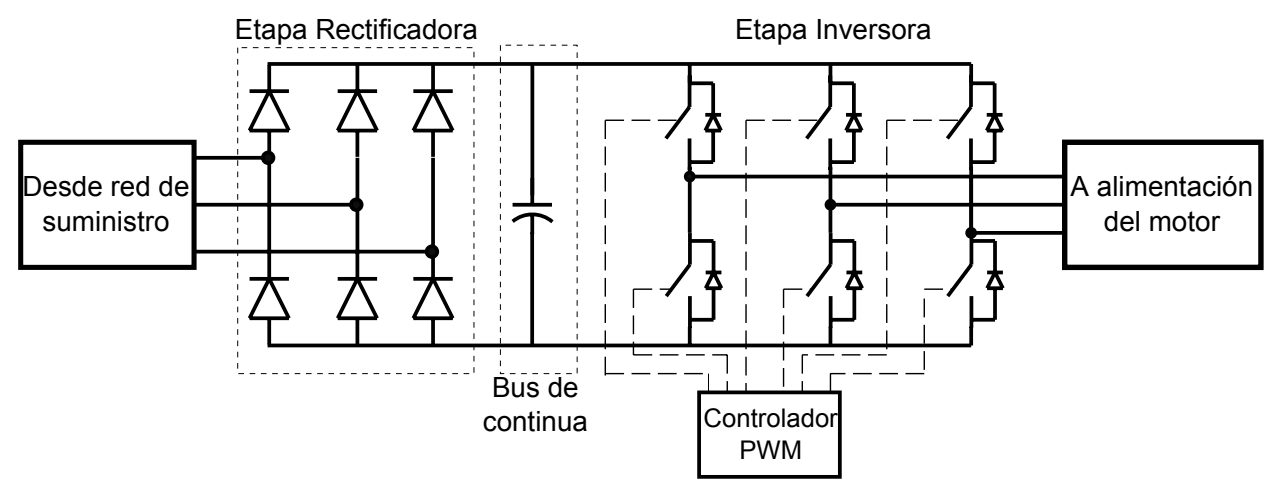

Figura 2.8: Esquema constructivo de un variador de frecuencia.

un vatio hasta cientos de megavatios, Boldea (2008); Abu-Rub et al. (2010).

En esencia, los variadores de frecuencia están compuestos por una etapa rectificadora que se conecta a directamente a la red eléctrica, un bus de continua que suaviza la salida de la parte rectificadora, y una etapa inversora de frecuencia variable que se conecta a la carga a controlar. La figura 2.8 muestra el diagrama de construcción típico de un variador de frecuencia. El bus de continua puede estar constituido sólo por condensadores aunque, en ocasiones, también se añaden reactancias para suavizar la intensidad del rectificador así como reducir la distorsión armónica. Según la bibliografía consultada, el valor de la capacidad del bus de continua se sitúa entre 75 $360 \mu F / k W$, Bollen y Zhang (2000), o entre $59-180 \mu F / k W$, Stockman et al. (2003).

Este tipo de aparamenta eléctrica genera uno de los problemas más importantes asociados con los huecos de tensión. Además de ser particularmente sensibles a este tipo de perturbaciones electromagnéticas, su elevada potencia nominal causa que los métodos de mitigación de los efectos de las perturbaciones resultan ser demasiado complejos técnicamente y costosos económicamente, Baggini (2008). En muchos procesos industriales, una variación mínima de la velocidad o del par de un motor no se puede tolerar, incluso cuando el tiempo de actuación sea muy reducido. Por ejemplo, en el caso de fábricas de papel y textiles, un hueco de tensión de poca profundidad puede causar que el variador de frecuencia introduzca variaciones de velocidad que dañen el producto final, Duran-Gomez et al. (1999). En otros tipos de cargas, la precisión de la velocidad y del par puede no resultar un parámetro determinante, como sería el caso de aspiradores, ventiladores, etc. Otro factor a tener en cuenta es que su sensibilidad frente a huecos de tensión se encuentra íntimamente relacionada con la tipología de construcción empleada por cada fabricante, Sarmiento y Estrada (1996); Duran-Gomez et al. (1999); Djokic et al. (2005b). 
Cuando un variador de frecuencia es perturbado por un hueco de tensión se pueden generar, tanto sobreintensidades en la etapa rectificadora que posiblemente ocasionen el disparo del equipo, así como una disminución de la tensión de la etapa de continua causando variaciones sobre el par de la máquina que se está controlando. Concretamente, cuando el hueco incide en una fase, el par de diodos asociados a la misma se polariza de manera inversa, por lo que éstos dejan de permitir el paso de corriente y, por lo tanto, de esa fase deja de extraerse corriente eléctrica. Bajo este supuesto, la respuesta del variador de frecuencia no va a depender de la magnitud del hueco de tensión, Duran-Gomez et al. (1999); Bollen y Zhang (2000). De esta manera, se produce tanto la disminución de la tensión en el bus de continua - según Bollen y Zhang (2000), esta circunstancia es la principal causa de disparo en estos equipos-, como un aumento de la intensidad consumida por las otras dos fases que no se encuentran en falta — según Rocha y Madrigal (2005) los variadores de frecuencia soportan intensidades máximas entre $1.2 \mathrm{pu} \mathrm{y}$ $1.5 \mathrm{pu}$, aunque también pueden llegar a $1.7 \mathrm{pu}$, Djokic et al. (2005b), o incluso $2.4 \mathrm{pu}$, Djokic et al. (2008) - . Si el hueco de tensión es demasiado profundo, el par electromagnético de la carga asociada al variador de frecuencia puede resultar inferior al par mecánico del proceso industrial; lo que conlleva una reducción de la velocidad del motor. Por lo tanto, aunque la parte eléctrica del variador de frecuencia pueda soportar el hueco de tensión sin producir un disparo, existen otros factores externos al equipo, como la disminución de la velocidad de la carga. Cuando el hueco de tensión desaparece y la tensión recobra su estado nominal, un reinicio automático del variador de frecuencia no suele ser posible debido a que la etapa inversora podría no estar en sincronismo con las tensiones residuales en el estator; por lo que podría resultar necesario un rearme manual del proceso, Djokic et al. (2005b); Carrillo et al. (2010).

Con resultados similares, si el hueco de tensión que perturba al variador de frecuencia es trifásico equilibrado, en el momento en que la tensión de alimentación del variador es inferior a la rectificada en el bus de continua, se producirá la descarga progresiva del condensador dado que no se produce absorción de corriente desde la red, Belchior et al. (2003). La tensión en el condensador continuará disminuyendo con la consiguiente disminución de la velocidad de giro del motor controlado hasta que se llegue al valor de tensión mínima de funcionamiento del variador, $V_{d c_{m i n}}$, momento en que se producirá el disparo del mismo. $V_{d c_{\text {min }}}$ suele ser un parámetro definido por el usuario, aunque los valores de fábrica se sitúan entre el 60 - $85 \%$ de la tensión nominal del bus de continua, $V_{d c_{n o m}}$, Stockman et al. (2003); Deswal et al. (2010); Bollen et al. (2010), llegando en algunos casos hasta el 50\%, Djokic et al. (2005b, 2008). Es importante tener en cuenta que, en estas condiciones, la descarga del condensador no depende de la magnitud del hueco de tensión, sino de la carga que está alimentando, Bollen y Zhang (2000). 


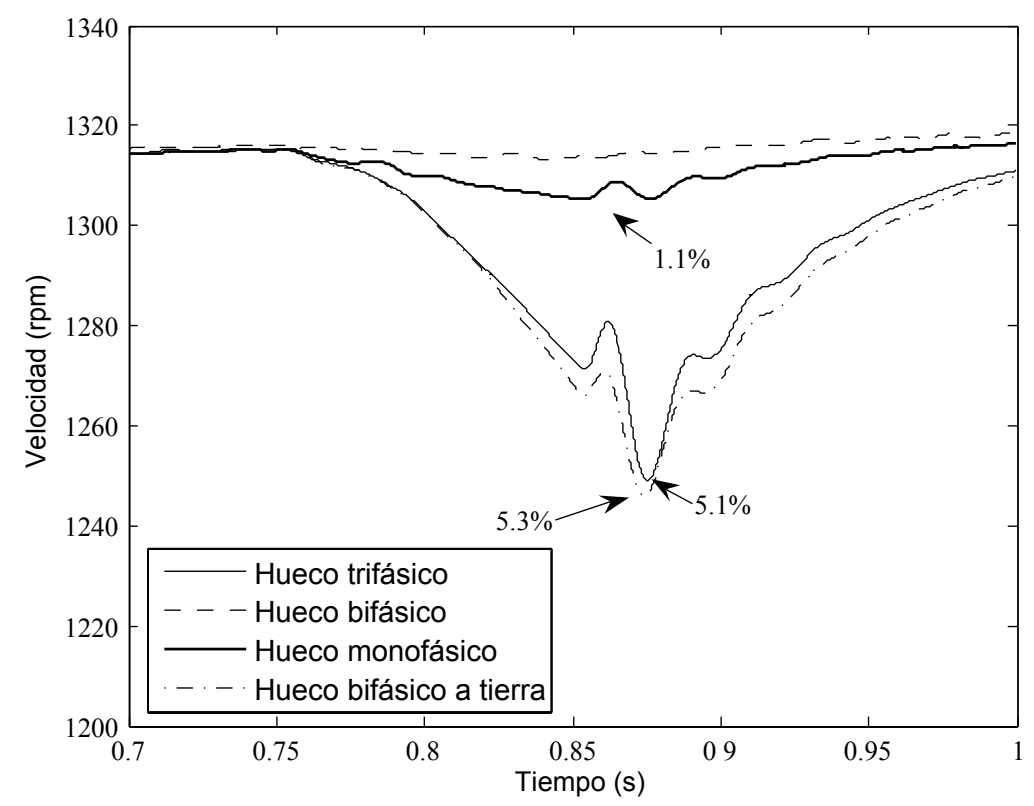

Figura 2.9: Variación de la velocidad de un motor en función del hueco de tensión aplicado sobre el variador de frecuencia, Rocha y Madrigal (2005).

Para comprender con mayor claridad el comportamiento del variador de frecuencia ante perturbaciones en la red eléctrica tal y como se ha resumido en los dos párrafos anteriores, se presentan las figuras 2.9 y 2.10 . La figura 2.9 resume el efecto de los huecos de tensión de diversa tipología sobre la velocidad del motor controlado por un variador de frecuencia trifásico, en base a los resultados simulados en Rocha y Madrigal (2005), para un hueco de tensión de $100 \mathrm{~ms}$ de duración comenzando a los $0.75 \mathrm{~s}$ de simulación. Como se ha señalado, la disminución progresiva de la velocidad de giro del motor es el resultado de la descarga del condensador del bus de continua hasta el momento en que la tensión de alimentación de la red eléctrica carga nuevamente al condensador. La reducción en la velocidad producida por los huecos monofásico y bifásico es menor del $1.5 \%$; mientras que en los huecos trifásico y bifásico a tierra se supera el $5 \%$ de variación de la velocidad. Las simulaciones llevadas a cabo en Pedra et al. (2005); Vegunta et al. (2006); Djokic et al. (2008); Ramela y Kumar (2011) indican idénticos resultados, afirmando que los huecos trifásicos producen la peor respuesta del variador de frecuencia; mientras que los huecos bifásicos y monofásicos son los mejor soportados por el equipo. Igualmente, los resultados experimentales de Djokic et al. (2005b) apuntan en la misma dirección.

Por otro lado, la figura 2.10 muestra el comportamiento del variador de frecuencia ante huecos de tensión trifásicos equilibrados de $200 \mathrm{~ms}$ de duración, en base a la simulación realizada en O'Connell y Kirawanich 2005). 


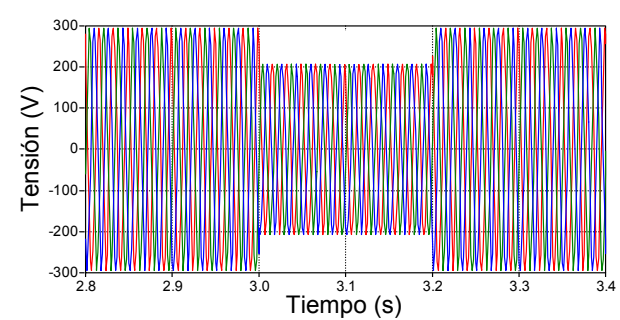

(a) Tensión de alimentación del variador.

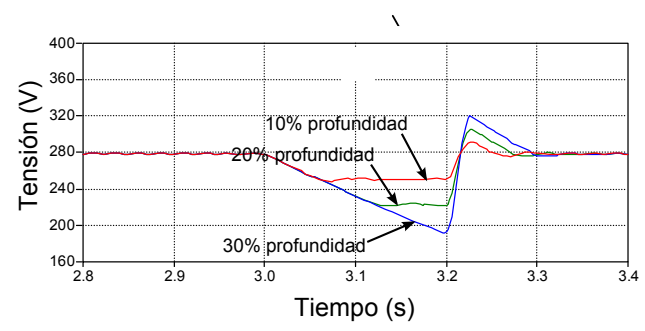

(c) Tensión en el bus de continua.

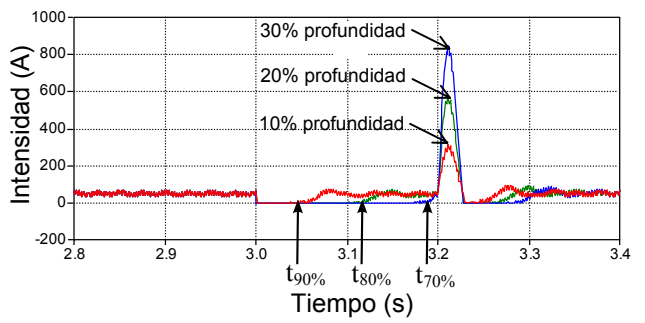

(b) Intensidad absorbida por el variador.

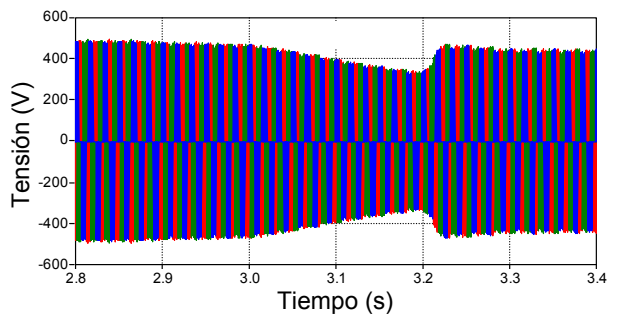

(d) Tensión de salida del variador.

Figura 2.10: Comportamiento del variador de frecuencia ante un hueco de tensión trifásico equilibrado, O'Connell y Kirawanich (2005).

En la figura 2.10a se muestra la tensión de alimentación del variador. Debajo de ésta se encuentra la variación de la tensión en el bus de continua en función de la tensión residual (se contempla la simulación de tres huecos de tensión de diferente profundidad), subfigura (c). Se observa que la tensión en el bus de continua disminuye progresivamente hasta que su tensión se iguala con la de la red de alimentación (caso de los dos huecos menos severos) o hasta que se recupera el hueco (caso del hueco de mayor profundidad). En la figura $2.10 \mathrm{~b}$ se muestran las intensidades de alimentación al variador en función de la profundidad del hueco. Se observa claramente como, cuando la tensión en la etapa de continua es superior a la de alimentación, el variador deja de absorber intensidad de la red dado que los diodos se encuentran polarizados inversamente y, lo que aún resulta más importante es observar la elevada intensidad absorbida en el momento en que se restaura la tensión de alimentación. Por último, el gráfico (d) de la figura 2.10 muestra el valor de la tensión trifásica aplicada sobre el motor controlado por el variador. Como se ha comentado anteriormente, esta disminución de la tensión de alimentación del motor ocasiona que se reduzca el par electromagnético del mismo y, por lo tanto, la velocidad del proceso industrial asociado resulte modificada.

La limitación de las sobreintensidades así como el mantenimiento de la tensión del bus de continua, es decir, la mejora de la respuesta de este equipo ante huecos de tensión se puede efectuar de diferentes maneras:

- Para variadores de frecuencia cuyo bus de continua está constituido por 
un condensador: un descenso de la capacidad del condensador origina que los picos de intensidad se reduzcan, aunque de manera muy limitada, Rocha y Madrigal (2005). Sin embargo, esta solución causa mayores fluctuaciones de tensión en la etapa de continua cuando se perciba un hueco de tensión. Concretamente, en Bollen y Zhang (2000) se detalla cómo el aumento de la capacidad del condensador disminuye la variación de la tensión en el bus de continua. Igualmente, en Belchior et al. (2003) se analiza el elevado pico de intensidad que aparece justo cuando se recupera la tensión nominal después de un hueco de tensión que no consigue el disparo del variador cuando la capacidad del condensador es elevada, fenómeno mostrado también en la figura 2.10b. De hecho, esta elevada intensidad de recuperación se encuentra condicionada por el parámetro $V_{d c_{\text {min }}}$.

- Para variadores de frecuencia cuyo bus de continua está constituido por filtro LC: un incremento del producto $L C$ consigue suavizar el rizado de la corriente en el rectificador, Vegunta et al. (2006). La principal desventaja de esta solución es su elevado coste, Deswal et al. (2009, 2010). La mayoría de los fabricantes de variadores de frecuencia con una potencia nominal superior a $100 \mathrm{~kW}$ emplean este método; al igual que muchos con potencia nominal a $10 \mathrm{~kW}$, David et al. (1993).

- Incorporación de un volante de inercia: esta opción de mejora de la respuesta de variadores de frecuencia frente a huecos de tensión implica la adición de una inercia adicional a la carga que controle el variador. Se persigue minimizar la reducción de la velocidad de giro del sistema, aunque la complejidad constructiva y económica de la instalación aumenta considerablemente, Belchior et al. (2003). Sin embargo, esta solución es viable para aplicaciones que empleen un rango de potencia situado entre 1 y $10 \mathrm{~kW}$, Deswal et al. (2009).

- Incorporación de un sistema regenerativo - conocido como "active front end"-: consiste en sustituir el puente de diodos — conocido como "passive front end"- por un puente rectificador activo (IGBTs), Vegunta et al. (2006), con el objeto de que la carga del bus de continua se pueda realizar en un mayor margen de tensiones de alimentación. De esta manera, el parámetro determinante de la sensibilidad del variador de frecuencia ante huecos de tensión es la intensidad que absorberá el rectificador activo. Cuanto más se quiera reducir $V_{d c_{m i n}}$, mayor intensidad deberá soportar el rectificador activo; al mismo tiempo que aumenta el coste económico, Stockman et al. (2003). De hecho, esta solución se emplea mayoritariamente en variadores de frecuencia de alta potencia nominal, empleando la opción con puente de diodos en bajas y medianas potencias, Djokic et al. (2008). 
- Incorporación de un convertidor elevador: esta solución conlleva la conexión de un convertidor elevador, que puede estar situado entre la etapa de continua y la etapa inversora del variador de frecuencia, Stockman et al. (2003), o entre la etapa rectificadora y el bus de continua, Deswal et al. (2011). De esta manera, en condiciones normales la tensión en el bus de continua equivale a $V_{d c_{n o m}}$ y el convertidor elevador no actúa. Al producirse un hueco de tensión, la tensión en el bus de continua comienza a disminuir hasta que llega un momento en que se activa el convertidor elevador y consigue mantener dicha tensión. De esta manera, el variador de frecuencia puede soportar huecos de tensión del $50 \%$ de profundidad y duración de 2 segundos, Stockman et al. (2003); Deswal et al. (2009). Existen variadores de frecuencia que incorporan de serie el convertidor elevador, aunque este elemento también puede ser acoplado sobre un variador que no lo posea previamente, Deswal et al. (2011). Continuando con esta línea, algunos trabajos han empleado un convertidor reductor-elevador - conocido como "buck-boost converter" - para, además de mejorar la respuesta del equipo ante huecos de tensión, protegerlo ante aumentos de la misma, Ramela y Kumar (2011).

Además del listado anterior de métodos de mejora de la capacidad de los variadores de frecuencia de soportar perturbaciones de las redes eléctricas, existen algunos fabricantes que incorporan diversas opciones configurables por el usuario que mejoran la respuesta ante huecos de tensión, Carrillo et al. (2010). Por ejemplo, para eliminar las elevadas intensidades que atraviesan el variador cuando se restaura la tensión después del hueco, existe una opción conocida como "arranque en vuelo" — denominado "flying start"consistente en que el variador de frecuencia desconecta la carga que alimenta mientras exista un hueco y, cuando éste desaparezca, el variador detecta cuál es la velocidad de giro del motor y comienza a controlarla hasta su valor nominal de manera gradual.

Además, recientemente han aparecido nuevas topologías de variadores de frecuencia. Por un lado existen los que se difieren de los convencionales básicamente en la disposición de elementos dentro del bus de continua y en la incorporación de condensadores en la etapa rectificadora, Bo et al. (2011), con el objetivo de mejorar la respuesta de esta aparamenta ante huecos de tensión. También existen otros autores que añaden nuevas etapas rectificadoras para que actúen manteniendo la tensión constante en el bus de continua en el momento en que se produce un hueco de tensión, Costa et al. (2011).

Una vez comprendidas las características y el comportamiento de los variadores de frecuencia ante diversas perturbaciones de las redes eléctricas, resulta de vital importancia conocer los resultados obtenidos por otros autores, ya sea de manera experimental o mediante simulaciones, sobre la 
tolerancia de esta aparamenta eléctrica ante las perturbaciones.

Uno de los primeros trabajos que evalúa el comportamiento de variadores de frecuencia ante huecos de tensión también presenta un breve estudio de la calidad de suministro, Sarmiento y Estrada (1996). Concretamente, después de monitorizar dos industrias durante un periodo de 17 meses, concluyeron que huecos de tensión con una duración mayor de 12 ciclos y una profundidad superior al $20 \%$ causaban el disparo de los variadores de frecuencia. Similares resultados obtuvo Keus et al. (1999), donde después de ensayar un variador de frecuencia bajo diferentes regímenes de carga se concluye que el efecto del régimen de carga es reducido y la desconexión del equipo se produce para profundidades superiores al $10 \%-20 \%$ y tiempos entre $20 \mathrm{~ms}$ y $50 \mathrm{~ms}$.

En Stockman et al. (2003) se ensayan varios tipos de variadores de frecuencia, con una potencia nominal comprendida entre $2.2 \mathrm{~kW}$ y $7.5 \mathrm{~kW}$, ante huecos de tensión. Primeramente, ensayando un variador de frecuencia con un valor reducido de la capacidad del bus de continua muestra que el variador dispara para profundidades de hueco superiores al $10 \%$; mientras que el tiempo que soporta una interrupción de la alimentación se sitúa entre $3 \mathrm{~ms}$ y $15 \mathrm{~ms}$ (el menor tiempo corresponde al mayor régimen de carga del equipo). Posteriormente, ensayando un variador de frecuencia de mayor capacidad del bus de continua se observa una notable mejora en el tiempo que puede soportar el equipo bajo una interrupción, ampliando el intervalo a 20 ms-50 ms. Además, muestra ensayos realizados con sistemas regenerativos y convertidores elevadores conectados a variadores de frecuencia comerciales.

En Belchior et al. (2003) se modela el variador de frecuencia para analizar la sensibilidad del variador ante huecos de tensión en función de varias soluciones, como el aumento de la capacidad del bus de continua y el acopio de una inercia adicional.

Sin embargo, hay que esperar hasta Djokic et al. (2005b) para encontrar un extenso trabajo experimental. De hecho, ensaya cinco tipos de variadores de frecuencia, de diferentes potencias nominales, capacidades de la etapa de continua, y $V_{d c_{n o m}}$, donde la primera afirmación que realiza es que este tipo de aparamenta muestra una respuesta demasiado compleja ante perturbaciones de red. Una de las conclusiones más importantes es que el punto de inicio de la onda de tensión no tiene influencia sobre la respuesta de variadores de frecuencia ante huecos de tensión (contrariamente al caso de los contactores mostrado en el apartado 2.2.1. También, en línea con lo mostrado en la figura 2.9, el ensayo frente a huecos de tensión monofásicos realizado en Djokic et al. (2005b) demuestra, de manera experimental, la mejor respuesta del equipo; llegando incluso a darse la situación en dos de los cinco variadores de frecuencia ensayados de que el hueco de tensión monofásico no causa el disparo del equipo. Además, presenta ensayos con variación de la velocidad del motor y, aunque los experimentos no muestran una clara tendencia, se observa una ligera disminución de la tensión residual soportada por el varia- 
dor de frecuencia cuando el motor gira a menor velocidad. En este sentido, Petronijevic et al. (2005) simula un variador de frecuencia en el que un hueco de tensión no afecta al comportamiento del mismo cuando el motor que alimenta está girando a baja velocidad.

Además de trabajos experimentales, Djokic et al. (2008) también realiza simulaciones en esta temática bajo la herramienta MatLab/SIMULINK, mostrando que incrementando el límite de intensidad máxima que puede atravesar el variador de frecuencia, el equipo mejora ligeramente su respuesta frente a huecos de tensión.

El trabajo desarrollado en Duran-Gomez et al. (1999), tanto analítico como experimental, aporta relevantes resultados. Ensaya dos tipos de variadores de frecuencia, uno con bus de continua constituido sólo por el condensador y otro que añade una inductancia. Demuestra que al añadir la bobina en el bus de continua aumenta la sensibilidad del variador ante huecos de tensión porque aumenta la variación de tensión en el bus de continua y, por tanto, se produce el disparo del equipo a menores profundidades de hueco que si no tuviese dicho elemento inductor. Además, en línea con Keus et al. (1999); Stockman et al. (2003); Pedra et al. (2005); Djokic et al. (2005b); Vegunta et al. (2006), se demuestra que el régimen de carga incluye sobre la respuesta del equipo, concluyendo que a mayores regímenes de carga se aumenta la sensibilidad del variador debido a una mayor reducción de la tensión del bus de continua y a mayores sobreintensidades.

En David et al. (1993) se presenta un control del variador de frecuencia basado en la variación del índice de modulación del variador de frecuencia a raíz de la medida de la tensión del bus de continua para soportar los huecos de tensión. Sin embargo, otros tipos de variadores de frecuencia utilizan la medida de la tensión entre dos de las fases de alimentación para disparar el equipo por baja tensión, Keus et al. (1999); Djokic et al. (2005b).

Para resumir, la tabla 2.4 recopila las tensiones residuales mínimas, $V_{\text {res }}$ min , que se han obtenido en base a la extensa revisión bibliográfica realizada, en función de la potencia nominal, $P_{n}$, del variador de frecuencia. De esta manera, $V_{\text {res }}$ min implica que cualquier hueco de tensión que posea una tensión residual por encima de dicho valor no causará la desconexión del variador de frecuencia. Además, se indica el tiempo máximo, $t_{\max }$, que soporta el variador de frecuencia para una interrupción total del suministro. Así, como se ha visto que el comportamiento del variador de frecuencia responde a una curva de tolerancia perfectamente rectangular, la tabla 2.4 representa de manera precisa la sensibilidad de este tipo de aparamenta, dado que realmente el par de valores mostrados en la tabla, $V_{r e s_{\min }}$ y $t_{\max }$, representan la esquina superior izquierda de la curva de tolerancia.

Sobre la tabla 2.4 se pueden obtener algunas conclusiones generales. Puede verse que este tipo de aparamenta tiene una sensibilidad muy elevada ante huecos de tensión dado que la mayoría de variadores de frecuencia disparan 


\begin{tabular}{|c|c|c|c|}
\hline Referencia & $P_{n}$ & $V_{r e s_{\min }}$ & $t_{\max }$ \\
\hline Sarmiento y Estrada (1996) & - & $80 \%$ & $200 \mathrm{~ms}$ \\
\hline Keus et al. $(1999)$ & $15 \mathrm{~kW}$ & $80-90 \%$ & $20-50 \mathrm{~ms}$ \\
\hline \begin{tabular}{|l|l} 
Stockman et al. & $(2003)$, baja capacidad \\
Stockman et al. & $(2003)$, alta capacidad \\
\end{tabular} & - & $\begin{array}{l}90 \% \\
78 \% \\
\end{array}$ & $\begin{array}{c}3-15 \mathrm{~ms} \\
18-48 \mathrm{~ms}\end{array}$ \\
\hline 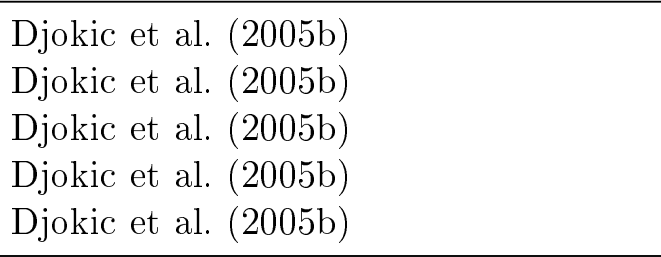 & $\begin{array}{c}4 \mathrm{~kW} \\
4 \mathrm{~kW} \\
3.7 \mathrm{~kW} \\
4 \mathrm{~kW} \\
4 \mathrm{~kW}\end{array}$ & $\begin{array}{l}73 \% \\
76 \% \\
75 \% \\
90 \% \\
88 \% \\
\end{array}$ & $\begin{array}{c}18 \mathrm{~ms} \\
22 \mathrm{~ms} \\
20 \mathrm{~ms} \\
5 \mathrm{~ms} \\
10 \mathrm{~ms}\end{array}$ \\
\hline Vegunta et al. (2006) & - & $30 \%$ & $10 \mathrm{~ms}$ \\
\hline \begin{tabular}{|l|l} 
Djokic et al. $(2008)$ \\
\end{tabular} & - & $85 \%$ & $10 \mathrm{~ms}$ \\
\hline
\end{tabular}

Tabla 2.4: Resumen de los principales estudios realizados sobre huecos de tensión en variadores de frecuencia.

frente a huecos de tensión con profundidades de hueco superiores al $20 \%$. Además, el tiempo que pueden soportar una interrupción en algunos casos es inferior a 1 ciclo. También se observa un resultado que difiere en gran medida con respecto a los anteriores. Concretamente, Vegunta et al. (2006) simula bajo el software PSCAD/EMTDC un variador de frecuencia con sistema regenerativo, permitiendo aumentar considerablemente el rango de tensiones admisibles de alimentación del variador. Otra puntualización importante a tener en cuenta es que algunos autores exponen los resultados sin dar información sobre algunas características básicas del variador de frecuencia, como su potencia nominal.

Tal y como se ha ido comentando a lo largo del presente apartado, la línea horizontal de la curva de tolerancia del variador de frecuencia - caracterizada

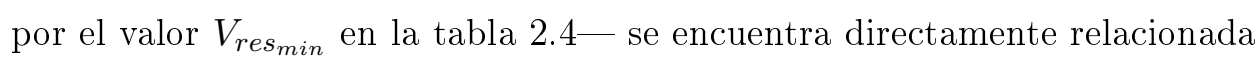
con la cantidad de energía que puede atravesar el variador, es decir, con el valor máximo de intensidad admisible por el mismo. Además, se encuentra condicionada por la tensión mínima aceptable del bus de continua. De hecho, esta línea horizontal es siempre superior al valor mínimo de la tensión admisible en el bus de continua, debido al rizado en dicho bus. Sin embargo, la línea vertical de la curva de tolerancia está directamente relacionada con la energía almacenada en la etapa de continua.

Los ensayos realizados con el variador de frecuencia en la presente Tesis Doctoral, mostrados en el apartado 2.4.3, emplean uno de los tipos de variadores más utilizados en Baja Tensión, que es el que posee alimentación 
monofásica y tensión trifásica de salida. Como se puede comprobar en la revisión bibliográfica realizada, no se conocen ensayos realizados sobre este tipo de variadores de frecuencia. Al contrario que los variadores de frecuencia monofásicos, sólo el $3 \%$ de los motores conectados en Media Tensión son controlados por variadores de frecuencia, Abu-Rub et al. (2010). De hecho, debido a que no se suelen encontrar estos equipos funcionando a elevadas tensiones de alimentación, en Endrejat et al. (2008) se detalla el proceso de conexión de un variador de frecuencia de $15.5 \mathrm{MW}$ y $11 \mathrm{kV}$ de potencia y tensión nominal, respectivamente, en una industria petroquímica.

Además, dada la influencia de los diversos regímenes de carga en la respuesta de este tipo de aparamenta, se ha evaluado el comportamiento del equipo en función de su frecuencia de salida.

\subsubsection{Análisis del ordenador personal}

Los elementos pertenecientes a las tecnologías de la información, entre los que se encuentra el ordenador personal — nombrado de aquí en adelante como "Personal Computer (PC)"-, son ampliamente utilizados para control de procesos; por lo que resulta interesante comprender qué tipos de perturbaciones van a modificar su funcionamiento y los efectos asociados a las mismas.

Primeramente, es importante tener en cuenta que los microprocesadores también son altamente sensibles a los huecos de tensión, Pohjanheimo y Lehtonen (2002); Barros y Diego (2002); Saksena y Karady (2005); Djokic et al. (2005a); Baggini (2008); Shareef et al. (2009a); Hardi y Daut (2010); Akolkar y Kushare (2010). Los ordenadores personales aparecieron en la década de los 70 y hoy en día este tipo de aparamenta eléctrica está compuesta por fuentes de alimentación, conocidas como fuentes conmutadas —o "switch mode power supply"-, que contienen una etapa rectificadora junto con una etapa de continua, permitiendo así la alimentación del resto de componentes electrónicos. La etapa rectificadora básicamente está constituida por un puente de diodos conectado a un condensador a su salida con el objeto de conseguir una tensión constante. Posteriormente, se conecta un convertidor de corriente continua que posibilita la conmutación a alta frecuencia, tal y como muestra la figura 2.11. Adicionalmente puede contener también elementos de protección contra cortocircuitos y/o variaciones de tensión.

Por lo tanto, de manera similar a lo que ocurría en los variadores de frecuencia analizados en el apartado 2.2 .3 , la capacidad de soportar huecos de tensión de una fuente de alimentación se encuentra directamente relacionada

con la energía almacenada en sus condensadores, Brauner y Hennerbichler (2001); Djokic et al. (2005a). De esta manera, si la tensión de alimentación se reduce, la tensión en el bus de continua también decrece y, por lo tanto, se puede producir el fallo de los componentes electrónicos del equipo, ocasionando varios tipos de problemas, Baran et al. (1998); Djokic et al. (2005a); 


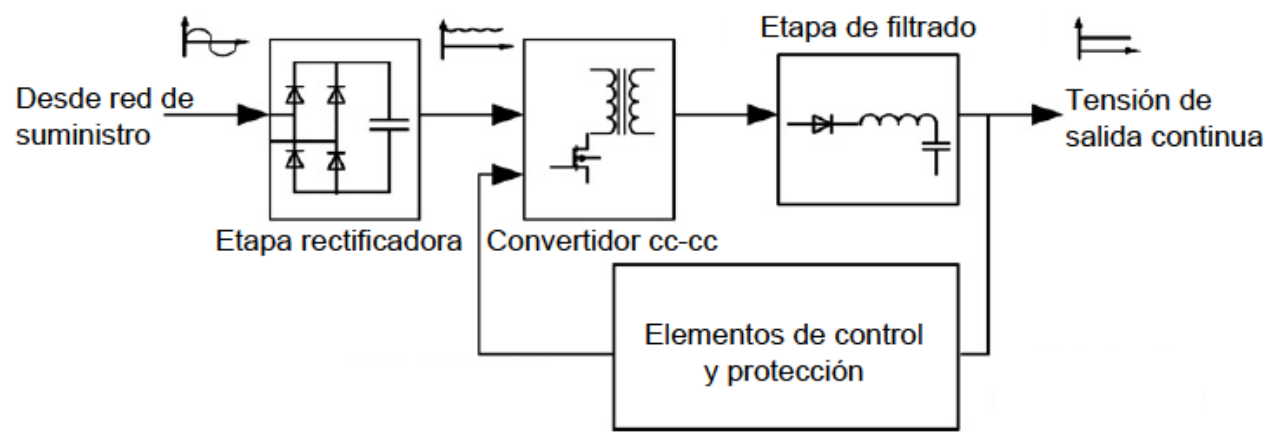

Figura 2.11: Esquema constructivo de una fuente de alimentación conmutada.

Bok et al. 2008); Chilukuri et al. (2009):

- Pérdida de información.

- Pérdida de control sobre los periféricos del equipo.

- Bloqueo del sistema operativo.

- Reinicio completo del $\mathrm{PC}$

- Destrucción de la fuente de alimentación.

Concretamente, la ecuación que gobierna el tiempo, $T_{H}$, que puede soportar una fuente conmutada una interrupción del suministro se encuentra determinada por Fernandez et al. (2005); Diaz et al. (2007); Shareef et al. (2010b):

$$
T_{H}=\frac{C \cdot\left(V_{n o m}^{2}-V_{m i n}^{2}\right)}{2 \cdot P}
$$

Siendo $V_{\text {nom }}$ la tensión nominal de continua, $V_{\min }$ la tensión mínima aceptable por el condensador para que el convertidor de continua actúe correctamente - según Fernandez et al. (2005), $V_{\min }$ suele valer tres veces menos que $V_{\text {nom }}$ - $P$ es la potencia nominal de la fuente conmutada, y $C$ es la capacidad del condensador situado a la salida del puente de diodos. De esta manera, queda comprendido que el aumento de la capacidad del condensador aumenta el tiempo que el equipo puede soportar ante una pérdida total de la tensión. Sin embargo, por un lado, la ecuación (2.2) sólo hace referencia a la variable tiempo $y$, por otro, la elevada capacidad necesaria para soportar huecos de tensión de larga duración resulta ser inviable, Shareef et al. (2009a). Además, el aumento de la capacidad ocasiona que, en el momento en que se restaure la tensión de alimentación, aumente el pico de 
intensidad absorbida. Por otro lado, en este tipo de cargas resulta sencillo la mejora de su respuesta frente a huecos de tensión mediante la conexión de un Sistema de Alimentación Ininterrumpida (SAI) - también conocido como "Uninterruptible Power Supply (UPS)"-, Chilukuri et al. (2009).

Al igual que ocurría con los variadores de frecuencia, y contrariamente al caso de los contactores, el ángulo de inicio de la onda de tensión no afecta al comportamiento de estos equipos frente a huecos de tensión, Saksena y Karady (2005); Djokic et al. (2005a); Hardi y Daut (2010). Además, en base a los trabajos publicados por diferentes autores, la curva de tolerancia de un PC describe una forma prácticamente rectangular, de manera similar a lo investigado en los variadores de frecuencia. Continuando en la misma línea, aunque la respuesta de este tipo de cargas pueda parecer sencilla, existen varios trabajos de investigación publicados por diversos autores.

El primer estudio que se encuentra en la bibliografía, Rob et al. (1993), realiza solamente seis ensayos sobre un $\mathrm{PC}$ observando que el reinicio del mismo se produce para profundidades del hueco de tensión superiores al $22 \%$. Además, indica que ante una interrupción del suministro de $50 \mathrm{~ms}$ también se produce el reinicio del $\mathrm{PC}$.

En otro de los primeros análisis, Brauner y Hennerbichler (2001), se ensayan varios ordenadores personales, aunque sin aportar información sobre las características técnicas de los mismos. Dicho estudio muestra que el mejor PC ensayado puede soportar huecos de tensión de hasta el $68 \%$ de profundidad de manera continua, así como interrupciones del suministro de $450 \mathrm{~ms}$; mientras que el $\mathrm{PC}$ más sensible soportaría una profundidad del $35 \%$ y una duración de 80 ms. Con el mismo objetivo, Pohjanheimo y Lehtonen (2002) ensaya siete ordenadores personales de diferente fabricante y año fabricación, aunque concluye que no existe una relación evidente entre el año de fabricación del $[\mathrm{PC}$ y su respuesta frente a huecos de tensión. Esta misma conclusión se mantiene en Saksena y Karady (2005); Djokic et al. (2005a). En la misma época que Pohjanheimo y Lehtonen (2002), Barros y Diego (2002) publica su estudio, donde obtiene la curva de tolerancia de un solo $\mathrm{PC}$, en función del contenido de armónicos de la red que alimenta al equipo.

De manera similar, Saksena y Karady (2005) ensaya dos ordenadores de diferentes procesadores (Pentium II frente a Pentium III), señalando que el comportamiento es idéntico. Afirma que si la profundidad del hueco es superior al $30 \%$ y la duración es mayor que $130 \mathrm{~ms}$ se produce el reinicio del PC. Una aportación interesante de Saksena y Karady (2005) es que señala que si el consumo del $\mathrm{PC}$ se eleva - debido, por ejemplo, a una operación de copiado de información desde el lector de cd al disco duro-, se perjudica su respuesta frente a huecos de tensión. Como se puede observar, esta afirmación es coherente con el comportamiento del variador de frecuencia en función del régimen de carga, mostrado en el apartado 2.2 .3 .

En Djokic et al. (2005a) se presenta uno de los más extensos trabajos rea- 
lizados hasta la fecha en esta temática. Ensaya seis ordenadores personales, definiendo todas sus características. Esta información adicional permite que se pueda concluir que el componente del $\mathrm{PC}$ que más influencia tiene sobre la respuesta del mismo ante huecos de tensión es la fuente de alimentación del mismo. Además, obtiene diferentes curvas de tolerancia para cada $\mathrm{PC}$ en función del tipo de problema que pretende detectar (pérdida de información, bloqueo del sistema operativo, o reinicio del (PC), coincidiendo en conclusiones con Saksena y Karady (2005); Bok et al. (2008). Esta circunstancia podría ocasionar que el mismo $\mathrm{PC}$ se encuentre dentro de los límites de una curva de tolerancia, ya sea ITI o SEMI, cuando se evalúe el reinicio del $\mathrm{PC}$, mientras que podría no cumplir dichas curvas si se evalúa la capacidad de bloqueo del sistema operativo. Otra consideración importante es la elevada intensidad que absorbe el $[\mathrm{PC}$ cuando se recupera la tensión nominal después de un hueco de tensión que no cause el reinicio del equipo, encontrándose valores desde 9 hasta 25 veces superior a la intensidad nominal, Djokic et al. (2005b); Chilukuri et al. (2009); Hardi y Daut (2010). Por último, Djokic et al. (2005b) también señala que la respuesta del $\overline{\mathrm{PC}}$ resulta modificada en función del contenido de armónicos de la red de suministro, en línea con lo publicado por Barros y Diego (2002).

Como anécdota, resulta interesante comentar que el trabajo presentado en Djokic et al. (2005a) fue discutido por los autores que realizaron Barros y Diego (2002). En Barros y Diego (2006); Djokic y Milanovic (2006) se encuentra el debate que mantuvieron donde, básicamente, el primero de los trabajos (de manera cronológica) critica al más reciente porque no le ha tenido en cuenta en la revisión bibliográfica, además de señalarle que el contenido de armónicos de la red de suministro que alimenta al $\mathrm{PC}$ es demasiado alto y se aleja de una situación real.

Más tarde, en Bok et al. (2008) se obtienen las curvas de tolerancia de ocho ordenadores personales con diferentes fuentes de alimentación. Además de coincidir con el resto de autores citados anteriormente, en función de los resultados de sus ensayos se puede deducir que las fuentes de alimentación que posean componentes activos para la corrección del factor de potencia tienen mejor comportamiento ante huecos de tensión que las que sólo poseen elementos pasivos.

Otro de los trabajos con mayor desarrollo experimental que se conoce es Shareef et al. (2009a), donde cinco ordenadores personales correctamente especificados son ensayados frente a huecos de tensión, observando que si la tensión residual del hueco de tensión no se reduce más del $50 \%$ no se produce el disparo de ningún PC] Además, Shareef et al. (2009a) muestra experimentalmente las ventajas y desventajas del aumento de la capacidad conectada después de la etapa rectificadora sobre la respuesta de estos equipos ante huecos de tensión.

En Chilukuri et al. (2009) se presenta un trabajo sobre efectos de huecos 
de tensión en diversos equipos domésticos, aunque la precisión de sus ensayos es relativamente reducida. Entre ellos, en el ensayo de dos $\mathrm{PC}$ le permite concluir que el $\mathrm{PC}$ más moderno tiene una mejor respuesta ante huecos de tensión. Esta conclusión también la mantiene Hardi y Daut (2010), después del ensayo de tres $\overline{\mathrm{PC}}$ de diferente año de construcción.

Con el objeto de identificar de una manera sencilla los diversos resultados obtenidos por otros autores en relación con el comportamiento de $\mathrm{PC}$; ante huecos de tensión, la tabla 2.5 muestra las tensiones residuales mínimas, $V_{\text {res }_{\min }}$, que soportan estos equipos para prevenir el reinicio de los mismos. Igualmente, se indica el tiempo máximo $t_{\max }$ que puede soportar el $\mathrm{PC}$ una interrupción total del suministro. Se ha decidido exponer estos dos parámetros característicos en función de la tensión y potencia nominales, $V_{n}$ y $P_{n}$, de la fuente de alimentación asociada a cada PC. Así, como ha quedado justificado que el comportamiento del $\mathrm{PC}$ responde a una curva de tolerancia rectangular, la tabla 2.5 es una precisa representación de la sensibilidad de este tipo de equipamiento.

La primera anotación sobre la tabla 2.5 muestra que en los datos deducidos del trabajo de Bok et al. (2008) se ha incluido la información del tipo de fuente de alimentación en lugar de su tensión nominal, puesto que indica que las fuentes de alimentación con corrección del factor de potencia con elementos activos (denotado como "act $P F C$ ") poseen mejor respuesta ante huecos de tensión que las que están constituidas solamente por elementos pasivos ("pas PFC"). Seguidamente, se puede observar que existe una gran diversidad de resultados; dado que en relación con el tiempo que puede soportar esta aparamenta una interrupción del suministro, el mejor equipo es capaz de aguantar $500 \mathrm{~ms}$, mientras que el $\mathrm{PC}$ más sensible soporta sólo $42 \mathrm{~ms}$. En cuanto a la capacidad por soportar huecos de tensión de manera prolongada, también existe una amplia diversidad, ya que hay ensayos en los que no dispara el $\mathrm{PC}$ hasta que la profundidad del mismo es superior al $85 \%$; mientras que en otros equipos ensayados, con una profundidad tan solo del $22 \%$ se produce el reinicio los mismos. La información expuesta en la tabla 2.5 permite apreciar que en los estudios más recientes existe una mejor respuesta del $\mathrm{PC}$ frente a las perturbaciones de red. De todas maneras, se puede observar cómo, en bastantes de los trabajos revisados, no se da información acerca del $\mathrm{PC}$ ensayado ni de la fuente de alimentación asociada al mismo.

En cuanto al grado de cumplimiento de este tipo de aparamenta eléctrica con respecto a las curvas ITI y SEMI, se puede afirmar que el $94 \%$ de los ensayos encontrados en la bibliografía se encuentran dentro del contorno definido por la Norma ITI. Sin embargo, en el caso de la Norma SEMI el porcentaje de $\mathrm{PC}$ ensayados que la cumplen se reduce al $64 \%$. Precisamente, la severidad inherente a la curva SEMI en la región comprendida entre $20 \mathrm{~ms}$ y $200 \mathrm{~ms}$ es la causante de estas afirmaciones. 


\begin{tabular}{|c|c|c|c|c|}
\hline Referencia & $\overline{V_{n}}$ & $P_{n}$ & $\overline{V_{\text {res } \min }}$ & $t_{\max }$ \\
\hline Rob et al. (1993) & - & - & $78 \%$ & $50 \mathrm{~ms}$ \\
\hline \begin{tabular}{|l|l} 
Brauner y Hennerbichler \\
Brauner y Hennerbichler
\end{tabular}$\left(\frac{2001}{2001}\right)$ & - & - & $\begin{array}{l}65 \% \\
32 \%\end{array}$ & $\begin{array}{l}80 \mathrm{~ms} \\
450 \mathrm{~ms}\end{array}$ \\
\hline \begin{tabular}{|l|l|} 
Barros y Diego $(2002)$ \\
\end{tabular} & - & - & $49 \%$ & $386 \mathrm{~ms}$ \\
\hline 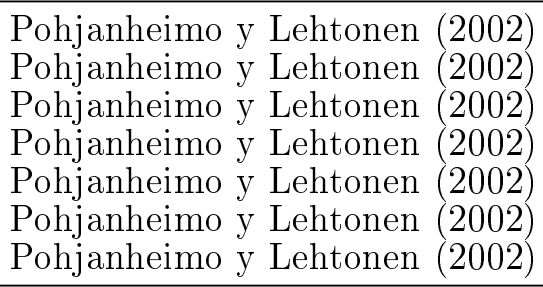 & $\begin{array}{l}- \\
- \\
- \\
-\end{array}$ & $\begin{array}{l}E \\
E \\
E \\
- \\
-\end{array}$ & $\begin{array}{l}72 \% \\
62 \% \\
55 \% \\
58 \% \\
38 \% \\
58 \% \\
53 \% \\
\end{array}$ & $\begin{array}{l}50 \mathrm{~ms} \\
120 \mathrm{~ms} \\
140 \mathrm{~ms} \\
150 \mathrm{~ms} \\
225 \mathrm{~ms} \\
260 \mathrm{~ms} \\
280 \mathrm{~ms}\end{array}$ \\
\hline Saksena y Karady (2005) & - & - & $70 \%$ & $130 \mathrm{~ms}$ \\
\hline Djokic et al. (2005a) & $\begin{array}{l}110-120 \mathrm{~V} \\
200-240 \mathrm{~V}\end{array}$ & $235 \mathrm{~W}$ & $21 \%$ & $380 \mathrm{~ms}$ \\
\hline$\frac{\text { Djokic et al. }}{\text { Djokic et al. }}\left(\frac{2005 \mathrm{a}}{(2005 \mathrm{a}}\right)$ & $\begin{array}{l}115-230 \mathrm{~V} \\
115-230 \mathrm{~V}\end{array}$ & $\begin{array}{l}300 \mathrm{~W} \\
300 \mathrm{~W}\end{array}$ & $\begin{array}{l}63 \% \\
62 \%\end{array}$ & $\begin{array}{l}42 \mathrm{~ms} \\
45 \mathrm{~ms}\end{array}$ \\
\hline Djokic et al. (2005a) & $\begin{array}{l}110-120 \mathrm{~V} \\
200-240 \mathrm{~V}\end{array}$ & $235 \mathrm{~W}$ & $58 \%$ & $170 \mathrm{~ms}$ \\
\hline 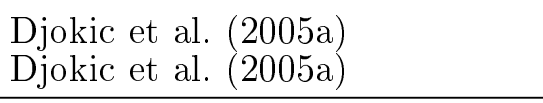 & $\begin{array}{l}115-230 \mathrm{~V} \\
200-240 \mathrm{~V}\end{array}$ & $\begin{array}{l}250 \mathrm{~W} \\
200 \mathrm{~W}\end{array}$ & $\begin{array}{l}60 \% \\
50 \% \\
\end{array}$ & $\begin{array}{l}120 \mathrm{~ms} \\
115 \mathrm{~ms}\end{array}$ \\
\hline Moon et al. (2007) & - & - & $62 \%$ & $70 \mathrm{~ms}$ \\
\hline 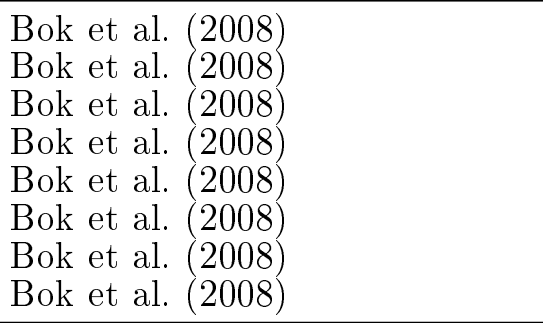 & $\begin{array}{l}\text { act } \mathrm{PFC} \\
\text { act } \mathrm{PFC} \\
\text { pas PFC } \\
\text { pas PFC } \\
\text { pas PFC } \\
\text { pas PFC } \\
\text { pas PFC } \\
\text { pas PFC }\end{array}$ & $\begin{array}{l}300 \mathrm{~W} \\
300 \mathrm{~W} \\
300 \mathrm{~W} \\
250 \mathrm{~W} \\
200 \mathrm{~W} \\
235 \mathrm{~W} \\
200 \mathrm{~W} \\
250 \mathrm{~W}\end{array}$ & $\begin{array}{l}18 \% \\
19 \% \\
42 \% \\
50 \% \\
58 \% \\
61 \% \\
65 \% \\
50 \% \\
\end{array}$ & $\begin{array}{l}280 \mathrm{~ms} \\
180 \mathrm{~ms} \\
190 \mathrm{~ms} \\
200 \mathrm{~ms} \\
130 \mathrm{~ms} \\
140 \mathrm{~ms} \\
120 \mathrm{~ms} \\
100 \mathrm{~ms}\end{array}$ \\
\hline Shareef et al. (2009a) & $\begin{array}{l}100-120 \mathrm{~V} \\
200-240 \mathrm{~V}\end{array}$ & $180 \mathrm{~W}$ & $25 \%$ & $160 \mathrm{~ms}$ \\
\hline Shareef et al. (2009a) & $\begin{array}{l}100-127 \mathrm{~V} \\
200-240 \mathrm{~V}\end{array}$ & $250 \mathrm{~W}$ & $40 \%$ & $240 \mathrm{~ms}$ \\
\hline Shareef et al. (2009a) & $\begin{array}{l}100-127 \mathrm{~V} \\
200-240 \mathrm{~V}\end{array}$ & $250 \mathrm{~W}$ & $46 \%$ & $280 \mathrm{~ms}$ \\
\hline Shareef et al. (2009a) & $\begin{array}{l}100-127 \mathrm{~V} \\
200-240 \mathrm{~V}\end{array}$ & $300 \mathrm{~W}$ & $50 \%$ & $220 \mathrm{~ms}$ \\
\hline \begin{tabular}{|l|l} 
Chilukuri et al. & $\left(\frac{2009}{2009}\right)$ \\
Chilukuri et al. & $\left(\frac{2009}{2}\right)$
\end{tabular} & - & - & $\begin{array}{l}15 \% \\
40 \%\end{array}$ & $\begin{array}{l}400 \mathrm{~ms} \\
200 \mathrm{~ms}\end{array}$ \\
\hline \begin{tabular}{l|l} 
Hardi y Daut \\
Hardi y Daut \\
Hardi y Daut
\end{tabular}$\left(\begin{array}{l}\frac{2010}{2010} \\
\left(\frac{2010}{201}\right)\end{array}\right.$ & - & - & $\begin{array}{l}40 \% \\
50 \% \\
50 \%\end{array}$ & $\begin{array}{l}500 \mathrm{~ms} \\
200 \mathrm{~ms} \\
100 \mathrm{~ms}\end{array}$ \\
\hline
\end{tabular}

Tabla 2.5: Resumen de los principales estudios realizados sobre huecos de tensión en ordenadores personales. 


\subsubsection{Análisis de diversas cargas de iluminación}

Hoy en día, el consumo de energía eléctrica en forma de iluminación representa en torno al $20 \%$ del consumo eléctrico a nivel mundial, Honrubia Escribano et al. (2012b). Dentro del abanico de posibilidades existente en este sector, los tipos tecnologías más empleadas son las siguientes:

- Lámparas incandescentes: este tipo de iluminación ha permanecido prácticamente invariable en la historia desde su aparición en el siglo XIX, Frater y Watson (2007). Básicamente, estas lámparas están compuestas por un filamento de tungsteno dentro de una cavidad de vidrio llenada por un gas inerte. De esta manera, el paso de corriente eléctrica por dicho filamento provoca el calentamiento del mismo hasta una temperatura de $2000 \mathrm{~K}-3000 \mathrm{~K}$, emitiendo luz visible. La alta temperatura alcanzada por el filamento es la causante del reducido rendimiento, así como de la limitada vida útil, de este tipo de lámparas.

- Lámparas de descarga: ampliamente utilizadas para la iluminación de grandes áreas, Pohjanheimo y Lehtonen (2002); Diaz et al. (2007), esta tecnología se subdivide en varios tipos de lámparas en función de la presión del gas que contienen. Así, se encuentran lámparas fluorescentes lineales y fluorescentes compactas que contienen vapor de mercurio a baja presión, lámparas de vapor de sodio tanto a baja como a alta presión, lámparas de halogenuros metálicos, etc. En esencia, su característica principal es que este tipo de tecnología necesita un transitorio de alta tensión sobre los terminales de la lámpara para iniciar el proceso de descarga del gas en el interior del recipiente que lo contiene.

- Diodos emisores de luz, comúnmente conocidos como "Light Emiting Diode (LED)": se trata de una tecnología que, aunque bastante antigua, cada día va ocupando una mayor posición en el mercado.

En importante mencionar la situación actual relacionada con la eficiencia energética de las tecnologías listadas anteriormente. La Directiva 2009/125/CE, conocida como de ecodiseño, sienta las bases para el establecimiento de requisitos de diseño ecológico aplicables a los productos relacionados con la energía. Esta Directiva, que es la actualización de la Directiva 2005/32/CE —conocida como EuP ("Energy-using Products"), Philips (2010)—, persigue la desaparición de aquellas tecnologías de iluminación poco eficientes. En el caso de la iluminación incandescente, en el año 2009 se inició la prohibición de fabricación de este tipo de lámparas de manera progresiva en función de su potencia nominal; causando que en septiembre de 2012 se prohibiese completamente la fabricación de estas lámparas en la Unión Europea. Igualmente, se prohíbe la fabricación de determinadas lámparas fluorescentes.

La iluminación fluorescente es el sistema de iluminación más extendido a nivel mundial, Shareef et al. (2009b); Hsieh y Lin (2011), encontrándose 
en una diversidad de aplicaciones, como supermercados, oficinas, parkings, aeropuertos, etc. Particularmente, en el caso de la energía eólica, es muy común encontrar este tipo de iluminación en cualquier centro de control de un parque eólico. Dentro de este tipo de iluminación existen dos tecnologías claramente diferenciadas. La primera y más antigua tecnología, conocida como fluorescente convencional, está constituida por un cebador y un balasto electromagnético, teniendo presencia en el $75 \%$ de las oficinas de Europa, ELCF (2007). Por otro lado, existe la tecnología electrónica que, suprimiendo el cebador y la reactancia electromagnética por un balasto electrónico — se trata de una fuente de alimentación conmutada-, proporciona una tensión de alta frecuencia sobre los terminales de la lámpara. Este sistema electrónico posee una serie de ventajas sobre el tradicional, Diaz et al. (2007); Shareef et al. (2010b); Honrubia Escribano et al. (2012b): reducción de volumen así como de peso, alto factor de potencia, alto rendimiento, posibilidad de regular el nivel de iluminación en función de las condiciones ambientales, supresión del efecto estroboscópico, aumento de la vida de la lámpara y la ausencia de ruido acústico, entre otros.

Dada la relevancia que este tipo de cargas tienen sobre el sistema eléctrico, resulta necesario un estudio de su comportamiento ante perturbaciones en la red. La variación del flujo luminoso -fenómeno conocido como "flicker"es el efecto principal de los huecos de tensión sobre este tipo de aparamenta eléctrica aunque, en función de los parámetros asociados al hueco de tensión y el tipo de lámpara, se podría incluso conseguir la desconexión de la misma. Dicha desconexión involuntaria puede ocasionar inconvenientes debido a que algunas lámparas no permiten un reencendido instantáneo. Concretamente, en lámparas de vapor de sodio a alta presión — ampliamente utilizadas en la iluminación de viales-, debido al proceso de enfriamiento de la lámpara, son necesarios varios minutos hasta que se pueda conseguir el reencendido de la lámpara después de una desconexión de la misma, Dorr et al. (1997a); Diaz et al. (2007); Menniti et al. (2010). En otras lámparas de descarga, este tiempo puede incrementarse hasta 15 minutos, Brauner y Hennerbichler (2001).

Uno de los primeros trabajos desarrollados en esta temática se encuentra en Dorr et al. (1997a), donde ensaya tres lámparas de vapor de sodio a alta presión. Sus resultados muestran que este tipo de lámparas puede soportar huecos de tensión con una tensión residual entre el $62 \%$ y el $71 \%$ de manera ininterrumpida; mientras que una interrupción total del suministro la pueden soportar $17 \mathrm{~ms}$. Además, Dorr et al. (1997a) observa que el envejecimiento de este tipo de lámparas origina un aumento de su sensibilidad ante huecos de tensión. En Brauner y Hennerbichler (2001) se obtiene una curva de tolerancia similar, prácticamente rectangular, ensayando una lámpara de vapor de mercurio a alta presión. 
En Pohjanheimo y Lehtonen (2002) se ensayan siete lámparas de descarga de diferentes tecnologías frente a huecos de tensión, volviendo a poner de manifiesto la elevada sensibilidad de este tipo de cargas. De hecho, la rectangular curva de tolerancia obtenida muestra que ninguna de las lámparas empleadas soporta una interrupción del suministro superior a $25 \mathrm{~ms}$. Una aportación adicional es que observan que cuanto más moderna es la lámpara ensayada, mejor es su respuesta frente a perturbaciones de red.

El trabajo presentado en Diaz et al. (2007) compara la respuesta del balasto electromagnético con el electrónico para una lámpara de vapor de sodio a alta presión. Muestra que el balasto electrónico confiere un mejor comportamiento ante huecos de tensión e interrupciones, afirmación posteriormente confirmada por Drapela et al. (2009). Continuando con la misma tendencia, Shareef et al. (2009b) compara la respuesta de dos lámparas fluorescentes lineales de $18 \mathrm{~W}$ de potencia nominal, una alimentada con balasto convencional y otra con balasto electrónico, obteniendo la misma conclusión. Concretamente, el ensayo presentado en Diaz et al. (2007) con dos balastos electrónicos muestra que la lámpara puede soportar $20 \mathrm{~ms}$ sin apagarse; mientras que el electromagnético no llega a soportar 10 ms. Además, Diaz et al. (2007) analiza cómo, en el caso de la lámpara alimentada por el balasto electromagnético, se produce el aumento de la sensibilidad ante huecos de tensión directamente proporcional al envejecimiento de la lámpara, en línea con Dorr et al. (1997a); Pohjanheimo y Lehtonen (2002).

En Drapela et al. (2009) se presenta uno de los análisis más extensos realizados hasta la fecha en este ámbito. Obtiene la curva de tolerancia de trece lámparas de diferentes tecnologías. Sus resultados muestran que la iluminación fluorescente con balasto electrónico posee la mayor tolerancia frente a huecos de tensión e interrupciones; mientras que los valores más críticos se obtienen en lámparas de descarga de alta presión alimentadas por balastos electromagnéticos.

Shareef et al. (2010b) se centra en el comportamiento de las lámparas fluorescentes compactas ante huecos de tensión e interrupciones. Después de ensayar seis lámparas de esta tecnología controladas por balastos electrónicos obtiene principalmente dos conclusiones. Por un lado, observa que la lámpara que posee la peor respuesta ante interrupciones es la que menor capacidad posee en su etapa de continua. Además, afirma que la lámpara de menor potencia nominal soporta una tensión residual menor de manera ininterrumpida que la lámpara de mayor potencia nominal del mismo fabricante. Sin embargo, en su trabajo se puede observar que la afirmación anterior pierde validez si se comparan resultados entre diferentes fabricantes.

Los ensayos presentados en Hardi y Daut (2010) sobre dos lámparas fluorescentes compactas (una equipada con balasto electrónico y otra con balasto electromagnético) obtienen resultados similares. Aunque, en este caso, la lámpara conectada con balasto electromagnético soporta menor tensión re- 
sidual que el balasto electrónico, al contrario que los ensayos realizados en Diaz et al. (2007); Shareef et al. (2009b); Drapela et al. (2009). No obstante, la conclusión de Hardi y Daut (2010) está basada en dos lámparas de diferente potencia nominal.

$\mathrm{Al}$ igual que se viene haciendo con el resto de aparamenta eléctrica analizada, la tabla 2.6 muestra las tensiones residuales mínimas, $V_{r e s_{\text {min }}}$, que soportan estos equipos de manera ininterrumpida sin producirse su desconexión, junto con el tiempo máximo, $t_{\max }$, que pueden soportar una interrupción del suministro. Estos dos parámetros característicos se han expuesto en función del tipo de tecnología luminosa y su potencia nominal, $P_{n}$, puesto que estas dos propiedades han resultado ser los elementos más influyentes en la respuesta de esta aparamenta eléctrica frente a perturbaciones en la red eléctrica. Los acrónimos que aparecen en dicha tabla representan las siguientes tecnologías: Vapor de Sodio a Alta Presión (VSAP), Vapor de Mercurio a Alta Presión (VSAP), Halogenuros Metálicos ( $(\mathrm{HM})$, Lámpara Fluorescente Lineal (LFL), Lámpara Fluorescente Compacta (CFL). En algunas tecnologías aparece la letra "e" delante del acrónimo para señalar que se emplea un balasto electrónico.

En vista de la tabla 2.6, se puede observar que el cumplimiento de las curvas ITI y SEMI se encuentra bastante comprometido. De hecho, sólo el $40 \%$ y el $13 \%$ de las diferentes cargas de iluminación presentes en dicha tabla se encuentran dentro de los contornos definidos por dichas curvas, respectivamente. Debido a esta situación, se está en condiciones de considerar que este tipo de equipamiento es muy sensible a las interrupciones del suministro eléctrico.

Una vez analizados los estudios publicados anteriormente, es importante tener en cuenta que en los experimentos practicados en la presente Tesis Doctoral sobre diversas cargas de iluminación se han tenido en cuenta las siguientes tecnologías:

- Iluminación fluorescente: dado que esta tecnología es la más extendida a nivel mundial, se han realizado ensayos sobre tubos fluorescentes alimentados tanto por balasto electromagnético como por balasto electrónico.

- Lámparas de inducción: esta tecnología, aunque su coste representa una de sus mayores desventajas, posee un rendimiento muy elevado. Además, en la revisión bibliográfica realizada no se ha encontrado ningún estudio que obtenga su curva de tolerancia.

- Lámparas de halogenuros metálicos: debido a su elevada utilización para la iluminación de grandes áreas y a la reducida información encontrada en la bibliografía con respecto a su comportamiento frente a perturbaciones de red, también se han ensayado. 


\begin{tabular}{|c|c|c|c|c|}
\hline Referencia & $\begin{array}{l}\text { Tipo de } \\
\text { lámpara }\end{array}$ & $P_{n}$ & $V_{\text {res }}$ min & $t_{\max }$ \\
\hline 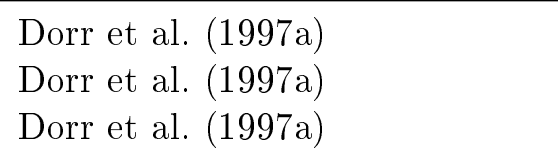 & $\begin{array}{l}\text { VSAP } \\
\text { VSAP } \\
\text { VSAP }\end{array}$ & $\begin{array}{l}400 \mathrm{~W} \\
400 \mathrm{~W} \\
400 \mathrm{~W}\end{array}$ & $\begin{array}{l}62 \% \\
71 \% \\
72 \%\end{array}$ & $\begin{array}{l}17 \mathrm{~ms} \\
8 \mathrm{~ms} \\
8 \mathrm{~ms}\end{array}$ \\
\hline Brauner y Hennerbichler (2001) & VMAP & - & $79 \%$ & $10 \mathrm{~ms}$ \\
\hline 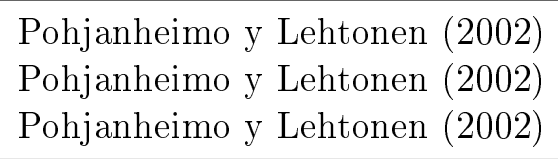 & $\begin{array}{c}\text { HM } \\
\text { VSAP } \\
\text { VMAP }\end{array}$ & $\begin{array}{l}250 \mathrm{~W} \\
250 \mathrm{~W} \\
80 \mathrm{~W}\end{array}$ & $\begin{array}{l}50 \% \\
55 \% \\
80 \%\end{array}$ & $\begin{array}{l}- \\
25 \mathrm{~ms} \\
10 \mathrm{~ms}\end{array}$ \\
\hline \begin{tabular}{|l|l|} 
Diaz et al. & $\left(\frac{2007}{207}\right)$ \\
Diaz et al. & $\left(\frac{2007}{20}\right)$ \\
Diaz et al. & $(2007)$ \\
\end{tabular} & $\begin{array}{l}\text { eVSAP } \\
\text { eVSAP } \\
\text { VSAP }\end{array}$ & $\begin{array}{l}150 \mathrm{~W} \\
150 \mathrm{~W} \\
150 \mathrm{~W}\end{array}$ & $\begin{array}{l}57 \% \\
74 \% \\
78 \%\end{array}$ & $\begin{array}{l}20 \mathrm{~ms} \\
20 \mathrm{~ms} \\
10 \mathrm{~ms}\end{array}$ \\
\hline Moon et al. (2007) & Descarga & - & $78 \%$ & $11 \mathrm{~ms}$ \\
\hline 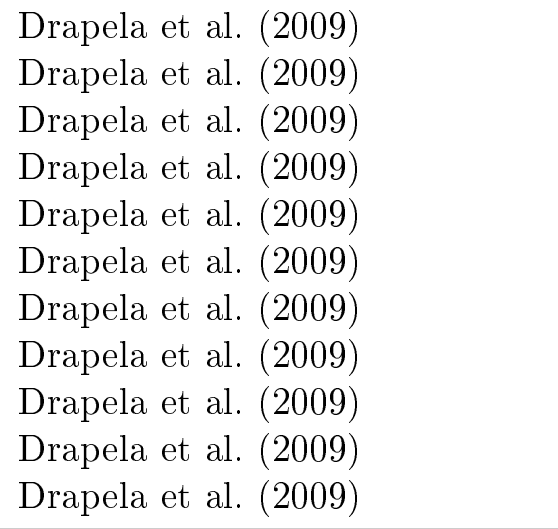 & $\begin{array}{l}\text { eLFL } \\
\text { LFL } \\
\text { eCFL } \\
\text { CFL } \\
\text { VSAP } \\
\text { eLFL } \\
\text { HM } \\
\text { VMAP } \\
\text { LFL } \\
\text { HM } \\
\text { VSAP }\end{array}$ & $\begin{array}{l}58 \mathrm{~W} \\
18 \mathrm{~W} \\
23 \mathrm{~W} \\
13 \mathrm{~W} \\
70 \mathrm{~W} \\
36 \mathrm{~W} \\
400 \mathrm{~W} \\
80 \mathrm{~W} \\
58 \mathrm{~W} \\
150 \mathrm{~W} \\
50 \mathrm{~W}\end{array}$ & $\begin{array}{l}25 \% \\
45 \% \\
55 \% \\
60 \% \\
61 \% \\
65 \% \\
66 \% \\
78 \% \\
78 \% \\
78 \% \\
85 \%\end{array}$ & $\begin{array}{l}60 \mathrm{~ms} \\
20 \mathrm{~ms} \\
28 \mathrm{~ms} \\
8 \mathrm{~ms} \\
6 \mathrm{~ms} \\
10 \mathrm{~ms} \\
10 \mathrm{~ms} \\
7 \mathrm{~ms} \\
8 \mathrm{~ms} \\
5 \mathrm{~ms} \\
7 \mathrm{~ms}\end{array}$ \\
\hline \begin{tabular}{|l|l|} 
Shareef et al. & $(2009 \mathrm{~b})$ \\
Shareef et al. & $(\overline{2009 b})$ \\
\end{tabular} & $\begin{array}{l}\text { eLFL } \\
\text { LFL }\end{array}$ & $\begin{array}{l}18 \mathrm{~W} \\
18 \mathrm{~W} \\
\end{array}$ & - & $\begin{array}{l}15 \mathrm{~ms} \\
7.2 \mathrm{~ms} \\
\end{array}$ \\
\hline 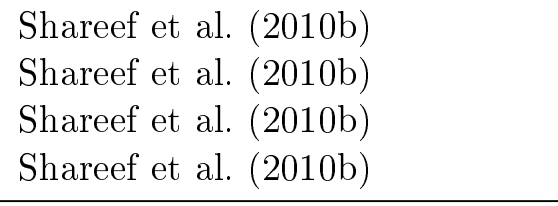 & $\begin{array}{l}\text { eCFL } \\
\text { eCFL } \\
\text { eCFL } \\
\text { eCFL }\end{array}$ & $\begin{array}{l}8 \mathrm{~W} \\
8 \mathrm{~W} \\
14 \mathrm{~W} \\
23 \mathrm{~W}\end{array}$ & $\begin{array}{l}23 \% \\
35 \% \\
27 \% \\
30 \%\end{array}$ & $\begin{array}{l}40 \mathrm{~ms} \\
40 \mathrm{~ms} \\
40 \mathrm{~ms} \\
40 \mathrm{~ms}\end{array}$ \\
\hline \begin{tabular}{|l|l|} 
Hardi y Daut & $(2010)$ \\
Hardi y Daut & $\left(\begin{array}{l}2010 \\
\text { Hardi y Daut }\end{array}\right)$ \\
$\overline{2010}$
\end{tabular} & $\begin{array}{c}\text { CFL } \\
\text { eCFL } \\
\text { VMAP }\end{array}$ & $\begin{array}{c}18 \mathrm{~W} \\
36 \mathrm{~W} \\
250 \mathrm{~W}\end{array}$ & $\begin{array}{l}20 \% \\
30 \% \\
40 \%\end{array}$ & $\begin{array}{l}100 \mathrm{~ms} \\
100 \mathrm{~ms} \\
100 \mathrm{~ms}\end{array}$ \\
\hline
\end{tabular}

Tabla 2.6: Resumen de los principales estudios realizados sobre huecos de tensión en diversas cargas de iluminación. 


\subsection{Descripción de los ensayos y del material em- pleado}

Una vez analizados los trabajos expuestos por diferentes grupos de investigación en el estado del arte presentado en el apartado 2.2, en el presente apartado se describe tanto la variada aparamenta eléctrica ensayada en el apartado 2.4 como el equipo generador de huecos de tensión empleado.

Igualmente, se describe el procedimiento de ensayo diseñado para todo el equipamiento eléctrico analizado.

\subsubsection{Generador de huecos de tensión}

Todos los ensayos realizados en la Tesis Doctoral en relación con la temática abordada en el presente capítulo han sido llevados a cabo con una fuente de alimentación alterna trifásica programable, el modelo 360AMXTUPC 32 de la marca Pacific Power Source. El intercambio de información con la fuente inicialmente sólo era posible desde el panel de control de la misma. Debido a la gran variedad de ensayos llevados a cabo en la Tesis Doctoral, se procedió a la actualización de la misma mediante la sustitución de dos placas de circuito impreso. Gracias a esta mejora de hardware es posible el control de la fuente de alimentación desde un $\mathrm{PC}$ mediante el software proporcionado por el fabricante, conocido como UPC Studio.

Mediante esta fuente de alimentación se puede obtener una tensión de salida monofásica, bifásica o trifásica, a una tensión y frecuencia modificable en función de las necesidades del usuario. También, la forma de la onda de salida puede ser seleccionada en función del estudio que se quiera realizar. Sin embargo, los límites admisibles dependen de la conexión que se realice:

- Modo "Acoplamiento Directo": esta es la configuración por defecto de la fuente. El rango de la tensión de línea de salida varía entre $0 V_{a c}$ y $234 V_{a c}$; mientras que el rango de frecuencias se sitúa entre $20 \mathrm{~Hz} \mathrm{y}$ $5 \mathrm{kHz}$.

- Modo "Acoplamiento Mediante Transformador": esta configuración es una opción añadida a la configuración de serie. De esta manera, se consigue que la tensión de línea de salida dependa de la relación de transformación impuesta por el transformador. Concretamente, se pueden seleccionar hasta tres relaciones de transformación diferentes, pudiéndose conseguir una tensión máxima de línea de salida de $585 V_{a c}$. Sin embargo, el rango de frecuencias disponibles resulta mermado, situándose entre $45 \mathrm{~Hz}$ y $5 \mathrm{kHz}$.

Independientemente del modo de conexión seleccionado, la fuente posee una potencia nominal de $6 \mathrm{kVA}$. La configuración disponible contaba con 


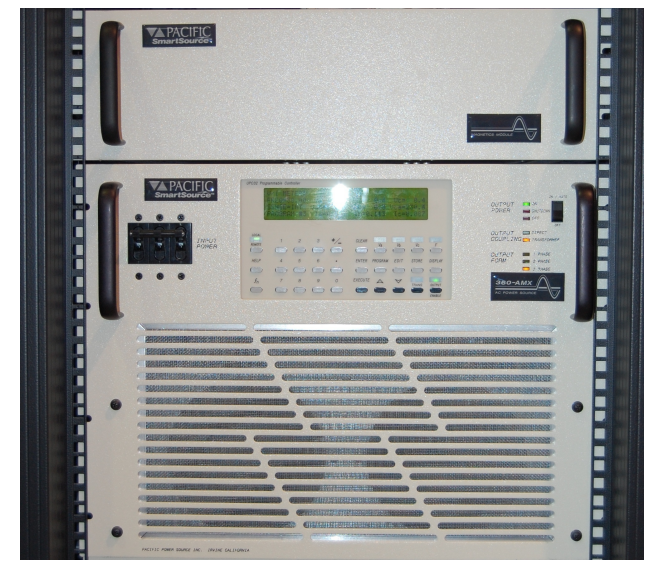

Figura 2.12: Vista frontal de la fuente de potencia.

cuatro fuentes de alimentación de este tipo conectadas en paralelo, de manera que se puede llegar a entregar una potencia total de $24 \mathrm{kVA}$. La figura 2.12 muestra una imagen de la fuente de alimentación que se comporta como "maestra" — dado que las tres restantes trabajan en modo "esclavo"- junto con el transformador asociado a la misma alojado sobre ella.

Además de la fuente de alimentación, la plataforma de ensayos contaba también con un osciloscopio, concretamente un Yokogawa DL9710L, junto a una sonda de tensión diferencial para ajustar la tensión de entrada al mismo. De esta manera, las formas de onda de la tensión definidas a través del software UPC Studio son monitorizadas por el osciloscopio.

Por último, para que los ensayos no comprometan la seguridad de las personas ni del equipamiento ensayado, la conexión entre la fuente de alimentación y los equipos se realiza a través de un cuadro eléctrico diseñado específicamente para esta aplicación, contando con los siguientes elementos de maniobra y protección:

- Un interruptor automático de 4P, $25 \mathrm{~A}$.

- Un interruptor diferencial de 4P, 30mA, $25 \mathrm{~A}$.

- Tres interruptores automáticos de 4P y 16 A.

- Un pulsador de marcha y otro de parada.

\subsubsection{Características del equipamiento ensayado}

Tal y como se comentó en el apartado 2.2 , los ensayos han sido realizados sobre una diversidad de equipamiento eléctrico presente, tanto en centrales de generación eólica como en los sistemas de energía eléctrica, siendo: 
- Contactores.

- Relé de falta a tierra.

- Variador de frecuencia.

- Ordenador personal.

- Diferentes cargas de iluminación.

Por lo tanto, para que los resultados obtenidos en la presente Tesis Doctoral puedan ser comparados con los que se han encontrado en la extensa revisión bibliográfica realizada en el apartado 2.2 , a continuación se describen las características técnicas de cada equipo eléctrico ensayado.

\subsubsection{Características de los contactores}

Con el objeto de comprobar que la respuesta de este tipo de aparamenta eléctrica puede verse afectada por el fabricante de la misma, se han ensayado tres contactores de corriente alterna de diversos fabricantes que presentan unas especificaciones técnicas muy similares:

- Contactor Telemecanique LC1-D09.

- Contactor Schneider Electric LC1-D09.

- Contactor Mitsubishi S-N10.

Aunque en el circuito de potencia de los parques eólicos se empleen contactores de mayor capacidad que los que se han ensayado en la presente Tesis Doctoral, los contactores listados anteriormente son comúnmente empleados para la maniobra de gran parte de los equipos auxiliares presentes en centrales de generación de energía eólica.

En la tabla 2.7 se resumen las especificaciones técnicas más importantes de los tres contactores ensayados en función de las definiciones realizadas en el apartado 2.2.1.

Aunque se comentará más adelante, los ensayos con contactores han sido realizados bajo diferentes hipótesis de carga, empleando tanto cargas resistivas, como inductivas y capacitivas. Por lo tanto, resulta necesario nombrar a estas tres cargas.

En el caso de las resistencias, se han utilizado estufas eléctricas de laboratorio, modelo Wunder-1, de $400 \mathrm{~W}$ y $230 \mathrm{~V}$ de potencia y tensión nominal, respectivamente. De esta manera, mediante la conexión en estrella de estas cargas, la ecuación 2.3 muestra la intensidad que atraviesa el contactor:

$$
I=\frac{P_{t o t a l}}{\sqrt{3} \cdot V_{L L}}=\frac{1200}{\sqrt{3} \cdot 400}=1,73 \mathrm{~A}
$$




\begin{tabular}{|c|c|c|c|}
\hline Propiedad & Telemecanique & Schneider & Mitsubishi \\
\hline $\begin{array}{l}\text { Tensión nominal de } \\
\text { aislamiento }\end{array}$ & $1000 \mathrm{~V}$ & $690 \mathrm{~V}$ & $690 \mathrm{~V}$ \\
\hline \multicolumn{4}{|c|}{ Intensidad nominal de empleo } \\
\hline Categoría AC3 & $9 \mathrm{~A}$ & $9 \mathrm{~A}$ & $9 \mathrm{~A}$ \\
\hline Categoría AC1 & $25 \mathrm{~A}$ & $25 \mathrm{~A}$ & $20 \mathrm{~A}$ \\
\hline Número de polos & 3 & 3 & 3 \\
\hline \multicolumn{4}{|c|}{ Potencia nominal en AC3 } \\
\hline $220 / 240 \mathrm{~V}$ & $2.2 \mathrm{~kW}$ & $2.2 \mathrm{~kW}$ & $2.5 \mathrm{~kW}$ \\
\hline $380 / 400 \mathrm{~V}$ & $4 \mathrm{~kW}$ & $4 \mathrm{~kW}$ & $4 \mathrm{~kW}$ \\
\hline $\begin{array}{l}\text { Tensión nominal del } \\
\text { circuito de control }\end{array}$ & $230 V_{a c}$ & $230 V_{a c}$ & $230 V_{a c}$ \\
\hline
\end{tabular}

Tabla 2.7: Características de los contactores ensayados.

De manera similar, con el objeto de variar la intensidad de carga, conectando dos resistencias en serie se obtiene una intensidad de $0.87 \mathrm{~A}$, ecuación (2.4); mientras que conectando dos resistencias en paralelo la intensidad que atraviesa el contactor asciende a $3.46 \mathrm{~A}$, ecuación (2.5).

$$
\begin{gathered}
I_{\text {serie }}=\frac{P_{\text {total }}}{\sqrt{3} \cdot V_{L L}}=\frac{600}{\sqrt{3} \cdot 400}=0,87 \mathrm{~A} \\
I_{\text {paralelo }}=\frac{P_{\text {total }}}{\sqrt{3} \cdot V_{L L}}=\frac{2400}{\sqrt{3} \cdot 400}=3,46 \mathrm{~A}
\end{gathered}
$$

En relación con las cargas inductivas, se han ensayado reactancias del fabricante ELT, modelo VMI 25/22-3, empleadas típicamente para la alimentación de lámparas de vapor de mercurio. Finalmente, como carga capacitiva se posee un banco de condensadores del fabricante $A B B$, modelo CLMD 33, empleado comúnmente en instalaciones industriales para corrección del factor de potencia.

Finalmente, como se podrá observar en el apartado 2.4, la respuesta de contactores ante huecos de tensión se encuentra relacionada con su temperatura de operación. Por este motivo, se ha empleado un termopar para medir la temperatura de la carcasa del contactor — dado que esta temperatura es representativa de la temperatura de la bobina-. Concretamente se trata de un modelo $80 \mathrm{PK}$-1, de la marca Fluke, que permite medir temperatura desde $-40{ }^{\circ} \mathrm{C}$ hasta $260{ }^{\circ} \mathrm{C}$. 


\subsubsection{Características del relé de falta a tierra}

El relé de falta a tierra ensayado es de la marca Merlin Gerin, modelo Vigirex RH197P. Se ha empleado en combinación con un transformador de intensidad modelo MA120. Las especificaciones técnicas se muestran en la tabla 2.8

\begin{tabular}{l|c}
\hline Propiedad & \\
\hline Tensión nominal de alimentación & $220-240 V_{a c}$ \\
\hline Intensidad nominal del CT & $250 \mathrm{~A}$ \\
\hline
\end{tabular}

Tabla 2.8: Características del relé de falta a tierra ensayado.

Como se puede observar en la tabla 2.8, el relé de falta a tierra ensayado, aunque es alimentado en baja tensión, la intensidad que puede llegar a monitorizar es elevada, pudiéndose emplear, por lo tanto, para prevenir las faltas a tierra en el circuito de potencia de un parque eólico.

\subsubsection{Características del variador de frecuencia}

El variador de frecuencia ensayado pertenece al fabricante Reliance Electric, modelo SP 200, caracterizado por su elevado uso para el control de pequeños motores de corriente alterna en baja tensión. Concretamente, se emplea sobre motores trifásicos, mientras que su alimentación es monofásica. Sus especificaciones técnicas se muestran en la tabla 2.9 Este tipo de equipamiento es comúnmente empleado en parques eólicos para el control de algunos componentes auxiliares de los mismos. Por ejemplo, el sistema de control del ángulo de giro de las palas — conocido como control de pitch, descrito en el apartado 4.3.3 , habitualmente emplea variadores de frecuencia trifásicos, $\mathrm{Hau}$ (2005).

Con este variador de frecuencia se ha alimentado una máquina asíncrona de la empresa Lucas Nülle, de $400 \mathrm{~V}$ de tensión nominal, y un tacómetro

\begin{tabular}{l|c}
\hline Propiedad & \\
\hline Tensión nominal de alimentación & $200-240 V_{a c}$ \\
Intensidad nominal de alimentación & $14.3 \mathrm{~A}$ \\
\hline Tensión nominal de salida & $0-230 V_{a c}$ \\
Intensidad nominal de salida & $7 \mathrm{~A}$ \\
\hline
\end{tabular}

Tabla 2.9: Características del variador de frecuencia ensayado. 
para medir la velocidad de giro del motor.

\subsubsection{Características del ordenador personal}

Las características técnicas del PC ensayado se muestran en la tabla 2.10. Aunque la información mostrada en dicha tabla podría suponer que el $\mathrm{PC}$ ensayado es antiguo, ha servido para contrastar los resultados obtenidos por otros autores, presentados en el apartado 2.2.4 Además, dado que España cuenta con un elevado número de parques eólicos que poseen cierta antigüedad, se puede considerar que los $\mathrm{PC}$; que se continúan empleando para la monitorización de dichos parques poseen similares propiedades a las descritas en la tabla 2.10 ,

Por lo tanto, se puede considerar que el $\mathrm{PC}$ ensayado representa el tipo de $\mathrm{PC}$ que se sigue empleando en una proporción relativamente elevada de parques eólicos.

\begin{tabular}{l|c}
\hline Propiedad & \\
\hline Microprocesador & Intel $80486,100 \mathrm{MHz}$ \\
\hline Memoria RAM & $12 \mathrm{MB}$ \\
\hline Sistema operativo & Windows 98 \\
\hline Disco duro & $2 \mathrm{~GB}$ \\
\hline Tensión nominal de alimentación & $230 V_{a c}$ \\
\hline
\end{tabular}

Tabla 2.10: Características del ordenador personal ensayado.

\subsubsection{Características de las cargas de iluminación}

Por último, también se han ensayado frente a huecos de tensión e interrupciones diversas cargas de iluminación, con el objetivo de abarcar diferentes tecnologías.

En el caso de la iluminación fluorescente, se han realizado ensayos sobre un tubo fluorescente de $58 \mathrm{~W}$ de potencia nominal de la marca General Electric. Este tubo ha sido conectado a través de balasto electromagnético en una serie de ensayos, así como de balasto electrónico en otros ensayos.

Los ensayos realizados sobre la tecnología de inducción incluyen una lámpara de $165 \mathrm{~W}$ de potencia nominal del fabricante Philips alimentada por un balasto electrónico ofrecido por el mismo fabricante.

Por último, la lámpara de halogenuros metálicos que se ha empleado en los ensayos posee una potencia nominal de $150 \mathrm{~W}$ de la marca Osram, conectada a través de un balasto electromagnético. 


\subsubsection{Descripción del procedimiento de ensayo}

Con el objetivo de obtener unos resultados contrastables a los que han presentado otros autores, se ha seguido un riguroso procedimiento de ensayo. Aunque todo el equipamiento ensayado se ha realizado bajo un procedimiento prácticamente idéntico, concretamente en el caso de los contactores se ha procedido de la siguiente manera:

1. En primera instancia, se cierran los contactos principales del contactor mediante el suministro de una tensión senoidal de $230 V_{a c}$ a su circuito de control.

2. El paso anterior se mantiene en el tiempo hasta que se consigue una temperatura de servicio de $45{ }^{\circ} \mathrm{C}$; dado que una vez alcanzado este valor, se mantiene estable. De esta manera se analiza la respuesta del contactor en las condiciones óptimas de funcionamiento. En el apartado 2.4.1 se ha comprobado que esta temperatura no resulta modificada por los ensayos realizados.

3. Cuando se alcanza la temperatura de servicio, se puede comenzar a realizar ensayos, es decir, aplicar las formas de onda previamente introducidas mediante el software UPC Studio en la fuente de alimentación. El primer ensayo se comienza con un ángulo de inicio de la onda de tensión igual a $0^{\circ}$.

4. A continuación se realiza un test a una profundidad y duración de hueco establecidas. Específicamente, los ensayos comienzan con el valor $0 \%$ de tensión residual (100\% profundidad de hueco).

5. Cada test se repite 10 veces con el interés de observar la existencia de bandas de dispersión en la respuesta del equipo.

6. Sin modificar la tensión residual del hueco, la duración del mismo se incrementa progresivamente hasta que 9 de las 10 veces ensayadas se produce la desconexión del equipo.

7. Ahora se aumenta la tensión residual. Concretamente, la tensión residual es incrementada en escalones del $5 \%$ de la tensión nominal.

8. De esta manera, después de un elevado número de ensayos se cuenta con la curva de tolerancia del contactor a un ángulo de inicio de la onda de tensión determinado; por lo que es necesario aumentar este valor y volver al paso 3. Concretamente, el ángulo de inicio de la onda de tensión se ha variado en escalones de $15^{\circ}$.

Con el objeto de aclarar el procedimiento de realización de ensayos descrito en las líneas anteriores se ha realizado un diagrama de flujo del mismo, figura 2.13 . 


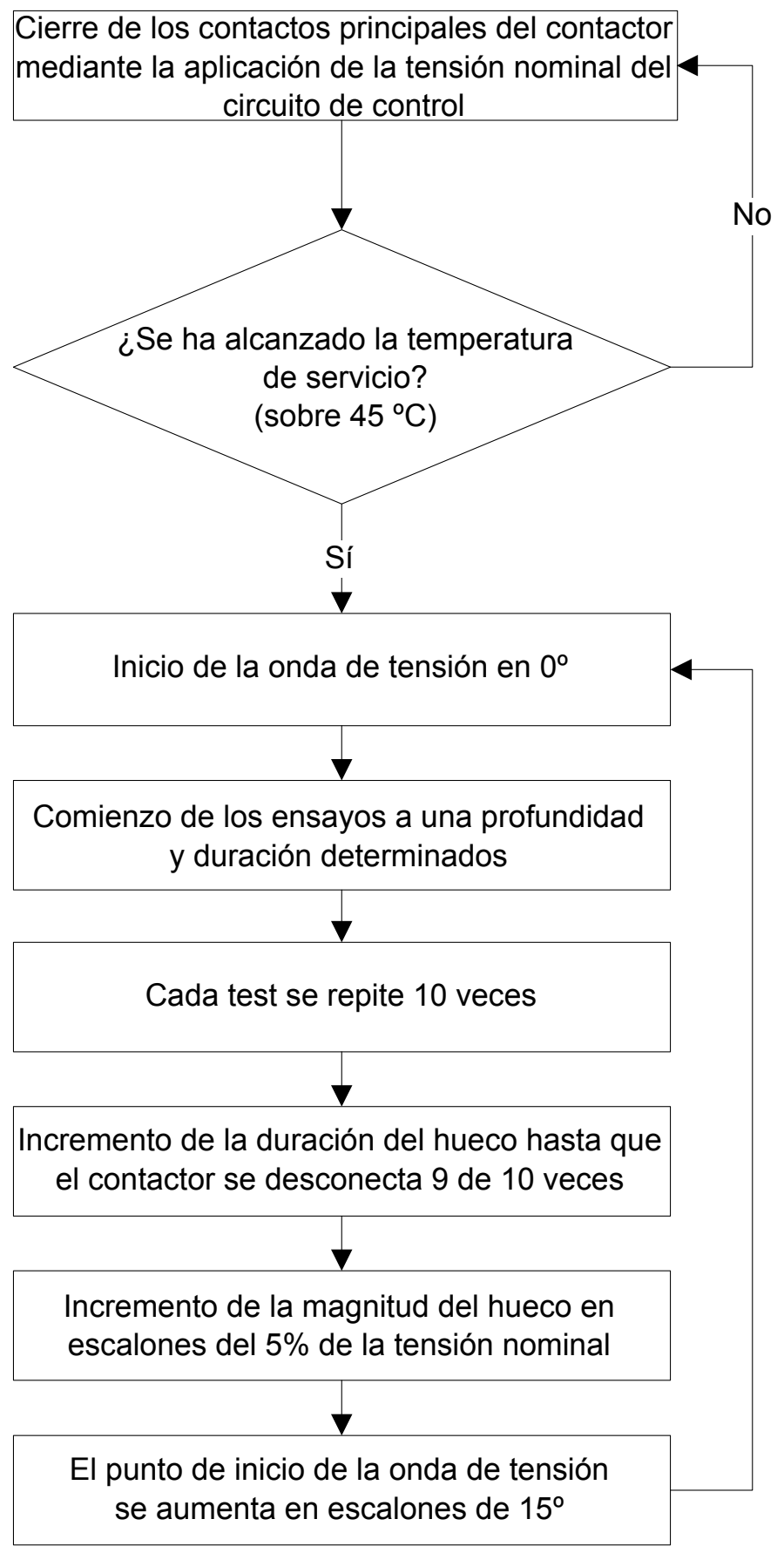

Figura 2.13: Diagrama de flujo del procedimiento de ensayo diseñado. 


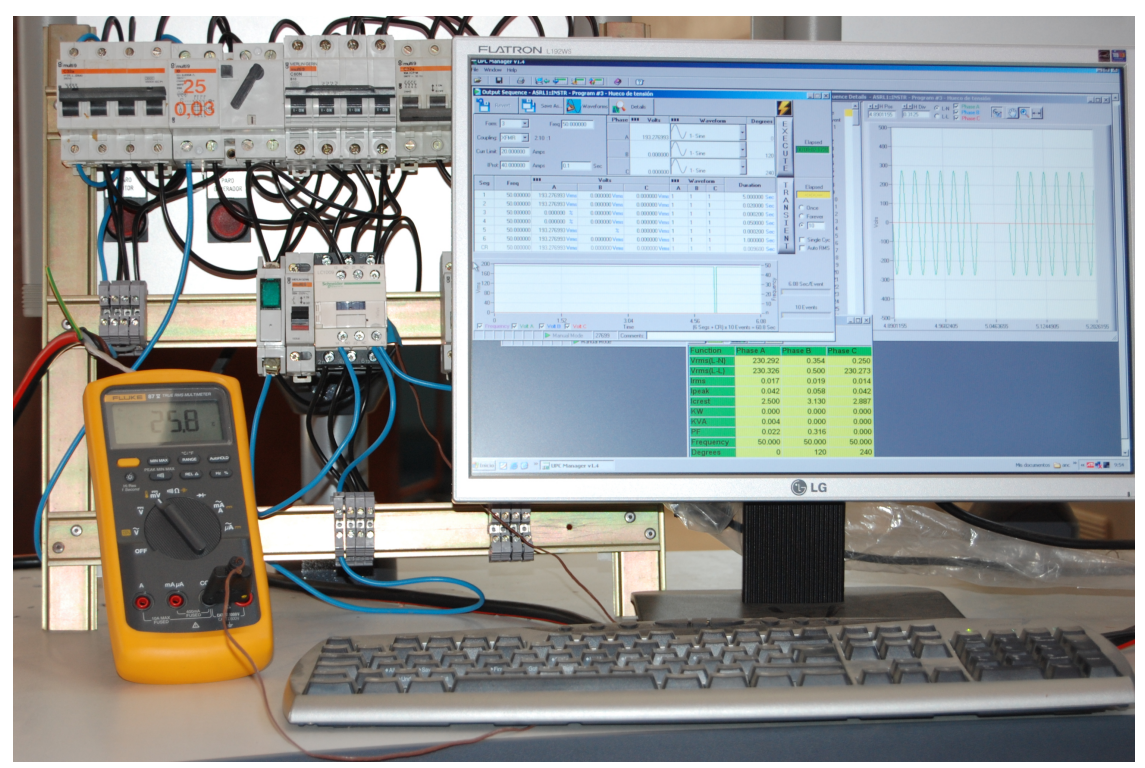

Figura 2.14: Ejemplo de ensayo experimental.

Para concluir el presente apartado, que se ha dedicado a la especificación de equipos así como al diseño del procedimiento de ensayo experimental, la figura 2.14 resume las ideas que se han comentando en el mismo. Concretamente, dicha figura muestra un ejemplo de preparación de los ensayos llevados a cabo sobre los contactores.

En la parte derecha de la figura 2.14 se muestra el monitor mediante el cual se interactúa con el software UPC Studio que controla la fuente de alimentación. Particularmente, se puede observar la forma de onda de una interrupción del suministro de $50 \mathrm{~ms}$ de duración que comienza en los $0^{\circ}$ de la onda de tensión. Puede verse que esta onda de tensión se encuentra programada para ser ejecutada diez veces sobre el equipo a ensayar. En la parte superior izquierda de la figura 2.14 se tiene el cuadro eléctrico diseñado teniendo en cuenta la seguridad de los equipos y de las personas, descrito en el apartado 2.3.1. Por último, en la zona inferior izquierda se observa el multímetro empleado para monitorizar la temperatura de operación del contactor. Se observa cómo, en el momento en que se hizo la fotografía, el contactor se encontraba a una temperatura de servicio similar a la temperatura ambiente, dado que aún no se había iniciado el procedimiento de ensayo descrito en el presente apartado. 


\subsection{Resultados}

Una vez revisados los aportes científicos encontrados en la revisión bibliográfica presentada en el apartado 2.2, y definido el equipamiento y procedimiento de ensayo en el apartado 2.3. el presente apartado contiene todos los resultados de los experimentos realizados en la Tesis Doctoral dentro de la temática introducida en el presente capítulo.

Primeramente, se van a señalar algunos aspectos generales. Tal y como se indicó en el apartado 2.1.2, las diferentes Normas que se conocen relativas a la realización de ensayos para la obtención de curvas de tolerancia indican que los ensayos deben realizarse solamente bajo determinadas tensiones residuales $y$, preferiblemente, en el instante en que la tensión se sitúa en $0^{\circ}$. Como se observa más adelante en el extenso desarrollo experimental llevado a cabo en la presente Tesis Doctoral, las consideraciones anteriores no son coherentes cuando se aplican sobre determinado equipamiento eléctrico.

Por lo tanto, a continuación se exponen los resultados obtenidos en los experimentos realizados sobre cada tipo de equipamiento eléctrico.

\subsubsection{Ensayos realizados sobre contactores}

En el caso de este tipo de aparamenta eléctrica, se ha profundizado especialmente en analizar su comportamiento frente a diversas perturbaciones que ocurren en las redes eléctricas, dado que su uso en centrales de generación de energía eólica así como en cualquier sistema de energía eléctrica se encuentra ampliamente extendido. Además, algunos autores, como Turner y Collins (1996); Collins y Bridgwood (1997); Collins y Zapardiel (1997), señalan que los contactores exhiben un comportamiento relativamente aleatorio ante huecos de tensión; mientras que otros, como Djokic et al. (2004); Akolkar y Kushare (2010), ponen de manifiesto la compleja respuesta de este tipo de aparamenta eléctrica.

Por este motivo, se han realizado ensayos tanto en vacío como en carga de los tres contactores descritos en el apartado 2.3.2.1. Es importante observar que la variedad de cargas empleadas — resistivas, inductivas y capacitivaspermite variar en un amplio rango el módulo y el desfase de la intensidad que atraviesa el circuito de potencia del contactor. Además, se ha variado el ángulo inicio de la onda de tensión. Igualmente, otro aspecto que no ha sido considerado por otros autores es el efecto de la temperatura de operación de esta aparamenta sobre su respuesta frente a huecos de tensión; por lo que también es objeto de análisis.

Adicionalmente, se desarrolla también un estudio de probabilidad de disparo que permite analizar la respuesta de cada contactor con mayor precisión en cada instante. De esta manera, cada rectángulo de cada curva de tolerancia que se presentará más adelante representa diez ensayos realizados sobre 
el contactor de una magnitud y duración del hueco determinadas. Cada rectángulo está coloreado para indicar la probabilidad de fallo, simbolizando el color rojo oscuro que se ha producido la desconexión del contactor diez veces; mientras que el color azul oscuro significa que no se produce la desconexión del equipo en ninguno de los diez ensayos a la tensión residual y duración establecidas. No obstante, se ha incluido una leyenda junto a cada gráfico que indica la probabilidad de fallo en tanto por ciento.

De esta manera, todas las curvas de tolerancia obtenidas en contactores presentan tres zonas claramente diferenciadas en función de su color:

- Zona de disparo seguro: caracterizada por un color rojo oscuro, donde la probabilidad de desconexión del equipo es del $100 \%$. Es decir, en esta zona, independientemente del hueco de tensión que se produzca, el contactor abrirá sus contactos. Por lo tanto, cuanto menor área ocupe esta zona, menor sensibilidad posee el contactor ante huecos de tensión e interrupciones.

- Zona de no disparo seguro: representada por un color azul oscuro, donde sea cual sea la profundidad y duración del la perturbación, no se modifica el estado del contactor.

- Zona de respuesta inestable o zona de dispersión: en esta zona no se puede predecir la respuesta del contactor ante las perturbaciones ensayadas. En la extensa revisión bibliográfica realizada no se ha encontrado ningún trabajo que mencione este tipo de comportamiento.

Bajo las anteriores consideraciones, se está en condiciones de afirmar que el profundo análisis experimental efectuado sobre este tipo de aparamenta eléctrica ha generado más de 40.000 ensayos sobre contactores.

\subsubsection{Ensayos en vacío}

A continuación se presentan las curvas de tolerancia obtenidas sobre los diferentes contactores en función del ángulo de inicio de la onda de tensión cuando no se encontraba ninguna carga conectada a su salida.

De esta manera, las figuras 2.15, 2.16 y 2.17 muestran las diferentes curvas de tolerancia obtenidas para los contactores Schneider, Telemecanique y Mitsubishi, respectivamente. Tal y como se observa en estas figuras, se ha seguido el procedimiento de ensayo descrito en el apartado 2.3.3 y esquematizado en la figura 2.13, variando el ángulo de inicio de la onda de tensión desde $0^{\circ}$ (subfiguras (a) hasta $90^{\circ}$ (subfiguras (g) , en escalones de $15^{\circ}$ (subfiguras (b), (c), (d), (e), (f) . Como se indicó anteriormente, los colores que aparecen en cada figura representan probabilidad de fallo - o desconexión involuntaria- del equipo. Por ejemplo, en la gráfica $2.15 \mathrm{~g}$ para una profundidad del hueco de tensión del $95 \%$ y una duración de 10 ms — técnicamente 
es una interrupción del suministro-, se observa que la probabilidad de que se produzca el disparo del contactor Schneider es del $60 \%$.

Una vez obtenidas las gráficas mostradas en las figuras 2.15, 2.16 y 2.17, se está en condiciones de señalar las primeras observaciones. La morfología de las curvas ensayadas es muy similar a la que se comentó en la revisión bibliográfica del apartado 2.2.1. Las curvas de tolerancia del contactor varían significativamente en función del instante de inicio de la onda donde se aplique el hueco de tensión. De manera general, se puede afirmar que para ángulos de inicio de la onda de tensión comprendidos entre $0^{\circ}$ y $60^{\circ}$, este tipo de aparamenta eléctrica se caracteriza de modo que a medida que aumenta la profundidad del hueco también aumenta el tiempo que el equipo puede soportar dicha perturbación; mientras que para ángulos comprendidos entre $75^{\circ}$ y $90^{\circ}$ los tiempos de disparo son mínimos independientemente de la profundidad del hueco. De hecho, se puede comprobar que las gráficas (f) $\mathrm{y}(\mathrm{g})$ de las figuras 2.15, 2.16 y 2.17, se corresponden con curvas de tolerancia prácticamente rectangulares.

Por lo tanto, se puede comprobar que los contactores ensayados son más sensibles, frente a huecos de tensión profundos, cuando el inicio de la onda de tensión se aproxima a $90^{\circ}$ que cuando se aproxima a $0^{\circ}$. Un ejemplo relevante de esta situación se puede encontrar en el contactor Telemecanique, figura 2.16. Si se produce una interrupción del suministro sobre este contactor cuando la onda de tensión se encuentra en $0^{\circ}$, soportará $61 \mathrm{~ms}$ sin desconectar; mientras que si la misma interrupción comienza a $90^{\circ}$, sólo se asegura el correcto funcionamiento del equipo si la perturbación no supera $9 \mathrm{~ms}$.

Sin embargo, se observa en las figuras 2.15, 2.16 y 2.17 que ante huecos de tensión de menor profundidad y mayor duración, menores ángulos de inicio de la onda de tensión implican un aumento de la sensibilidad de este tipo de aparamenta. Concretamente, se puede observar que la profundidad que pueden soportar cualquiera de los tres contactores ensayados frente a huecos de tensión de elevada duración resulta aumentada conforme crece el ángulo de inicio de la onda de tensión.

Como se indicó anteriormente, una contribución importante que no se ha encontrado en la bibliografía especializada es la existencia de una zona de dispersión, donde la respuesta del contactor es considerablemente variable. Un ejemplo concreto de este fenómeno se observa en la curva de tolerancia 2.17b En particular, en este ensayo se observa claramente que huecos de tensión con una profundidad del $50 \%$ y duraciones comprendidas entre $15 \mathrm{~ms}$ y $100 \mathrm{~ms}$ causan que no se pueda predecir la respuesta del contactor ante huecos de tensión. Además, sucede que en el rango de duraciones definido anteriormente, la probabilidad de fallo del equipo no aumenta de manera progresiva, sino que es variable. Por lo menos, se puede observar que esta imprecisión en el comportamiento de los contactores prácticamente 


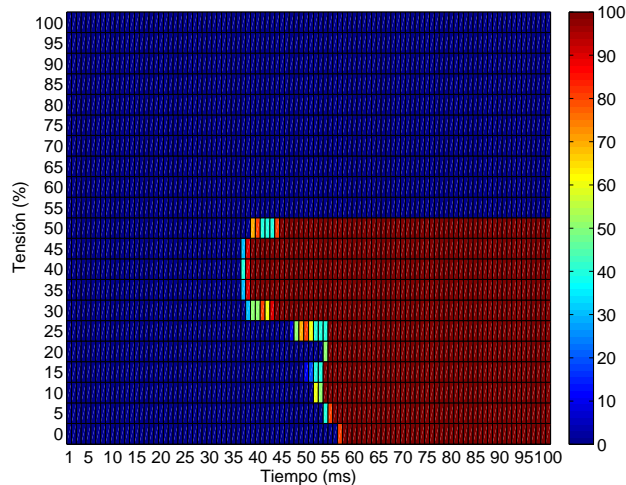

(a) Probabilidad de fallo para $0^{\circ}$.

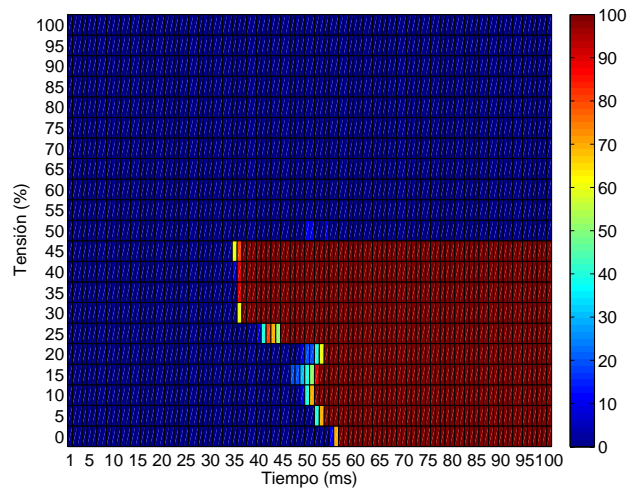

(c) Probabilidad de fallo para $30^{\circ}$.

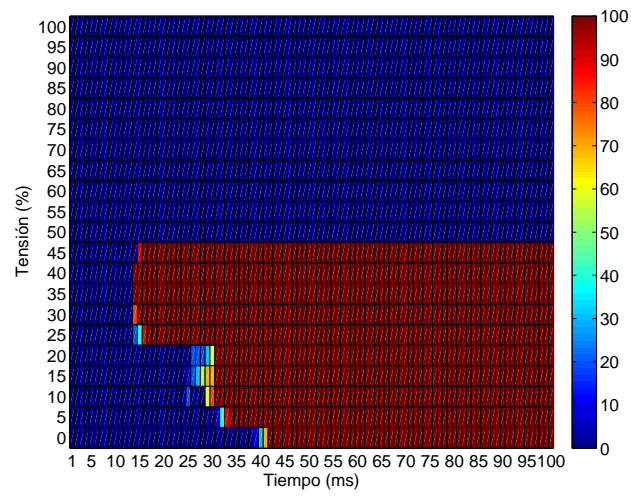

(e) Probabilidad de fallo para $60^{\circ}$.

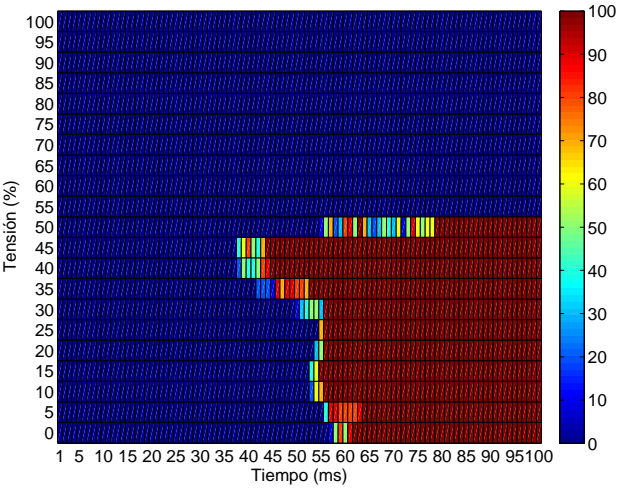

(b) Probabilidad de fallo para $15^{\circ}$.

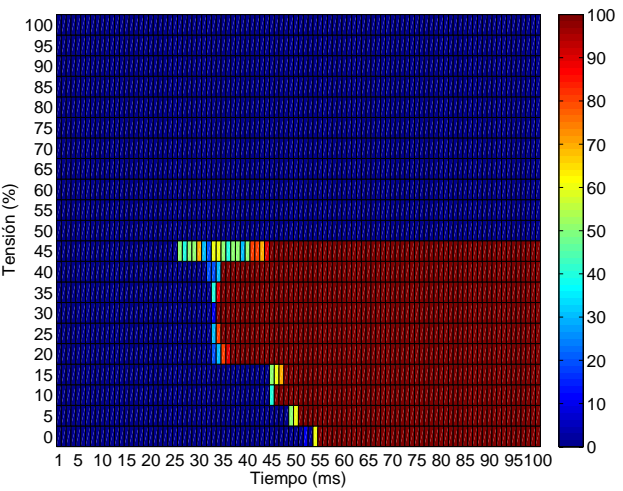

(d) Probabilidad de fallo para $45^{\circ}$.

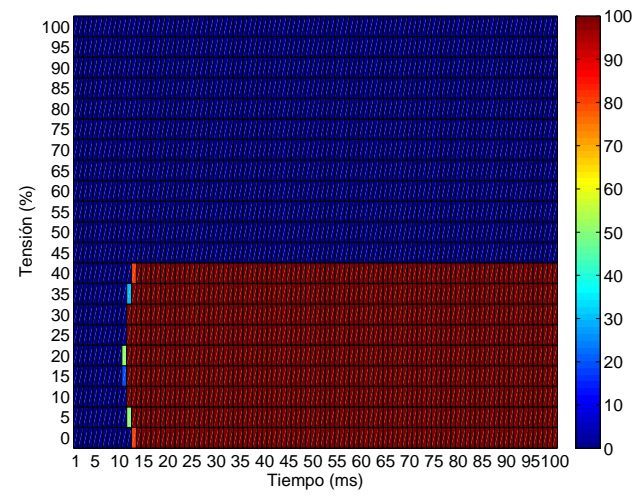

(f) Probabilidad de fallo para $75^{\circ}$.

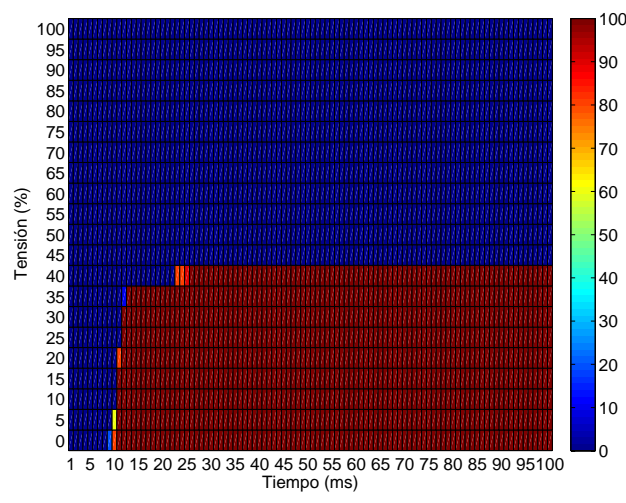

(g) Probabilidad de fallo para $90^{\circ}$.

Figura 2.15: Curvas de tolerancia del contactor Schneider en vacío. 


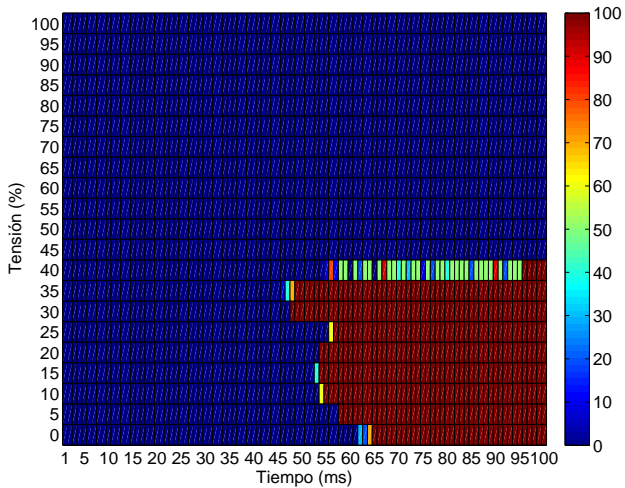

(a) Probabilidad de fallo para $0^{\circ}$.

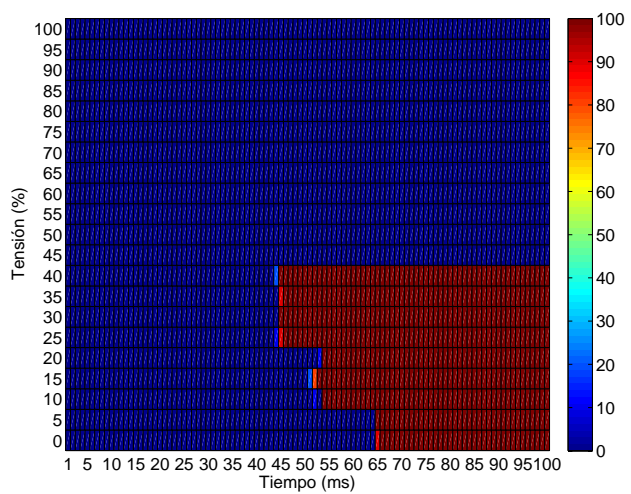

(c) Probabilidad de fallo para $30^{\circ}$.

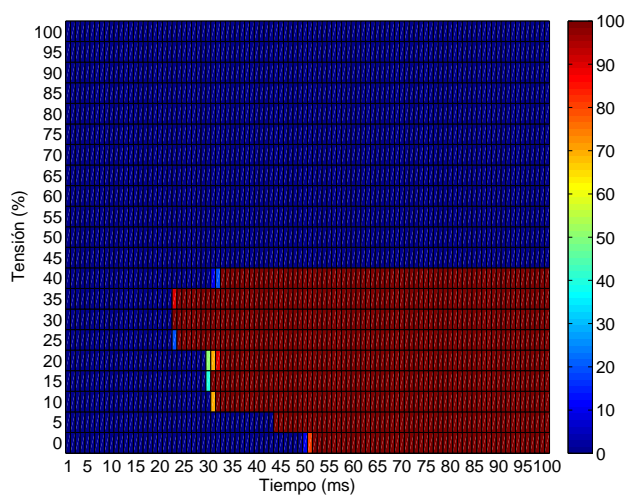

(e) Probabilidad de fallo para $60^{\circ}$.

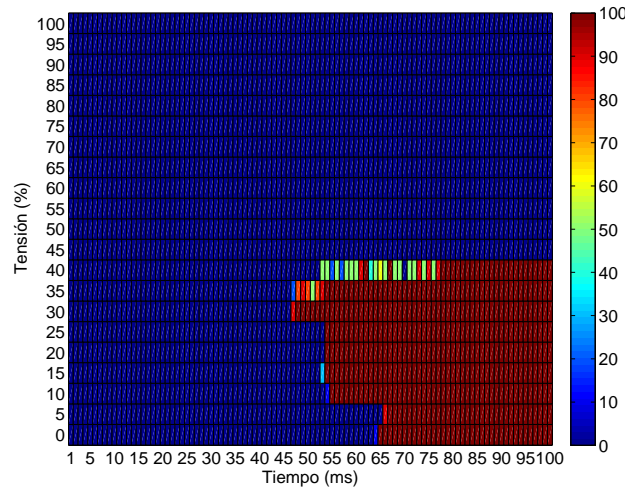

(b) Probabilidad de fallo para $15^{\circ}$.

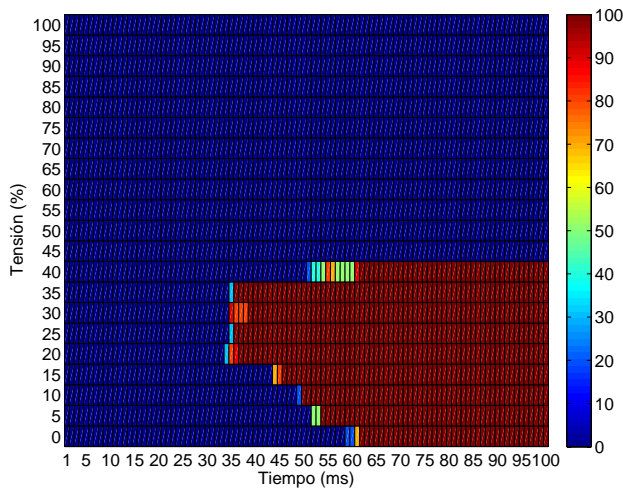

(d) Probabilidad de fallo para $45^{\circ}$.

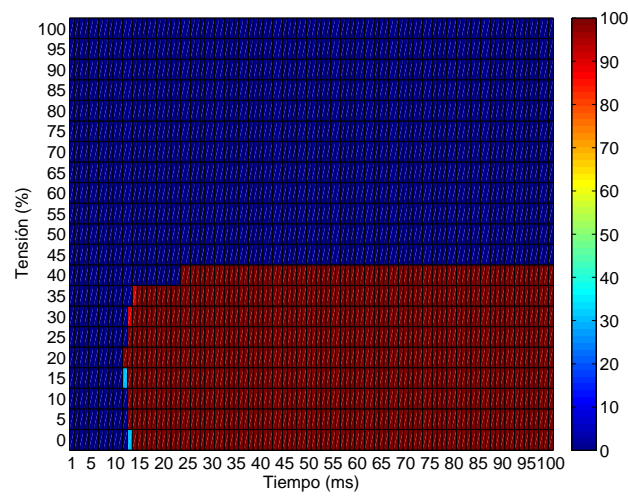

(f) Probabilidad de fallo para $75^{\circ}$.

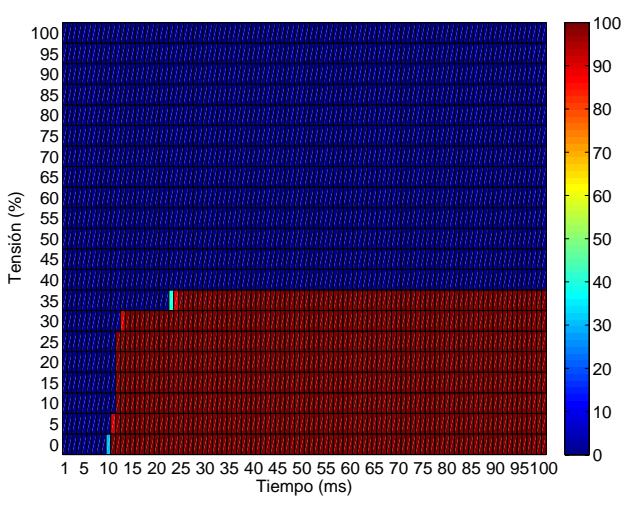

(g) Probabilidad de fallo para $90^{\circ}$.

Figura 2.16: Curvas de tolerancia del contactor Telemecanique en vacío. 


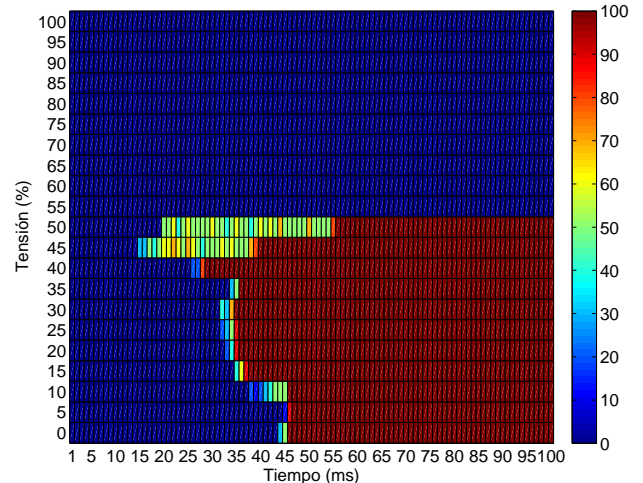

(a) Probabilidad de fallo para $0^{\circ}$.

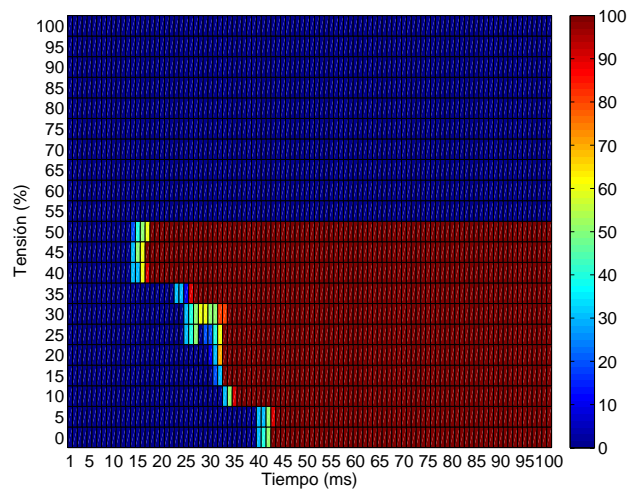

(c) Probabilidad de fallo para $30^{\circ}$.

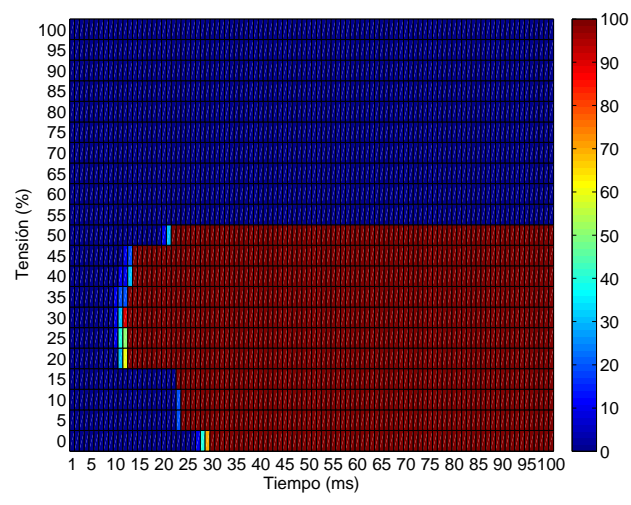

(e) Probabilidad de fallo para $60^{\circ}$.

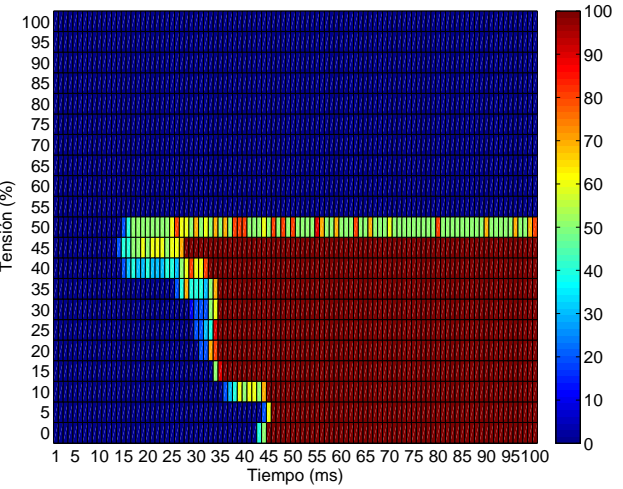

(b) Probabilidad de fallo para $15^{\circ}$.

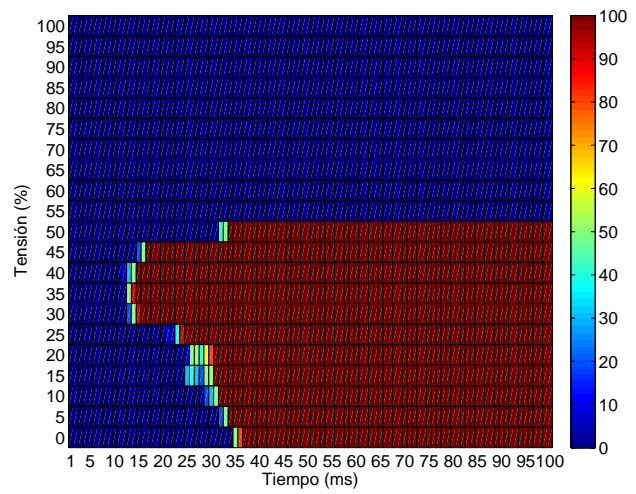

(d) Probabilidad de fallo para $45^{\circ}$.

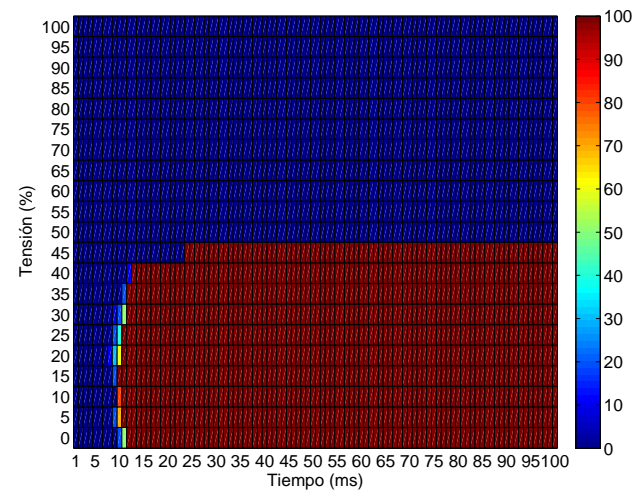

(f) Probabilidad de fallo para $75^{\circ}$.

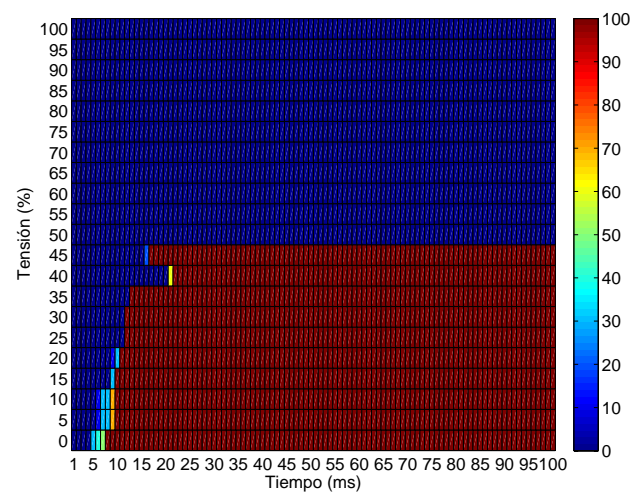

(g) Probabilidad de fallo para $90^{\circ}$.

Figura 2.17: Curvas de tolerancia del contactor Mitsubishi en vacío. 


\begin{tabular}{l|c|c|c|c}
\hline Contactor & $V_{n c_{\min _{90} 0}}$ & $V_{n c_{\min _{0} 0}}$ & $t_{\max _{90^{0}}}$ & $t_{\max _{0^{0}}}$ \\
\hline Schneider & $40 \%$ & $50 \%$ & $11 \mathrm{~ms}$ & $58 \mathrm{~ms}$ \\
\hline Telemecanique & $35 \%$ & $40 \%$ & $11 \mathrm{~ms}$ & $65 \mathrm{~ms}$ \\
\hline Mitsubishi & $45 \%$ & $50 \%$ & $8 \mathrm{~ms}$ & $46 \mathrm{~ms}$ \\
\hline
\end{tabular}

Tabla 2.11: Resumen de los ensayos realizados sobre los tres contactores en vacío.

desaparece cuando el ángulo de inicio de la onda de tensión supera $60^{\circ}$ en los tres contactores ensayados. Continuando con la misma línea, se puede observar en las figuras 2.15, 2.16 y 2.17 que, de manera general, la banda de dispersión sólo aparece en las regiones frontera, es decir, en los instantes en que el contactor pasa de no disparar el $100 \%$ de las veces a disparar el $100 \%$ de los ensayos. Además, en la mayoría de curvas de tolerancia obtenidas, bajo reducidos ángulos de inicio de la onda de tensión, ocurre que la zona de dispersión es relativamente extensa para valores entre el 40-50\% de la tensión nominal.

En función de los ensayos en vacío realizados sobre los tres contactores de corriente alterna se puede afirmar que ninguno de ellos dispara bajo huecos de tensión con una tensión residual superior al $50 \%$, sea cual sea su duración. Este dato se encuentra en línea con la información expuesta en la tabla 2.3 en base a la revisión bibliográfica realizada. Concretamente, los valores específicos obtenidos en los ensayos realizados en la Tesis se han resumido en la tabla 2.11.

Además, también se han realizado ensayos para observar el fenómeno de la simetría de cuarto de ciclo, siendo comprobado que los resultados obtenidos en vacío a $90^{\circ}$ de inicio de la onda de tensión son idénticos que los que se consiguen a $270^{\circ}$.

Hasta ahora sólo se ha comentado el comportamiento de este tipo de aparamenta de manera conjunta, es decir, las características comunes a los tres contactores. Por lo tanto, en las próximas líneas se van a resumir aspectos individuales de los contactores ensayados en vacío, donde una primera visualización se ha mostrado en la tabla 2.11

El contactor Mitsubishi es el más sensible de los tres contactores ensayados. Específicamente, ante huecos de tensión con reducido ángulo de inicio de la onda, el contactor Mitsubishi dispara ante duraciones de hueco considerablemente inferiores que los otros dos contactores ensayados. Por ejemplo, para una interrupción del suministro con ángulo inicial de $0^{\circ}$ sólo soporta $45 \mathrm{~ms}$; mientras que el contactor Schneider no abre sus contactos hasta que la duración de la dicha perturbación no supera $57 \mathrm{~ms}$, y el contactor Tele- 
mecanique hasta superar $64 \mathrm{~ms}$. Para mayores ángulos de inicio de la onda de tensión, todos los contactores disminuyen sus tiempos ante huecos menos profundos, aunque continúa siendo el contactor Mitsubishi el que presenta el menor tiempo.

Por otra parte, ante huecos de larga duración los contactores Mitsubishi y Schneider se desconectan para profundidades del $50 \%$; mientras que para producir el disparo del contactor Telemecanique es necesario una profundidad del $60 \%$. Sin embargo, para elevados ángulos de inicio de la onda de tensión, se observa que los tres contactores soportarían duraciones de hueco similares ante elevadas profundidades del hueco, tal y como se observa en el valor de la variable $t_{\max _{90} 0}$ en la tabla 2.11 .

Por último, es importante observar que las bandas de dispersión obtenidas en el contactor Mitsubishi son de mayor extensión que las que existen en los otros dos.

\subsubsection{Ensayos con carga resistiva}

Con el objeto de observar la influencia del grado de carga sobre la respuesta de los contactores, se han realizado tres ensayos sobre el contactor Telemecanique, variando tanto el ángulo de inicio de la onda de tensión como el valor de la carga resistiva conectada, figura 2.18. Concretamente, la figura 2.18a muestra la curva de tolerancia del contactor alimentando una carga resistiva por fase (las especificaciones de la carga se encuentran en el apartado 2.3.2.1, cuando el ángulo de inicio de la onda de tensión es igual a $0^{\circ}$. En la figura $2.18 \mathrm{~b}$ se ensaya el contactor alimentando dos resistencias en serie bajo $90^{\circ}$; mientras que en la figura $2.18 \mathrm{c}$ se aumenta la intensidad que atraviesa el contactor mediante la conexión en estrella de dos resistencias en paralelo para un ángulo de inicio de la onda igual a $60^{\circ}$.

Comparando las figuras 2.18a, 2.18b y 2.18c con sus respectivas en vacío, figuras 2.16a, 2.16g y 2.16e, se puede concluir que la morfología de las curvas de tolerancia no varía en función de la intensidad que atraviese el contactor cuando éste se encuentra conectado a una carga resistiva. Además, los valores límites de tensión y/o tiempo que podría soportar el contactor tampoco resultan alterados.

Con el objeto de confirmar este resultado, también se han ensayado los otros dos contactores bajo diferentes condiciones de carga resistiva y ángulo de inicio del hueco de tensión; aunque no se han mostrado más imágenes, dado que se obtiene la misma conclusión.

\subsubsection{Ensayos con carga inductiva}

Una vez observado que la respuesta de los contactores ante huecos de tensión e interrupciones no resulta modificada cuando se encuentran suministrando energía eléctrica a una carga resistiva, se va a analizar si la variación 


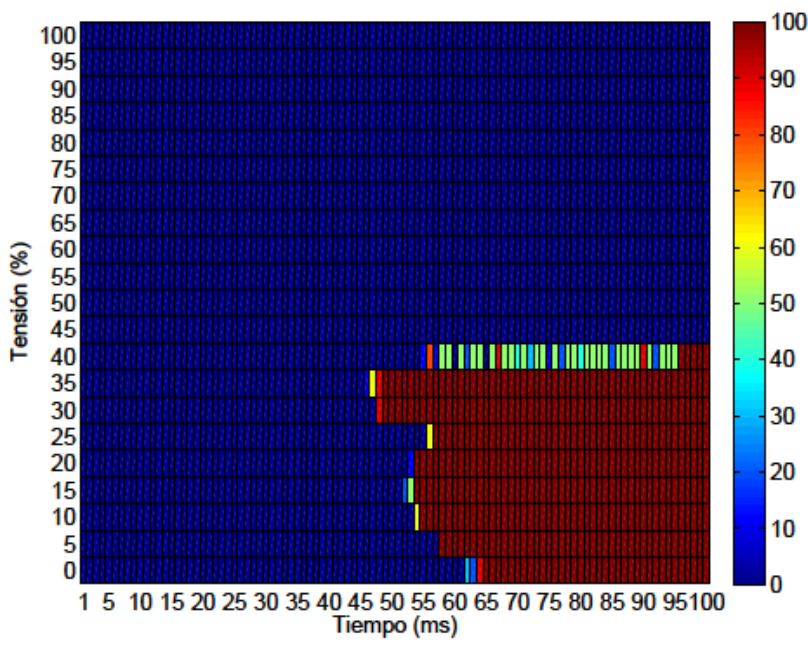

(a) Probabilidad de fallo bajo una carga resistiva para $0^{\circ}$

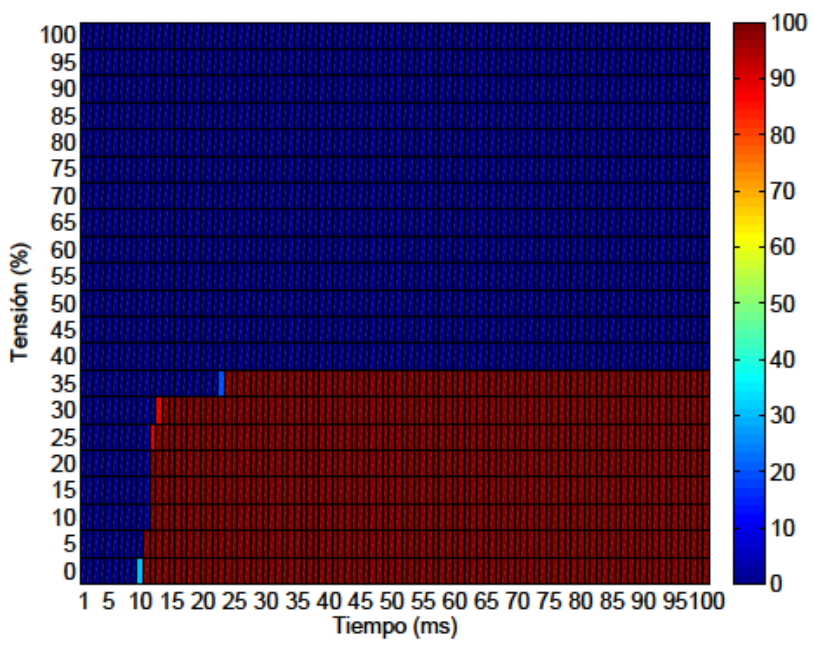

(b) Probabilidad de fallo bajo carga resistiva en serie para $90^{\circ}$.

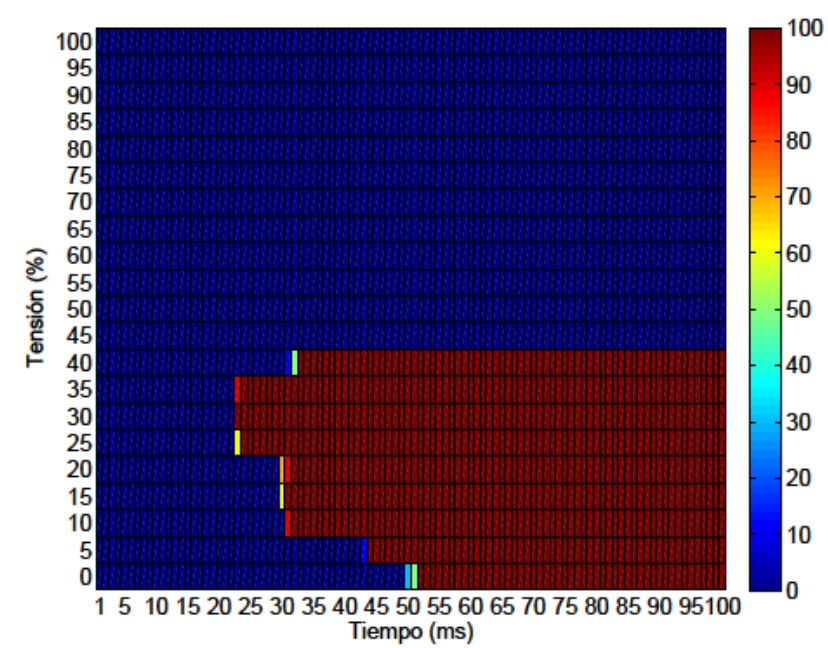

(c) Probabilidad de fallo bajo carga resistiva en paralelo para $60^{\circ}$.

Figura 2.18: Curvas de tolerancia del contactor Telemecanique alimentando diversas cargas resistivas. 
del tipo de carga influye sobre dicha respuesta. Por lo tanto, continuando con el mismo procedimiento que se ha presentado para la carga resistiva, los experimentos llevados a cabo bajo la carga inductiva descrita en el apartado 2.3.2.1 incluyen tanto la alimentación de una carga por fase como de dos en paralelo.

De esta manera, las figuras $2.19,2.20$ y 2.21 muestran las curvas de tolerancia de los contactores Schneider, Telemecanique y Mitsubishi, respectivamente, cuando se encuentran alimentando una carga inductiva, en función del ángulo de inicio de la onda de tensión. A la vista de estas tres figuras, se puede observar que existen notables diferencias en relación con el comportamiento de los contactores ante huecos de tensión e interrupciones, en comparación con las obtenidas en vacío, figuras 2.15, 2.16 y 2.17.

Por un lado, aunque los contactores se encuentren suministrando energía a una carga inductiva, se continúa respetando la forma de la curva de tolerancia obtenida en vacío —o bajo carga resistiva- Es decir, a menor ángulo de inicio de la onda de tensión, los tres contactores ensayados presentan una mayor capacidad para soportar huecos profundos de larga duración; mientras que conforme aumenta dicho ángulo se reduce drásticamente la duración que los contactores pueden soportar ante un hueco de tensión de elevada profundidad.

Por el contrario, una de las primeras diferencias relevantes que se ha encontrado en los tres contactores ensayados es la disminución de la zona de dispersión cuando éstos alimentan una carga inductiva. Concretamente, en el caso del contactor Telemecanique, este resultado se comprueba gráficamente observando las figuras $2.16 \mathrm{~b}$ y $2.20 \mathrm{~b}$ para tensiones residuales con valores entre el 35\%-40\% de la tensión nominal. Por lo tanto, la respuesta de los contactores se puede predecir con mayor precisión cuando éstos suministran energía a una carga inductiva.

En cuanto a los tiempos de disparo, se puede observar que éstos se reducen considerablemente para ángulos de inicio de la onda de tensión comprendidos entre $0^{\circ}$ y $60^{\circ}$ cuando la tensión residual del hueco de tensión se sitúa entre el $0 \%$ y el $20 \%$ de la tensión nominal; ya que para mayores tensiones residuales el tiempo de disparo es similar al que presenta en vacío. Concretamente, el contactor Telemecanique es el que más aumenta su sensibilidad dentro del rango anterior, ya que la diferencia asciende hasta $9 \mathrm{~ms}$ en el caso de $30^{\circ}$ y $100 \%$ de profundidad. Para mayores ángulos de inicio de la onda de tensión, entre $75^{\circ}$ y $90^{\circ}$, se observa que los contactores ensayados no resultan afectados de manera significativa por la presencia de la carga inductiva presentando, por lo tanto, un comportamiento similar al de vacío.

En relación con la capacidad para soportar huecos de tensión de larga duración, no se ha encontrado una respuesta uniforme en los tres contactores ensayados. Por ejemplo, el contactor Telemecanique no varía la tensión residual mínima tolerable en ninguno de los ángulos de inicio ensayados ba- 


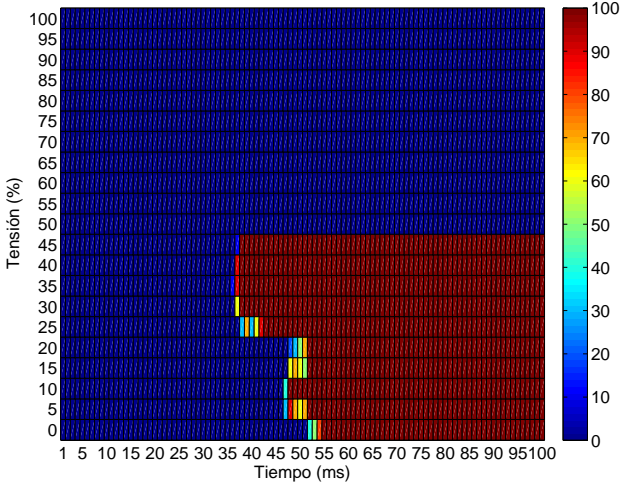

(a) Probabilidad de fallo para $0^{\circ}$.

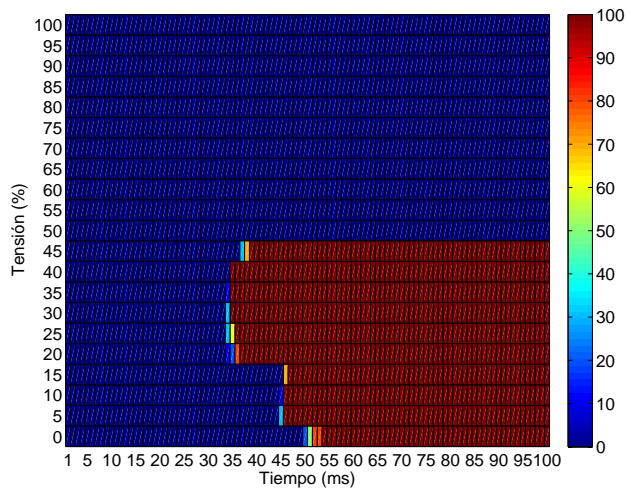

(c) Probabilidad de fallo para $30^{\circ}$.

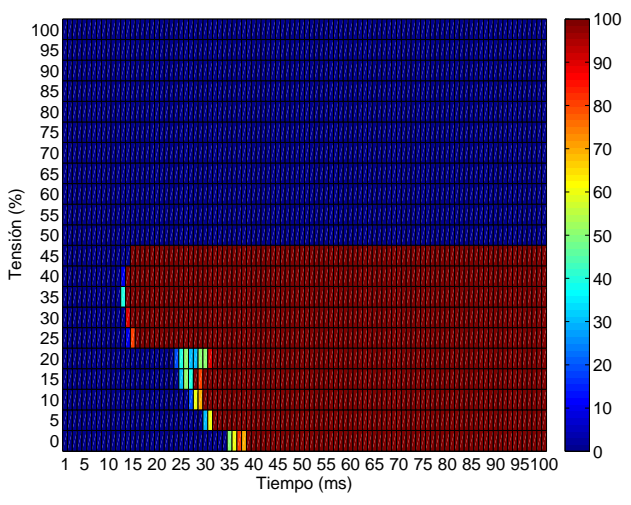

(e) Probabilidad de fallo para $60^{\circ}$.

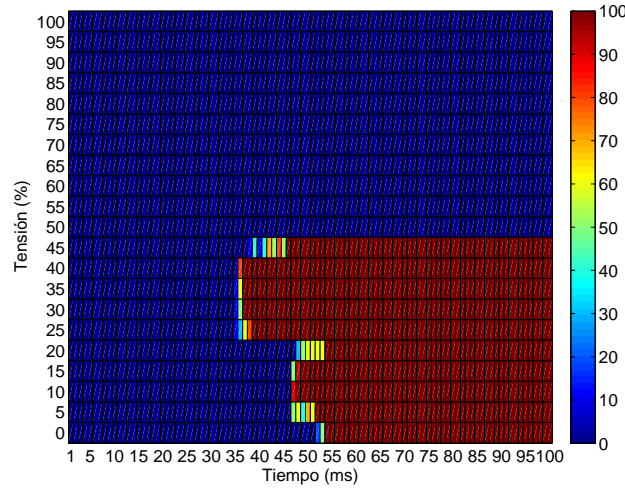

(b) Probabilidad de fallo para $15^{\circ}$.

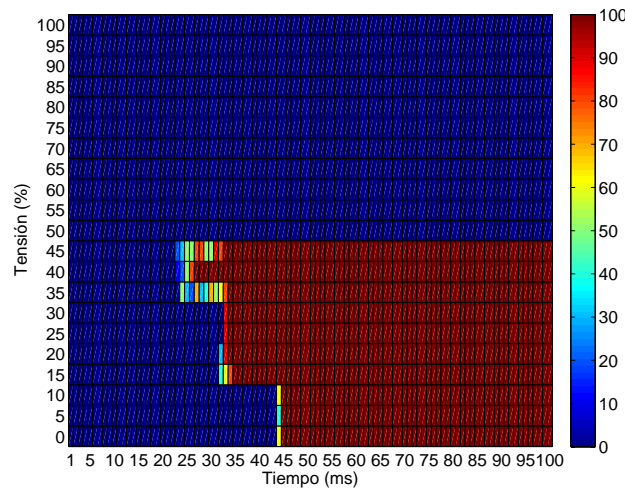

(d) Probabilidad de fallo para $45^{\circ}$.

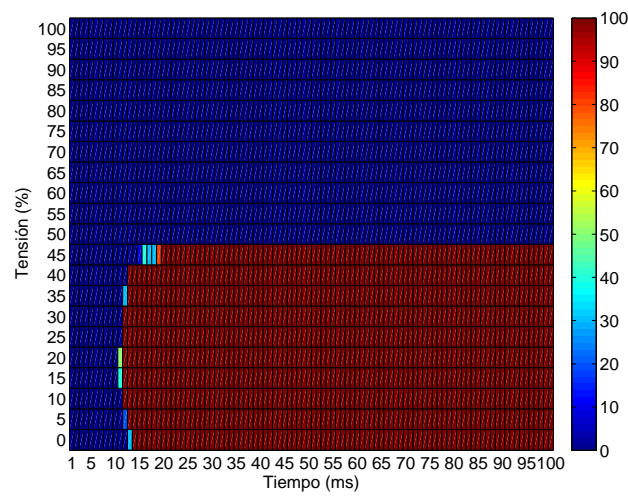

(f) Probabilidad de fallo para $75^{\circ}$

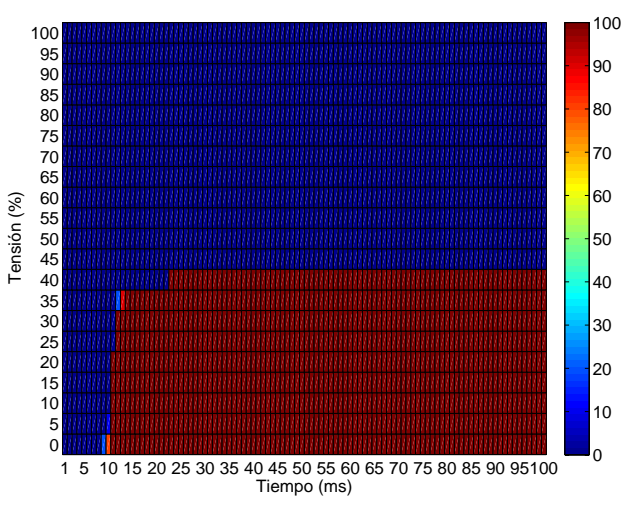

(g) Probabilidad de fallo para $90^{\circ}$.

Figura 2.19: Curvas de tolerancia del contactor Schneider. Carga inductiva. 


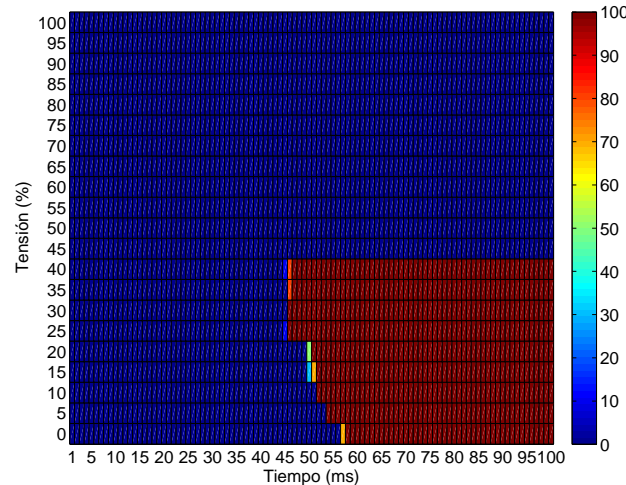

(a) Probabilidad de fallo para $0^{\circ}$.

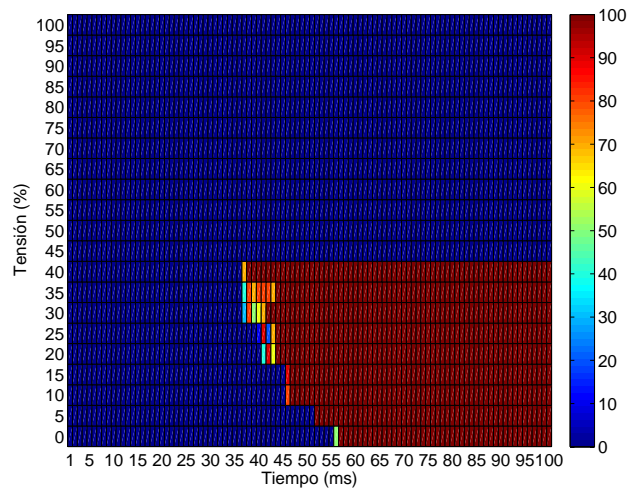

(c) Probabilidad de fallo para $30^{\circ}$.

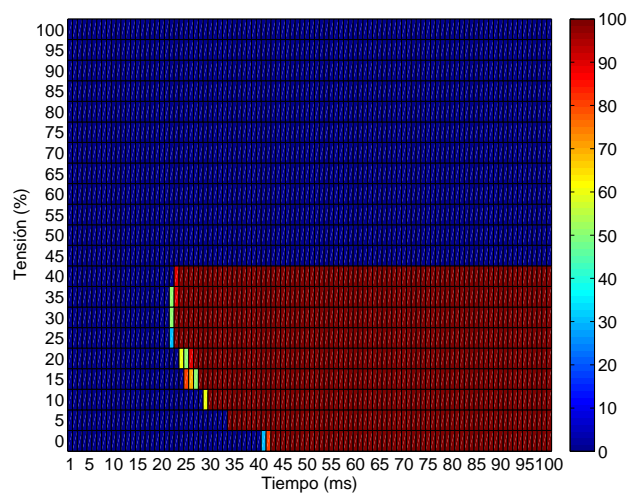

(e) Probabilidad de fallo para $60^{\circ}$.

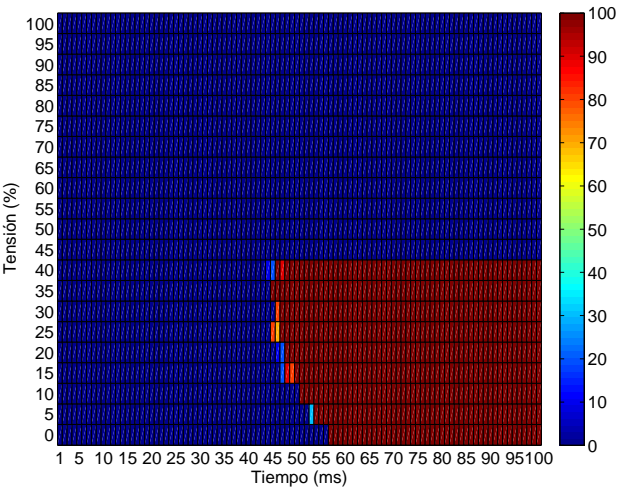

(b) Probabilidad de fallo para $15^{\circ}$.

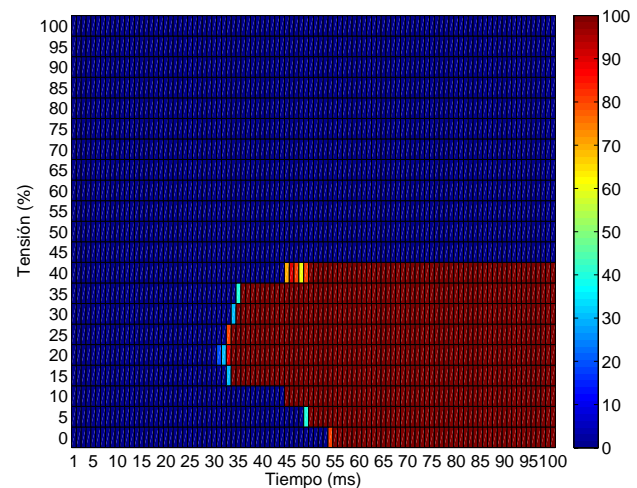

(d) Probabilidad de fallo para $45^{\circ}$.

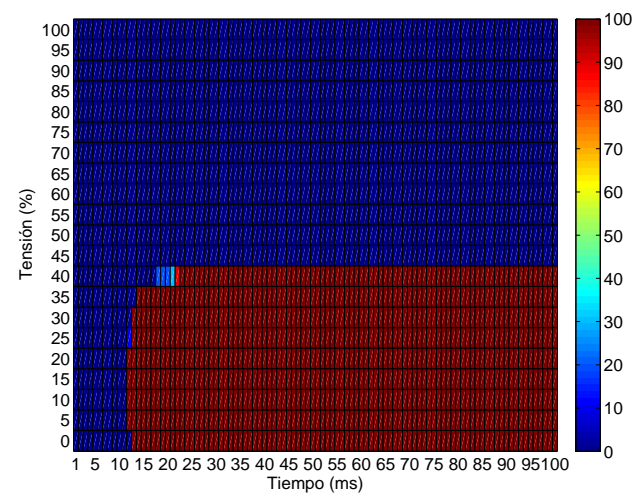

(f) Probabilidad de fallo para $75^{\circ}$.

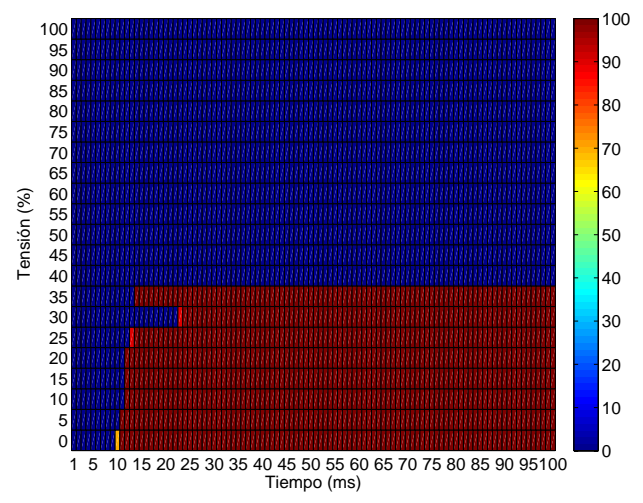

(g) Probabilidad de fallo para $90^{\circ}$.

Figura 2.20: Curvas de tolerancia del contactor Telemecanique. Carga inductiva. 


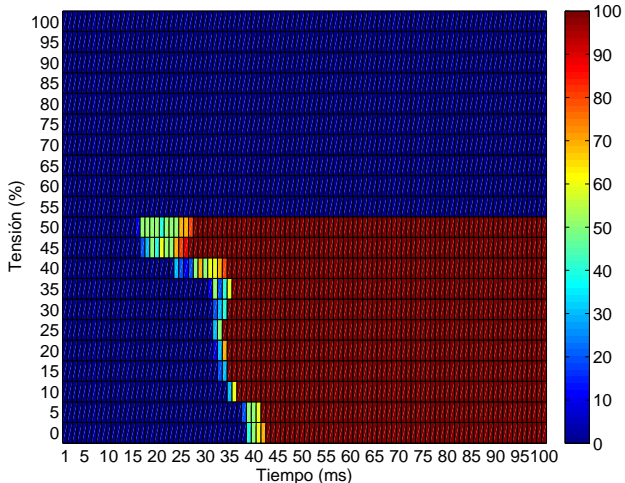

(a) Probabilidad de fallo para $0^{\circ}$.

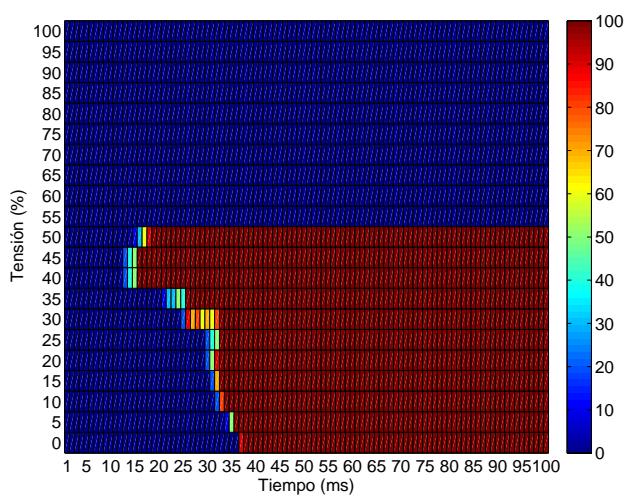

(c) Probabilidad de fallo para $30^{\circ}$.

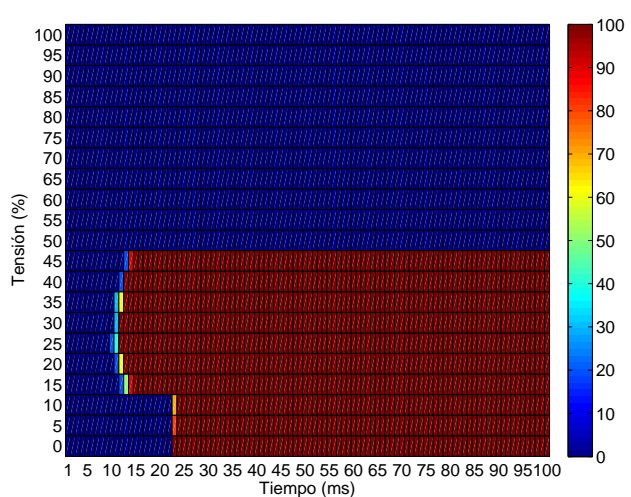

(e) Probabilidad de fallo para $60^{\circ}$.

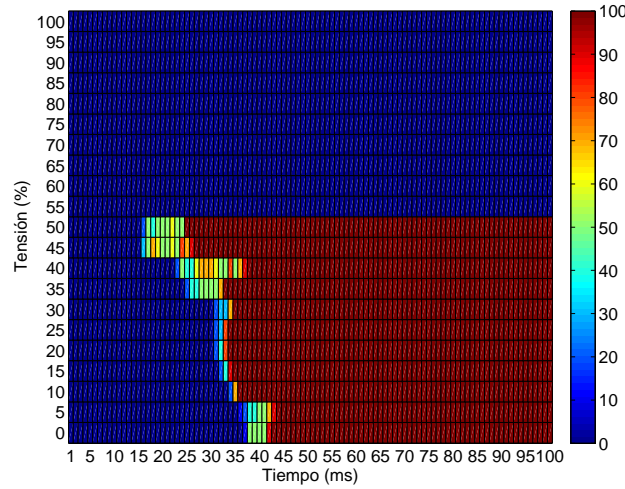

(b) Probabilidad de fallo para $15^{\circ}$

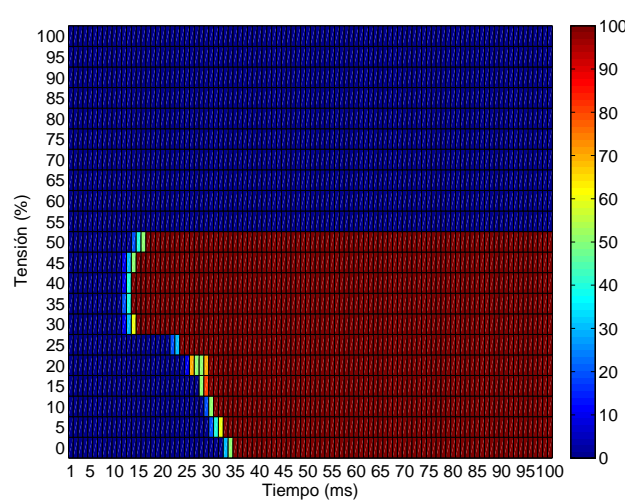

(d) Probabilidad de fallo para $45^{\circ}$.

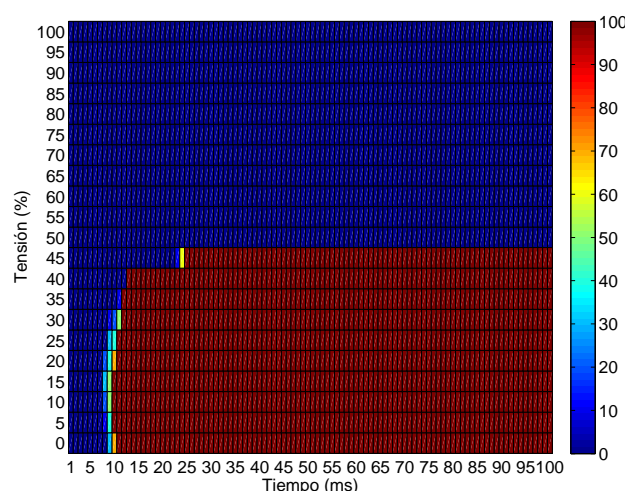

(f) Probabilidad de fallo para $75^{\circ}$.

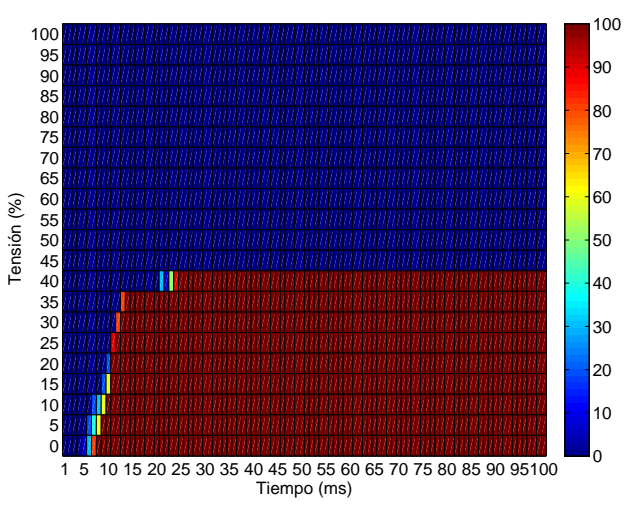

(g) Probabilidad de fallo para $90^{\circ}$.

Figura 2.21: Curvas de tolerancia del contactor Mitsubishi. Carga inductiva. 
jo carga inductiva en comparación con su comportamiento en vacío o bajo carga resistiva. Sin embargo, el contactor Mitsubishi es capaz de soportar huecos de tensión un $5 \%$ más profundos para ángulos de inicio de $60^{\circ} \mathrm{y}$ $90^{\circ}$ cuando alimenta a la carga inductiva. De manera similar, el contactor Schneider puede tolerar huecos de tensión un $5 \%$ más profundos cuando el ángulo de inicio del hueco es de $0^{\circ}$ ó $15^{\circ}$; mientras que para un ángulo de $75^{\circ}$ su tolerancia se reduce un $5 \%$.

Para intentar obtener unos resultados más concluyentes, se han realizado los mismos ensayos bajo carga inductiva aunque, en esta ocasión, duplicando la intensidad que atraviesa cada contactor mediante la conexión en paralelo de dos reactancias por fase, figuras 2.22, 2.23 y 2.24. De esta manera, la intensidad que atraviesa al contactor se encuentra próxima a su valor nominal en categoría AC-3 (ver tabla 2.2.

Analizando con atención las figuras 2.22, 2.23 y 2.24, se pueden extraer similares conclusiones a las que se obtuvieron cuando cada contactor sólo estaba conectado a una carga inductiva, figuras 2.19, 2.20 y 2.21 .

Se puede observar que el aumento de la intensidad inductiva resulta ser inversamente proporcional al tiempo de disparo del contactor para profundidades del hueco superiores al $80 \%$. Por ejemplo, en el caso del contactor Mitsubishi, al conectar dos bobinas en paralelo por fase el tiempo se reduce alrededor de $8 \mathrm{~ms}$; lo que implica una disminución del tiempo de disparo de este contactor próxima a $15 \mathrm{~ms}$ con respecto a su ensayo en vacío. De manera similar a lo que se experimentó cuando sólo estaba conectada una bobina por fase, para tensiones residuales superiores al $20 \%$ de la tensión nominal, la modificación del tiempo de disparo resulta despreciable. También se vuelve a cumplir que, para los ángulos de inicio de $75^{\circ}$ y $90^{\circ}$, el comportamiento de los contactores no resulta modificado por el tipo de carga inductiva que tengan conectada.

Además, se repite el fenómeno de disminución de la zona de dispersión de los contactores. Por ejemplo, en la figura $2.22 \mathrm{~b}$ se puede comprobar la relevante reducción de las bandas de dispersión con respecto a las figuras $2.19 \mathrm{~b}$ - sólo una carga inductiva-y 2.15b-en vacío-.

En relación con la capacidad para soportar huecos de tensión de larga duración, los ensayos bajo doble carga inductiva muestran exactamente el mismo valor de tensión residual mínima tolerable que bajo una carga inductiva por fase. Por lo tanto, no se está en condiciones de afirmar que se modifique dicho valor límite en función de la carga que esté alimentando el contactor.

También es importante señalar que, con el objeto de comprobar la coherencia de los resultados obtenidos, también se han realizado ensayos con los contactores alimentando a dos cargas inductivas en serie. De esta manera, la intensidad inductiva se reduce a la mitad con respecto a cuando se suministra energía a una carga. Los resultados que se han obtenido se encuentran 


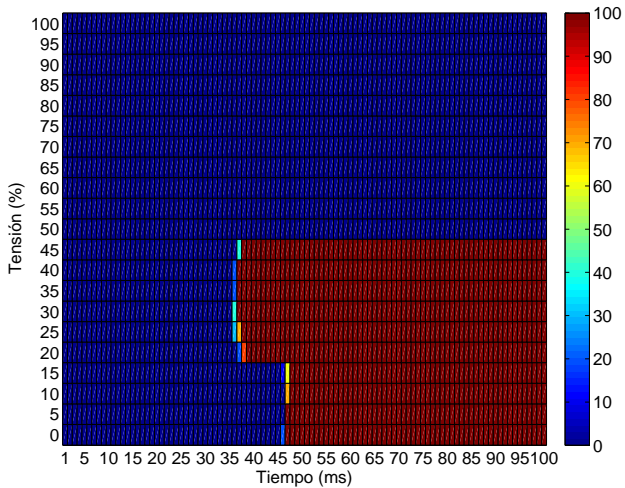

(a) Probabilidad de fallo para $0^{\circ}$.

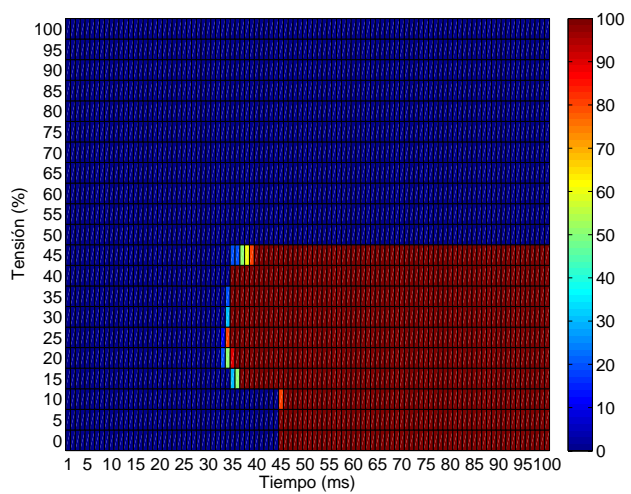

(c) Probabilidad de fallo para $30^{\circ}$.

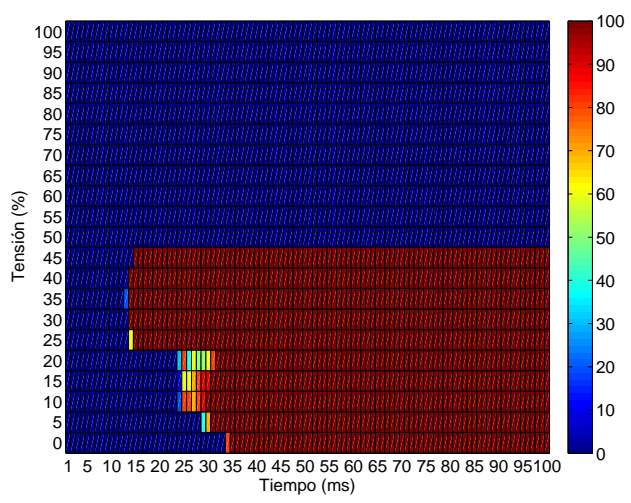

(e) Probabilidad de fallo para $60^{\circ}$.

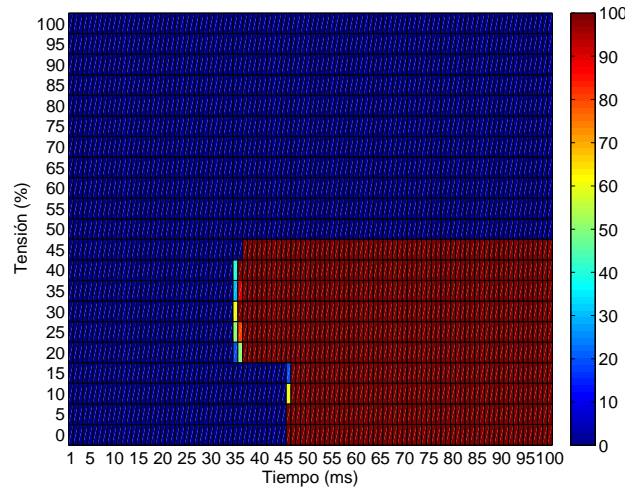

(b) Probabilidad de fallo para $15^{\circ}$.

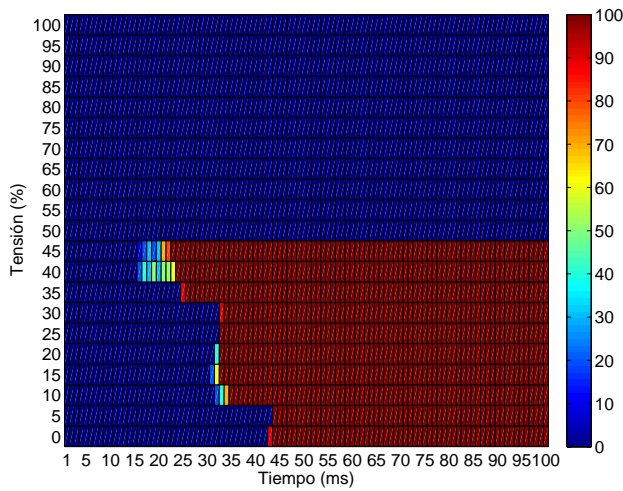

(d) Probabilidad de fallo para $45^{\circ}$.

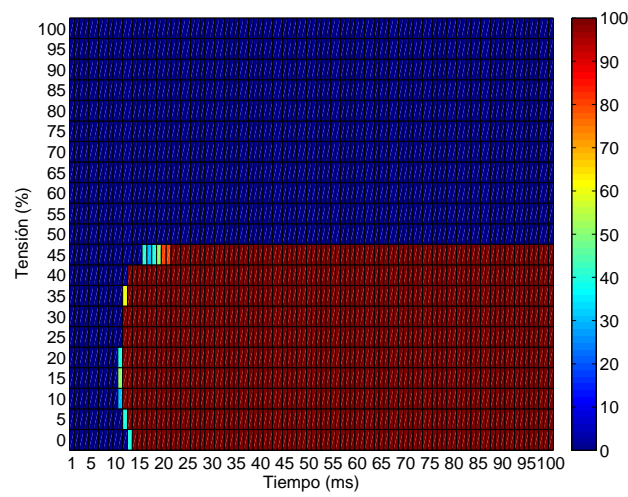

(f) Probabilidad de fallo para $75^{\circ}$.

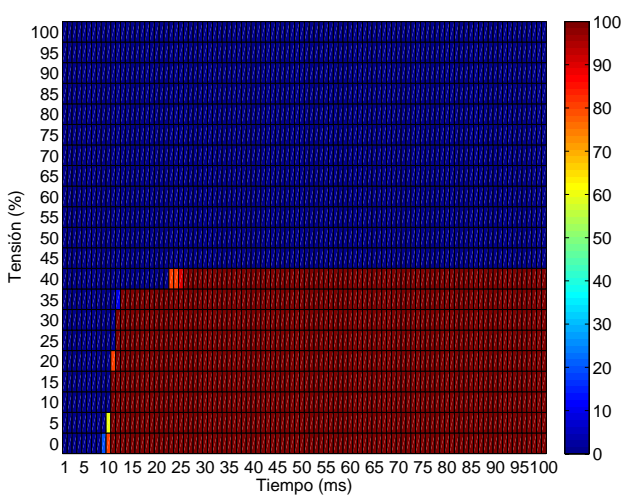

(g) Probabilidad de fallo para $90^{\circ}$.

Figura 2.22: Curvas de tolerancia del contactor Schneider. Carga inductiva en paralelo. 


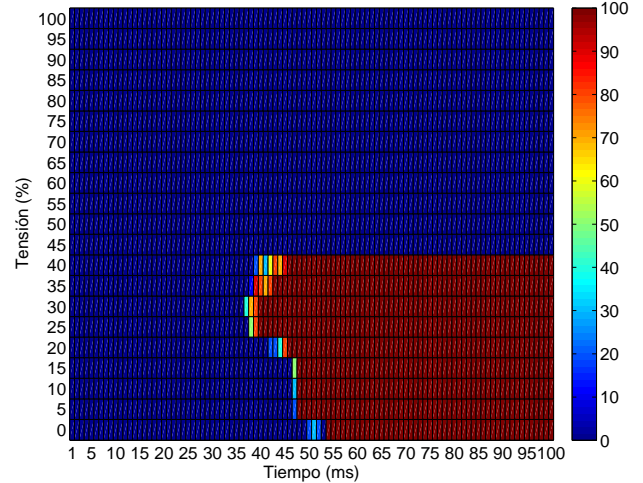

(a) Probabilidad de fallo para $0^{\circ}$.

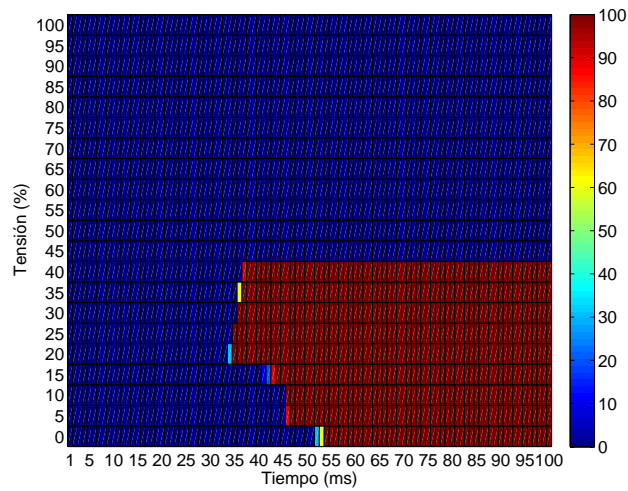

(c) Probabilidad de fallo para $30^{\circ}$.

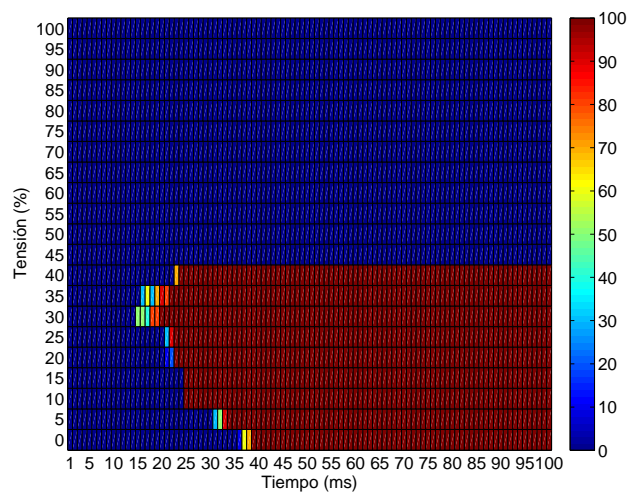

(e) Probabilidad de fallo para $60^{\circ}$.

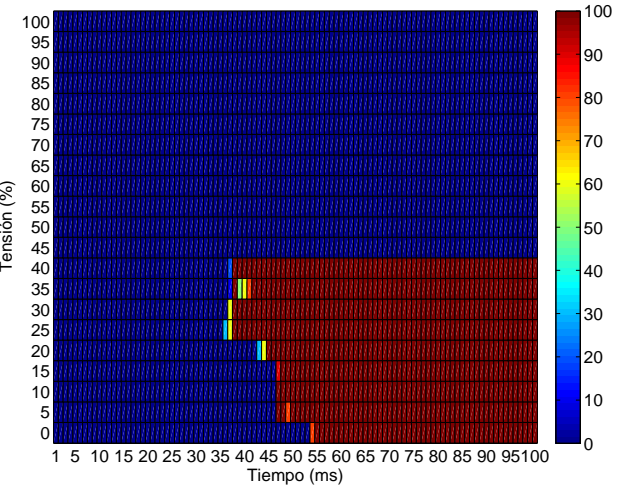

(b) Probabilidad de fallo para $15^{\circ}$.

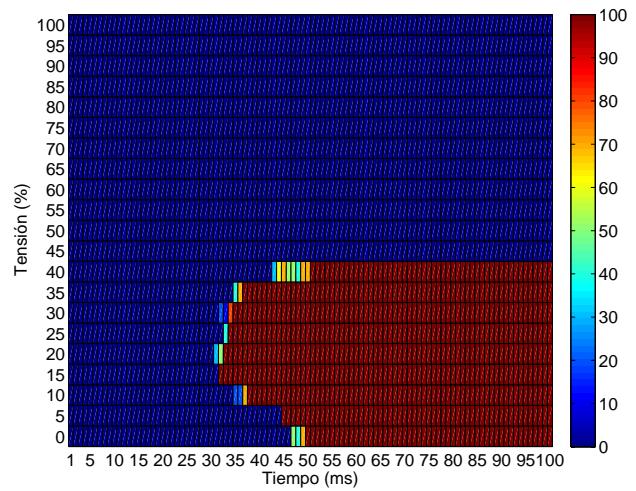

(d) Probabilidad de fallo para $45^{\circ}$.

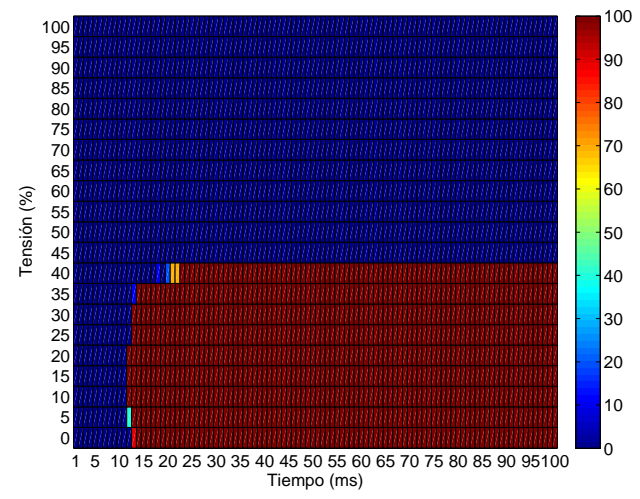

(f) Probabilidad de fallo para $75^{\circ}$.

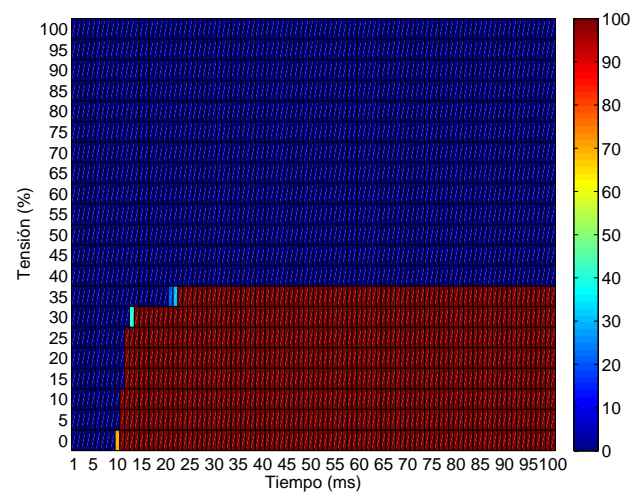

(g) Probabilidad de fallo para $90^{\circ}$.

Figura 2.23: Curvas de tolerancia del contactor Telemecanique. Carga inductiva en paralelo. 


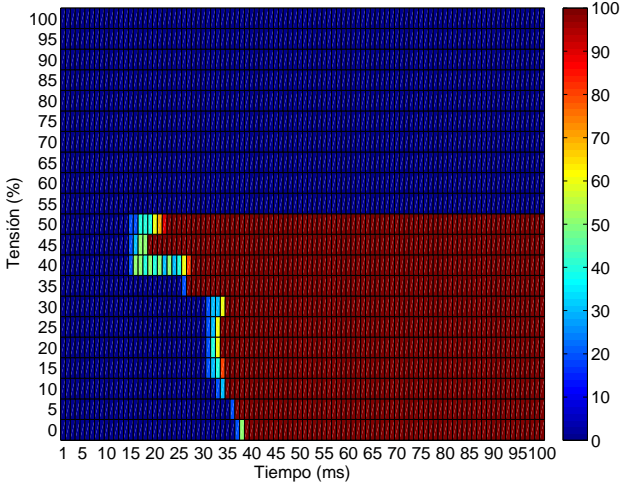

(a) Probabilidad de fallo para $0^{\circ}$.

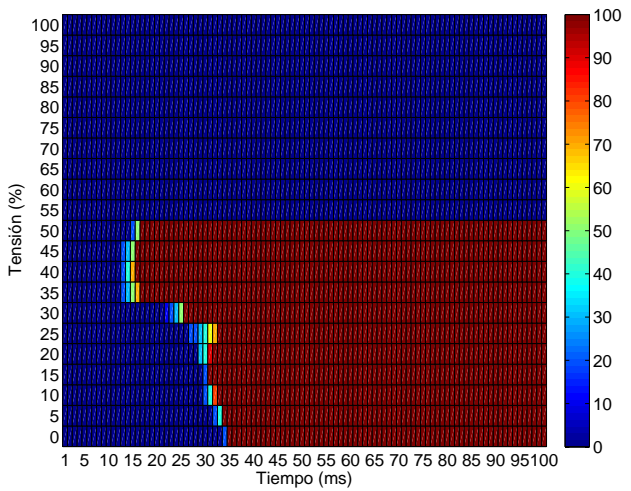

(c) Probabilidad de fallo para $30^{\circ}$.

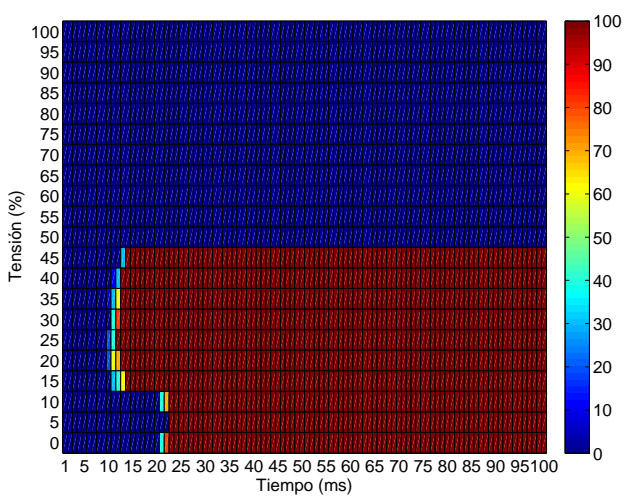

(e) Probabilidad de fallo para $60^{\circ}$.

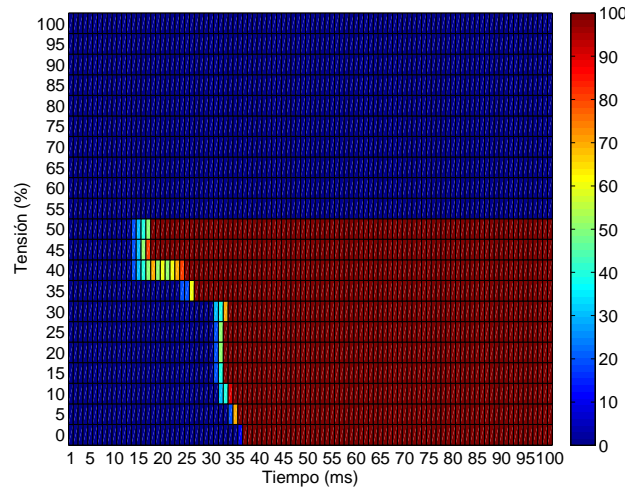

(b) Probabilidad de fallo para $15^{\circ}$.

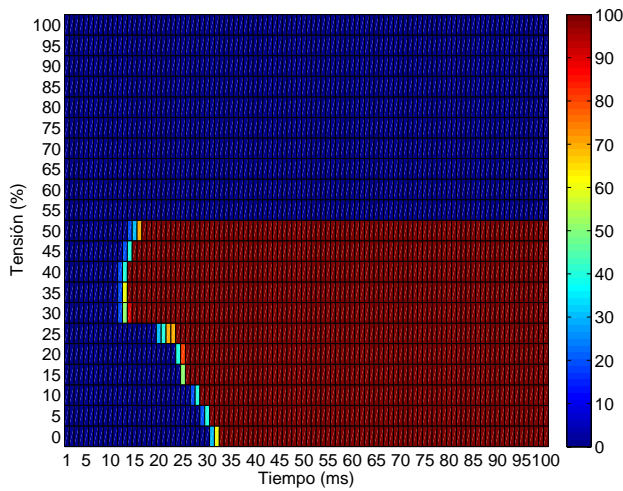

(d) Probabilidad de fallo para $45^{\circ}$.

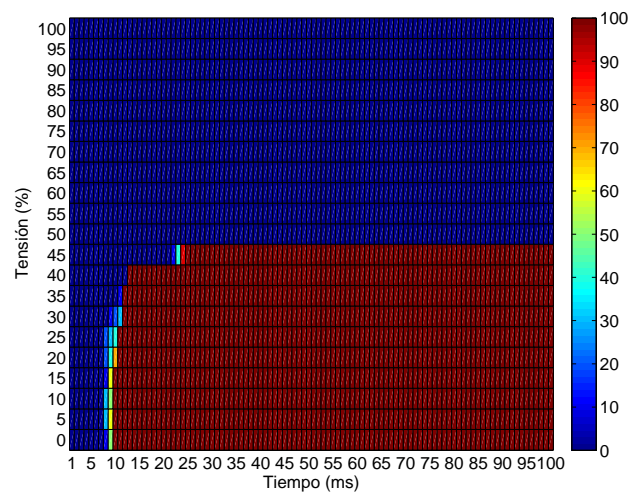

(f) Probabilidad de fallo para $75^{\circ}$.

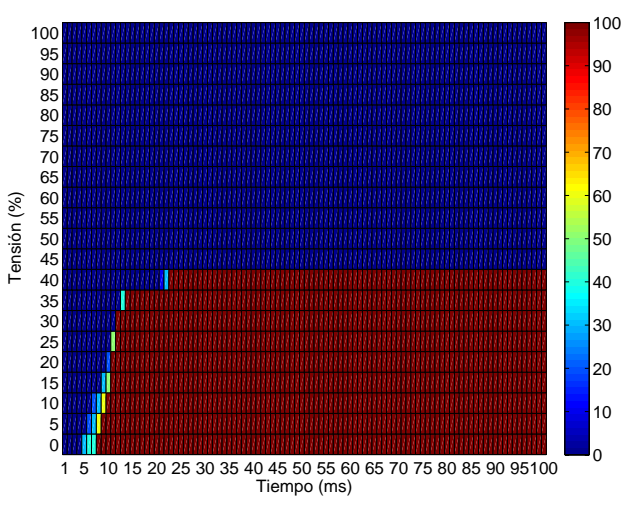

(g) Probabilidad de fallo para $90^{\circ}$.

Figura 2.24: Curvas de tolerancia del contactor Mitsubishi. Carga inductiva en paralelo. 
en línea con los que se han presentado anteriormente.

\subsubsection{Ensayos con carga capacitiva}

Una vez analizada la variación de la respuesta de este tipo de aparamenta eléctrica cuando se encuentra alimentando a una carga inductiva, con el interés de completar el análisis del comportamiento de este tipo de aparamenta eléctrica ante huecos de tensión e interrupciones, las figuras 2.25, 2.26 y 2.27 muestran las curvas de tolerancia de los tres contactores ensayados cuando alimentan al banco de condensadores descrito en el apartado 2.3.2.1.

La primera deducción que se puede extraer de las figuras 2.25, 2.26 y 2.27 , con respecto a cuando los contactores estaban en vacío, es que el comportamiento de los contactores frente a huecos de tensión e interrupciones es modificado de manera significativa cuando se encuentran alimentando a una carga capacitiva.

Se observa que, en esta ocasión, la forma de las curvas de tolerancia es muy similar en cualquiera de los ángulos de inicio de la onda de tensión ensayados. En los tres contactores ensayados, para ángulos de inicio superiores a $60^{\circ}$, la curva de tolerancia obtenida no tiende a ser rectangular, fenómeno que sí ocurría cuando se ensayaron en vacío, bajo carga resistiva y bajo carga inductiva.

La situación anterior ocasiona que los tiempos de disparo sean muy similares independientemente del ángulo de inicio de la onda de tensión. Por ejemplo, en la figura 2.27 se observa que el contactor Mitsubishi puede soportar interrupciones del suministro de una duración comprendida entre 40 ms$45 \mathrm{~ms}$ sea cual sea el ángulo de inicio de la onda de tensión.

Por lo tanto, la respuesta de cada contactor ante huecos de tensión e interrupciones cuando se encuentran conectados a una carga capacitiva se puede resumir en una sola curva de tolerancia.

\subsubsection{Influencia de la temperatura en la respuesta de contac- tores ante huecos de tensión e interrupciones}

Tal y como se indicó en el apartado 2.3.3, todos los ensayos que se han presentado sobre contactores han sido realizados bajo una temperatura de servicio constante, en torno a $45{ }^{\circ} \mathrm{C}$, medida sobre la carcasa del contactor. De esta manera, se ha simulando la operación de este tipo de aparamenta en cualquier instalación en que se encuentre funcionando de manera continua.

Sin embargo, también se ha investigado sobre la respuesta de este equipamiento cuando no ha alcanzado la temperatura de operación constante. Con este interés, la figura 2.28 resume los experimentos efectuados sobre el contactor Schneider para un ángulo de inicio de la onda de tensión igual a $0^{\circ}$. Sobre dicha figura, en color azul se ha trazado el perfil de tolerancia cuando la temperatura de la bobina se corresponde con un valor "caliente" 


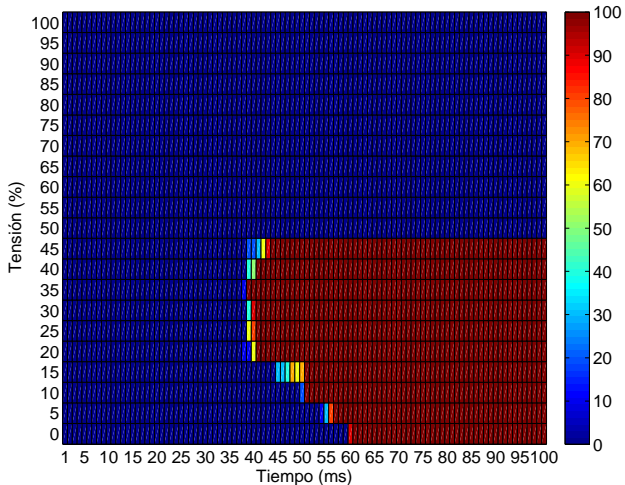

(a) Probabilidad de fallo para $0^{\circ}$.

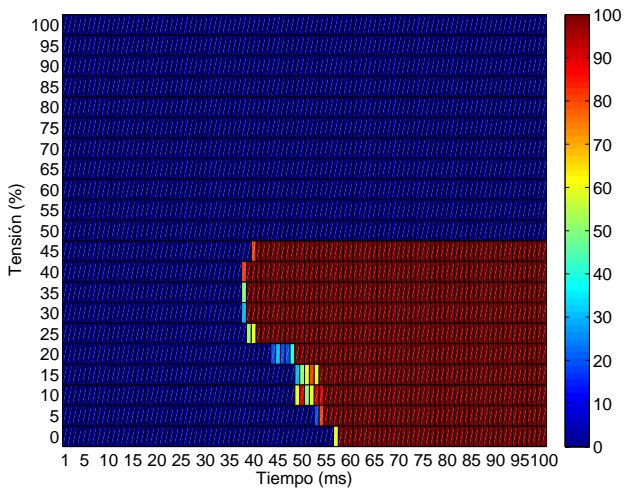

(c) Probabilidad de fallo para $30^{\circ}$.

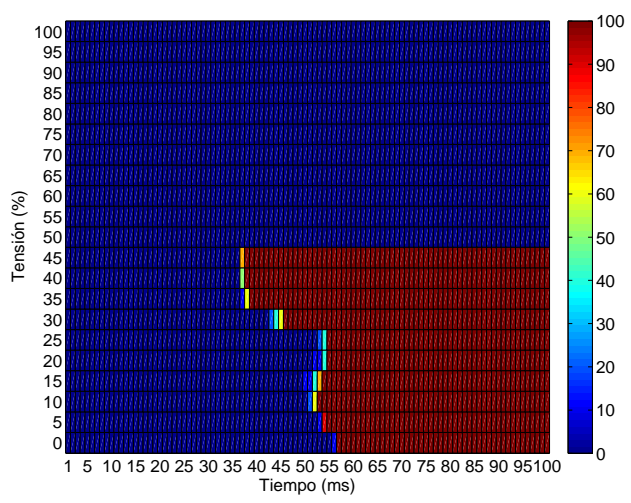

(e) Probabilidad de fallo para $60^{\circ}$.

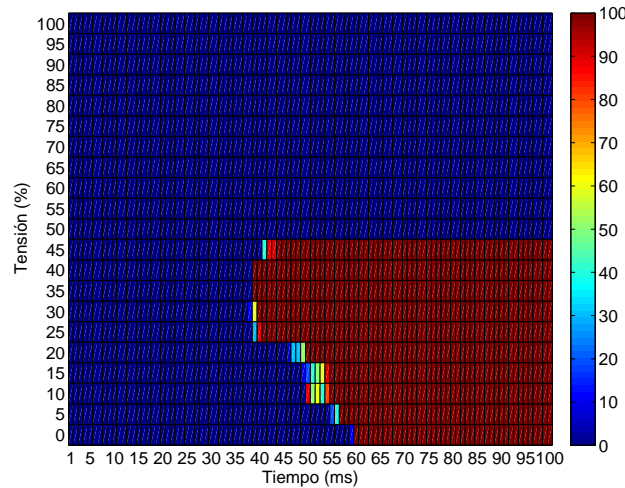

(b) Probabilidad de fallo para $15^{\circ}$.

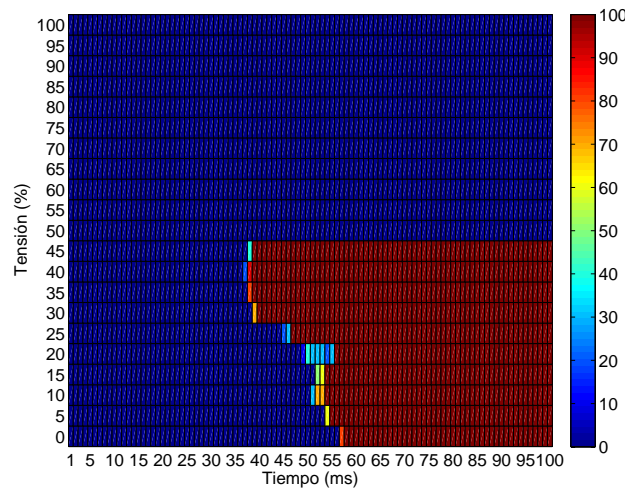

(d) Probabilidad de fallo para $45^{\circ}$.

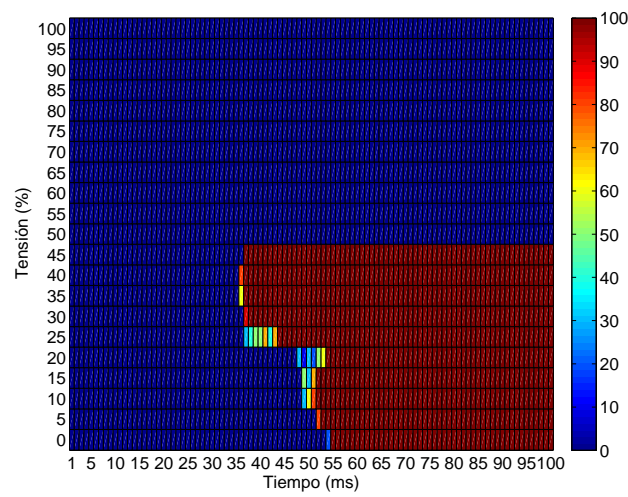

(f) Probabilidad de fallo para $75^{\circ}$

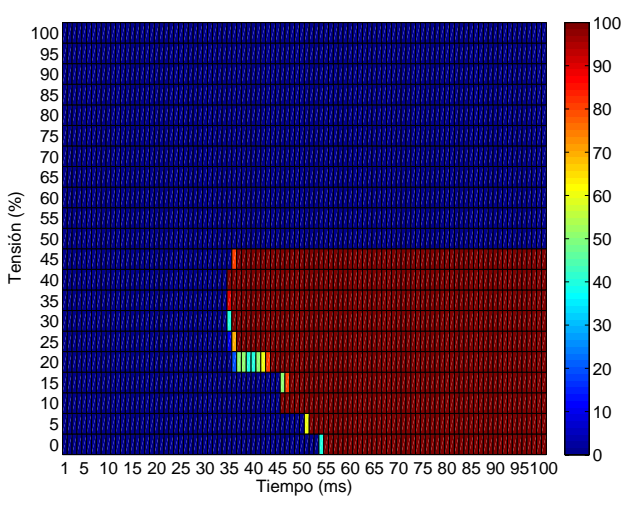

(g) Probabilidad de fallo para $90^{\circ}$.

Figura 2.25: Curvas de tolerancia del contactor Schneider. Carga capacitiva. 


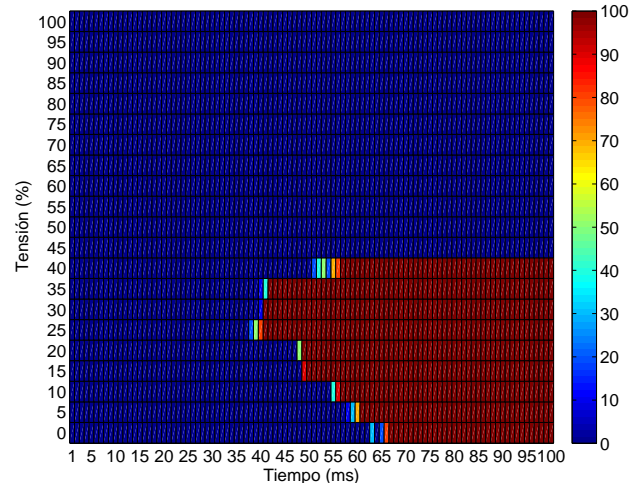

(a) Probabilidad de fallo para $0^{\circ}$.

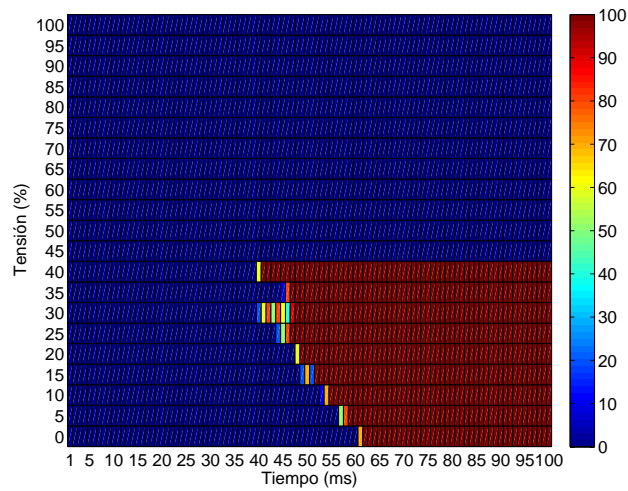

(c) Probabilidad de fallo para $30^{\circ}$.

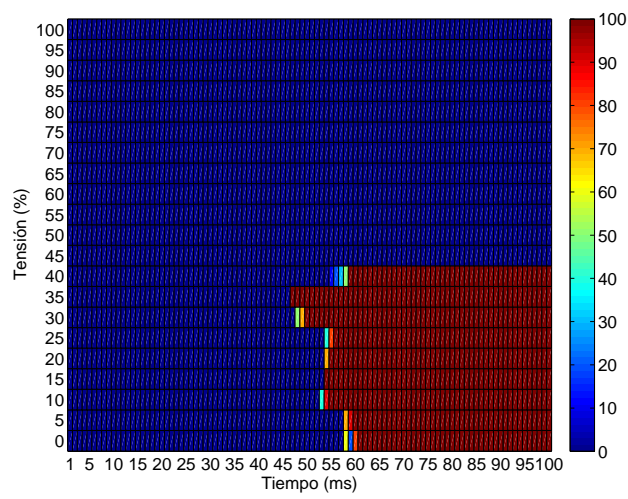

(e) Probabilidad de fallo para $60^{\circ}$.

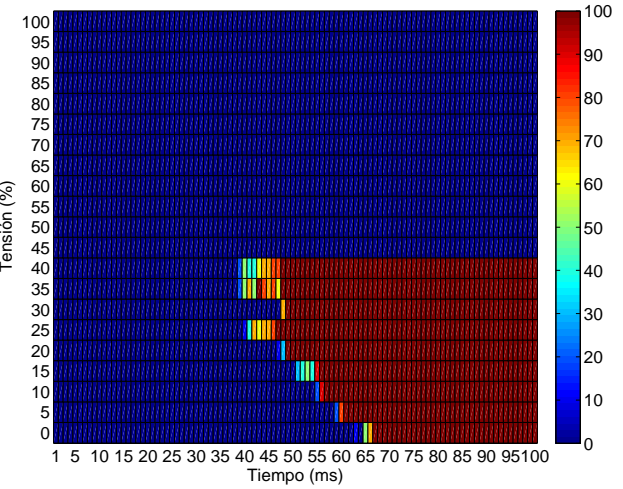

(b) Probabilidad de fallo para $15^{\circ}$.

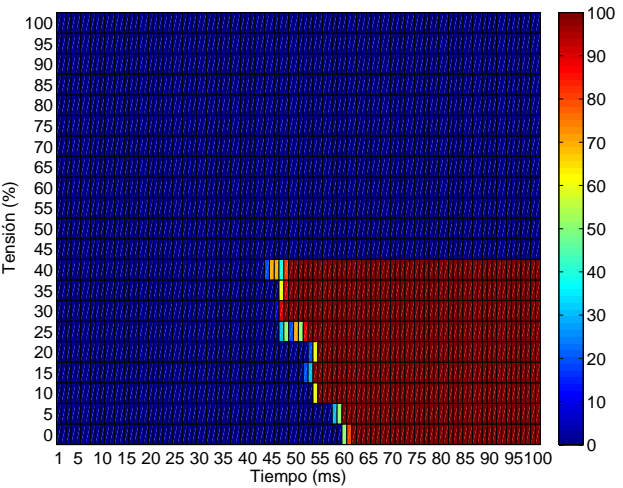

(d) Probabilidad de fallo para $45^{\circ}$.

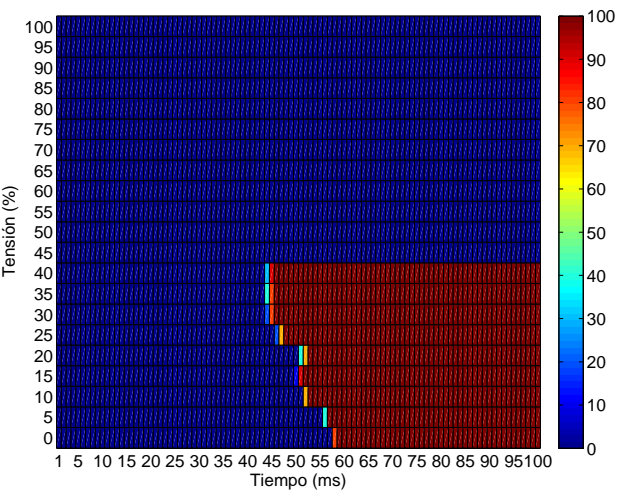

(f) Probabilidad de fallo para $75^{\circ}$.

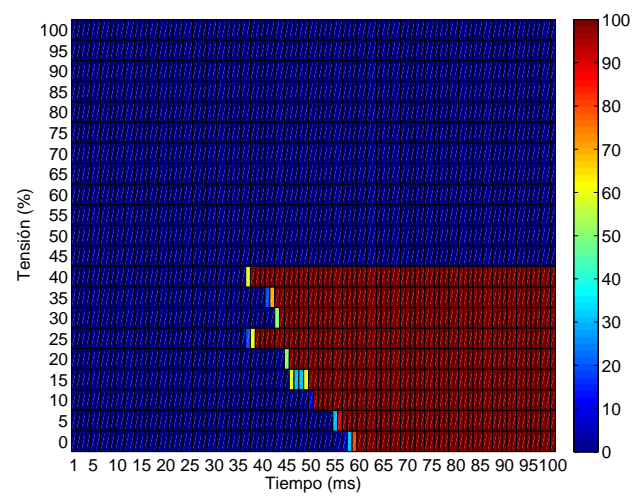

(g) Probabilidad de fallo para $90^{\circ}$.

Figura 2.26: Curvas de tolerancia del contactor Telemecanique. Carga capacitiva. 


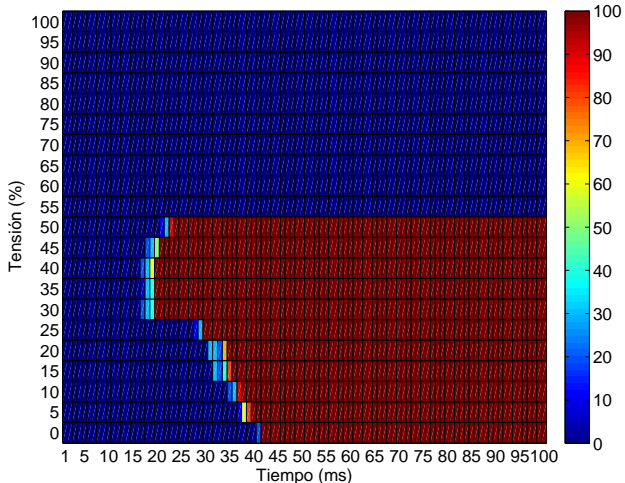

(a) Probabilidad de fallo para $0^{\circ}$.

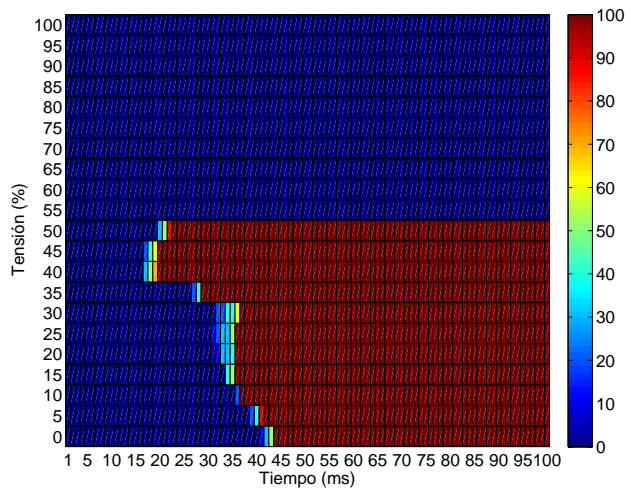

(c) Probabilidad de fallo para $30^{\circ}$.

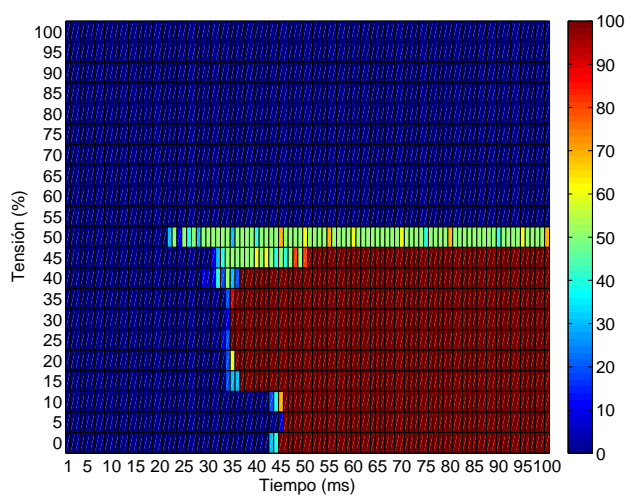

(e) Probabilidad de fallo para $60^{\circ}$.

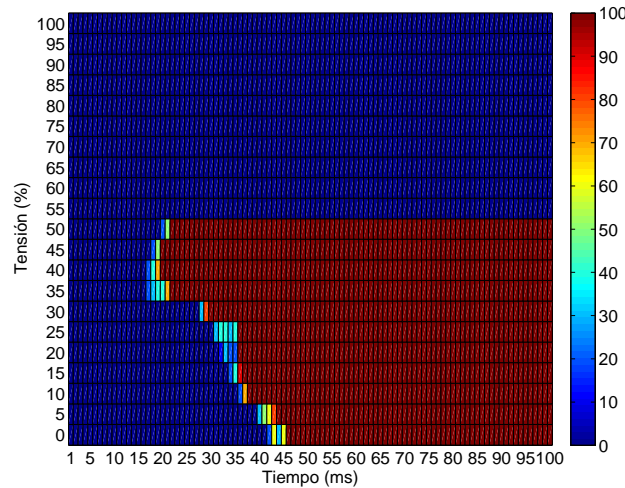

(b) Probabilidad de fallo para $15^{\circ}$

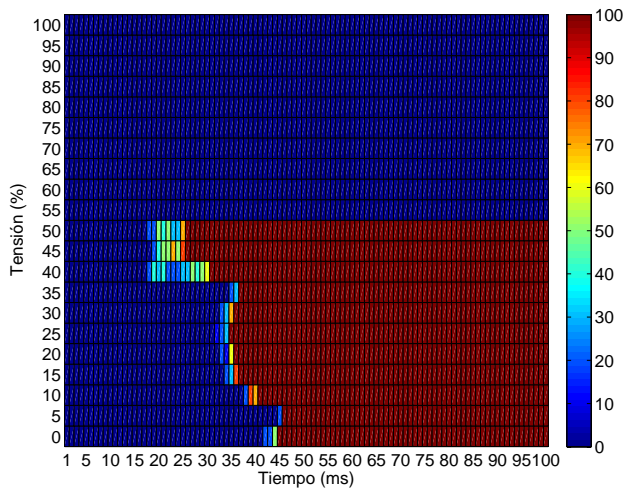

(d) Probabilidad de fallo para $45^{\circ}$.

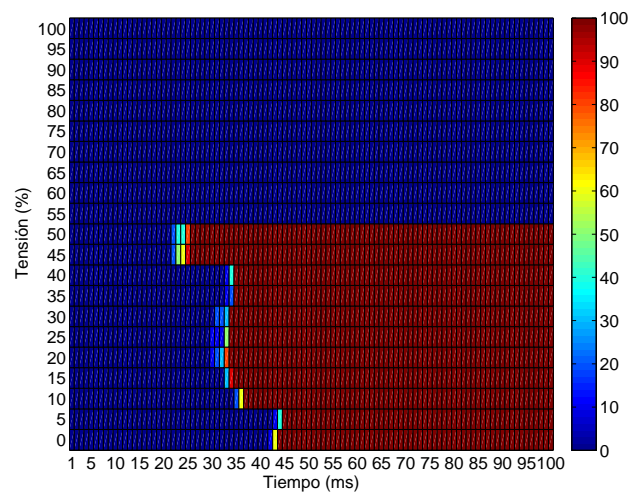

(f) Probabilidad de fallo para $75^{\circ}$

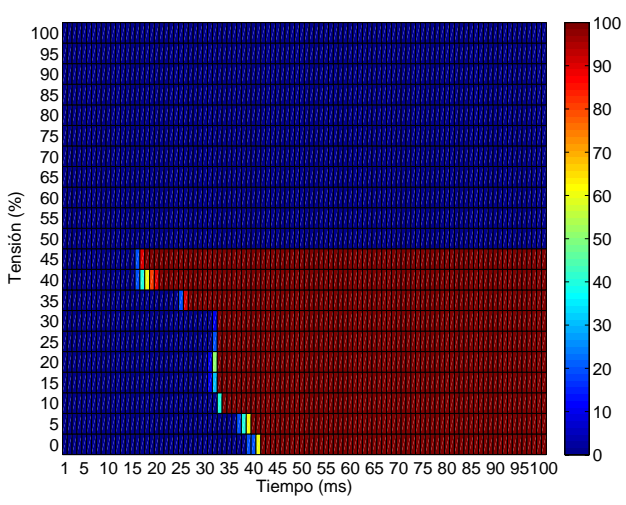

(g) Probabilidad de fallo para $90^{\circ}$.

Figura 2.27: Curvas de tolerancia del contactor Mitsubishi. Carga capacitiva. 


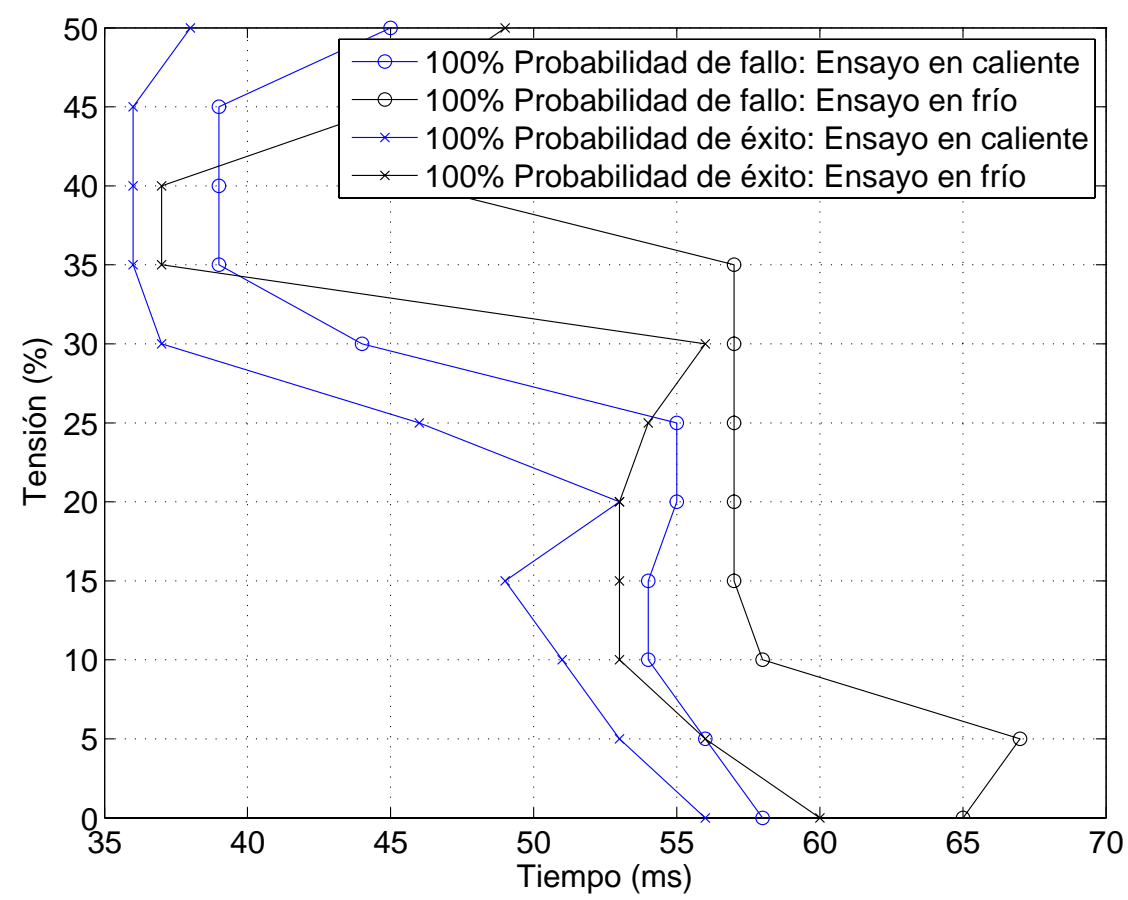

Figura 2.28: Influencia de la temperatura en la respuesta del contactor.

- en torno a $45{ }^{\circ} \mathrm{C}$ - ; mientras que el color negro representa el comportamiento cuando el contactor no ha alcanzado la temperatura de operación - la temperatura media de la bobina se mantiene en $30{ }^{\circ} \mathrm{C}$ - . Los símbolos " $x$ " indican que no se produce el disparo del equipo en ninguno de los diez ensayos realizados a una tensión residual y duración determinados; mientras que los símbolos " $O$ " indican que el contactor ha disparado en el $100 \%$ de los ensayos.

De esta manera, los dos trazos en color azul de la figura 2.28 equivalen a las condiciones de $0 \%$ y $100 \%$ de probabilidad de disparo de la curva de tolerancia mostrada previamente en la figura 2.15a

Por lo tanto, la figura 2.28 justifica que la temperatura de operación de la bobina del contactor modifica la curva de tolerancia del equipo. Concretamente, cuando la temperatura de operación es reducida, el contactor puede soportar huecos de tensión de mayor duración. Por ejemplo, para una tensión residual del $5 \%$, si la temperatura de la bobina se encuentra cercana a los $30{ }^{\circ} \mathrm{C}$, el contactor aguanta conectado hasta los $67 \mathrm{~ms}$; mientras que sólo soporta $56 \mathrm{~ms}$ si lleva conectado unas horas y ya ha alcanzado su temperatura de funcionamiento normal.

Sin embargo, también hay que tener en cuenta que en la figura 2.28 se observa que en la mayoría de los ensayos realizados en frío la zona de disper- 
sión es más extensa que en los ensayos realizados en caliente. Por lo tanto, aunque cuando la bobina se encuentra a menor temperatura se aumenta el tiempo que el contactor puede soportar huecos de tensión e interrupciones, también se amplía la zona de respuesta inestable de este tipo de equipamiento. En consecuencia, el parámetro temperatura de operación del contactor resulta crítico y podría provocar que un mismo hueco de tensión dentro de una industria o un parque eólico cause el disparo de un contactor pero no de otro, aún tratándose del mismo fabricante, debido a la diferente temperatura en que se encuentre operando cada contactor.

Como se ha podido observar en los resultados que se han obtenido sobre contactores, además de consolidar las ideas aportadas por el resto de autores presentadas en el apartado 2.2.1, se ha realizado un estudio en detalle de su comportamiento. De manera adicional, se ha investigado la respuesta de esta aparamenta en función del tipo de carga que estén alimentando mediante la realización de un estudio de probabilidad de disparo. Por último, también se complementado el análisis con la evaluación de la influencia de la temperatura de operación del equipo. Toda esta información está basada en más de 40.000 ensayos realizados sobre tres contactores.

\subsubsection{Ensayos realizados con el relé de falta a tierra}

La figura 2.29 muestra la curva de tolerancia del relé de falta a tierra ensayado en la presente Tesis Doctoral, definido en el apartado 2.3.2.2. Como se puede observar, se trata de una curva de tolerancia prácticamente rectangular, donde el equipo es capaz de operar con normalidad ante huecos de tensión de larga duración si su profundidad no excede el $55 \%$. Ante huecos de tensión profundos, se observa que la desconexión del relé se produce para duraciones superiores a $190 \mathrm{~ms}$.

Debido principalmente a la ausencia de zonas de dispersión en los rangos de tensión ensayados, el relé de falta a tierra muestra un comportamiento más simplificado que en el caso del elemento electromecánico ensayado en el apartado 2.4.1. Además, la respuesta del relé de falta a tierra no resulta alterada en función de su temperatura de operación ni por el ángulo de inicio de la onda de tensión.

Concretamente, se ha encontrado que el relé de falta a tierra ensayado está compuesto por un relé de corriente continua, de la marca TakamisawaTakamisawa Electric es una empresa filial del grupo Fujitsu-, que funciona con una tensión nominal del circuito de control de $5 V_{c c}$. Sin embargo, tal y como se observa en la figura 2.29, su comportamiento es completamente diferente al de los contactores ensayados (aunque se trate en ambos casos de elementos electromecánicos). El motivo de esta discrepancia es que la alimentación del relé de corriente continua se hace a través de una etapa rectificadora, que convierte los $230 V_{a c}$ de la red de suministro en los $5 V_{c c}$ que necesita el relé Takamisawa para funcionar. Por lo tanto, la respuesta del 


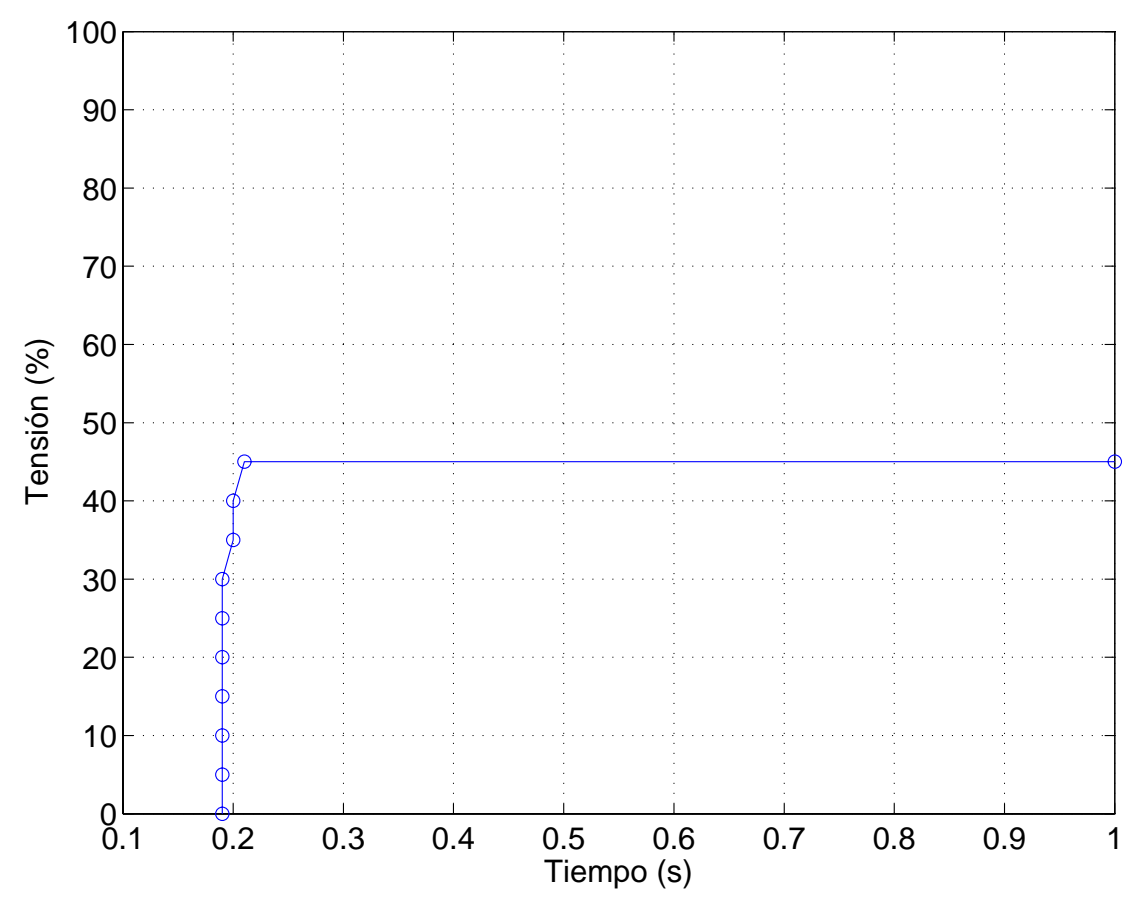

Figura 2.29: Curva de tolerancia del relé de falta a tierra.

todo el conjunto, es decir, del relé de falta a tierra, se encuentra realmente condicionada por la respuesta de su fuente de alimentación.

\subsubsection{Ensayos realizados con el variador de frecuencia}

Aunque la revisión bibliográfica presentada en el apartado 2.2.3 sintetizó el comportamiento de este tipo de equipamiento eléctrico, también se puso de manifiesto su complejidad. Además, no se han encontrado trabajos que ensayen variadores de frecuencia con entrada monofásica, como el que se ha ensayado en la presente Tesis Doctoral.

Por consiguiente, la figura 2.30 muestra la curva de tolerancia obtenida con el variador de frecuencia en función de la frecuencia de la señal de salida que alimenta al motor descrito en el apartado 2.3.2.3. Se observa que su forma es muy similar a la que se ha obtenido para el relé de falta a tierra, con una morfología predominantemente rectangular.

En función del resultado de la figura 2.30, se puede afirmar que la frecuencia de salida del variador de frecuencia es un parámetro determinante en la respuesta de este tipo de equipamiento ante huecos de tensión e interrupciones. Concretamente, la variación de la frecuencia de salida afecta de manera considerable sobre el tiempo que este equipo puede soportar ante una interrupción del suministro. Sin embargo, no se aprecia influencia sobre 


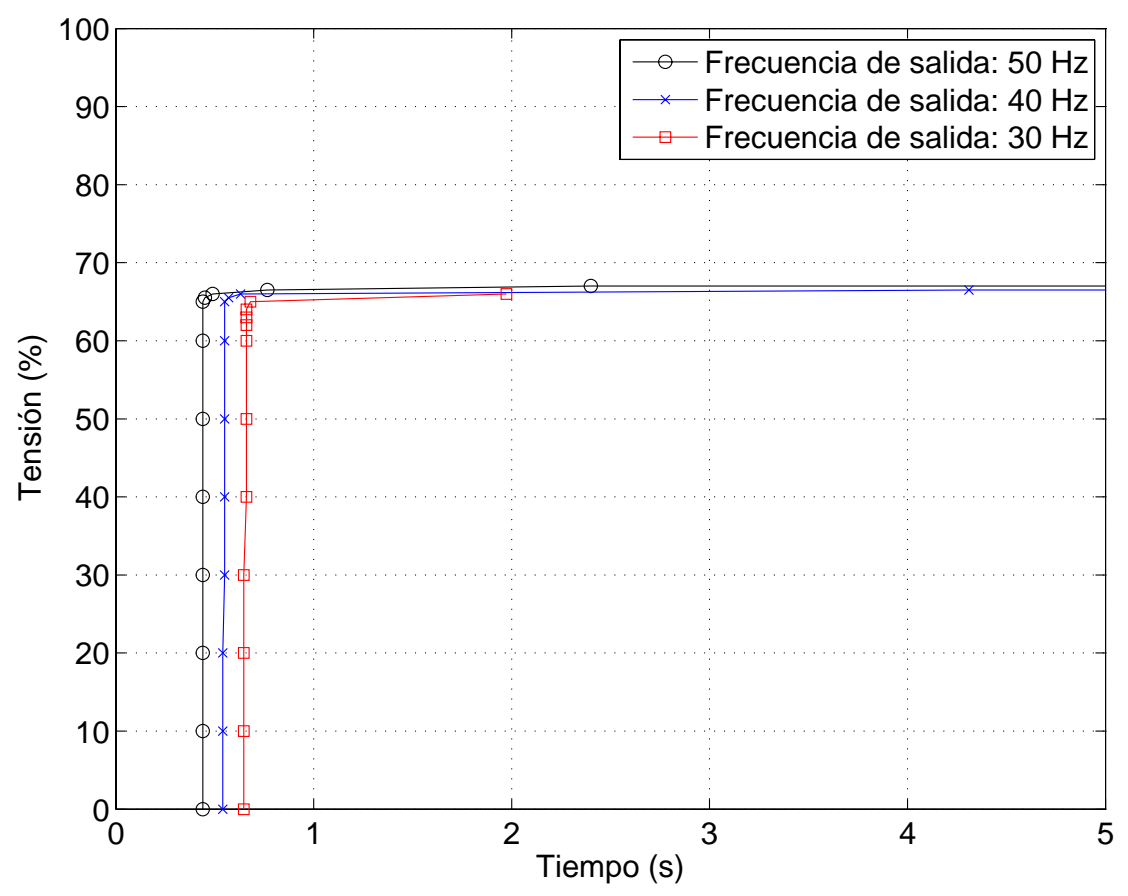

Figura 2.30: Curva de tolerancia del variador de frecuencia.

la tensión residual que puede soportar ante huecos de larga duración. Como se señaló en el apartado 2.2 .3 , este fenómeno es debido a que cuanto mayor es el régimen de carga, la intensidad que atraviesa el variador es más elevada y, por lo tanto, el límite de sobreintensidad definido por el fabricante del equipo se consigue a menores duraciones. Esta circunstancia debe ser tenida con especial consideración en aquellas instalaciones en las que se tenga motores de corriente alterna alimentados a diferente frecuencia $-\mathrm{O}$, lo que es lo mismo, a diferente velocidad de giro-; ya que un mismo hueco de tensión puede ocasionar el disparo de algunos variadores de frecuencia, pero no de otros.

Con el objeto de poder comparar la información obtenida en los ensayos con la que se ha encontrado en la revisión bibliográfica (ver tabla 2.4), la tabla 2.12 resume los valores límite registrados en los ensayos realizados en la presente Tesis Doctoral.

De esta manera, basándose en la información expuesta en la tabla 2.12 , se observa que los resultados relacionados con la tensión residual mínima, $V_{\text {res }_{\text {min }}}$, que puede tolerar el variador de frecuencia ensayado se encuentran en línea con lo experimentado por el resto de trabajos - a excepción del trabajo presentado en Vegunta et al. (2006), dado que el valor es muy diferente debido a que simula un variador de frecuencia con sistema regenerativo-. 


\begin{tabular}{c|c|c}
\hline Frecuencia de salida del variador & $V_{\text {res }_{\min }}$ & $t_{\max }$ \\
\hline $50 \mathrm{~Hz}$ & $67 \%$ & $440 \mathrm{~ms}$ \\
\hline $40 \mathrm{~Hz}$ & $67 \%$ & $540 \mathrm{~ms}$ \\
\hline $30 \mathrm{~Hz}$ & $66 \%$ & $650 \mathrm{~ms}$ \\
\hline
\end{tabular}

Tabla 2.12: Resumen de los ensayos realizados sobre huecos de tensión en variadores de frecuencia.

Por último, para finalizar el estudio del comportamiento de este tipo de equipamiento ante huecos de tensión, la figura 2.31 muestra en detalle la respuesta del variador de frecuencia ante un hueco de tensión con una profundidad del $50 \%$. En dicha figura se observa claramente cómo la intensidad de salida del variador de frecuencia (color verde) se mantiene una serie ciclos hasta que desaparece completamente. Tal y como se observa en los trazos rojo y azul oscuro (tensiones), el instante en que desaparece la intensidad entre el variador y la máquina eléctrica rotativa coincide con el momento en que la tensión de salida del variador se reduce por debajo de la de alimentación del mismo; circunstancia que se encuentra íntimamente relacionada con la descarga del condensador del bus de continua asociado al variador.

También, en vista del resultado de la figura 2.31, es importante tener en cuenta que una de las características más importantes de este tipo de equipamiento ante huecos de tensión es la variación en la velocidad del motor. De esta manera, se puede observar que, aunque el motor se continúa alimentando con la misma intensidad durante una serie de ciclos cuando el variador de frecuencia recibe el hueco, la tensión de alimentación del motor se reduce. Esta disminución de la tensión causa que el par motor se reduzca y, por lo tanto, se reduce la velocidad de giro del motor, tal y como se ha representado con el color turquesa de la figura 2.31. Evidentemente, la reducción de la velocidad de giro se enfatiza en el instante en que el variador deja de proporcionar tensión e intensidad al motor.

\subsubsection{Ensayos realizados con el ordenador personal}

La revisión bibliográfica que se presentó en el apartado 2.2.4 en relación con los $\mathrm{PC}$ señaló que el comportamiento de este tipo de cargas ante huecos de tensión e interrupciones es similar al de los variadores de frecuencia. Principalmente, esta circunstancia es debida a que, aunque un $\mathrm{PC}$ se encuentre constituido por una variada lista de componentes, el elemento principal que suministra energía a todos ellos es la fuente de alimentación del $\mathrm{PC} y$, dado que ésta posee una construcción similar al variador de frecuencia (pero sin la etapa inversora), su respuesta se puede considerar comparable. 


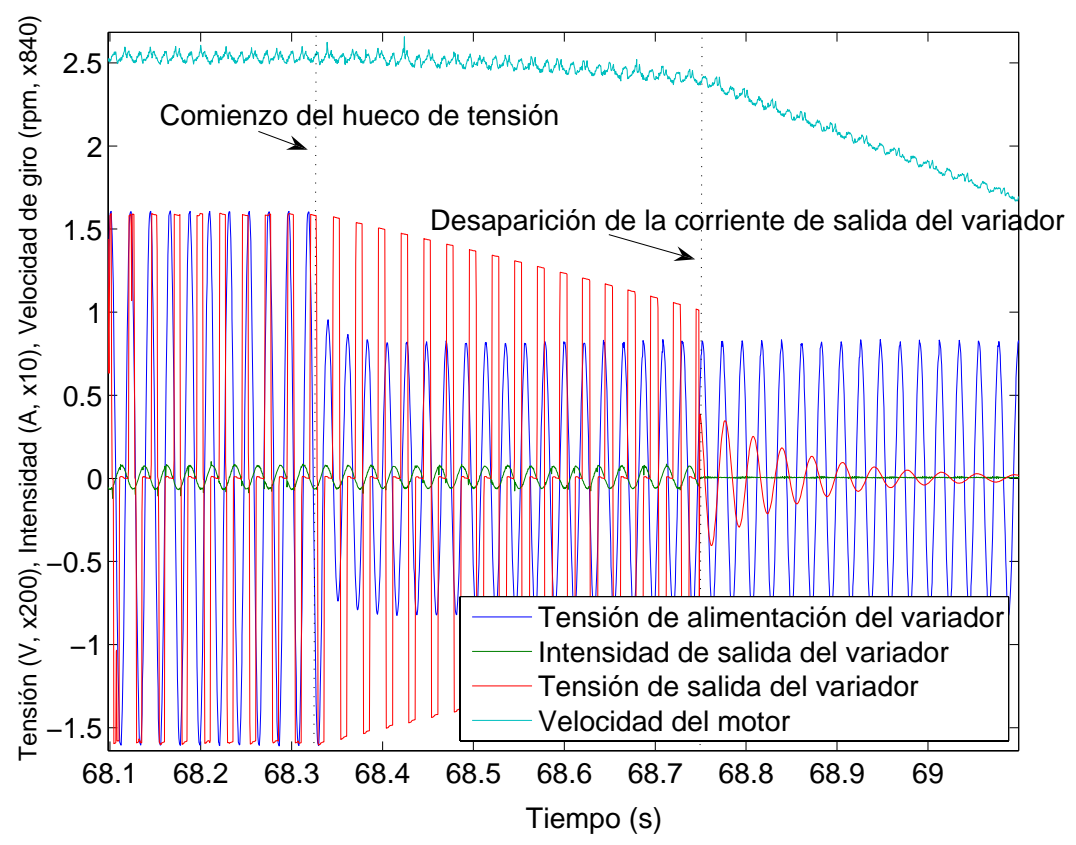

Figura 2.31: Detalle del comportamiento del variador de frecuencia.

De esta manera, la figura 2.32 muestra la curva de tolerancia obtenida mediante el ensayo del $\mathrm{PC}$ descrito en el apartado 2.3.2.4 Tal y como se comentó anteriormente, la morfología que se observa en el ensayo del PC es idéntica a la que se obtuvo con el variador de frecuencia ensayado, figura 2.30. así como con el relé de falta a tierra, figura 2.29

Observando los valores obtenidos en la curva de tolerancia presentada en la figura 2.32 se pueden sugerir algunos comentarios. Se observa que no se produce el reinicio del $\mathrm{PC}$ siempre que el hueco de tensión no tenga una profundidad superior al $45 \%$. De manera similar, el equipo puede tolerar interrupciones del suministro durante $160 \mathrm{~ms}$. Por lo tanto, el $\mathrm{PC}$ ensayado cumple holgadamente los límites impuestos por la curva ITI, así como los de la curva SEMI. También, se puede afirmar que los resultados obtenidos son coherentes con los que se mostraron en la tabla 2.5 que sintetizaba los resultados de los trabajos realizados por otros autores.

Además, los ensayos realizados sobre el $[\mathrm{PC}$ también han servido para comprobar que este tipo de equipamiento no resulta afectado por la variación del ángulo inicial del hueco, en línea con lo publicado por Saksena y Karady (2005); Djokic et al. (2005a); Hardi y Daut (2010).

Para finalizar el análisis realizado sobre $\overline{\mathrm{PC}}$, la figura 2.32 muestra la intensidad absorbida por el $\mathrm{PC}$ ensayado. En dicha figura se observa que se ha aplicado un hueco de tensión de $150 \mathrm{~ms}$ de duración que no causa el 


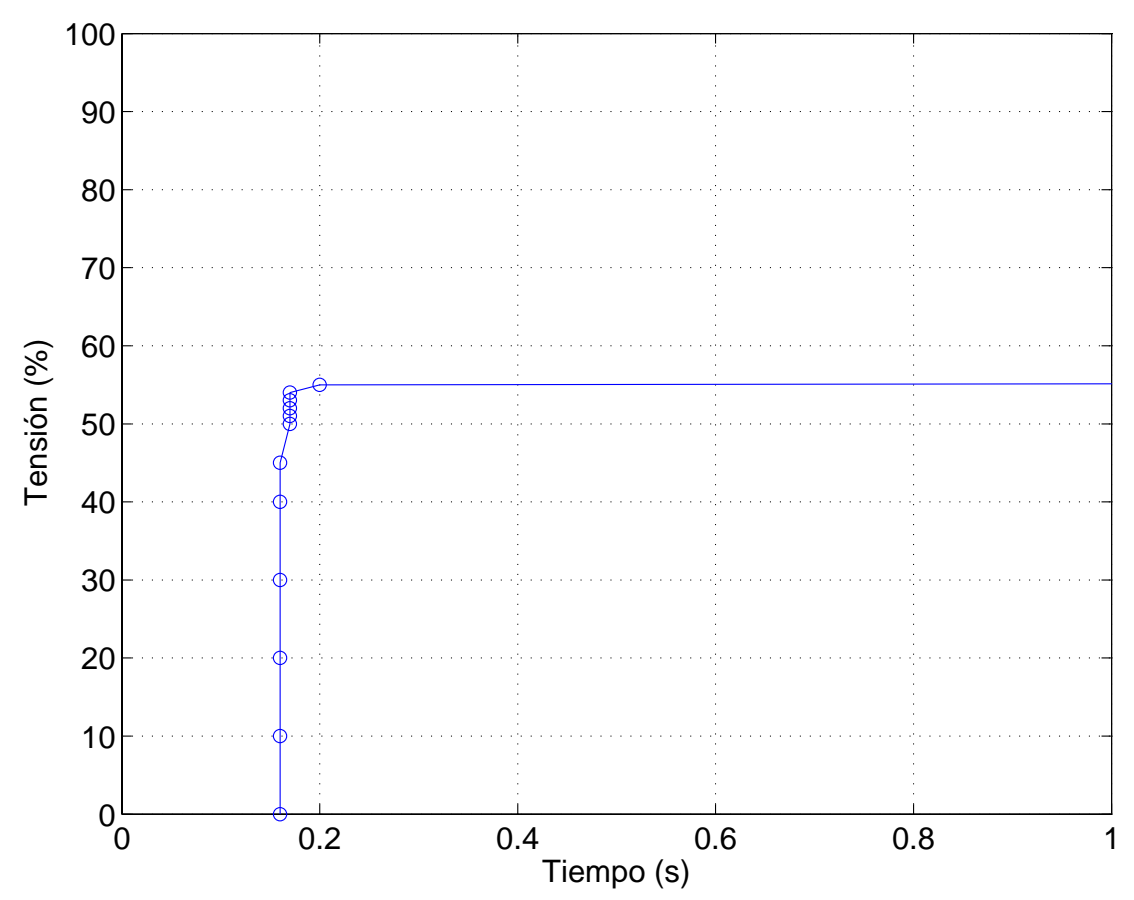

Figura 2.32: Curva de tolerancia del ordenador personal.

reinicio del equipo. Sin embargo, en color negro, se aprecia claramente la elevada intensidad que absorbe el $\mathrm{PC}$ cuando se restaura la tensión nominal. Se observa que mientras que durante el hueco de tensión el PC no absorbe intensidad de la red eléctrica, en el momento en que se restaura la tensión nominal, la intensidad absorbida por el equipo está cercana a 7 veces su valor nominal, en línea con lo publicado por Djokic et al. (2005b); Chilukuri et al. (2009); Hardi y Daut (2010). Esta elevada necesidad de intensidad es debida a la carga del condensador de la etapa de continua tras su descarga durante el hueco de tensión.

\subsubsection{Ensayos realizados con diversas cargas de iluminación}

Tal y como se señaló en el apartado 2.3.2.5, con el objeto de completar los experimentos realizados sobre diverso equipamiento eléctrico, se han realizado ensayos sobre iluminación fluorescente, inducción, y halogenuros metálicos. Es necesario señalar que actualmente estas dos últimas tecnologías no son típicamente utilizadas en el campo de la energía eólica. Sin embargo, la iluminación fluorescente representa una tipología comúnmente empleada y, dado que su respuesta se encuentra directamente relacionada con el tipo de balasto que contenga, se han ensayado también lámparas de inducción y de halogenuros metálicos para enfatizar sobre el tipo de balasto. 


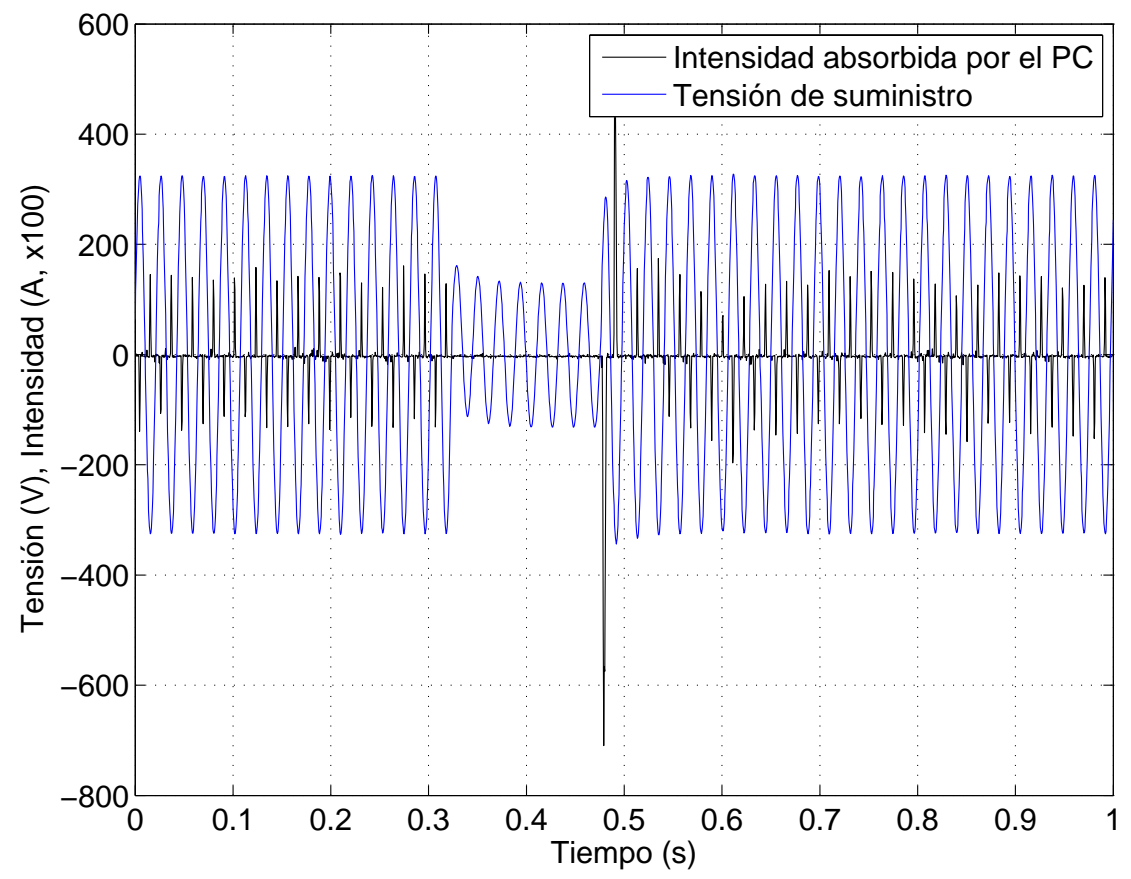

Figura 2.33: Intensidad absorbida por el ordenador personal cuando se recupera la tensión nominal. 


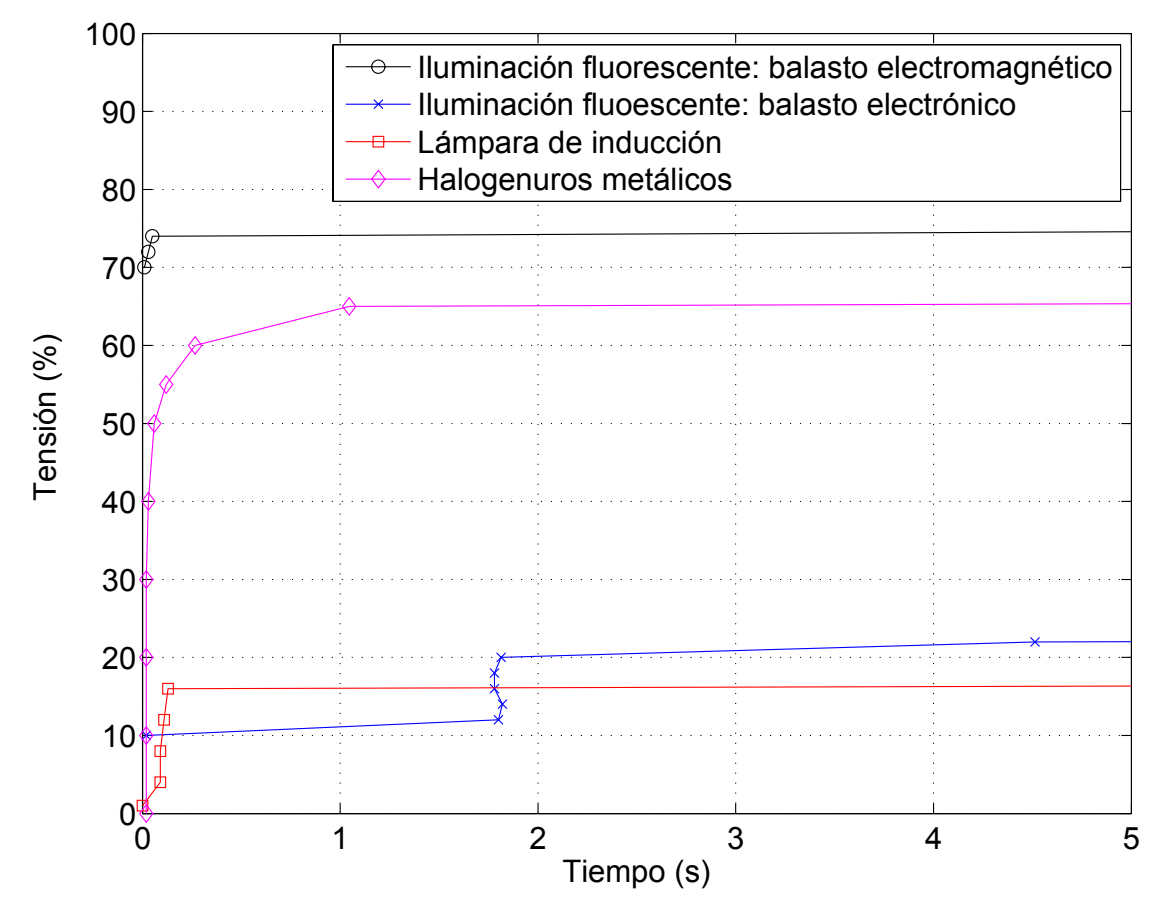

Figura 2.34: Curvas de tolerancia de diversas cargas de iluminación.

Con este motivo, la figura 2.34 muestra la curva de tolerancia obtenida para cada una de las tecnologías de iluminación que se han ensayado. La primera idea que se extrae de dicha figura es que cada tecnología se comporta de manera muy diferente ante la llegada de un hueco tensión o una interrupción. Se observa cómo el apagado de un tubo fluorescente convencional se produce para profundidades superiores al $26 \%$, mientras que el mismo tubo fluorescente alimentado a través de un balasto electrónico es capaz de soportar huecos de tensión mucho más profundos. Por lo tanto, queda justificada la mejora de la sensibilidad que existe en la iluminación fluorescente, que también había sido señalado en Drapela et al. (2009); Shareef et al. (2009b). Sin embargo, hay que recordar que la tecnología convencional tiene presencia en el $75 \%$ de las oficinas de Europa, Honrubia Escribano et al. (2012b).

Del análisis de la figura 2.34 puede extraerse que la lámpara que mejor tolera huecos de tensión de larga duración es la de inducción, dado que es capaz de mantenerse encendida mientras que la tensión residual no se sitúe por debajo del $16 \%$ de su valor nominal. Además, al igual que ocurre con la tecnología fluorescente electrónica — y, prácticamente con la convencional- el reencendido de la lámpara de inducción es instantáneo. No obstante, el elevado coste de esta tecnología causa que no se encuentre ampliamente extendida. Es posible que esta circunstancia cause que la información encontrada en la bibliografía con respecto a su comportamiento frente a perturbaciones en la 
red de suministro sea muy escasa.

Por último, en relación con la lámpara de halogenuros metálicos, en la figura 2.34 puede verse que su sensibilidad frente a huecos de tensión se sitúa en un término medio entre las dos tecnologías equipadas con balastos electrónicos y la fluorescencia convencional. Además, en los ensayos realizados, la lámpara de halogenuros metálicos ha necesitado un tiempo cercano a los 5 minutos para poder proceder a un reencendido, en línea con lo publicado en Dorr et al. (1997a); Diaz et al. (2007); Menniti et al. (2010). Por lo tanto, este tiempo de reencendido después de una desconexión imprevista debida, por ejemplo, a un hueco de tensión, representa un parámetro crítico en esta tecnología de iluminación. 


\subsection{Conclusiones}

El presente capítulo ha expuesto la temática de la calidad de suministro y analizado en profundidad la respuesta de determinado equipamiento eléctrico presente en centrales de generación de energía eólica, así como en entornos industriales, ante huecos de tensión e interrupciones del suministro.

En una primera etapa se han revisado las diferentes normativas relacionadas con esta temática. Posteriormente, mediante el extenso ensayo experimental realizado, se han obtenido las curvas de tolerancia características de diversos contactores, un relé de falta a tierra, un variador de frecuencia, un $\mathrm{PC}$ y diversas cargas de iluminación. De esta manera, además de poner de manifiesto la carencia de determinados aspectos normativos relacionados principalmente con el procedimiento de ensayo, se ha permitido conocer la respuesta esperada de este variado equipamiento cuando son sometidos a las perturbaciones más comunes que existen en las redes eléctricas.

En el caso de los contactores, más de 40.000 ensayos efectuados en la presente Tesis Doctoral posibilitan que se puedan obtener las siguientes conclusiones sobre este elemento de maniobra:

- El ángulo de inicio de la onda de tensión representa un parámetro crítico. Los ensayos en vacío de los contactores han revelado que cuando dicho ángulo se sitúa entre $0^{\circ}$ y $60^{\circ}$, este equipamiento se caracteriza de modo que a medida que aumenta la profundidad del hueco de tensión también aumenta el tiempo que el equipo puede soportar dicha perturbación. De manera inversa, mayores ángulos, hasta los $90^{\circ}$, generan una curva de tolerancia con morfología prácticamente rectangular, ampliando la máxima profundidad que puede soportar el equipo ante huecos de tensión de larga duración y reduciendo el tiempo que puede tolerar una interrupción del suministro.

- Este tipo de elemento electromecánico no posee una respuesta única ante huecos de tensión e interrupciones, sino que existe una zona de dispersión en la que la respuesta del equipo no puede ser conocida con exactitud dado su comportamiento aleatorio en dicha región. Este fenómeno se ha detectado para ángulos de inicio de la onda de tensión inferiores a $60^{\circ}$.

- Aunque los contactores posean especificaciones técnicas muy similares, los valores obtenidos en cada curva de tolerancia son diferentes en función del fabricante.

- El tipo de carga que el contactor se encuentre alimentando influye sobre la respuesta del mismo ante huecos de tensión e interrupciones. Las cargas resistivas han mostrado no tener influencia sobre su comportamiento; mientras que cargas inductivas y capacitivas modifican 
considerablemente la curva de tolerancia de este tipo de equipamiento. Concretamente, las cargas inductivas causan principalmente el aumento de la sensibilidad del equipo mediante la reducción del tiempo de disparo ante huecos de tensión de elevada profundidad, así como una reducción de la zona de dispersión. Por otro lado, la conexión de una carga capacitiva otorga al contactor una curva de tolerancia independiente del ángulo de inicio de la onda de tensión.

- La temperatura de operación del contactor también se puede considerar como un parámetro crítico. Reducidas temperaturas de operación causan que el contactor pueda soportar huecos de tensión de mayor duración. Sin embargo, las zonas de dispersión son más extensas cuando el contactor está trabajando a menor temperatura que cuando alcanza un valor constante en caliente.

Una vez que se ha realizado este extenso desarrollo experimental sobre contactores, el resto de equipamiento eléctrico ensayado ha mostrado una respuesta más sencilla ante determinadas perturbaciones de la calidad del suministro eléctrico.

En el caso del relé de falta a tierra, aunque es un elemento conectado en baja tensión, puede monitorizar intensidades de cientos de amperios, encontrándose ampliamente extendido en el sector industrial. Además, en la bibliografía consultada no se han encontrado trabajos que evalúen el comportamiento de este tipo de aparamenta eléctrica ante huecos de tensión. De esta manera, la primera impresión obtenida tras el estudio de la curva de tolerancia ensayada es su morfología rectangular. Además, la temperatura de operación no modifica dicha curva, como tampoco lo hace el ángulo de inicio de la onda de tensión. Aunque uno de los elementos principales del relé de falta a tierra es un relé de corriente continua, esta tipología de curva de tolerancia se encuentra determinada por la fuente de alimentación que suministra energía a dicho relé de corriente continua.

Por el contrario, sí que se pueden encontrar estudios relacionados con el comportamiento de variadores de frecuencia ante perturbaciones en las redes eléctricas. Por este motivo, en la presente Tesis Doctoral se ha empleado un tipo de variador muy empleado en aplicaciones de reducida potencia. Este tipo de equipamiento se emplea para controlar la velocidad de giro de motores, como puede ser el caso del sistema de control de pitch de un aerogenerador. Los ensayos realizados han permitido comprobar que, además de la forma rectangular de la curva de tolerancia, la variación de la frecuencia de salida del equipo afecta de manera considerable sobre el tiempo que puede soportar ante una interrupción del suministro. Concretamente, cuanto mayor sea la frecuencia, mayor sensibilidad posee el variador de frecuencia, dado que se reduce el tiempo que puede tolerar una interrupción. Se ha señalado que este fenómeno se encuentra íntimamente relacionado con el límite de 
sobreintensidad definido por el fabricante del equipo. También, se han efectuado mediciones sobre la velocidad de giro del motor acoplado al variador de frecuencia, indicando que este parámetro puede resultar crítico en ciertas aplicaciones.

En relación con los resultados obtenidos sobre el ordenador personal ensayado, se ha reiterado la forma rectangular de la curva de tolerancia, dado que aunque el $\mathrm{PC}$ esté compuesto de una variada lista de elementos, el componente crítico es su fuente de alimentación. Por lo tanto, puesto que la fuente de alimentación del $\mathrm{PC}$ posee una tipología de construcción similar a la de un variador de frecuencia - aunque sin incluir la etapa inversora-, su comportamiento ante huecos de tensión e interrupciones se puede considerar comparable. De una manera análoga al variador de frecuencia, el PC también presenta un elevado pico de intensidad cuando se recupera la tensión después de un hueco debido, precisamente, a la necesidad de carga del condensador de la etapa de continua.

Por último, la diversidad de tecnologías de iluminación ensayadas, algunas de ellas típicamente empleadas en parques eólicos, han permitido observar que cada una de ellas tiene un comportamiento considerablemente diferente ante huecos de tensión e interrupciones. Cuando cualquiera de las tecnologías ensayadas son conectadas a través de un balasto electrónico en lugar de electromagnético, su sensibilidad mejora de manera relevante. Por otro lado, determinadas cargas de iluminación, como la de halogenuros metálicos ensayada, presentan una característica adicional, que es el tiempo de reencendido necesario tras su desconexión; lo que en ciertas aplicaciones puede ocasionar importantes problemas.

La investigación llevada a cabo en el presente capítulo ha culminado con la publicación en uno de los congresos más relevantes a nivel internacional relacionados con las máquinas eléctricas, XIX International Conference on Electrical Machines, Honrubia Escribano et al. (2010); así como en una revista especializada que posee un elevado factor de impacto, International Journal of Electrical Power \& Energy Systems, Honrubia-Escribano et al. (2012a). 
Capítulo 3

\title{
Análisis del comportamiento de la tecnología LiDAR y evaluación de su influencia sobre la generación de energía eólica
}

\begin{abstract}
ReSUMen: El presente capítulo analiza las nuevas técnicas de medición del recuro eólico basadas en tecnología remota con el objetivo de evaluar la influencia del perfil de viento sobre la generación de energía eólica. En un primer apartado se introducen algunas nociones necesarias sobre el perfil de viento, así como de las soluciones empleadas para la medición del mismo. Se presenta también un estado del arte en este ámbito. Posteriormente se describen las características de los emplazamientos en los que se han efectuado mediciones y, finalmente, se muestran los resultados y conclusiones obtenidos. El trabajo desarrollado se enmarca dentro de las directrices seguidas por la actualización de la Norma IEC 61400-12-1.
\end{abstract}

\subsection{Introducción}

Tal y como se señaló en el apartado 1.1 de la presente Tesis Doctoral, aún con la gran evolución de la energía eólica que se ha producido en los últimos años, tanto a nivel nacional como internacional, hoy en día siguen existiendo cuestiones que no han sido tratadas con el rigor requerido. 
Actualmente, la Norma vigente relativa a la caracterización de la curva de potencia de aerogeneradores (International Electrotechnical Commission, 2005), indica que la velocidad del viento a la altura del buje del aerogenerador es el parámetro principal de entrada para la caracterización de dicha curva, junto al parámetro densidad del aire como factor secundario. Esta afirmación asume una hipótesis: la velocidad del viento es constante en toda el área barrida del rotor eólico, es decir, la velocidad del viento no varía a lo largo de todo el diámetro del rotor.

En aerogeneradores de pequeño tamaño, ya sea porque están diseñados para aplicaciones de pequeña potencia o porque sean antiguos, la suposición anterior puede ser considerada sin ningún condicionante adicional. Sin embargo, cada vez se tiende en mayor medida hacia aerogeneradores de mayor altura y diámetro de rotor que aprovechen más la energía que lleva el viento, tal y como se ha representado en la figura 1.5. Por lo tanto, la modificación de la Norma IEC 61400-12-1 es un tema de elevado interés actual dentro del sector eólico. Principalmente, la actualización de esta normativa está orientada hacia la introducción de dos conceptos, Albers et al. (2012); Honrubia Escribano et al. (2012a). Por un lado, incorporará nuevas técnicas de medida de la velocidad y dirección del viento basadas en tecnologías remotas, además de la medida sobre mástil; al igual que se ha recomendado en MEASNET (2009b). También, incluirá aspectos relacionados con el perfil de viento - también conocido como gradiente vertical de viento-, dado que ha sido comprobado por diferentes autores que éste influye de manera significativa en la generación eólica, Wagner (2010).

Además, este asunto incide de manera directa sobre la temática de la garantía de los aerogeneradores, Albers (2012). Algunos fabricantes de aerogeneradores han tenido problemas de garantía con las unidades suministradas en ciertos parques eólicos, como consecuencia de que sus aerogeneradores no han sido capaces de cumplir la curva de potencia que garantizaban debido a las condiciones particulares que existían en el emplazamiento.

La monitorización del recurso eólico a varias cotas dentro del área barrida del rotor se puede efectuar empleando diferentes soluciones tecnológicas. Lo que se viene empleando comúnmente son los sistemas de anemometría convencional instalados sobre torres meteorológicas, tratándose en la mayoría de las ocasiones de anemómetros de cazoletas, anemómetros sónicos y anemómetros de hélice. Sin embargo, dado que el coste de la torre meteorológica resultante se incrementa de manera directamente proporcional con la altura de la misma, en los últimos años se han comenzado a utilizar otro tipo de sistemas basados en la medición del recurso eólico de manera remota, Gottschall et al. (2012).

Estas modernas tecnologías remotas, en función del tipo de onda que empleen para medir el recurso eólico, se denominan LiDAR o SoDAR, y permiten la monitorización de la velocidad y dirección del viento en diferentes 
alturas definidas por el usuario sin la necesidad de emplear un mástil. No obstante, al tratarse de equipos de reciente aparición en el mercado y de mayor complejidad que los convencionales, es necesario una completa evaluación de su comportamiento.

Por lo tanto, en el presente capítulo se tratará la temática presentada en las líneas anteriores, tanto desde el punto de vista teórico como del experimental, mediante el empleo de un sistema LiDAR en diversos parques eólicos distribuidos por España.

El resultado de la investigación se espera que vea su efecto con la publicación de la Norma IEC 61400-12-1 Ed.2. La última información que se posee sobre este ámbito es que se encuentra en versión CD desde noviembre del año 2011, en la que todos los miembros pertenecientes al Comité Técnico de la misma son requeridos para alegar los comentarios oportunos y así construir la versión definitiva de la Norma, estando prevista su publicación para julio de 2013 .

\subsubsection{El viento. Nociones generales sobre el gradiente de viento}

El factor que más influencia tiene sobre la energía producida por una turbina eólica es la velocidad del viento. Debido a la relación cúbica existente entre la velocidad y la potencia, una pequeña variación de la velocidad del viento incidente se transformará en una gran variación de la potencia producida, de acuerdo a la ecuación de conversión de la energía eólica, ecuación (3.1).

$$
P_{\text {wind }}=C_{p} \cdot \frac{1}{2} \cdot \rho \cdot \pi \cdot R^{2} \cdot V_{\text {wind }}^{3}
$$

Donde $P_{\text {wind }}$ es la potencia eléctrica producida por el aerogenerador (en $\mathrm{W}), C_{p}$ es el coeficiente de potencia, $\rho$ es la densidad del aire $\left(\mathrm{en} \mathrm{kg} / \mathrm{m}^{3}\right.$ ), $R$ representa el radio del rotor (en $\mathrm{m}$ ), y $V_{\text {wind }}$ es la velocidad del viento a la altura del buje (en $\mathrm{m} / \mathrm{s}$ ).

Teniendo en cuenta (3.1), se comprende fácilmente que una variación del $10 \%$ de la velocidad del viento origina una variación de la potencia generada del $33 \%$. Por esta razón, es importante conocer algunas propiedades del elemento fundamental en la energía eólica: el viento.

El origen del viento se debe al calentamiento desigual que sufre la Tierra, Manwell et al. (2009). Debido a la diferente inclinación de los rayos solares sobre la Tierra, el sol calienta con mayor intensidad la zona del Ecuador que la de los polos. De esta manera, el aire menos denso del Ecuador asciende en la atmósfera y fluye hacia los polos, creando una falta de presión que atrae el aire frío de los polos, tal y como se ha representado en la figura 3.1. Por lo tanto, el viento se genera gracias a los gradientes de presión y, éstos, gracias a la energía solar. Finalmente, existen otros fenómenos, como el efecto 


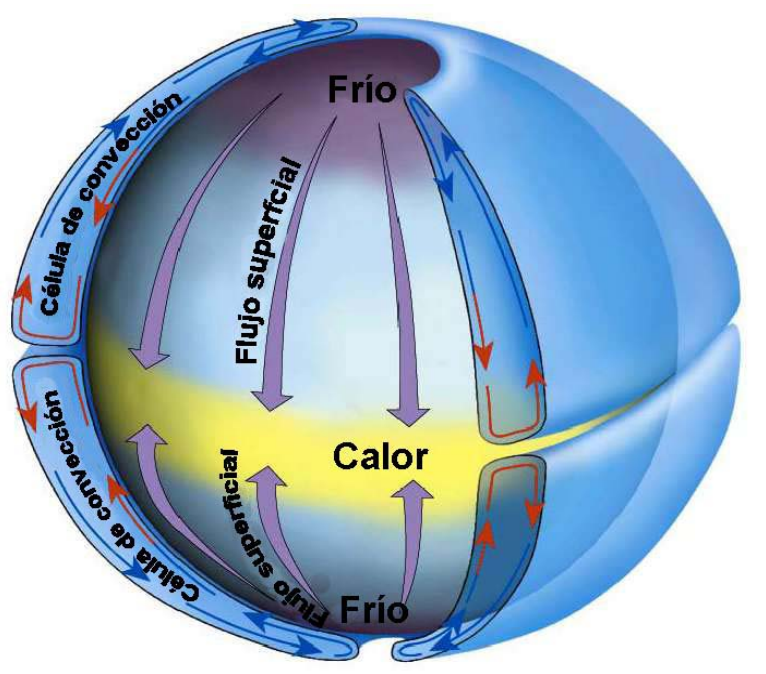

Figura 3.1: Origen del viento, Esteban Pérez (2009).

de Coriolis que, combinados con el desigual calentamiento descrito anteriormente, dan origen al viento. Concretamente, este tipo de viento se conoce como viento global o geostrófico. Finalmente, hay que tener en cuenta que existen otros fenómenos locales, como la topografía del terreno, que influyen sobre el viento global.

El viento es de naturaleza completamente estocástica, Mathew (2006). $\mathrm{Su}$ velocidad y dirección varían de manera aleatoria en función del tiempo en cualquier emplazamiento; por lo que se está en condiciones de afirmar que una de las características más importantes es su variabilidad, tanto espacial como temporal, Rodriguez Amenedo et al. (2003). Por ello, si se examinan en un lugar concreto las medidas registradas por un anemómetro, se observa que su variación es aleatoria e incluye múltiples frecuencias que van desde períodos correspondientes a $0,1 \mathrm{~s}(10 \mathrm{~Hz})$ hasta varios años.

En el pasado, los datos eólicos eran medidos y evaluados casi exclusivamente desde un punto de vista meteorológico. Sin embargo, esta información no era suficiente a la hora de abordar con cierta precisión la explotación comercial del recurso eólico. De hecho, la información meteorológica no era capaz de ofrecer información detallada sobre el aumento de la velocidad del viento con la altura o las características locales de un viento dominado por orografía compleja, por ejemplo. Según Hau (2005), desde las dos últimas décadas se vienen realizando estudios basados primordialmente en mediciones de la velocidad y dirección del viento. De esta manera, existen mapas que muestran el recurso eólico presente en la zona en base a datos reales medidos y al empleo de modelos de predicción del viento. Por ejemplo, durante el verano del año 2009, el Instituto para la Diversificación y Ahorro 


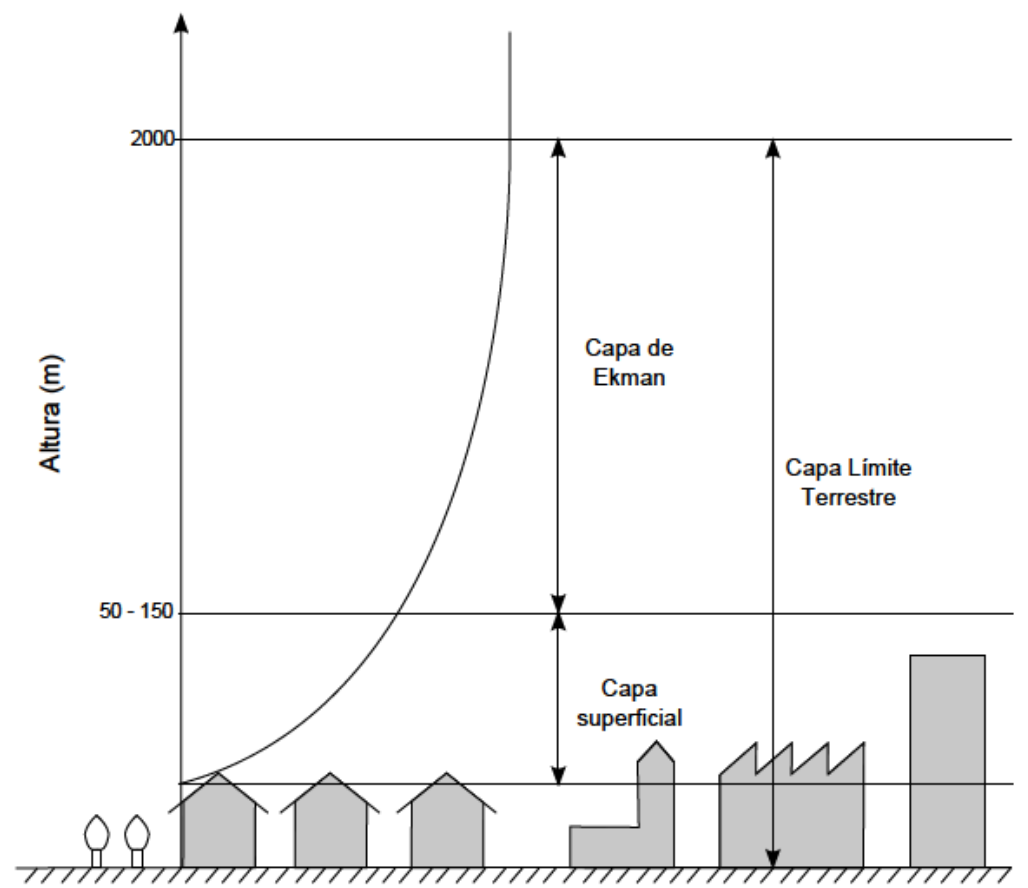

Figura 3.2: Capa límite terrestre.

de la Energía (IDAE) publicó un atlas eólico para la Península Ibérica, similar al que había desarrollado previamente el Centro Nacional de Energías Renovables (CENER), Honrubia Escribano et al. (2012a).

Una vez conocidos los vínculos entre el viento y la generación eólica, así como algunas propiedades del mismo, se van a resumir las nociones teóricas relacionadas con el gradiente de viento que resultan necesarias para la comprensión de la presente Tesis Doctoral.

La fricción de las masas de aire contra la superficie de la tierra frena la velocidad del mismo desde un valor completamente no influenciado por dicho rozamiento a elevada altitud - este viento se denominó anteriormente como viento global o geostrófico-, hasta cero directamente a nivel del suelo. En función del momento del día y de las condiciones atmosféricas, el límite de la altura en el que la superficie terrestre comienza a influir en el viento ronda los $2000 \mathrm{~m}$ sobre el nivel del suelo, aunque en noches frías y claras de poco viento puede reducirse hasta los $100 \mathrm{~m}$. Esta capa, caracterizada por la influencia de la orografía sobre el viento, se conoce como capa límite terrestre (o "atmospheric boundary layer"), representada en la figura 3.2 .

Como puede verse en la figura 3.2 la capa límite terrestre está compuesta por dos regiones, la inferior (más cercana a la superficie), y la superior o capa de Ekman. La parte inferior, conocida con el nombre de capa superficial o capa logarítmica o "Prandtl Layer" se caracteriza porque el flujo de viento 
está dominado por el rozamiento del aire con la superficie. La altura de esta capa, en función de las condiciones meteorológicas, puede situarse entre $10 \mathrm{~m}$ y $50 \mathrm{~m}$ durante las horas nocturnas, mientras que durante el día puede llegar hasta los $150 \mathrm{~m}$. Debido a estas variaciones, existen estudios realizados, Gross et al. (2002), en los que se demuestra que el buje de un aerogenerador de $60 \mathrm{~m}$ de altura se encontrará inmerso en esta capa alrededor del $32 \%$ de las horas anuales; mientras que si se trata de un aerogenerador de $100 \mathrm{~m}$ de altura de buje, éste se encontrará sólo el $7 \%$ del tiempo en dicha capa.

El aumento instantáneo de la velocidad viento con la altura depende de una serie de factores meteorológicos, como pueden ser la temperatura, y la humedad; los cuales determinan en su mayor parte la estabilidad atmosférica. La estabilidad atmosférica comúnmente se clasifica en tres estados: estable, neutra e inestable. Sin embargo, el valor medio de velocidad que será esperado a largo plazo a una determinada altura se encuentra mayoritariamente determinado por la rugosidad de la superficie terrestre, que viene definida por la rugosidad del suelo o "roughness length", $z_{0}$. En la bibliografía se encuentran numerosas tablas que definen valores numéricos de $z_{0}$, en función del tipo de terreno, $\mathrm{Hau}(2005)$. Por ejemplo, $z_{0}$ puede valer desde milímetros para superficies muy planas (hielo, agua, nieve, etc.), hasta varios metros cuando se trata de bosques o ciudades.

En la bibliografía es habitual utilizar una aproximación teórica a la variación de la velocidad del viento con la altura. Dentro de la capa superficial descrita anteriormente, la ecuación (3.2) representa una fórmula típica. Esta ecuación tiene su origen en la teoría de la capa límite de mecánica de fluidos y en investigación de la atmósfera.

$$
V_{H}=2,5 \cdot u^{*} \cdot\left|\ln \frac{H}{z_{0}}+\Psi\right|
$$

Donde, $V_{H}$ es la velocidad del viento (en $\mathrm{m} / \mathrm{s}$ ) a la altura $H$ (en m) a la que se pretende calcular, $u^{*}$ es la velocidad de fricción turbulenta (en $\mathrm{m} / \mathrm{s}$ ), y $\Psi$ es una variable que depende de la estabilidad atmosférica. El problema principal de emplear 3.2 radica en que el cálculo del parámetro $\Psi$ resulta complicado.

Debido a que $\Psi$ vale cero para atmósfera neutra, 3.2 se puede escribir utilizando una velocidad del viento de referencia, $V_{r e f}$, a una altura de referencia, $H_{r e f}$, eliminando la velocidad de fricción. De esta manera, resulta una de las ecuaciones más conocidas en el sector eólico, denominada como Ley Logarítmica, ecuación 3.3.

$$
V_{H}=V_{\text {ref }} \cdot \frac{\ln \frac{H}{z_{0}}}{\ln \frac{H_{r e f}}{z_{0}}}
$$

Es importante tener en cuenta que la validez de la expresión 3.3 está condicionada a la capa superficial de la capa límite terrestre y bajo atmós- 
fera neutra. Además, hay bastantes autores que se han dedicado a mejorar la precisión de (3.3), teniendo en cuenta, por ejemplo, la influencia de la estabilidad atmosférica, Larsen et al. (2007). Aún así, es bastante utilizada en el ámbito de la energía eólica, incluso algunos autores señalan que se ha empleado tradicionalmente en Europa desde hace muchos años, Johansson y Burnham (1992); Gipe (2004).

Otra forma alternativa de describir la variación de la velocidad del viento con la altura es la Ley Potencial, propuesta por Hellman, ecuación (3.4). Esta ecuación es más simple y posee menor fundamento teórico que la Ley Logarítmica, pero es muy empleada también en el sector de la energía eólica, Firtin et al. (2011).

$$
V_{H}=V_{\text {ref }} \cdot\left|\frac{H}{H_{\text {ref }}}\right|^{\alpha}
$$

Todas las variables mostradas en (3.4) tienen el mismo significado que en (3.3), aunque también aparece el coeficiente $\alpha$ para designar al exponente de Hellman o exponente de cortadura - también denominado como "shear exponent"- Dicho exponente es muy variable, en función de la hora del día, la estación, el tipo de terreno, la velocidad del viento y la estabilidad atmosférica. Por las noches con fuerte estabilidad puede llegar a valer 0.5 (lo que originaría un gradiente de viento muy acusado), y por el día bajar hasta 0.1 (lo que caracterizaría a un perfil de viento plano). En general, $\alpha$ aumenta con la rugosidad del terreno y disminuye con el grado de estabilidad atmosférica. Una primera imagen gráfica de lo que representa la Ley Potencial se muestra en la figura 3.3. En la parte izquierda de dicha figura se muestra un perfil de viento caracterizado por un valor de $\alpha$ elevado, originando un fuerte gradiente de viento, típico de zonas donde la rugosidad del terreno es elevada. En la zona derecha de la figura 3.3 se observa un perfil de viento "plano", debido a un valor de coeficiente de cortadura más reducido. Tal y como se observa en esta última zona, típica de lugares costeros, altas velocidades del viento se consiguen a menores alturas sobre el terreno.

El problema básico de la Ley de Potencial es la obtención del exponente de cortadura. Lo idóneo es, si se conoce la distribución del viento con la altura, calcularlo por medio de (3.4). Aunque, si lo que se quiere es obtener su valor con el objetivo de predecir la variación de la velocidad del viento con la altura, algunos autores han ajustado diferentes ecuaciones empíricas que reducen la simplicidad de la Ley Potencial, Manwell et al. (2009). Por ejemplo, Justus (1978) indica que $\alpha$ puede ser calculado a partir de una medida de la velocidad del viento a una altura conocida, a través de la expresión (3.5); mientras que Counihan (1975) aporta una solución en función de la rugosidad superficial, ecuación (3.6), similar a (3.7) aportada por Freris 

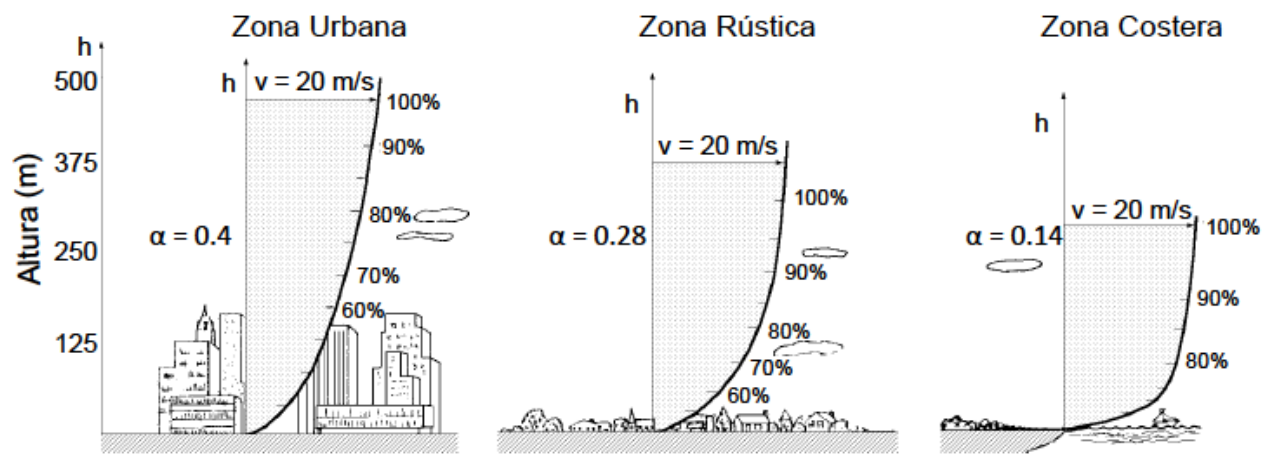

Figura 3.3: Variación de la velocidad del viento con la altura según Ley Potencial.

(1990).

$$
\begin{gathered}
\alpha=\frac{0,37-0,088 \cdot \ln \left(V_{\text {ref }}\right)}{1-0,088 \cdot \ln \left(\frac{H_{\text {ref }}}{10}\right)} \\
\alpha=0,096 \cdot \log _{10} z_{0}+0,016 \cdot\left(\log _{10} z_{0}\right)^{2}+0,24 \\
\alpha=\frac{1}{\ln \left(\frac{15,25}{z_{0}}\right)}
\end{gathered}
$$

Con el objeto de relacionar las dos Leyes más utilizadas en el sector eólico, la Logarítmica y la Potencial, en Emeis y Turk (2007) se afirma que (3.4) ofrece un ajuste casi perfecto a 3.3 bajo condiciones atmosféricas estables para ciertas rugosidades del terreno, y una buena aproximación bajo condiciones atmosféricas neutras o inestables cuando se trata de terrenos muy suaves.

A modo ilustrativo, en la figura 3.4 Honrubia et al. (2010d), se muestra una comparación de los resultados ofrecidos por ambas ecuaciones. En el eje de abscisas se representa la altura (en metros) y en el eje de ordenadas la velocidad del viento (en valores por unidad). La subfigura (a) describe la Ley Logarítmica, en función de la rugosidad del terreno, donde se aprecia que cuanta menor rugosidad posee el terreno, a bajas alturas la velocidad del viento es mayor y el perfil de viento es mucho más plano que cuando la rugosidad del terreno comienza a crecer. De manera similar, la subfigura (b) representa la Ley Potencial, en función de diferentes valores dados al coeficiente de cortadura, donde se observa que mayores valores de éste equivale a decir mayor estabilidad atmosférica - causan un elevado gradiente de viento.

El desarrollo realizado en el presente apartado ha servido para poner de manifiesto la dificultad en el cálculo del valor de la velocidad del viento a 


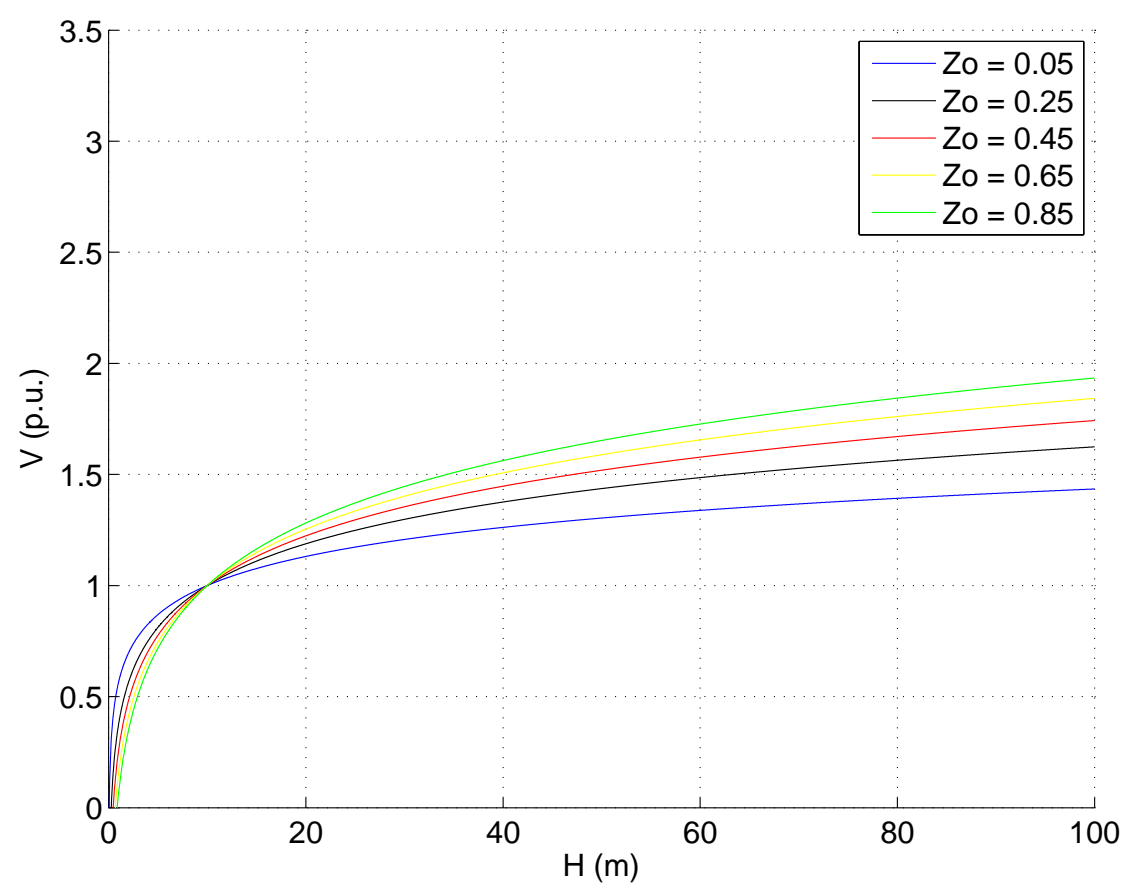

(a) Ley Logarítmica.

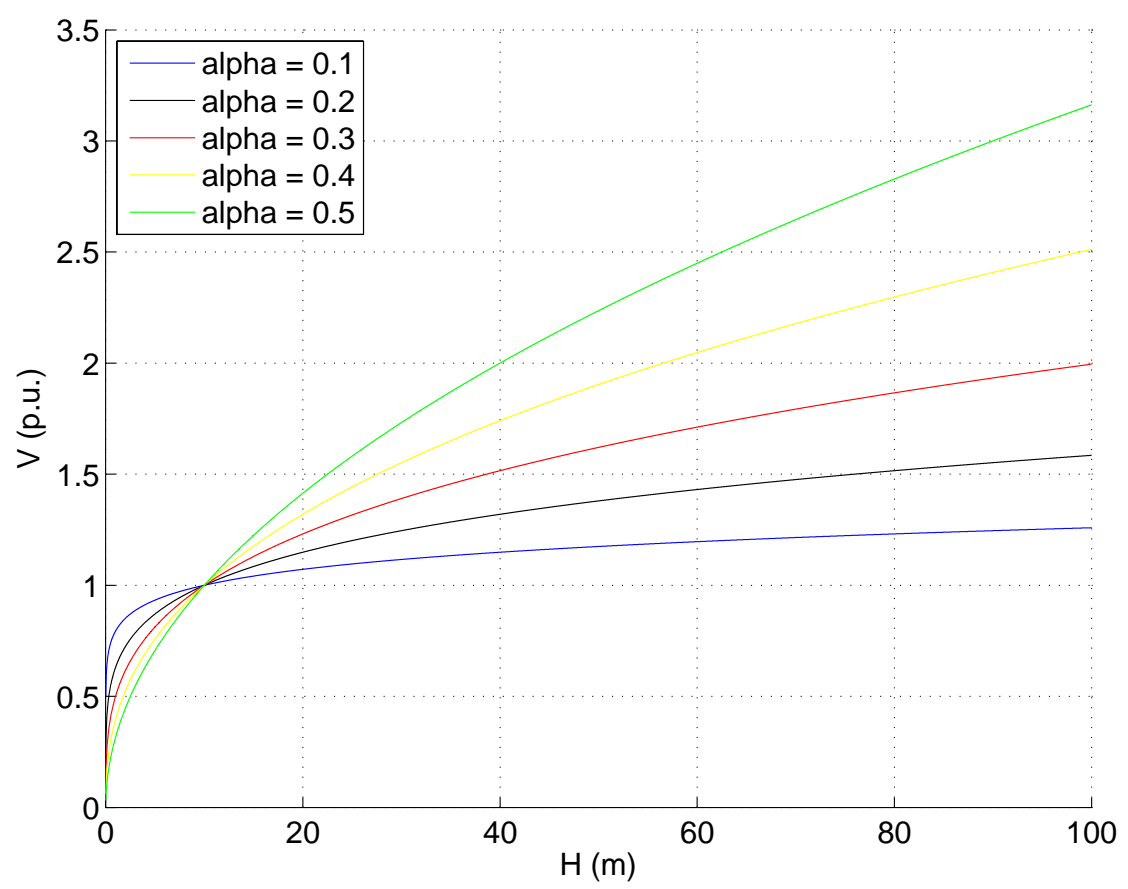

(b) Ley Potencial.

Figura 3.4: Comparativa Leyes de predicción del gradiente de viento, Honrubia et al. (2010d). 
diferentes alturas y, en mayor medida, cuando se pretende obtener un valor a elevada altura sobre el nivel del terreno. Este hecho se comprende fácilmente observando los diferentes resultados obtenidos por (3.3) y (3.4) representados en la figura 3.4 , dado que cuanto mayor es la altura a la que se quiere obtener la velocidad, mayor diferencia en el valor calculado por cada una de estas ecuaciones ampliamente extendidas en el sector eólico. Además, Hau (2005) señala que el empleo de (3.3) y (3.4) a alturas superiores a $60 \mathrm{~m}$ pierde precisión de manera significativa. En la misma línea, Firtin et al. (2011) muestra que el error en la extrapolación de velocidades mediante la utilización de estas dos ecuaciones puede generar un error del $49.6 \%$ en la energía generada por un aerogenerador de $900 \mathrm{~kW}$ de potencia nominal. Además, hay que recordar, tal y como se dedujo de la ecuación de conversión de la energía eólica (3.1), que una variación reducida de la velocidad del viento implica una variación elevada de la energía generada por el aerogenerador.

Por lo tanto, disponer de sistemas capaces de medir el recurso eólico a elevadas alturas, como mínimo a la altura del buje del aerogenerador, es una necesidad imperativa en el sector eólico. Esta exigencia se hace patente en temas relacionados con la evaluación del recurso eólico, el estudio de las cargas que se aplican sobre las palas, ensayo de curvas de potencia, cumplimiento de garantías, operación del aerogenerador, entre otras. En consecuencia, en el siguiente apartado se presentan los diferentes sistemas de medición del viento.

\subsubsection{Sistemas de medición del recurso eólico}

Además de los equipos destinados a la medición de la velocidad del viento en base a diferentes principios, existe lo que se conocen como indicadores ecológicos, basados en la forma adoptada por diversos elementos geológicos como dunas, árboles, etc., en función de la dirección y velocidad del viento en una región, Mathew (2006). Este tipo de indicadores pueden proporcionar una ligera idea de las características del viento en un lugar pero, lógicamente, la selección final de un emplazamiento como posible explotación del recurso eólico conlleva la medida real de los parámetros anteriormente mencionados. Por ello, los anemómetros colocados en largos mástiles, alcanzando actualmente más de $100 \mathrm{~m}$ de altura - la torre meteorológica más alta de Europa, implementada recientemente por el "Fraunhofer Institute for Wind Energy and Energy System Technology (IWES)", posee $200 \mathrm{~m}$ de altura-, son ampliamente utilizados.

Según el principio de funcionamiento, existen diferentes tipos de anemómetros:

- Rotación: anemómetro de cazoletas y anemómetro de hélice.

- Presión: de lámina y de tubo. 


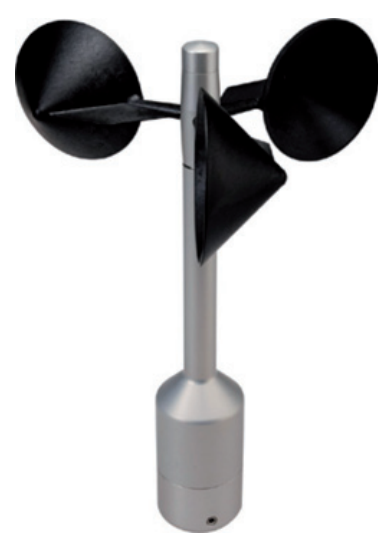

Figura 3.5: Anemómetro de cazoletas Thies First Class Adyonced.

- Termoeléctrico: anemómetro de hilo caliente y de lámina caliente.

- Efecto Doppler: anemómetro sónico, SoDAR y LiDAR.

En Honrubia Escribano et al. (2012a) se presenta un resumen de cada uno de los tipos de anemómetros nombrados anteriormente y, dado que algunas de estas tecnologías serán analizadas mediante ensayos en campo en el apartado 3.3 .3 a continuación se detallan sus propiedades.

\subsubsection{Anemómetro de cazoletas}

Este tipo de anemómetro es el más utilizado en energía eólica, y su invención se fecha en el año 1846. Básicamente está constituido por tres o cuatro cazoletas, separadas la misma distancia, unidas a un eje vertical de giro por medio de radios. La figura 3.5 muestra uno de los tipos de anemómetros de cazoletas más empleado en el sector eólico, el modelo First Class Advanced, de la compañía Thies Clima.

El principio de funcionamiento de la mayoría de este tipo de anemómetros se basa en la medida de la frecuencia de una señal, ya sea senoidal o de onda cuadrada, creada por el anemómetro. A modo de ejemplo, una función de transferencia que posee un anemómetro de cazoletas comercial es la representada en (3.8). Es decir, midiendo la frecuencia de la señal generada por el instrumento $\left(f\right.$ en $\mathrm{Hz}$ ), se obtiene la velocidad del viento $\left(V_{\text {wind }}\right.$ en $\mathrm{m} / \mathrm{s}$ ).

$$
V_{\text {wind }}=0,765 \cdot f+0,35
$$

La ventaja principal de este tipo de anemómetro es que puede trabajar en condiciones ambientales muy severas, aunque tiene ciertas limitaciones. Así, el anemómetro de cazoletas, junto con el de hélice, sobreestima la velocidad media del viento, concepto conocido como "overspeeding", Pindado 
et al. (2011). Este fenómeno es la consecuencia lógica de que el anemómetro responde más rápidamente al aumento de la velocidad del viento que a una reducción de la misma. De hecho, algunos autores han tratado de cuantificar el error cometido por estos anemómetros - definido como un error relativo resultante de restar a la velocidad medida la velocidad verdadera y dividido entre esta última- $\mathrm{y}$, aunque algunos argumentan que este error no asciende más del 3\%-5\%, otros afirman que puede llegar hasta el 10\%, Heißelmann et al. (2009). Debido a esta lenta respuesta, este tipo de anemómetros no son capaces de proporcionar mediciones precisas cuando aparecen ráfagas de viento.

En la misma línea, Sanz-Andrés et al. (2003) realiza 170 calibraciones sobre el mismo tipo de anemómetro de cazoletas, aunque suministrados por diferentes distribuidores, y se observa una seria dependencia del valor medido en función de la densidad del aire. Por lo tanto, la densidad del aire afecta a la precisión de la medida.

También, otra de las fuentes de error que pueden aparecer cuando se emplean este tipo de anemómetros montados sobre mástil es el efecto de sombra - conocido como "tower shadow"-. De esta manera, los obstáculos cercanos, así como la propia torre meteorológica que alberga al instrumento puede causar la medida errónea de la velocidad del viento. Algunos autores han señalado que la reducción de la velocidad causada por este efecto puede llegar hasta el 35\%, Stephen et al. (2011). En este sentido, las torres meteorológicas tubulares otorgan una menor reducción de la velocidad que las de celosía, Lubitz (2009).

A pesar de estas limitaciones, el anemómetro de cazoletas es uno de los más utilizados para la medida de la velocidad del viento, Heißelmann et al. (2009). Dentro de este tipo de anemómetros, además del modelo visualizado en la figura 3.5, uno de los más utilizados actualmente en la industria eólica es el modelo Maximum 40, de la compañía NRG Systems, Sanz-Andrés et al. (2003), cuyas cazoletas han registrado velocidades de hasta $96 \mathrm{~m} / \mathrm{s}$. Debido a su linealidad de salida, estos sensores son ideales para utilizar con multitud de sistemas de captación de datos y controladores.

El anemómetro de cazoletas normalmente se calibra, es decir, se obtiene la relación entre la velocidad del viento y la salida del anemómetro en un túnel de viento, con ello se obtiene una medida de la precisión del instrumento y documentación sobre su incertidumbre. De hecho, una normativa europea en este ámbito es "International Network for Harmonised and Recognised Measurements in Wind Energy (MEASNET)", la cual aplica un procedimiento de calibración del anemómetro de cazoletas en túnel de viento, MEASNET (2009a). No obstante, la calibración en túnel de viento no está exenta de errores de medida, Hansen et al. (2012), por lo que se puede encontrar una diversidad de trabajos desarrollados, Sanz-Andrés et al. (2003); Paulsen et al. (2007), basados en la calibración de anemómetros en campo. 

No obstante, la calibración en túnel de viento no está exenta de errores de medida, por lo que existen cantidad de trabajos desarrollados, [17] y [20], basados en la calibración de anemómetros en campo

2.2.2. Anemómetro de hélice (windmill)

Como su propio nombre indica, suele estar compuesto por cuatro hélices Cuando la dirección del viento incidente es paralela al eje del anemómetro, éste comenzará a girar con

una velocidad proporcional a la del viento Por este motivo,

lo ideal es añadir una veleta al sensor, Figura 4

En [17] se realizan 170 calibraciones sobre el mismo tipo de anemómetro, aunque suministrados por diferentes distribuidores, y se observa una seria dependencia del valor medido en función de la densidad del aire Por lo tanto, la densidad del aire afectará a la precisión de la medida

Una ventaja fundamental de este tipo de anemómetro es que puede trabajar en condiciones ambientales muy severas, aunque tiene ciertas limitaciones Así, el anemómetro de cazoletas, junto con el de hélice, sobreestima la velocidad media del viento, concepto conocido como overspeeding Este fenómeno es la consecuencia lógica de que el anemómetro responde más rápidamente al aumento de la velocidad del viento que a una reducción de la misma De hecho, algunos viento que a una reduccion de la misma De hecho, algunos
autores han tratado de cuantificar el erropcigh anemómetros (definido como un error relativo resultante de restar a la velocidad medida la velocidad verdadera y dividido entre esta última) y, aunque algunos argumentan que este error no asciende más del $3-5 \%$, otros afirman que puede

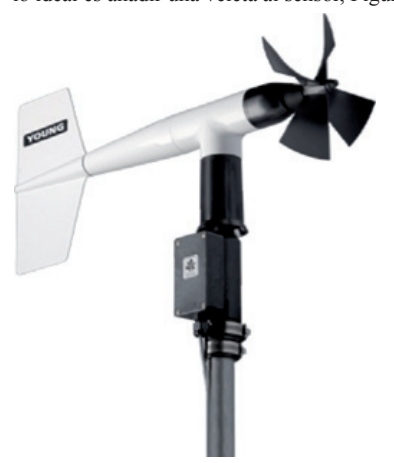

Fig. 4: Anemómetro de hélice Young Company hélice Young Compnay.

La precisión de este tipo de anemómetro puede ser similar a la del anemómetro de cazoletas, con error en torno al \pm 2\% [21] Además, como su coste también es relativamente reducido, suele utilizarse ampliamente en parques eólicos

\subsubsection{Anemómetro de hélice}

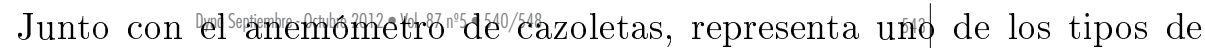
anemómetros más empleados en el sector eólico, Pindado et al. (2011). Como su propio nombre indica, suele estar compuesto por cuatro hélices, comúnmente construidas en material ligero, como aluminio o fibra de carbono.

Cuando la dirección del viento incidente es paralela al eje del anemómetro, éste comenzará a girar con una velocidad proporcional a la del viento, de manera similar a lo que ocurría con el anemómetro de cazoletas. Por este motivo, lo ideal es añadir una veleta al sensor. La figura 3.6 muestra un anemómetro de hélice de la compañía Young Company.

La precisión de este tipo de anemómetro puede ser similar a la del anemómetro de cazoletas, con error en torno al $\pm 2 \%$, Manwell et al. (2009). Además, como su coste también es relativamente reducido, suele ser ampliamente utilizado en parques eólicos.

\subsubsection{Anemómetro de lámina}

El primer tipo de anemómetro que se comenzó a utilizar fue el de lámina de presión, $\mathrm{Hau}$ (2005). Está formado por una lámina giratoria sujeta en el borde de un brazo horizontal. Este brazo está unido a un eje vertical, alrededor del cual se produce la rotación del conjunto en función de una veleta dispuesta al otro extremo del brazo.

La presión ejercida por el viento causa el giro de la lámina, de tal manera que la velocidad del viento puede ser deducida en función de la distancia recorrida durante el giro de la lámina.

\subsubsection{Anemómetro de tubo}

El otro tipo de anemómetro que utiliza la presión del viento para medir su velocidad es el de tubo, siendo el más conocido el tubo de Pitot. Se 
trata de un tubo colocado paralelamente a la dirección del viento con un orificio en su parte frontal. El aire que penetra por el orificio se remansa, y la diferencia entre la presión remanente que se genera y la presión ambiente es proporcional al cuadrado de la velocidad.

Este tipo de anemómetro puede resultar de los más precisos; aunque se utiliza principalmente para ensayos en túnel de viento en lugar de ensayos de campo, debido principalmente a que la presencia de polvo, humedad o insectos afectan a su precisión.

\subsubsection{Anemómetro de hilo caliente}

$\mathrm{Su}$ funcionamiento se basa en la pérdida de calor por convección de un hilo caliente en función de la velocidad del viento. El elemento primordial de este sensor es el hilo caliente, el cual se mantiene a una temperatura en función de la intensidad de corriente que lo atraviesa. De esta manera, midiendo el cambio de la temperatura del hilo bajo intensidad constante, o el incremento de intensidad necesario para mantener la temperatura constante, la pérdida de calor puede ser conocida y; posteriormente, aplicando teoría de convección de fluidos, se puede obtener la velocidad del viento incidente.

La principal ventaja de este anemómetro es su precisión y su reducido tiempo de respuesta pero, por el contrario, es muy frágil, necesita ser recalibrado debido a las acumulaciones de impurezas que puedan aparecer, y su coste es elevado comparado con otros tipos; por lo que su uso en la industria eólica no se encuentra ampliamente extendido.

\subsubsection{Anemómetro sónico}

Este tipo de anemómetro se basa en la relación de dependencia existente entre la velocidad de propagación del sonido y la velocidad del viento. Suele estar compuesto por tres brazos colocados en línea con otros tres. La figura 3.7 muestra un anemómetro sónico modelo WindMaster Pro, de la compañía GILL instruments.

Para su funcionamiento se hace uso de dos transductores enfrentados entre sí, constituyendo lo que se conoce como senda de medida, capaz de determinar el módulo y el sentido de la velocidad del viento en la dirección que forman los extremos de la senda. El proceso de medida se basa en la señal de ultrasonido emitida por cada transductor y que se desplaza hacia el transductor opuesto. La velocidad de propagación de ambas señales es la suma de la velocidad del sonido en el aire y de la velocidad de éste en el entorno de la senda. Por lo tanto, la velocidad del viento tiene un efecto diferente sobre la transmisión de ambas señales, modificando los tiempos de viaje con respecto a la situación en que no exista viento.

La principal ventaja de este tipo de anemómetros es que carecen de partes móviles, siendo muy precisos para velocidades de viento comprendidas entre 


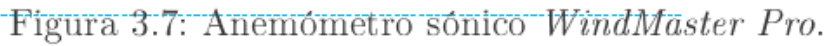

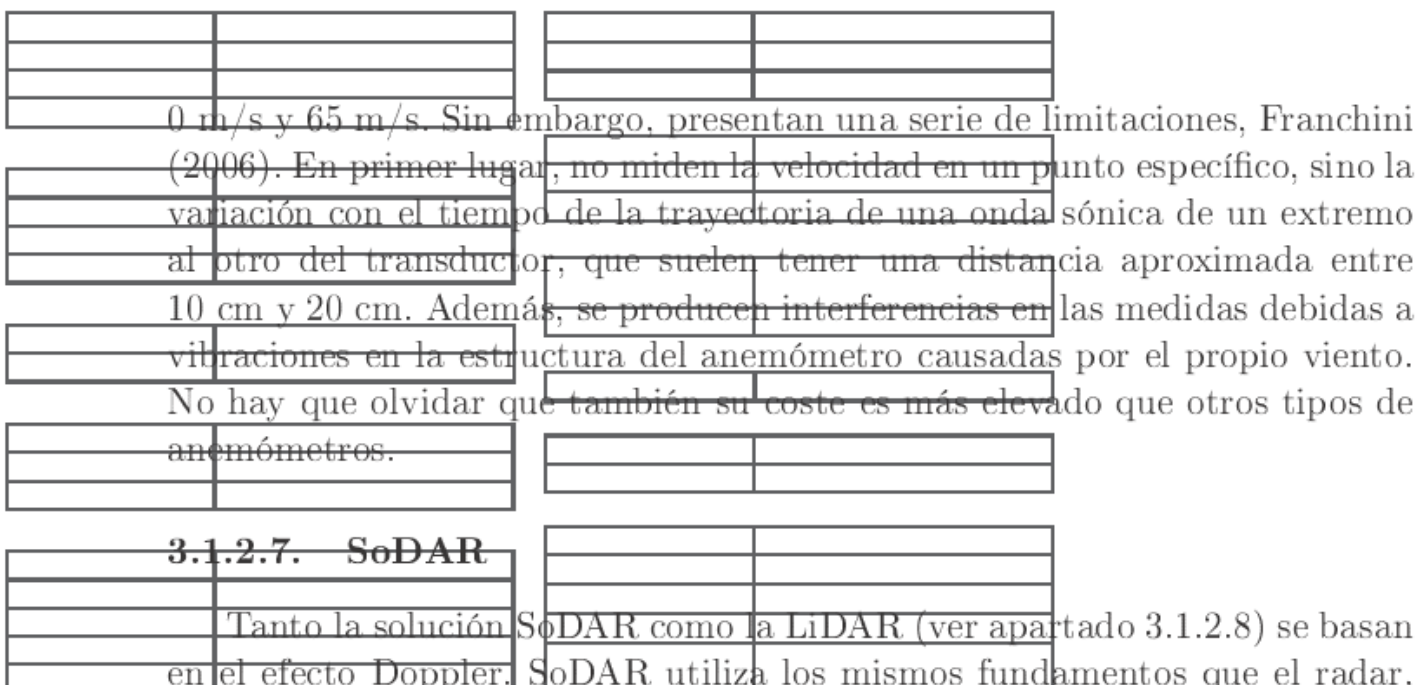
en el efecto Doppler. SoDAR utiliza los mismos fundamentos que el radar, usando ondas acústicas en lugar de microondas. Su principio de funcionamiento consiste en medir el desfase por efecto Doppler que sufre un pulso sonoro cuando éste es reflejado por una heterogeneidad del aire en movimiento. Estas heterogeneidades pueden estar provocadas por pequeñas diferencias espaciales de temperatura o de humedad, así como por el gradiente de viento. El tiempo que tarda este pulso en volver al punto desde donde fue emitido permite estimar la distancia a la que se encuentra la misma y, por lo tanto, obtener perfiles verticales de viento. Para poder calcular las distintas componentes del viento, el equipo lanza un pulso en la vertical y tres pulsos con diferentes ángulos respecto a la vertical, variando la inclinación entre $15^{\circ}$ y $30^{\circ}$. Los tiempos de llegada y las desviaciones Doppler respecto a la frecuencia original de cada uno de los tres rebotes, permiten estimar las componentes del viento en tres dimensiones.

Tal y como se ha señalado en las líneas anteriores, es importante tener en cuenta que este tipo de equipos, así como los LiDAR, tienen un principio de funcionamiento muy diferente a los anemómetros de cazoletas o de 


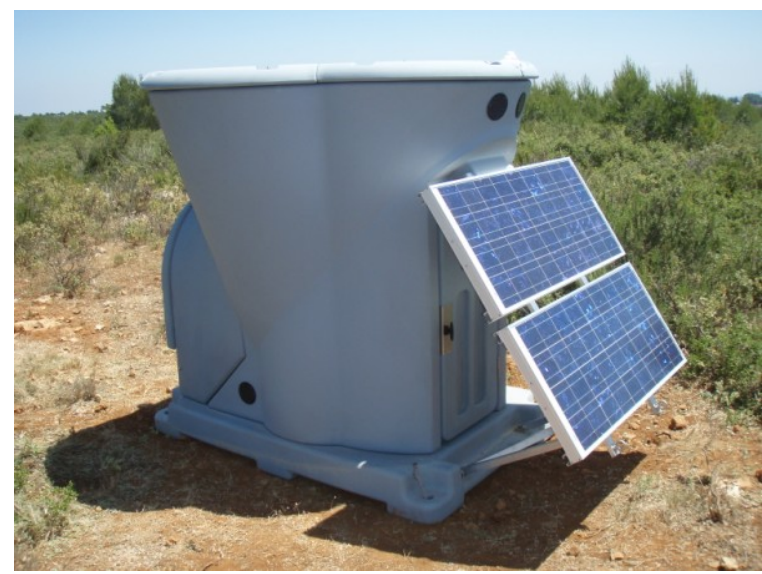

Figura 3.8: SoDAR Triton. Imagen cortesía de la empresa Windbs Techno$\log$.

hélice. Básicamente, éstos miden la velocidad del viento en un único punto - despreciando el diámetro del disco formado por las cazoletas o las hélices, respectivamente-, mientras que los equipos remotos miden las características del viento a lo largo de un volumen.

Los sistemas SoDAR han sido empleados en numerosas aplicaciones como, por ejemplo, en la predicción de dispersión de contaminantes atmosféricos, en pronósticos meteorológicos, en la predicción de la altura de nieblas, dirección de cizalladura de viento en aeropuertos, implantación de planes de emergencia en centrales nucleares, o estudios de vientos locales y terrenos complejos, Durán y Barrios (2006). Sin embargo, dentro del campo de la energía eólica y, en concreto, en la evaluación de recursos eólicos, este tipo de sistemas de anemometría no ha contado hasta hace unos pocos años con una fiabilidad suficiente como para basar en él la decisión sobre las notables inversiones requeridas por los parques eólicos; debido principalmente a que el error en la medida se situaba entre el 4-5\%, Antoniou et al. (2006). No obstante, los últimos avances en esta tecnología han permitido que desde el año 2008 el error en la medida se reduzca hasta el $2 \%$ cuando se comparan con anemómetros de cazoletas, Lang y McKeogh (2011).

Hoy en día, uno de los SoDAR con mayor implantación a nivel internacional es el modelo Triton, de la compañía SecondWind, cuya imagen en campo se muestra en la figura 3.8 .

La ventaja primordial de los equipos SoDAR es que son remotos, es decir, miden la velocidad del viento a diferentes alturas sin necesidad de construir una torre meteorológica de elevada altitud, que conllevaría un importante desembolso económico. 


\subsubsection{LiDAR}

En el año 1930, E.H. Synge fue el primero en sugerir que las medidas de densidad atmosféricas se podrían obtener analizando el retorno de haces luminosos dispersos, mediante el empleo de proyectores que iluminasen el cielo, Jaynes et al. (2007). Los primeros sistemas LiDAR, como el modelo propuesto por Synge, funcionaron en una configuración biaxial que permitía efectuar las medidas en un determinado margen. En una disposición biaxial, el detector LiDAR se sitúa a cierta distancia — hasta varios kilómetros - del punto donde la luz es transmitida a la atmósfera. El campo de visión del receptor puede ser escaneado mediante el haz del proyector, para obtener un perfil a la altura de la intensidad luminosa dispersada, mediante la aplicación de ecuaciones geométricas sencillas.

Los desarrollos posteriores en la tecnología LiDAR introdujeron la configuración monoestática, donde el transmisor y el receptor están alojados en un mismo elemento que los contiene. Esta mejora en el diseño permitió a los sistemas LiDAR incorporar transmisores con capacidad de generar una fuente pulsatoria de luz; con lo que el tiempo que tardaba en viajar el haz de luz emitido ya podía ser medido.

En el año 1938 Bureau fue el primero en utilizar un sistema monoestático pulsatorio para determinar la altura de las nubes, mostrando las técnicas de medida LiDAR de manera similar a como se conocen hoy día. La flexibilidad de las configuraciones monoestáticas permitieron, en aquel momento, conseguir medidas de perfiles verticales. El siguiente avance importante vino con la llegada del láser moderno en 1960 por Theodore H. Maiman. La capacidad para transmitir la radiación electromagnética con características específicas de frecuencia y longitud de onda ha ligado, sin lugar a dudas, el avance del LiDAR con los desarrollos de la tecnología del láser, Weitkamp (2005); Fujii y Fukuchi (2005).

Para comprender con mayor claridad el funcionamiento de un equipo LiDAR de los empleados actualmente en energía eólica, la figura 3.9 resume su modo de operación. El emisor emite una onda láser de una determinada frecuencia. Esta onda llega hasta un oscilador local que tiene la función de dividirla en dos ondas idénticas, de tal manera que una parte regresa al detector, mientras que la otra parte continúa hasta que incide sobre una partícula del aire. Al incidir sobre dicha partícula - ya sea polen, humo, polvo, etc.- , se produce su dispersión y se modifica su frecuencia. De esta manera, al detector llegan tanto la onda original como la dispersa. Después, conociendo la diferencia de las frecuencias entre la onda emitida y la dispersa, la velocidad del viento puede ser calculada mediante el efecto Doppler, Peiponen et al. (2009). Finalmente, estos equipos envían un haz láser en diferentes direcciones, en función del tipo de LiDAR, con el objetivo de obtener los parámetros del viento calculados sobre estas direcciones y así poder generar un vector resultante representativo de la velocidad y dirección del viento a 


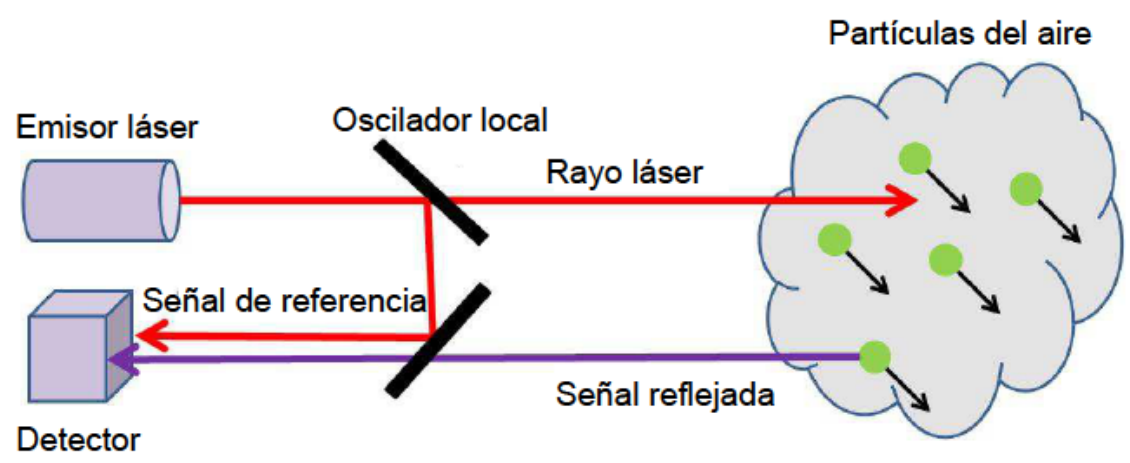

Figura 3.9: Principio de funcionamiento de un LiDAR.
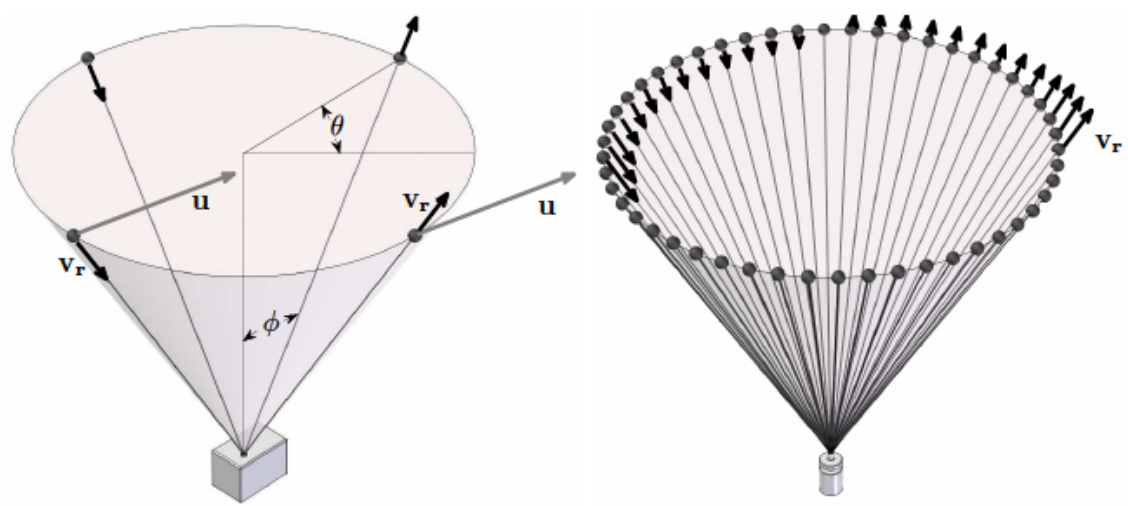

Figura 3.10: Esquema de funcionamiento de los dos sistemas LiDAR más empleados en el sector eólico, Peña (2009). A la izquierda el equipo WindCube y a la derlecha el ZephIR.

una altura determinady,, , en la figura 3.10. Peña (2009). Una idea fundamental que se deriva dié dicha figura es que fos valores $v_{r}$ obtenidos a cada altura están calculaf ós sobrê, un disco dédiáméfro variable, en función del ángulo de emisión del equipo.

Como puede deducirse de lậtpropia definición d SoDAR/y LiDAR, ambos se basan en el mismo fenómeno con 1 diferencia de que 1 primero trabaja con sonido y el segundo con luz. Debido a que la lue perıite un enfoque de mayor precisión y se dispersa por la atmósfera en menor frado que el sonido, los equipos LiDAR presentan en este sentido mejorfs propiedades que los SoDAR, Courtney et al. (2008b).

Actualmente se comercializan varios sistemas LiDAR para aplicaciones de energía eólica, aunque sólo dos se encuentran ampliamente extendidos, Braña (2011): ZephIR y WindCube, mostrados en campo en la figura 3.11 


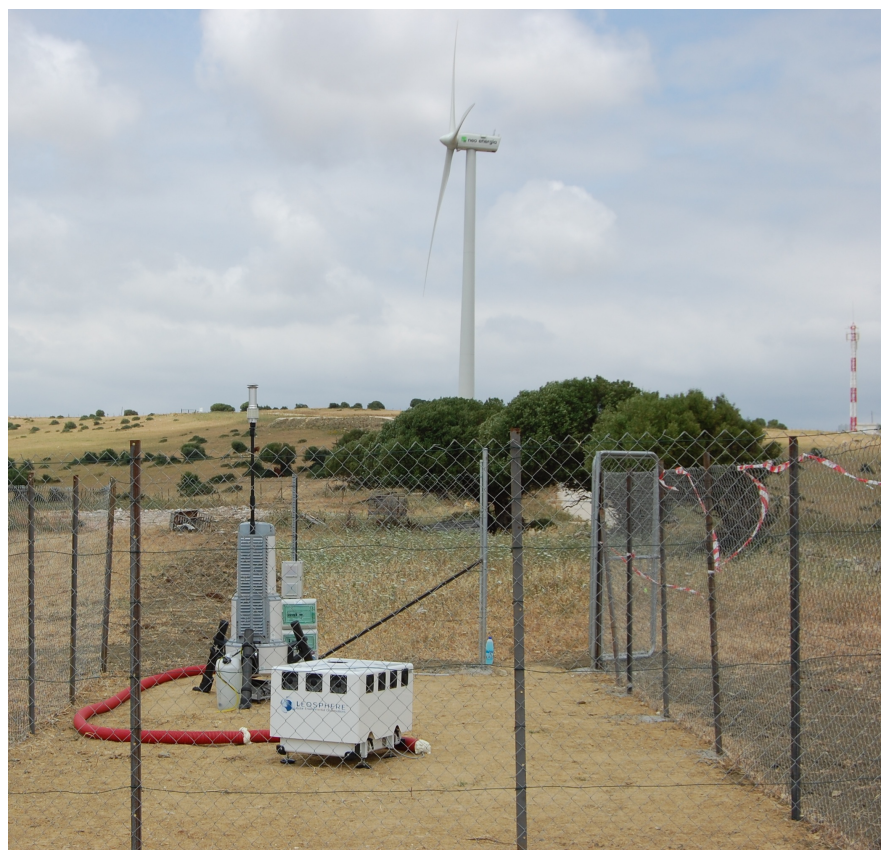

Figura 3.11: Sistemas LiDAR comerciales más empleados en el sector eólico, Honrubia et al. (2010d).

Honrubia et al. (2010d). Concretamente, el que está constituido en un único bloque es el sistema WindCube, mientras que el que se observa al fondo es el ZephIR. Aunque ambos se basan en el mismo principio de operación, mostrado en la figura 3.9, su comportamiento es sustancialmente diferente. Así pues, y dado que estas recientes soluciones se presentan como alternativas a los sistemas convencionales, a continuación se comentan sus aspectos más destacables.

El sistema ZephIR fue desarrollado por la compañía Qinetiq a principios del año 2006, aunque posteriormente la empresa Natural Power compró la licencia de explotación del mismo, Locker y Woodward (2010). Por su parte, el sistema WindCube fue introducido unos meses más tarde que ZephIR por la empresa francesa Leosphere.

Una de las diferencias más importantes entre ambos es su rango de medidas. Mientras que ZephIR es capaz de medir velocidades y dirección del viento desde una altura de $10 \mathrm{~m}$, con WindCube es imposible obtener una medición por debajo de $40 \mathrm{~m}$ de altura. Sin embargo, a cotas superiores, el WindCube es capaz de medir hasta $200 \mathrm{~m}$, limitando la altura de ZephIR en 150 m. Además, el WindCube es capaz de realizar hasta 10 medidas simultáneas en todo el rango de medida, mientras que el ZephIR sólo permite 5, y no simultáneamente, requiriendo alrededor de $3 \mathrm{~s}$ de tiempo para realizar 
The assumption is that the (mean) flow around the circumference at any height is uniform.

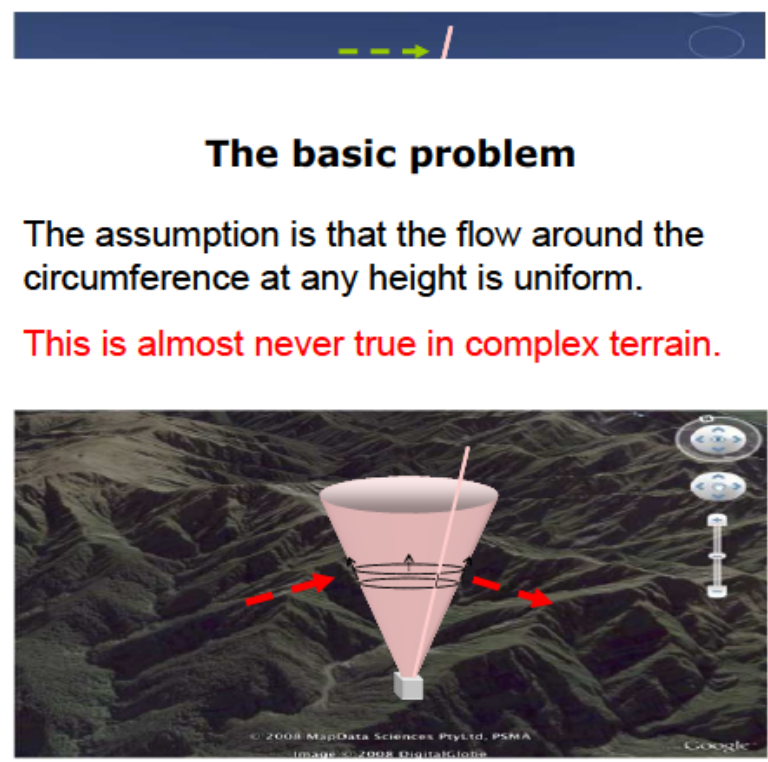

(b) Medición con sistema LiDAR bajo terreno complejo.

Figura 3.12: Consideraciones de los sistemas LiDAR bajo diferentes tipos de terrenos, Courtney 2010$)$.

cada medida (de esta manera un ciclo típico de trabajo conlleva unos $16 \mathrm{~s}$, ya que después del registro de cada altura es necesario un re-enfoque, Albers et al. (2008)). Otra diferencia a tener en cuenta es su comportamiento frente a bajas velocidades de viento; ya que el equipo ZephIR tiene problemas debidos a la introducción de errores en las medidas de velocidad del viento inferiores a $4 \mathrm{~m} / \mathrm{s}$, mientras que el WindCube puede medir velocidades más bajas sin perder precisión, Honrubia et al. (2010a).

Como característica común a ambos equipos se tiene su comportamiento en terrenos de orografía compleja, tal y como se representa en la figura 3.12 Courtney (2010). En el principio de funcionamiento de ambas soluciones se está asumiendo la hipótesis de que el flujo de viento horizontal sobre el equipo es homogéneo. Cuando se trata de un terreno llano, figura 3.12a esta suposición es realista; dado que la velocidad y dirección del viento son constantes dentro del cono láser generado por el equipo LiDAR. Sin embargo, en terreno complejo, debido a los cambios en la dirección y velocidad del viento que se pueden generar dentro del cono de emisión, puede conducir a errores en las medidas, figura $3.12 \mathrm{~b}$ dado que el vector resultante — definido como $v_{r}$ en la figura 3.10 que obtenga el equipo puede ser significativamente diferente al viento real sobre el mismo.

También es importante señalar, como punto a favor del comportamiento de estas tecnologías en terreno complejo que, en el caso del WindCube es posible sustituir el prisma de emisión convencional de $30^{\circ}$ por uno de $15^{\circ}$. 
De esta manera, se consigue reducir el error debido a flujo no homogéneo, figura 3.12b, aunque la resolución del equipo se ve notablemente afectada. De manera similar, los fabricantes de los equipos también han mejorado el algoritmo sobre terreno complejo empleando conceptos de mecánica de fluidos computacional, Parmentier et al. (2010). Aún así, el mejor método de evitar problemas sobre terreno complejo sería la instalación de tres sistemas LiDAR que enviasen el haz láser hacia el mismo punto, aunque el coste de esta solución sería demasiado elevado, Wagner (2010).

Por lo tanto, y tal y como puede deducirse de las características mencionadas en los párrafos anteriores, las mayores ventajas del empleo de este tipo de tecnologías frente a las convencionales son su portabilidad, rápida puesta en marcha, impacto ambiental prácticamente nulo, y permiten medir el recuso eólico a mayores alturas. Además, en el caso concreto del WindCu$b e$, dado que posee un disco duro interno, se pueden almacenar los datos de manera automática, con una autonomía cercana a los seis meses. También, las alturas de medida pueden ser modificadas en cualquier momento, incluso de manera remota; hecho que sería inviable sobre una torre meteorológica convencional.

Por último, es importante mencionar que los equipos comerciales mostrados en la figura 3.11 han sufrido ligeras variaciones durante estos últimos meses. La compañía Leosphere comenzó a comercializar en el año 2010 una versión más moderna del sistema WindCube, Foussekis et al. (2011), conocida como WindCube v2, con un tamaño algo inferior al que se observa en las figuras anteriores, de hecho la última versión ha reducido su peso $10 \mathrm{~kg}$. Además, dentro de su rango de medida, permite dos alturas adicionales de medición simultánea. Por su parte, el equipo explotado por Natural Power ha sufrido mayores modificaciones. La última versión, conocida como ZephIR 300, está construida en un solo bloque y tiene un consumo de energía un $30 \%$ inferior con respecto a su antecesor. Además, ha ampliado su rango de medida llegando hasta los $200 \mathrm{~m}$ de altura, comenzando a medir velocidades desde $1 \mathrm{~m} / \mathrm{s}$ y pudiendo registrar hasta 10 medidas a diferentes alturas.

Los experimentos realizados en la presente Tesis Doctoral han empleado el sistema WindCube $W L S 7$ que, técnicamente se corresponde con la versión anterior al WindCube v2, dado que la primera campaña de medidas se inició en el año 2009.

\subsubsection{Curva de potencia. Velocidad equivalente}

En este apartado se presenta en detalle cómo se realiza la caracterización de la curva de potencia de aerogeneradores, así como un reciente concepto conocido como "velocidad equivalente" introducido por Wagner (2010).

La curva de potencia de un aerogenerador consiste en representar la potencia media producida por el generador cada 10 min junto con la velocidad 
del viento media a la altura de buje durante el mismo intervalo de tiempo. Normalmente, este gráfico suele encontrarse asociado a valores dispersos que se alejan de la forma ideal de la curva. Por este motivo, en la Norma IEC 61400-12-1, se indica que se debe utilizar un método para la caracterización de la curva de potencia, conocido como método de los bin. Este método consiste básicamente en recopilar los valores medios cada 10 min de la velocidad del viento y potencia generada. Después, normalizar estos valores en función de una densidad de aire de referencia y, una vez normalizados, los datos son agrupados en conjuntos de bins de $0.5 \mathrm{~m} / \mathrm{s}$. Para que los datos tengan coherencia, la Norma indica que cada bin tiene que incluir un mínimo de $30 \mathrm{~min}$ de muestreo.

La curva de potencia obtenida con las anteriores consideraciones posee unos parámetros característicos, Rodriguez Amenedo et al. (2003). Existe una "velocidad de arranque" por debajo de la cual, el aerogenerador no produce energía. También, por encima de una determinada velocidad de viento, conocida como "velocidad nominal", el aerogenerador produce una potencia constante. Si la velocidad de viento sigue creciendo, y se sobrepasa la "velocidad de corte", entonces se produce la desconexión del aerogenerador. Desde la velocidad de arranque hasta la velocidad nominal, el aerogenerador describe la ecuación 3.1. En el apartado 3.3.4 se muestran diversas curvas de potencia registradas en un parque eólico.

Cuando se introdujo la temática de la influencia del viento en la energía eólica en el apartado 3.1.1, se señaló que el elemento más importante para la evaluación energética de un parque eólico es el viento. Lógicamente, la velocidad media del viento, $\bar{V}_{\text {wind }}$, vendrá dada por la ecuación (3.9):

$$
\bar{V}_{\text {wind }}=\frac{1}{n} \sum_{i=1}^{n} V_{i}
$$

Donde $V_{i}$ representa la velocidad registrada en el instante $i$. Sin embargo, en el ámbito de la energía eólica, utilizar la velocidad del viento media definida por (3.9) puede conducir a error. Por lo tanto, para cálculos energéticos la velocidad del viento debería ser tenida en cuenta con mayor peso en (3.9), resultando 3.10 :

$$
\bar{V}_{\text {wind }}=\left(\frac{1}{n} \sum_{i=1}^{n} V_{i}^{3}\right)^{\frac{1}{3}}
$$

Con estas dos sencillas ideas claras, investigaciones recientes en este campo de la energía eólica apuntan hacia la implementación de un nuevo concepto de velocidad del viento, conocido como "velocidad equivalente", Wagner (2010). Este concepto básicamente consiste en otorgar pesos a la velocidad registrada a varias alturas dentro del diámetro del rotor en función del área 


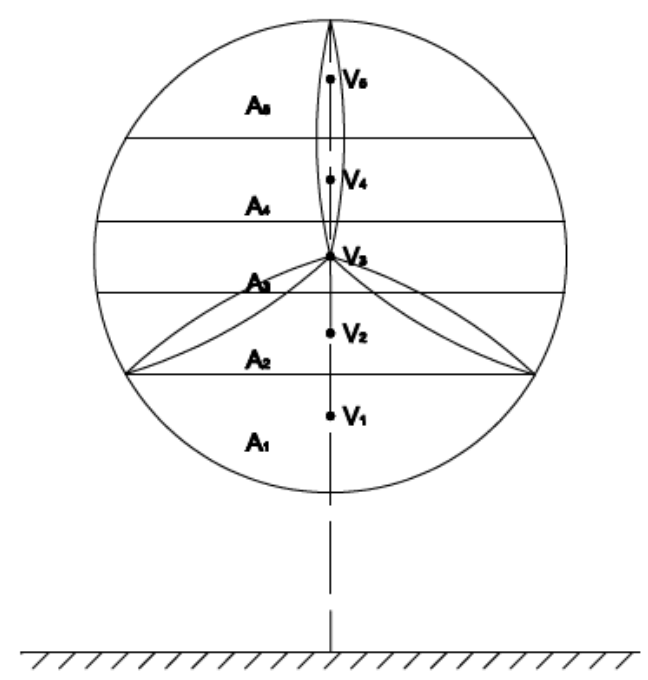

Figura 3.13: Ejemplo de partición del área barrida del rotor, Honrubia et al. (2010d).

cubierta por cada región, resultando la expresión (3.11.

$$
V_{e q 1}=\frac{1}{A} \sum_{i=1}^{n} \bar{V}_{i} A_{i}
$$

Donde $A_{i}$ representa el área del segmento circular que incluye al punto de medición de la velocidad del viento, y $A$ representa el área total barrida por el rotor. En la figura 3.13 se muestra una imagen aclaratoria, con un rotor en el que se mide la velocidad del viento en 5 alturas diferentes y, por tanto, se obtienen 5 áreas, Honrubia et al. (2010d).

Además, si se combinan (3.10) y (3.11), se puede obtener otra velocidad equivalente, ecuación 3.12 :

$$
V_{e q 2}=\left(\frac{1}{A} \sum_{i=1}^{n} \bar{V}_{i}^{3 \cdot} A_{i}\right)^{\frac{1}{3}}
$$

Se ha introducido el concepto de velocidad equivalente porque será evaluado en detalle en el apartado 3.3.4. cuando se analice su influencia sobre la generación eólica.

\subsubsection{Estado del arte}

Una vez comprendidos los principios de funcionamiento, así como las principales características de los diferentes sistemas de medición del recurso 
eólico, se presenta una revisión bibliográfica de los trabajos más relevantes que se han realizado en la temática debatida en el presente apartado.

Tal y como se señaló en el apartado 3.1, el análisis realizado en la presente Tesis Doctoral está basado en las directrices impuestas por la actualización de la Norma IEC 61400-12-1. Por lo tanto, en el apartado 3.3 se incluye, tanto la comparativa de los sistemas LiDAR con respecto a sistemas de anemometría convencional, como la evaluación de la influencia del gradiente de viento en la generación eólica. Por este motivo, en el estado del arte que se desarrolla a continuación se ha seguido el mismo orden lógico.

En Smith et al. (2006), se encuentra uno de los primeros análisis que compara la tecnología LiDAR con sistemas convencionales. Lógicamente, el sistema LiDAR empleado es un ZephIR - como curiosidad, se puede señalar que el experimento se realizó antes de la salida al mercado de dicho equipo-. El ensayo se realizó sobre terreno llano, en la "National Danish Test Station", durante un período de tres semanas. Después de solucionar un problema relacionado con la lente del equipo que les conducía a errores significativos, los resultados mostraron una gran correlación entre los datos medidos por el equipo LiDAR y los medidos por anemómetros de cazoletas en diferentes alturas. También, este primer experimento sirvió para observar que la lluvia, al igual que la nieve, inducen errores sobre la componente vertical de la velocidad del viento.

Algunos de los autores anteriores, junto a otros nuevos, realizaron en Antoniou et al. (2006) una comparativa entre dos equipos SoDAR, un LiDAR (de nuevo, el ZephIR) y la torre meteorológica, instalados en la plataforma del centro de transformación de un parque eólico offshore durante un período de dos meses. No obstante, el período efectivo del LiDAR estuvo cercano a un mes de mediciones. Sus conclusiones indican el buen resultado de ambos equipos de tecnología remota, aunque se señala que el SoDAR se desvía en mayor grado de los datos medidos por la torre debido a posibles interferencias de sonido derivadas del sistema de refrigeración del transformador.

Un año más tarde, investigadores pertenecientes al mismo centro de investigación que los anteriores junto con otros grupos de investigación de Europa vuelven a presentar comparaciones entre SoDAR, LiDAR (ZephIR) y anemómetros convencionales sobre terreno llano y complejo, Antoniou et al. (2007a). Como era de esperar, los resultados sobre terreno llano fueron altamente satisfactorios. Además, otro ensayo que realizaron fue observar la influencia la nubosidad en las medidas efectuadas con el LiDAR, señalando que, cuando aparecen nubes bajas y el sistema de filtrado que incluye el propio ZephIR no está activo, la diferencia entre las mediciones efectuadas por este equipo y por la torre meteorológica se acentúan. Por otro lado, los resultados sobre terreno complejo mostraron una peor correlación que sobre terreno llano — pendiente de ajuste con valor de 0.9177 en lugar de 0.9964 , respectivamente-. A este respecto, también hay que señalar que las medi- 
ciones que realizaron sobre terreno complejo tuvieron una duración bastante reducida, en torno a siete días efectivos de medidas. En cualquier caso, en Foussekis et al. (2007) se obtuvieron las mismas conclusiones sobre terreno complejo, incluyendo un período más largo de medidas, cercano a 16 días efectivos.

Como se ha ido viendo en los trabajos presentados anteriormente, el equipo LiDAR utilizado era el ZephIR, mientras que en Courtney et al. (2008b a) se mencionó por primera vez el sistema WindCube. De hecho, se expone una detallada comparación entre los principios de funcionamiento y márgenes de operación de cada equipo LiDAR, tal y como se ha comentado en el apartado 3.1.2.8. En esta línea, el trabajo desarrollado por Foussekis et al. (2007) se volvió a realizar en Foussekis (2009), pero en esta ocasión con los dos tipos diferentes de LiDAR más ampliamente utilizados en el sector eólico, y variando el ángulo de emisión la lente. Sus conclusiones son muy importantes, ya que afirman que la respuesta del sistema WindCube sobre terreno complejo presenta una mayor desviación estándar de la velocidad horizontal registrada cuando se utilizaba el prisma de $15^{\circ}$.

En Gottschall y Courtney (2010) se emplean simultáneamente tres equipos WindCube para comparar su respuesta con anemómetros de cazoletas sobre terreno llano, durante un período de registro de ocho semanas; aunque señalan que no pueden obtener unas firmes conclusiones debido a que el número de datos registrados no es representativo. Concretamente, sólo obtienen entre 330 muestras y 360 muestras a cada una de las alturas de registro; mientras que se recomienda que este valor no sea inferior a 600 muestras. De todas maneras, los resultados obtenidos por los tres equipos son muy similares.

Los trabajos presentados anteriormente han desembocado en la realización de diversos análisis comparativos, Honrubia et al. (2010a, 2011), que son expuestos en el apartado 3.3 .3

Continuando en la misma línea, en Cañadillas et al. (2011) se presenta uno de los estudios de mayor duración, ya que incluye un año de monitorización con un equipo WindCube en un parque eólico offshore. Se observa una reducción de la disponibilidad del equipo a medida que aumenta la altura de medición. Además, después de aplicar diversos filtros, el número de muestras queda reducido a 6.981 - hay que considerar que en un año de monitorización se deberían de haber esperado 52.560 muestras-. Con todo esto, concluyen una correlación excelente del equipo LiDAR. Debido a la importancia de la disponibilidad en el sector eólico, en Zubiaur et al. (2012) se discute en profundidad esta temática.

Recientemente, también se han publicado más trabajos relacionados con esta temática. En Foussekis et al. (2011) se comparan tanto el WindCube como el WindCube v2 con respecto a anemómetro de cazoletas y anemómetro sónico montados en mástil a $100 \mathrm{~m}$ de altura, sobre terreno complejo. 
Durante dos meses de registro (el sistema sónico sólo se usó una semana), se comparan datos en un intervalo de $8^{\circ}$ de dirección del viento, que es donde no hay ninguna influencia del mástil sobre el LiDAR ni sobre el anemómetro de cazoletas. Después de eliminar de la comparativa los datos registrados bajo lluvia, consiguen unos resultados prácticamente idénticos entre las dos versiones del equipo de la compañía Leosphere. De manera similar, en Pelletier et al. (2011) se emplea el mismo equipo en un parque eólico con unas condiciones climatológicas muy severas - temperatura mínima de $-24{ }^{\circ} \mathrm{C}$-, obteniendo un error de tan solo el $1 \%$ en relación con un anemómetro de cazoletas instalado en la torre meteorológica del parque.

En Gottschall et al. (2012), después de un filtrado exhaustivo de la monitorización realizada por un WindCube y un anemómetro de cazoletas a $116 \mathrm{~m}$ de altura, obtiene una pendiente de 0.985 y una ordenada en el origen de $0.035 \mathrm{~m} / \mathrm{s}$ sobre terreno llano. También, muestran que a mayor altura sobre el terreno, el error cometido por el equipo LiDAR es superior, dado que la pendiente de la recta de ajuste se separa más de 1 y la ordenada en el origen más de 0. Otro de los últimos estudios dentro de esta temática empleó un WindCube v2 para validarlo sobre terreno llano con respecto a anemómetro de cazoletas que se situaba sobre un mástil a una altura de $135 \mathrm{~m}$. Los resultados vuelven a ser favorables, encontrando un error entre el $1 \%$ y el $3 \%$ en la velocidad registrada por el equipo láser para velocidades del viento comprendidas entre $4 \mathrm{~m} / \mathrm{s}$ y $16 \mathrm{~m} / \mathrm{s}$, Albers et al. (2012).

Respecto a la última evolución del equipo de la compañía Natural Power, el denominado ZephIR 300, mediante calibración realizada en túnel de viento, se ha encontrado un error tan solo del $0,4 \%$ en comparación con un anemómetro de tubo de Pitot para un rango de velocidades entre $5 \mathrm{y}$ $75 \mathrm{~m} / \mathrm{s}$, Mikkelsen (2012). Estos datos apoyan los ensayos en campo realizados en Locker y Woodward (2010) con el mismo equipo.

Como se ha podido observar, todos los estudios anteriores se centran en la validación de las medidas registradas por diferentes equipos LiDAR. De esta manera, se está en condiciones de revisar los trabajos encontrados en la bibliografía relacionados con la influencia del gradiente de viento en la generación eólica.

La primera idea que hay que señalar es que, al contrario que la temática tratada en los párrafos anteriores, el análisis de los cambios de la velocidad del viento con la altura, es decir, el gradiente de viento vertical, es una cuestión tratada desde hace bastantes años en el sector eólico. En el año 1983, Sisterson et al. (1983), se presenta un estudio que emplea la Ley Potencial, ecuación (3.4), junto con medidas efectuadas por un equipo SoDAR y varios anemómetros de cazoletas, en el noroeste de Illinois. Este trabajo concluye que una ecuación teórica para describir la variación del viento con la altura, como es el caso de (3.4), podría utilizarse con cierta aproximación durante las horas del día pero, durante la noche, la ecuación se alejaba notablemente 
de los datos reales registrados. Esta situación es debida principalmente a que durante la noche los perfiles de viento eran muy irregulares, es decir, podrían darse tanto incrementos como decrementos de la velocidad con la altura, fenómeno que ha sido comentado en el apartado 3.1.1.

Bastantes años han pasado desde la publicación anterior, y todavía en la última Normativa sobre caracterización de la curva de potencia de aerogeneradores publicada en 2005, la velocidad del viento a la altura de buje es el parámetro principal para la medida de la curva de potencia del aerogenerador. Por este motivo, hoy en día son numerosos los estudios que demuestran que la curva de potencia de un aerogenerador depende un gran número de condicionantes meteorológicos y topográficos y, entre ellos, el gradiente de viento es uno de los más importantes, tal y como se ha señalado en el apartado 3.1 y se muestra a continuación.

En Langreder et al. (2004) y Bleiber et al. (2006), se centran en el impacto de la turbulencia del viento en la generación eólica. Indican que la turbulencia y el gradiente de viento están relacionados a través de la estabilidad atmosférica, dado que una alta turbulencia se corresponde con un bajo gradiente de viento, y viceversa. Además, señalan que estos dos parámetros influyen sobre la curva de potencia de aerogeneradores.

En Antoniou et al. (2007b); Wagner et al. (2009) se utilizaron dos torres meteorológicas de la "National Danish Test Station", para medir perfiles de viento hasta $160 \mathrm{~m}$ de altura durante un año. Posteriormente normalizaron los perfiles registrados y los introdujeron en un modelo eléctrico de aerogenerador. Señalaron que, midiendo la velocidad del viento en varios puntos por encima y por debajo del buje era posible la obtención de una velocidad de viento equivalente con la que calcular la energía producida por el aerogenerador, con la peculiaridad de que el gradiente de viento era tenido en cuenta. En Wagner et al. (2008) se utilizó también el concepto de velocidad equivalente, pero aplicado a mediciones efectuadas con un SoDAR y con los dos tipos de LiDAR que se encuentran actualmente ampliamente extendidos en el mercado, mostrados en la figura 3.11. De manera similar, en Wagner et al. (2010), utilizaron un equipo LiDAR para medir perfiles de viento delante de un aerogenerador a nueve alturas diferentes dentro del diámetro del rotor, en el mismo emplazamiento que los anteriores. Sus resultados muestran que la potencia generada por el aerogenerador es menor cuando los perfiles de viento no siguen una Ley Potencial.

En Antoniou y Pedersen (2009) se registró la energía generada por un aerogenerador, junto con la velocidad del viento a la altura de buje, así como a alturas inferiores al mismo en la región del Medio Oeste de los Estados Unidos. Los resultados, por un lado, muestran la existencia de un fenómeno meteorológico que genera arrastre de vientos a elevadas velocidades —entre $10 \mathrm{~m} / \mathrm{s}$ y $15 \mathrm{~m} / \mathrm{s}$ - durante la noche. Además, señalan que la extrapolación de la velocidad del viento para alturas superiores a la del buje, considerando los 
datos registrados a alturas inferiores, no representa una buena aproximación.

Por lo tanto, en base a la búsqueda bibliográfica que se ha presentado en los párrafos anteriores, queda justificado el interés del presente capítulo dentro del marco de la Tesis Doctoral. Es decir, además de la validación de un equipo LiDAR en diversos parques eólicos de España, también se incluyen aspectos relacionados con la generación de energía eléctrica de origen eólica, Honrubia et al. (2010b c d, 2012).

Por ello, en el siguiente apartado se describen los parques eólicos en los que se han llevado a cabo diversas campañas de medidas. 


\subsection{Descripción de los emplazamientos}

Un equipo de anemometría láser, concretamente un sistema WindCube, que posee el Instituto de Investigación en Energías Renovables de la Universidad de Castilla-La Mancha, ha sido empleado en la presente Tesis Doctoral para realizar mediciones de la velocidad y dirección del viento en diversos parques eólicos de España. Esta variedad de emplazamientos permite dar consistencia a las conclusiones obtenidas, dado que cada parque eólico se encuentra caracterizado por un determinado recurso eólico.

Es importante señalar que el intervalo de tiempo de registro se ha seleccionado con un valor de 10 minutos debido a que, según el espectro típico de fluctuaciones del viento, el contenido energético del viento en el período de 10 minutos y 5 horas es bastante reducido, Rodriguez Amenedo et al. (2003). Por esta razón, todos los estudios mostrados en el apartado 3.1.4 tienen en cuenta este mismo período de registro.

Asimismo, en cada uno de estos parques eólicos se cuenta con la monitorización de datos relacionados tanto, con el recurso eólico, como con la generación de energía eléctrica, con el objeto de poder evaluar la curva de potencia de los aerogeneradores.

Concretamente, se han realizado ensayos en tres parques eólicos. Dado que cada parque eólico posee unas determinadas características, se ha variado la configuración del equipo LiDAR. De esta manera, en los siguientes apartados se muestran las características de cada uno de los emplazamientos.

\subsubsection{Parque eólico 1}

El parque eólico denominado con el número "1" donde se ha instalado el equipo LiDAR se encuentra situado al sur de España. El período de registro abarca 3 meses de duración, correspondientes a la época estival del año 2009. Dicho parque eólico está compuesto por seis aerogeneradores.

Con el objetivo de obtener unos resultados coherentes, es muy importante considerar las características del terreno. Atendiendo a las recomendaciones de $\mathrm{Hau}$ (2005); Manwell et al. (2009), el terreno en los alrededores de un aerogenerador puede ser considerado llano, si:

- Las diferencias en elevación no superan 60 m en un radio de $11.5 \mathrm{~km}$.

- La relación entre el máximo desnivel y la distancia horizontal entre los dos puntos que lo provoquen, es inferior a 0.032 a lo largo de una distancia de $4 \mathrm{~km}$ en la dirección predominante del viento.

- La altura de la punta de pala inferior dentro de una distancia de $4 \mathrm{~km}$ en la dirección predominante del viento es, al menos, 3 veces superior al mayor desnivel presente dentro de los $4 \mathrm{~km}$. 


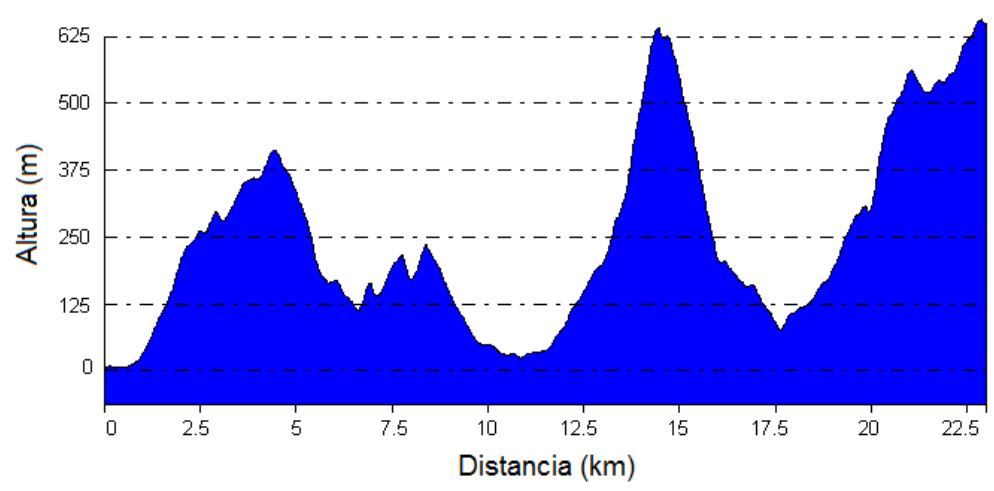

Figura 3.14: Perfil topográfico en los alrededores del parque eólico 1.

Por ello, para justificar el tipo de terreno presente en este parque eólico, en la figura 3.14 se muestra el perfil del terreno a lo largo de su dirección más crítica. Sobre esta figura puede verse que la primera de las condiciones nombradas anteriormente se encuentra muy alejada de cumplirse; por lo que se puede concluir que se trata de un terreno de orografía compleja. Especialmente, observando la figura 3.14, se puede considerar un terreno de orografía muy compleja.

En este parque eólico, el equipo WindCube se ha configurado para efectuar mediciones de la dirección y velocidad del viento en las siguientes nueve alturas: 40 m, 64 m, 67 m, 87 m, 107 m, 117 m, 127 m, 137 m, y 200 m. Asimismo, se cuenta con una torre meteorológica equipada con un anemómetro de cazoletas convencional, concretamente un Thies First Class Advanced, a $67 \mathrm{~m}$ de altura y con una veleta a $64.3 \mathrm{~m}$ de altura. La separación entre el equipo remoto y el mástil es inferior a $30 \mathrm{~m}$; por lo que se está en condiciones de efectuar un correcto análisis comparativo.

De esta manera, dado que el WindCube monitorizaba nueve alturas simultáneamente cada $10 \mathrm{~min}$, teóricamente se deberían de haber registrado un total de 13.224 muestras a cada altura. Sin embargo, debido a problemas relacionados con el suministro eléctrico del equipo, se perdieron 13 días de medida; con lo que el número definitivo de muestras por altura es de 11.298, ocasionando que la disponibilidad del equipo sea del $85 \%$.

También, tal y como se comentó en el apartado 3.1.4, debido a que su método de medida está basado en la dispersión de la luz, cuanto mayor sea la altura de medición más complicado es que realice la medición de manera correcta. Con este interés, la tabla 3.1 resume la cantidad de muestras adicionales que fueron perdidas. Sobre esta tabla se observa que en las cuatro primeras alturas el equipo pudo realizar todas las medidas pero, a partir de la quinta altura, el número de muestras perdidas aumenta de manera progresiva. 


\begin{tabular}{c|c|c|c|c|c|c|c|c}
\hline $40 \mathrm{~m}$ & $64 \mathrm{~m}$ & $67 \mathrm{~m}$ & $87 \mathrm{~m}$ & $107 \mathrm{~m}$ & $117 \mathrm{~m}$ & $127 \mathrm{~m}$ & $137 \mathrm{~m}$ & $200 \mathrm{~m}$ \\
\hline 0 & 0 & 0 & 0 & 3 & 6 & 14 & 21 & 878 \\
\hline
\end{tabular}

Tabla 3.1: Cantidad de muestras no válidas en el parque eólico 1.

Por último, dada la importancia del viento en la generación de energía eólica, también se ha realizado una breve caracterización del recurso eólico en este parque eólico, figura 3.15. La figura 3.15a muestra la distribución de velocidades a $67 \mathrm{~m}$ de altura, mientras que la figura $3.15 \mathrm{~b}$ presenta la rosa de los vientos.

A la vista de la figura 3.15, se observa que las velocidades de viento registradas han tenido un valor relativamente reducido para tratarse de un parque eólico convencional. No obstante, hay que tener en cuenta que la campaña de medidas no fue demasiado extensa y que se realizó durante los meses estivales. Por otro lado, las direcciones del viento sí que poseen una dirección sureste predominante.

\subsubsection{Parque eólico 2}

El estudio desarrollado en el parque eólico denominado como "2" ha sido realizado en un parque eólico situado al oeste de la provincia de Albacete, en la localidad de Cerroblanco, durante el período estival de 2010.

La orografía de este parque eólico también se puede considerar como compleja. Para visualizar esta circunstancia, la figura 3.16 presenta una imagen en tres dimensiones de un cuadrado de $25 \mathrm{~km}$ de lado, en donde en el centro se sitúan los equipos utilizados.

Dado que este parque eólico sólo ha sido empleado para validar las medidas del WindCube, se ha disminuido el intervalo de alturas de medición del mismo. Concretamente, las alturas de medición son las siguientes: $42 \mathrm{~m}$, $45 \mathrm{~m}, 46 \mathrm{~m}, 50 \mathrm{~m}, 55 \mathrm{~m}, 60 \mathrm{~m}, 66 \mathrm{~m}, 69 \mathrm{~m}, 70 \mathrm{~m}$, y $80 \mathrm{~m}$. Asimismo, una torre meteorológica equipada con dos tipos de anemómetros convencionales, un Thies First Class Advanced y una anemoveleta marca Young Company, modelo 05103, dispuestos ambos a $69 \mathrm{~m}$ y $45 \mathrm{~m}$ de altura. La distancia entre el equipo LiDAR y el mástil es de $50 \mathrm{~m}$, situándose el WindCube al noroeste de la torre meteorológica. De esta manera se está en condiciones de realizar un profundo análisis comparativo a dos diferentes cotas sobre el terreno.

Además, sobre este emplazamiento, con el objeto de profundizar en relación con la fiabilidad del equipo láser sobre terreno de orografía compleja, se han realizado dos series de mediciones:

- Primer período de registro: abarca una duración de 21 días durante el mes de junio de 2010, empleando el prisma de emisión convencional de 


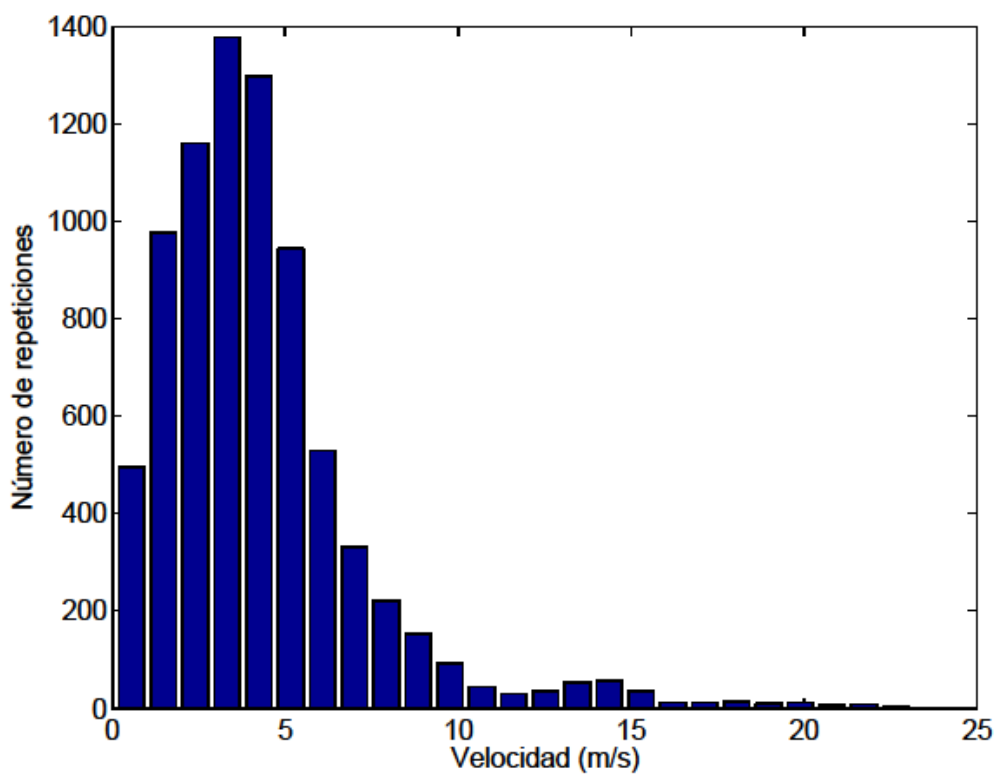

(a) Histograma de velocidades del viento.

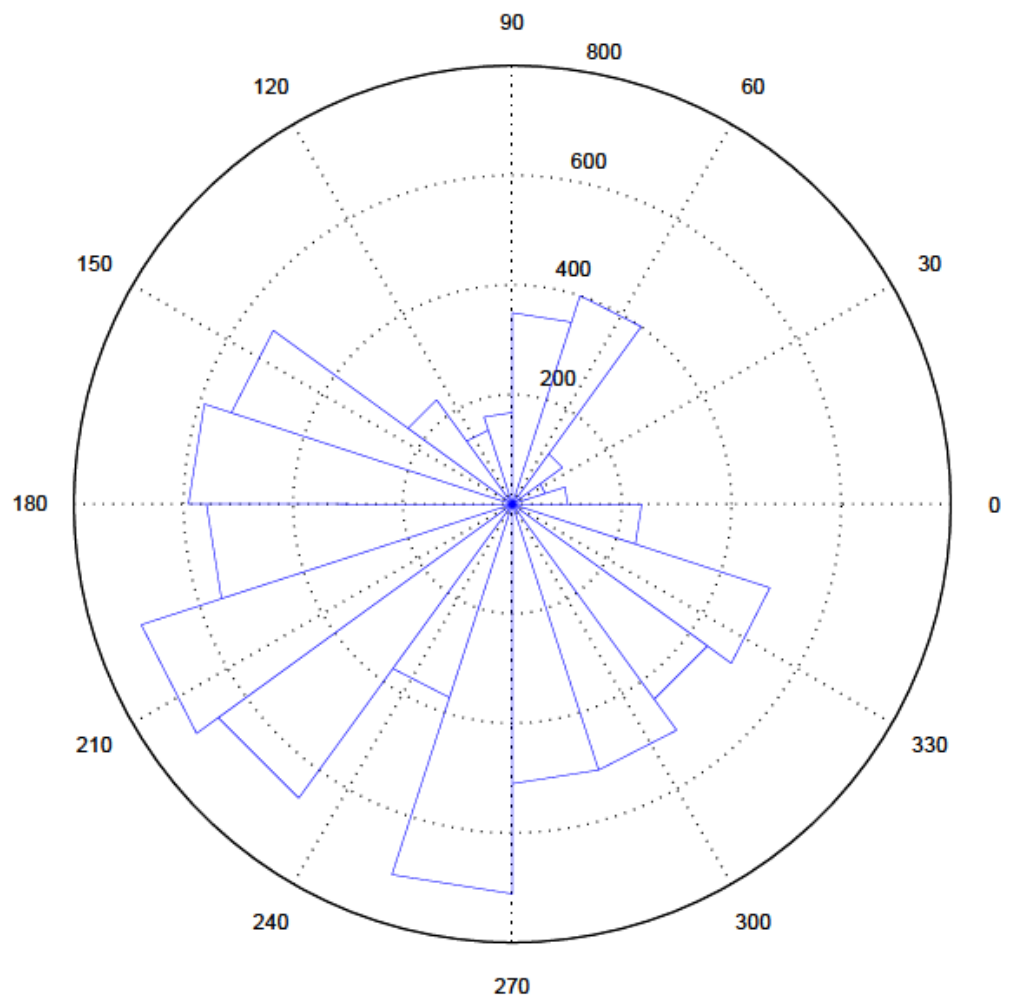

(b) Rosa de los vientos.

Figura 3.15: Características del recurso eólico en el parque eólico 1. 


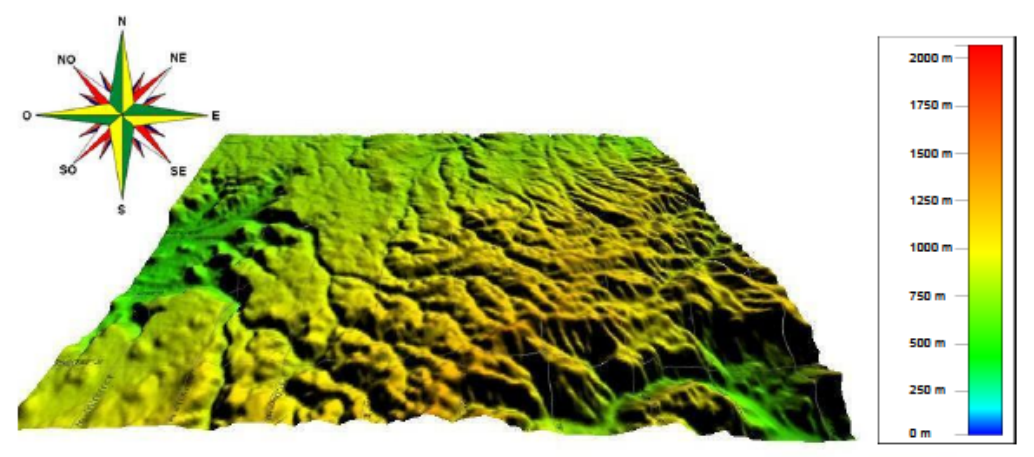

Figura 3.16: Imagen en 3D del emplazamiento 2.

$30^{\circ}$.

- Segundo período de registro: empleando el prisma de $15^{\circ}$, comprende una duración de 31 días (julio del año 2010).

Con respecto a la disponibilidad del equipo, en esta ocasión ha sido bastante variable. Durante el primer período de registro, se deberían haber registrado 3.011 muestras pero, debido a desconexiones del equipo, se perdieron 65 medidas, quedando un total de 2.946 muestras útiles. De esta manera, la disponibilidad del equipo durante estos 21 días está próxima al $98 \%$. Durante el segundo período, se redujo considerablemente la disponibilidad debido a problemas en la puesta en marcha del equipo; ya que, aunque deberían de haberse realizado 4.399 registros, sólo se pudieron conseguir 3.770 muestras, reduciendo la disponibilidad al $86 \%$.

Por último, la figura 3.17 muestra los parámetros más importantes del recurso eólico en la zona estudiada, como son la distribución de velocidades a 69 metros sobre el nivel del suelo medida por el anemómetro Thies, figura 3.17a y la sectorización de las direcciones del viento, medida por la veleta Young Company a la misma altura, figura $3.17 \mathrm{~b}$ ambos durante los meses de junio y julio de 2010 .

A la vista de la figura 3.17a se observa que las velocidades de viento registradas tienen un valor aceptable para un emplazamiento eólico, muy diferente del parque eólico 1, figura 3.15a Además, hay que tener en cuenta que se trata del período estival, donde las velocidades registradas suelen ser inferiores a las que se registran durante el invierno. Con el mismo interés, en la figura $3.17 \mathrm{~b}$ se observa una dirección suroeste predominante para el viento incidente. De todas maneras, debido al reducido tiempo de la campaña de medidas, se trata de una caracterización del recurso eólico meramente orientativa. 


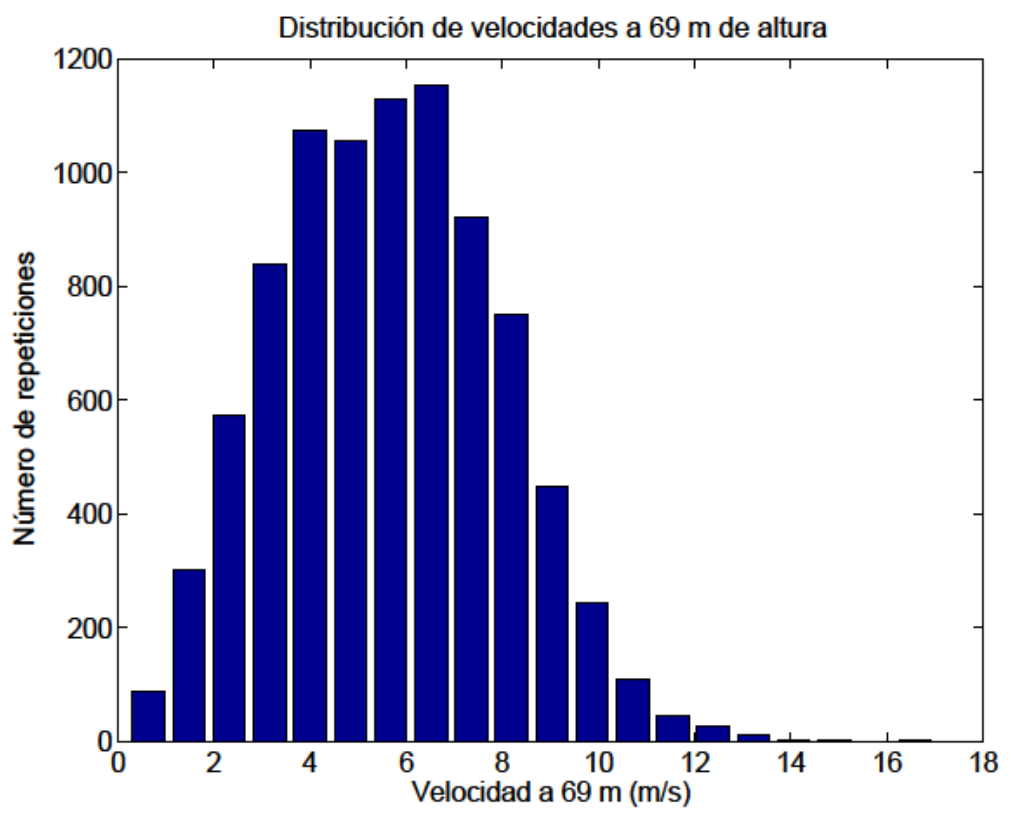

(a) Histograma de velocidades del viento.

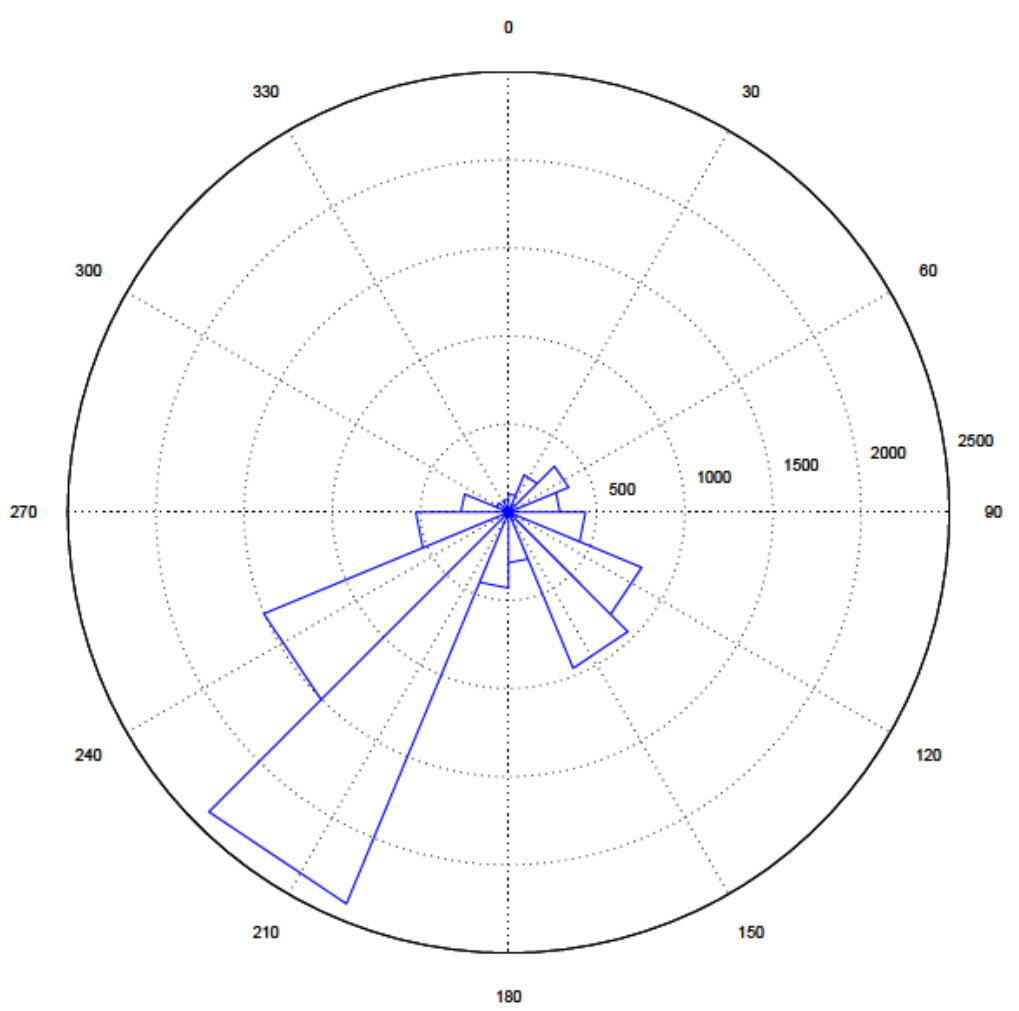

(b) Rosa de los vientos.

Figura 3.17: Características del recurso eólico en el parque eólico 2. 


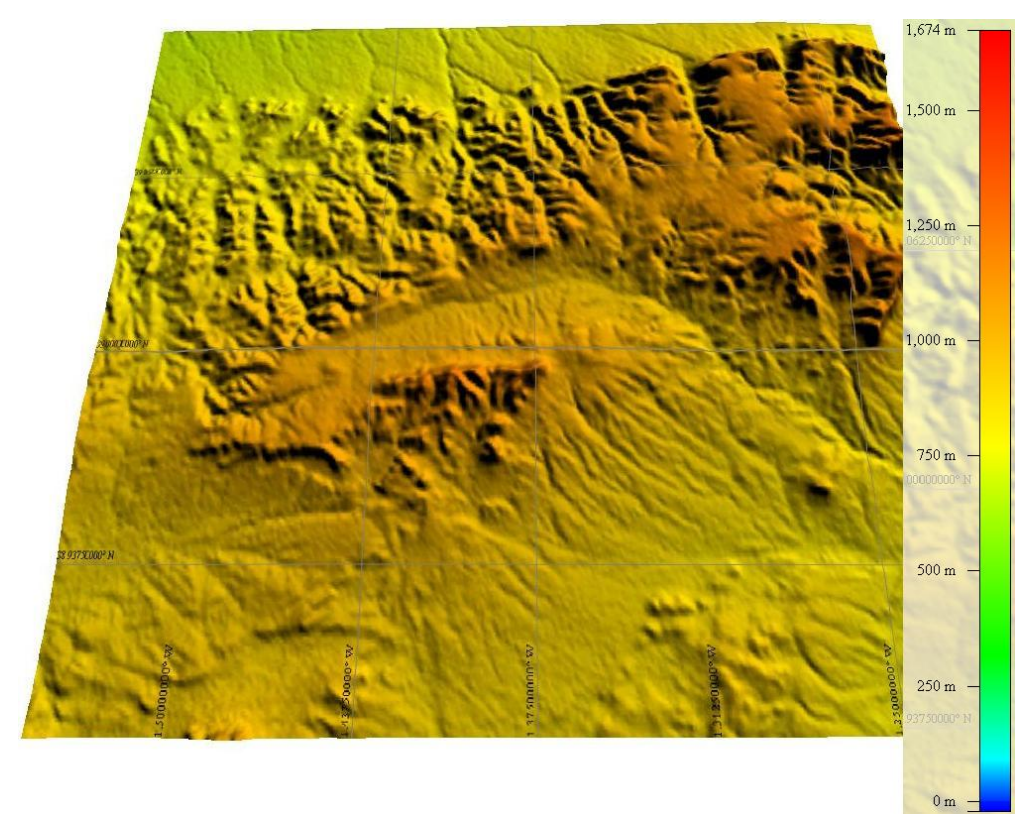

Figura 3.18: Imagen en 3D del emplazamiento 3.

\subsubsection{Parque eólico 3}

El tercer y último parque eólico en el que se ha instalado el equipo LiDAR se encuentra en la localidad de Higueruela, al este de la provincia de Albacete. De hecho, esta región representa una de las zonas con mayor potencia instalada en Castilla-La Mancha (segunda Comunidad Autónoma con mayor potencia instalada en España, tal y como se señaló en el apartado 1.1). La duración del estudio ha sido superior a dos meses; concretamente, desde el día 2 de diciembre de 2011 hasta el 17 de febrero de 2012.

$\mathrm{Al}$ igual que los dos parques anteriores, la orografía de este parque eólico también se puede considerar como compleja. Para visualizar esta circunstancia, la figura 3.18 muestra una reconstrucción topográfica en tres dimensiones, de $40 \mathrm{~km}$ de lado, sobre los alrededores del parque eólico. Dicho parque eólico se sitúa en el centro de la figura 3.18, por lo que se puede ver que se encuentra localizado entre dos relieves montañosos, uno al norte, de mayor extensión y, otro al sur, de menor extensión, justificando así la complejidad de la orografía.

Dado que este parque eólico se han realizado análisis tanto de comparación del equipo LiDAR con respecto a anemometría de góndola, como de la influencia del gradiente de viento en la generación, el WindCube ha sido configurado para medir velocidad y dirección del viento a las siguientes diez alturas: $40 \mathrm{~m}, 50 \mathrm{~m}, 62 \mathrm{~m}, 72 \mathrm{~m}, 85 \mathrm{~m}, 95 \mathrm{~m}, 110 \mathrm{~m}, 140 \mathrm{~m}, 160 \mathrm{~m}, \mathrm{y}$ $200 \mathrm{~m}$. Asimismo, se cuenta con el registro de datos relativos a potencia y 
velocidad del viento en los nueve aerogeneradores del parque eólico a raíz de la información aportada por el sistema "Supervisory Control And Data Acquisition (SCADA)" de dicho parque.

Junto con las características topográficas del emplazamiento, es de vital importancia tener en cuenta la disposición de los equipos sobre el terreno. Para ello, en la figura 3.19 se muestra un plano en dos dimensiones con la distribución de los equipos sobre el terreno. En dicha figura el LiDAR se ha representado con el mismo símbolo que la torre meteorológica y se encuentra entre los aerogeneradores 3 y 4 . Como se puede deducir a raíz de la figura 3.19 , hay una primera hilera de 6 aerogeneradores situados a una distancia inferior a $500 \mathrm{~m}$ del LiDAR; mientras que los 3 restantes forman una segunda hilera localizada a una distancia superior a $1 \mathrm{~km}$.

En relación con la disponibilidad del equipo en este parque eólico, se deberían haber registrado un total de 11.094 datos. Sin embargo, la cantidad de datos registrados por el anemómetro láser asciende a 11.032; por lo que hay 62 datos que no se han podido registrar. Es decir, sobre 10 horas de medida no se han podido realizar, obteniendo una disponibilidad del $99 \%$. Concretamente, en la primera hora de registro se perdió 1 dato, el día 16 de diciembre se perdieron 3 datos. Posteriormente, el día 4 de febrero no se pudo registrar entre las 14 y las 21 horas (se ha comprobado el motivo de esta interrupción prolongada en el registro por parte del LiDAR y se ha observado que coincide con la desconexión de una de las subestaciones a la que vierte energía el parque eólico), y el resto de pérdidas de datos se produjeron de manera aislada los días 5, 8 y 9 de febrero.

Además de las pérdidas puntuales de información señaladas anteriormente, es importante tener en cuenta la disponibilidad de los datos en función de la altura de medida. Bajo este interés, la tabla 3.2 muestra los datos registrados a cada altura, donde puede observarse que en la altura mayor de medida $(200 \mathrm{~m})$ el equipo no pudo realizar 4024 mediciones. Evidentemente, esta circunstancia acaba reduciendo la disponibilidad útil de este tipo de equipamiento, en línea con lo publicado en, Cañadillas et al. (2011); Zubiaur et al. (2012).

Además de los datos recogidos por el equipo LiDAR, también se ha realizado el registro de información de los equipos del parque eólico (aerogeneradores y torre meteorológica). En el caso de los aerogeneradores, se ha registrado potencia y velocidad de viento cada 2 segundos en los nueve aerogeneradores. Por lo tanto, ha sido necesario el cálculo de la media aritmética cada 10 minutos.

Para finalizar el presente apartado y dar comienzo a los resultados, se presenta, un breve análisis del recurso eólico en el parque eólico 3. La figura 3.20 muestra los parámetros más importantes del recurso eólico en la zona estudiada. Por un lado, la figura 3.20a muestra la distribución de velocidades medias a $78 \mathrm{~m}$ sobre el nivel del suelo desde el 1 de diciembre de 2011 


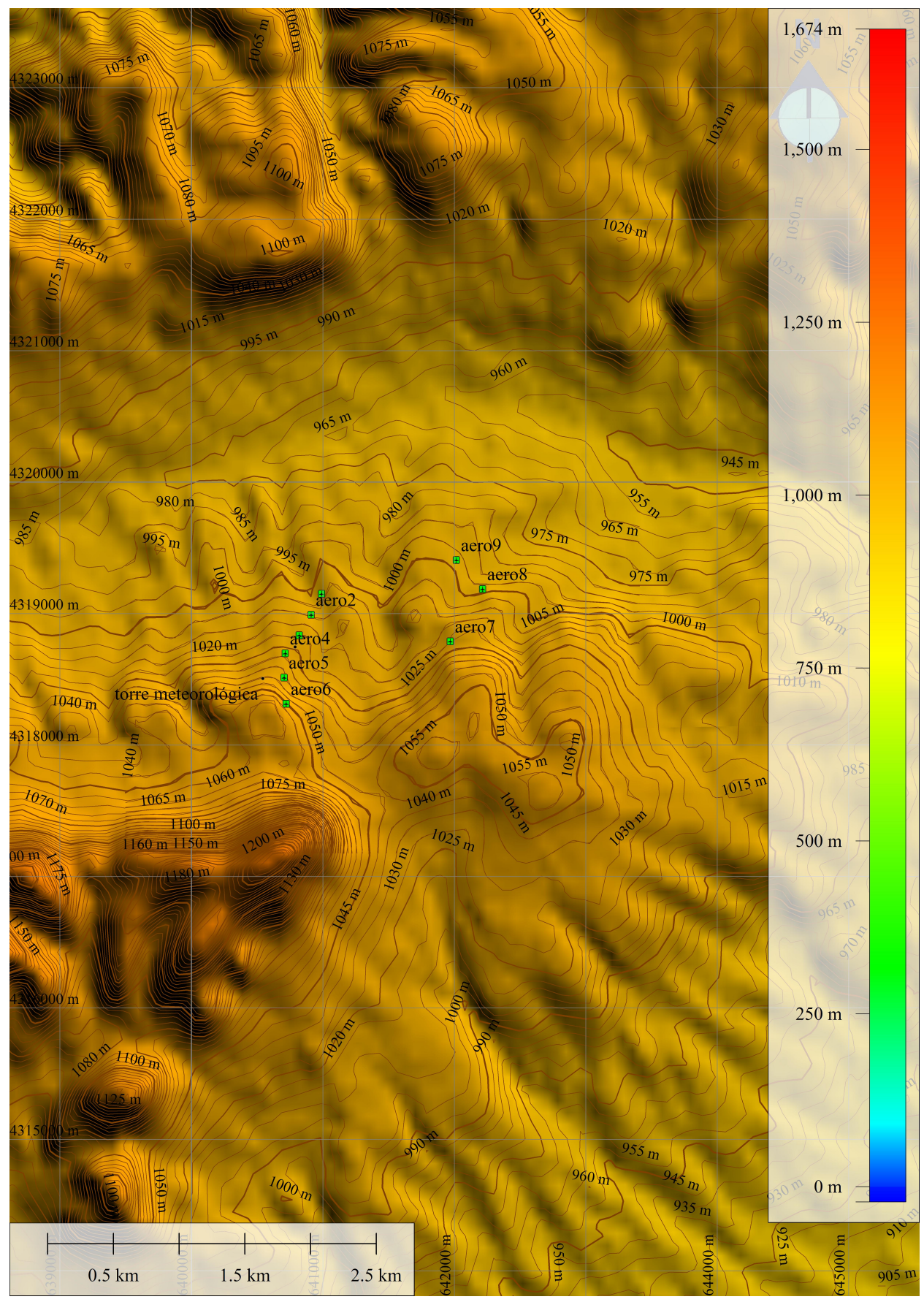

Figura 3.19: Plano en 2D del parque eólico 3. 


\begin{tabular}{c|c}
\hline Altura de medición & Datos disponibles \\
\hline $\mathbf{4 0} \mathbf{~ m}$ & 10964 \\
\hline $\mathbf{5 0} \mathbf{~ m}$ & 10975 \\
\hline $\mathbf{6 2} \mathbf{~ m}$ & 11008 \\
\hline $\mathbf{7 2} \mathbf{~}$ & 10988 \\
\hline $\mathbf{8 5} \mathbf{~}$ & 10974 \\
\hline $\mathbf{9 5} \mathbf{~}$ & 10944 \\
\hline $\mathbf{1 1 0} \mathbf{~ m}$ & 10830 \\
\hline $\mathbf{1 4 0} \mathbf{~}$ & 10033 \\
\hline $\mathbf{1 6 0} \mathbf{~ m}$ & 9140 \\
\hline $\mathbf{2 0 0} \mathbf{~ m}$ & 7070 \\
\hline
\end{tabular}

Tabla 3.2: Cantidad de datos disponible a cada altura de registro del LiDAR en el parque eólico 3 .

hasta el 12 de febrero de 2012 medida por el anemómetro de cazoletas del aerogenerador 3. Mientras que la figura 3.20b representa la sectorización de las direcciones del viento medida por el equipo LiDAR durante el periodo de registro descrito al inicio del presente apartado.

Observando la figura 3.20a, se puede considerar que las velocidades de viento registradas tienen un valor interesante desde el punto de vista de la rentabilidad de un emplazamiento eólico. De manera similar, en la figura $3.20 \mathrm{~b}$ se observa una dirección oeste predominante para el viento incidente sobre el parque eólico; lo que representa una de las características típicas del recurso eólico en esta región de Albacete. 


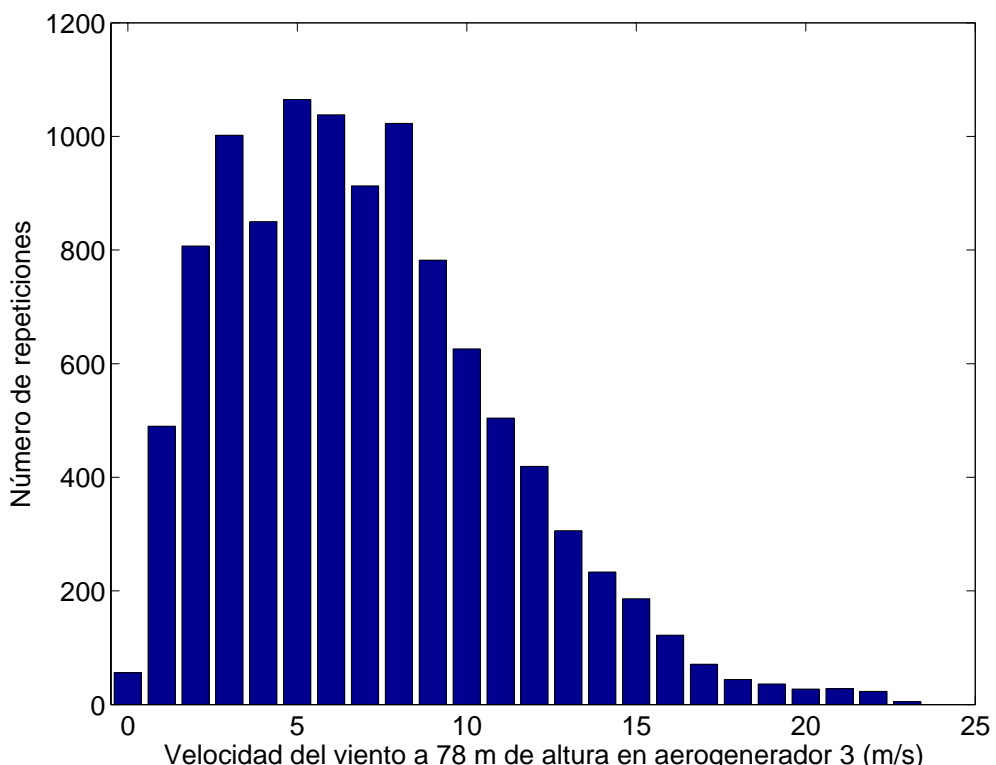

(a) Histograma de velocidades del viento.

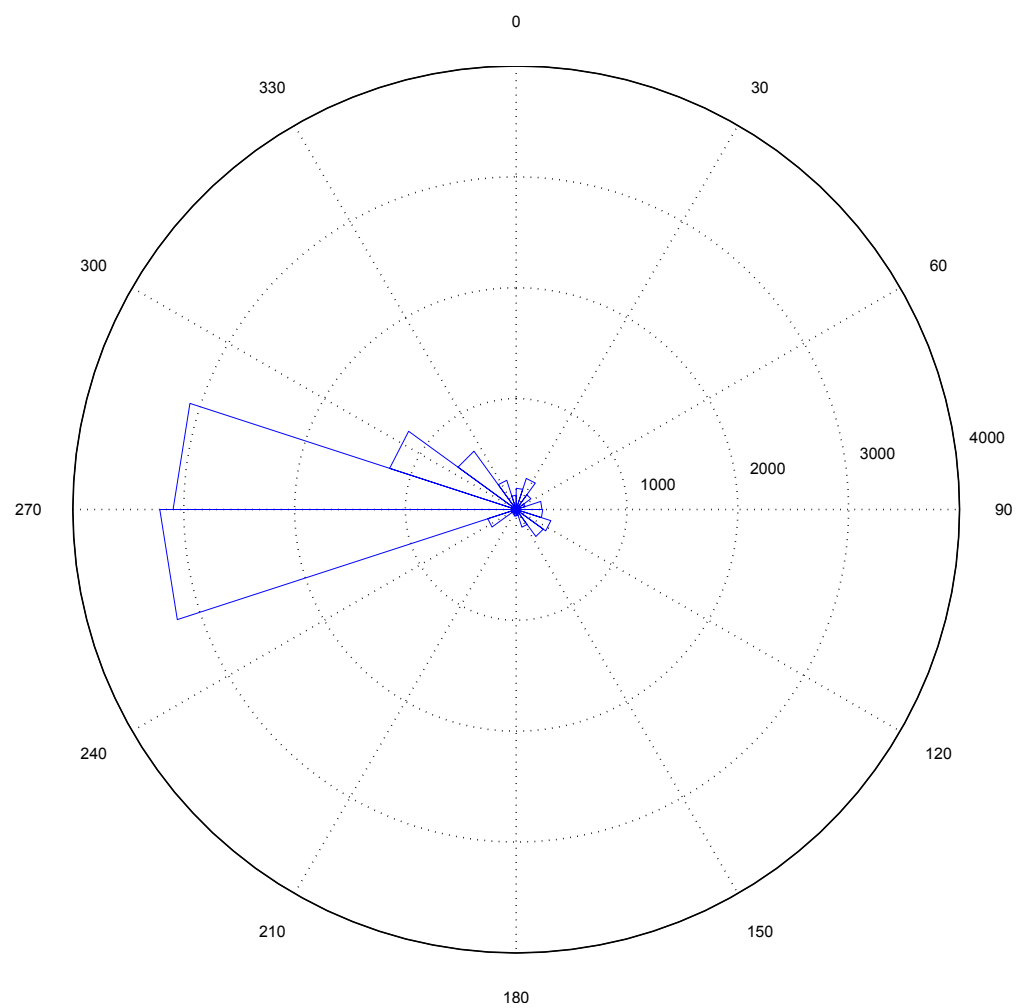

(b) Rosa de los vientos.

Figura 3.20: Características del recurso eólico en el parque eólico 3. 


\subsection{Resultados}

Tal y como se señaló en el apartado 3.1, el objetivo del presente capítulo es el empleo de los sistemas LiDAR de medición del viento para abordar dos temas de elevado interés tecnológico dentro del sector eólico. Por una parte, se presenta un estudio comparativo entre mediciones efectuadas con el equipo de anemometría láser WindCube y mediciones efectuadas con sistemas de anemometría convencional instalados en los parques eólicos descritos en el apartado 3.2. Una vez comprobado el grado de precisión del sistema LiDAR, se realiza la caracterización de la curva de potencia de los aerogeneradores de los parques eólicos. Además, se ha llevado a cabo un breve estudio experimental del gradiente de viento en base a las nociones presentadas en el apartado 3.1 .1

De manera adicional, lo primero que se va a presentar es cómo se ha realizado el tratamiento de la información suministrada por los diferentes equipos instalados en los parques eólicos.

\subsubsection{Tratamiento de la información}

La primera etapa en el tratamiento de la información registrada por el equipo LiDAR es la de filtrado. Por un lado, es necesario tener en cuenta los instantes en que el equipo no registra datos a ninguna altura y, por otro, los momentos en que no es capaz de obtener medidas a determinadas alturas. Este trabajo se ha comentado en el apartado 3.2 para cada uno de los parques eólicos analizados.

Otra tarea, también de relevante importancia, que debe ser realizada antes de obtener resultados es comprobar si existe sincronización entre las medidas registradas por los elementos propios del parque eólico - ya sea la torre meteorológica o los aerogeneradores - y las realizadas por el equipo LiDAR, con el objeto de comprobar que la información ofrecida por cada equipo coincide en el tiempo.

En el caso del parque eólico 3, el equipo LiDAR que se instaló en dicho parque comenzó la campaña de medidas el día 2 de diciembre de 2011 a las 11:10 horas, y finalizó el día 17 de febrero de 2012 a las 12:00. Tal y como se señaló en el apartado 3.2 .3 , en este parque se han obtenido datos de los nueve aerogeneradores cada 2 segundos, siendo necesario el cálculo de la media aritmética cada 10 minutos.

El concepto empleado para sincronizar la información se conoce como "correlación cruzada". Este método mide el grado de similitud entre dos señales; lo que permite determinar el tiempo de retardo entre ambas señales. Con esta intención, una vez obtenida la media aritmética de la información recogida por los aerogeneradores, se ha realizado la correlación cruzada de la velocidad del viento registrada por el aerogenerador 3 (debido a su mayor 
cercanía con el LiDAR) con los medidos por el LiDAR en su cuarta altura de medición, dado que esta altura es la que más se acerca a la del buje de dicho aerogenerador. De esta manera, asumiendo que el comienzo de registro de datos en el equipo LiDAR y el aerogenerador se produce el día 2 de diciembre a las 11:00 (así el primer dato se obtendría a las 11:10), la figura 3.21 presenta el correlograma cruzado. En la parte superior de dicha figura se muestra la correlación cruzada propiamente dicha; mientras que en la inferior un zoom sobre el valor máximo de dicha correlación.

En el eje de abscisas de las dos gráficas de la figura 3.21 se muestran los instantes de tiempo en que coincidirían ambas señales, mientras que el eje de ordenadas representa el grado de correlación. Así, se puede apreciar en la figura $3.21 \mathrm{~b}$ que hay un desfase entre señales igual a 6 instantes, es decir, 1 hora de medidas. Dicho de otra manera, la hora real de comienzo de medidas del equipo LiDAR no fueron las 11:00, sino las 12:00. Con el objeto de comprobar esta afirmación, la figura 3.22 muestra el correlograma cruzado considerando un inicio de registro de medidas a las 12:00. Como se puede apreciar sobre la figura $3.22 \mathrm{~b}$, ajustando el inicio de comienzo de medidas del LiDAR a las 12:00, sí que se obtiene una correcta sincronización entre la información aportada por el LiDAR y por los aerogeneradores.

La sincronización de la información que se acaba de mostrar se ha realizado en los tres parques eólicos en los que se han realizado campaña de medidas. Sin embargo, dado que se trata de un proceso repetitivo que no aporta información relevante, no se muestran los resultados gráficos en los otros dos parques.

Por lo tanto, teniendo en cuenta las anteriores consideraciones, ya se está en condiciones de mostrar los resultados obtenidos en relación con el gradiente de viento, la validación del equipo LiDAR, y la influencia del gradiente en la generación eólica.

\subsubsection{Análisis del gradiente de viento}

El análisis del gradiente de viento vertical realizado en el presente apartado se ha efectuado teniendo en cuenta los datos registrados por el WindCube en los parques eólicos 1 y 3 , apartados 3.2.1 y 3.2.3. respectivamente, en relación con las publicaciones realizadas en Honrubia et al. (2010d c).

La primera imagen que se muestra representa las velocidades del viento registradas por el LiDAR a cada altura durante la campaña de medidas llevada a cabo en los parques eólicos 1 y 3 , figura 3.23 . Como se puede ver en la figura 3.23a, altas velocidades de viento fueron registradas aproximadamente a mediados de cada mes en el parque eólico 1. En relación con el parque eólico 3 , figura $3.23 \mathrm{~b}$, se puede observar que se trata de un parque eólico donde en un periodo ligeramente superior a dos meses se ha superado la velocidad de corte de los aerogeneradores en varias ocasiones. 


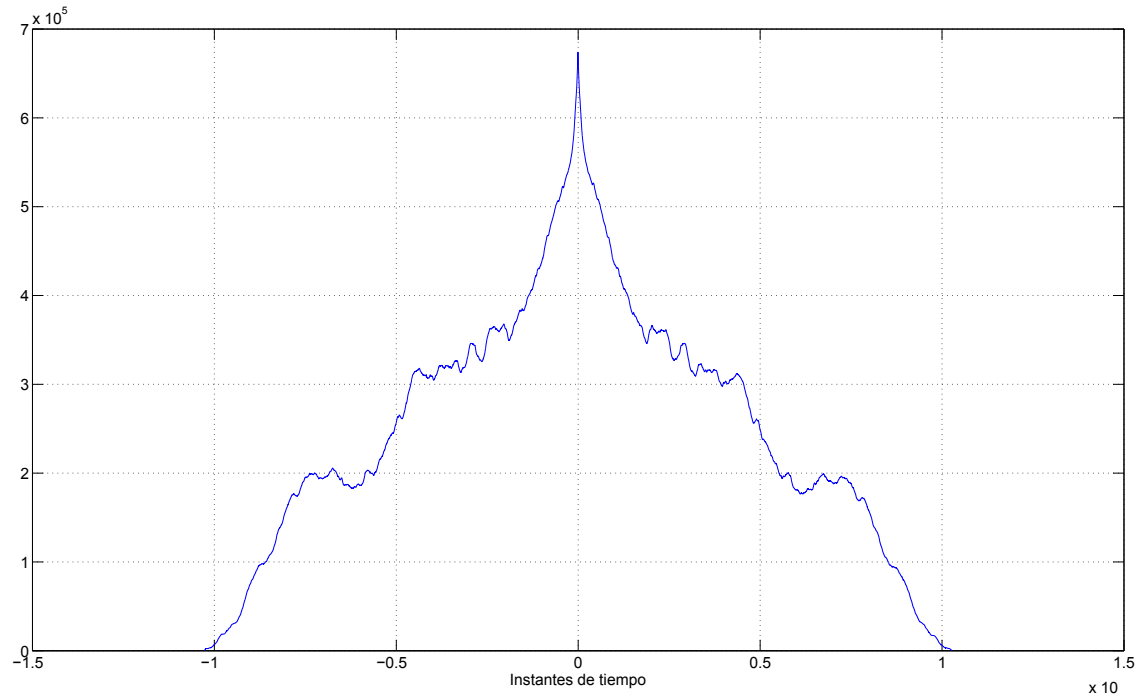

(a) Correlación cruzada asumiendo que el primer dato registrado por LiDAR se produce a las 11:10.

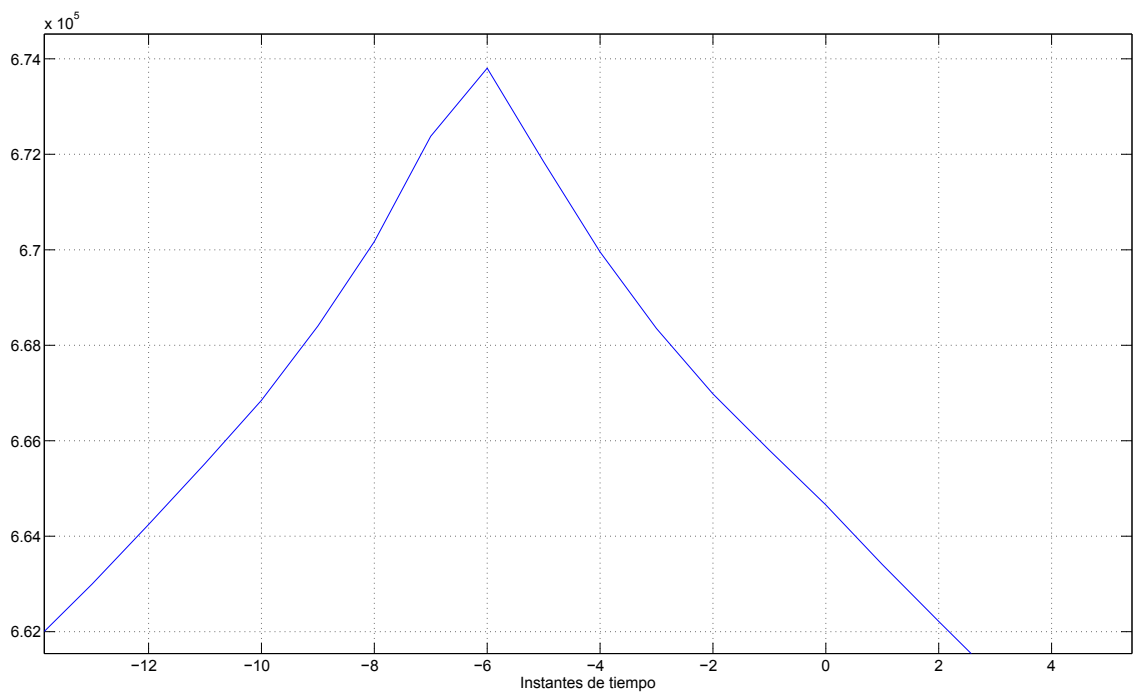

(b) Zoom sobre la correlación (a)

Figura 3.21: Sincronización errónea de la información en el parque eólico 3. 


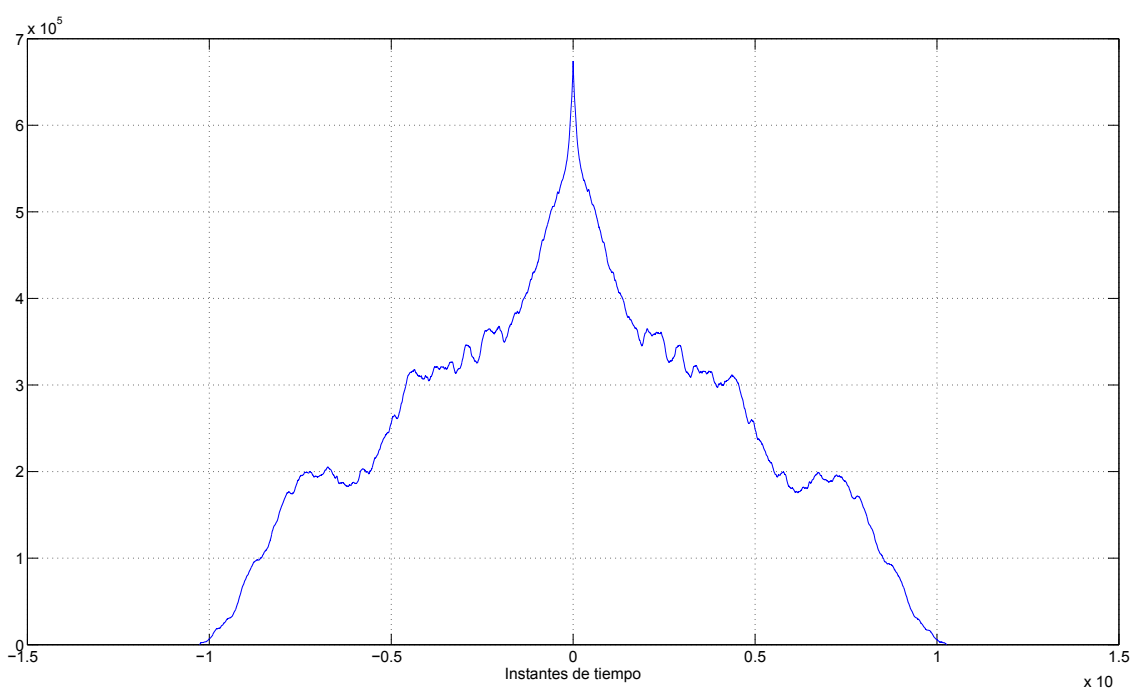

(a) Correlación cruzada asumiendo que el primer dato registrado por LiDAR se produce a las 12:10.

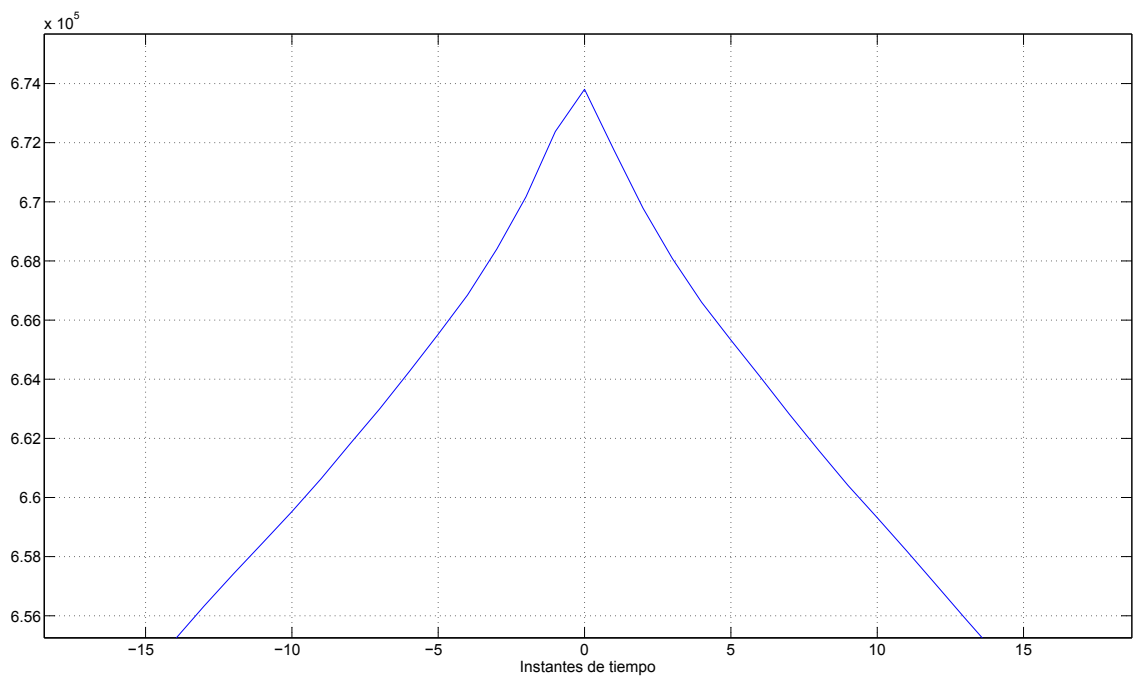

(b) Zoom sobre la correlación (a)

Figura 3.22: Sincronización correcta de la información en el parque eólico 3. 


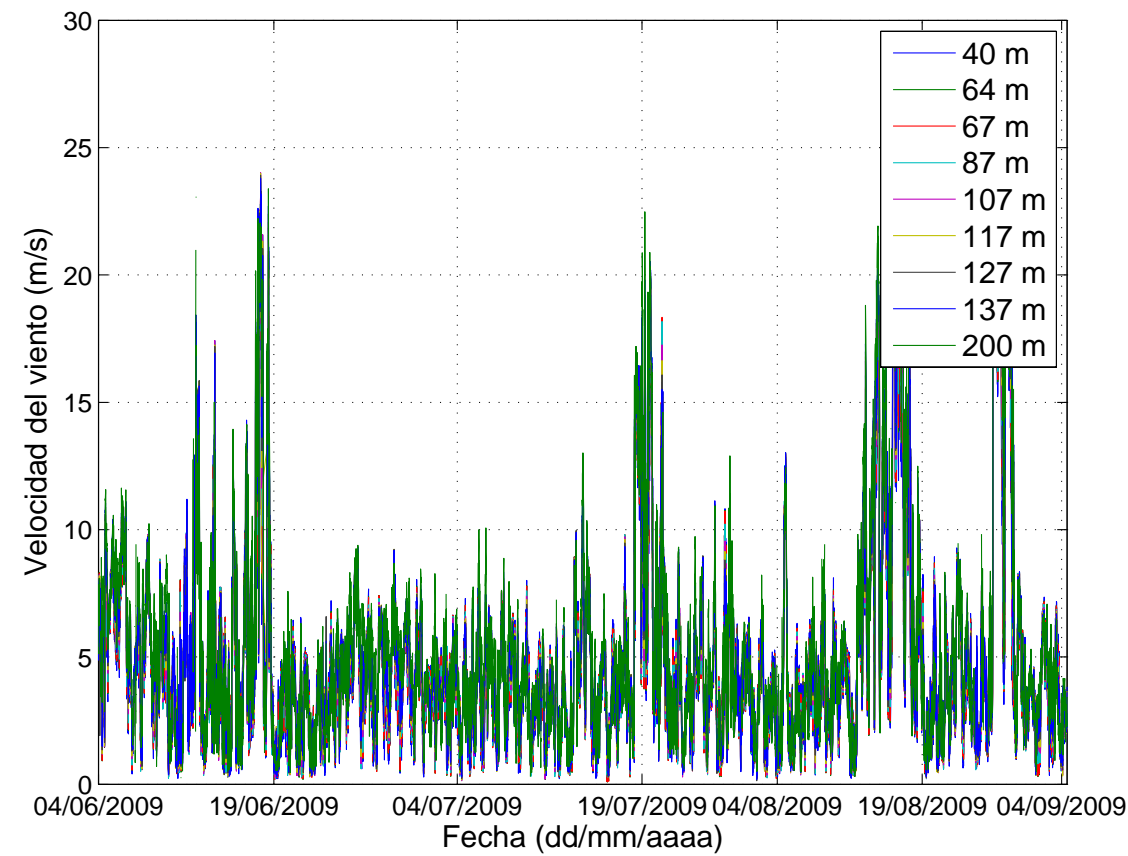

(a) Parque eólico 1.

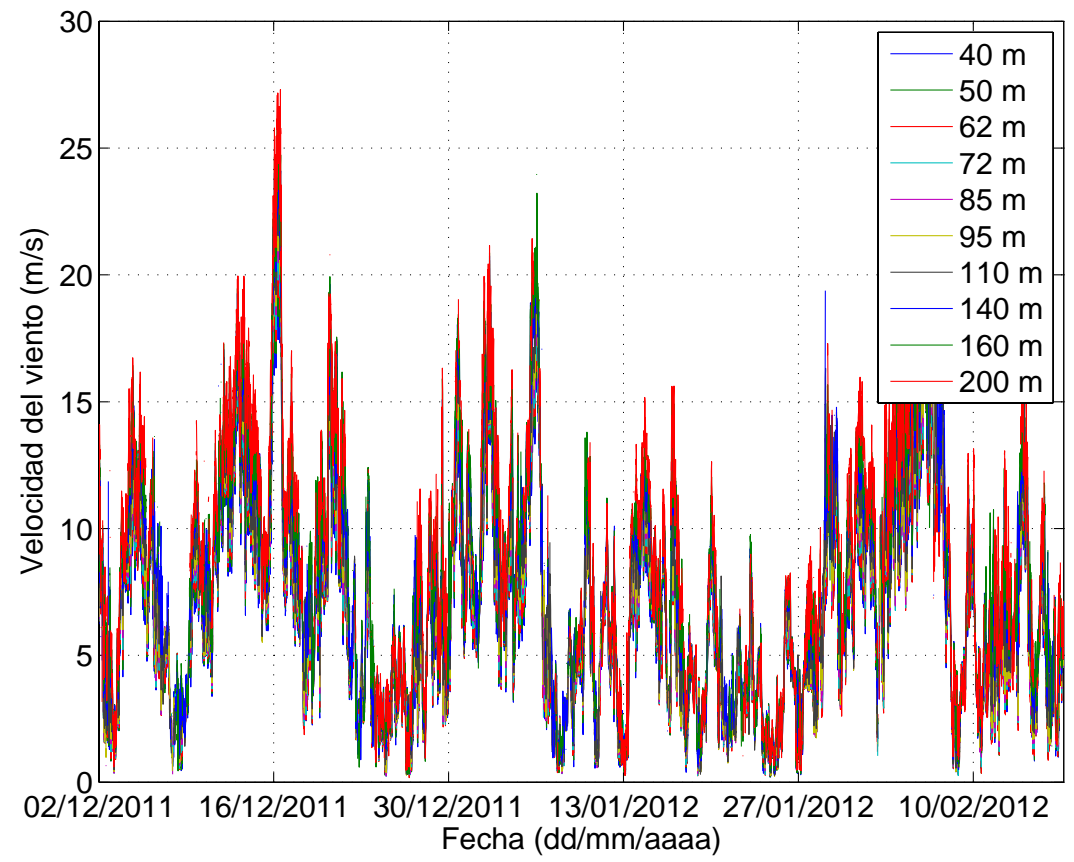

(b) Parque eólico 3.

Figura 3.23: Velocidades de viento registradas por el WindCube en los parques eólicos 1 y 3 . 


\begin{tabular}{c|c|c|c}
\hline Parque $\mathbf{1}$ & Velocidad media & Parque 3 & Velocidad media \\
\hline $\mathbf{4 0} \mathbf{~ m}$ & $4.58 \mathrm{~m} / \mathrm{s}$ & $\mathbf{4 0} \mathbf{~ m}$ & $6.48 \mathrm{~m} / \mathrm{s}$ \\
\hline $\mathbf{6 4} \mathbf{~ m}$ & $4.71 \mathrm{~m} / \mathrm{s}$ & $\mathbf{5 0} \mathbf{~ m}$ & $6.62 \mathrm{~m} / \mathrm{s}$ \\
\hline $\mathbf{6 7} \mathbf{~ m}$ & $4.73 \mathrm{~m} / \mathrm{s}$ & $\mathbf{6 2} \mathbf{~ m}$ & $6.67 \mathrm{~m} / \mathrm{s}$ \\
\hline $\mathbf{8 7} \mathbf{~}$ & $4.85 \mathrm{~m} / \mathrm{s}$ & $\mathbf{7 2} \mathbf{~ m}$ & $6.90 \mathrm{~m} / \mathrm{s}$ \\
\hline $\mathbf{1 0 7} \mathbf{~ m}$ & $4.99 \mathrm{~m} / \mathrm{s}$ & $\mathbf{8 5} \mathbf{~}$ & $7.04 \mathrm{~m} / \mathrm{s}$ \\
\hline $\mathbf{1 1 7} \mathbf{~ m}$ & $5.05 \mathrm{~m} / \mathrm{s}$ & $\mathbf{9 5} \mathbf{~ m}$ & $7.17 \mathrm{~m} / \mathrm{s}$ \\
\hline $\mathbf{1 2 7} \mathbf{~}$ & $5.12 \mathrm{~m} / \mathrm{s}$ & $\mathbf{1 1 0} \mathbf{~ m}$ & $7.48 \mathrm{~m} / \mathrm{s}$ \\
\hline $\mathbf{1 3 7} \mathbf{~ m}$ & $5.18 \mathrm{~m} / \mathrm{s}$ & $\mathbf{1 4 0} \mathbf{~ m}$ & $8.21 \mathrm{~m} / \mathrm{s}$ \\
\hline $\mathbf{2 0 0} \mathbf{~ m}$ & $5.34 \mathrm{~m} / \mathrm{s}$ & $\mathbf{1 6 0} \mathbf{~ m}$ & $8.62 \mathrm{~m} / \mathrm{s}$ \\
\hline- & - & $\mathbf{2 0 0} \mathbf{~ m}$ & $9.34 \mathrm{~m} / \mathrm{s}$ \\
\hline
\end{tabular}

Tabla 3.3: Velocidades de viento medias registradas por el equipo LiDAR en los parques eólicos 1 y 3 .

Las velocidades medias obtenidas a cada altura en ambos parques eólicos se muestran en la tabla 3.3 . En vista de la tabla 3.3 , en el parque eólico 1 se puede considerar que se ha obtenido un gradiente de viento medio con baja rugosidad del terreno o, dicho de otra manera, el grado de inestabilidad atmosférica medio es alto. Sin embargo, en el parque eólico 3, se ha obtenido un gradiente de viento medio con una rugosidad del terreno media o, de manera equivalente, el grado de estabilidad atmosférica medio es alto. Además, la diferencia en la magnitud de las velocidades del viento registradas en cada parque eólico es considerable, en línea con la distribución de velocidades representada en las figuras 3.15a y $3.20 \mathrm{a}$.

De todas maneras, para observar con mayor nitidez los cambios horarios que se producen en la velocidad del viento, la figura 3.24 muestra los perfiles de viento medidos durante el día 5 de junio del año 2009. Se ha elegido este día dentro de los tres meses de duración de la campaña de medidas del parque eólico 1 porque se trata de un perfil representativo del viento en dicho parque eólico.

A raíz de la figura 3.24 se puede ver, sin lugar a dudas, que durante las horas del día, la diferencia en las velocidades del viento es muy pequeña. En el apartado 3.1.1 se comentaron los fenómenos meteorológicos que dan lugar a esta situación. Principalmente, esto es debido a que cuando la temperatura en las proximidades del terreno es mayor que a cotas superiores debido a la acción de la radiación solar, se produce una buena mezcla de las capas atmosféricas, es decir, durante estas horas el aerogenerador se encuentra ante condiciones de atmósfera inestable. Sin embargo, en las horas nocturnas, la 


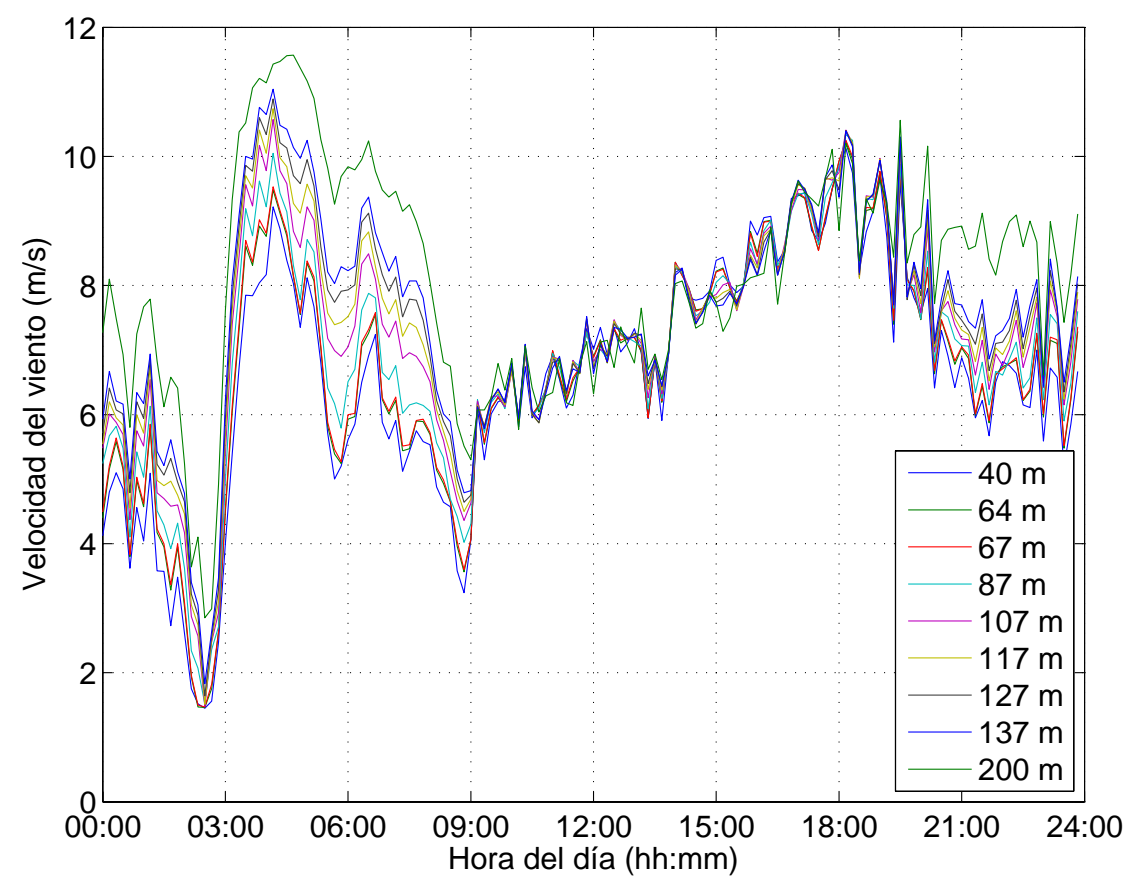

Figura 3.24: Perfil de viento del día 5 de junio de 2009.

distribución de temperaturas se invierte debido al enfriamiento de la tierra, lo que produce que la diferencia entre las velocidades medidas a diferentes alturas se incremente, debido a la situación de atmósfera estable. Este fenómeno se denomina "Estratificación Térmica", Lange y Focken (2005). Es decir, en este parque eólico, durante las horas nocturnas el perfil de viento es mucho más inclinado que durante las horas diurnas.

En línea con lo anterior, analizando la figura 3.24, también resulta interesante observar detenidamente dos perfiles completamente diferentes ocurridos el mismo día, pero con doce horas de diferencia, figura 3.25. En dicha figura puede verse que a $98 \mathrm{~m}$ de altura sobre el terreno se tiene la misma velocidad del viento tanto a las 06:00 horas como a las 12:00; mientras que el perfil a cada una de estas horas es completamente diferente. Tal y como se estudiará en el apartado 3.3.4, estos perfiles de viento influyen sobre la generación de energía.

Para complementar la información aportada por las figuras 3.24 y 3.25 , resulta relevante obtener el perfil horario medio que se ha registrado durante los tres meses de medidas. Así, en la figura 3.26 se muestran los perfiles medios horarios obtenidos a cada hora del día durante los tres meses de registro en el parque eólico 1.

La figura 3.26 aporta dos resultados destacables. Por un lado, se observa cómo mayores velocidades del viento se han registrado durante horas diur- 


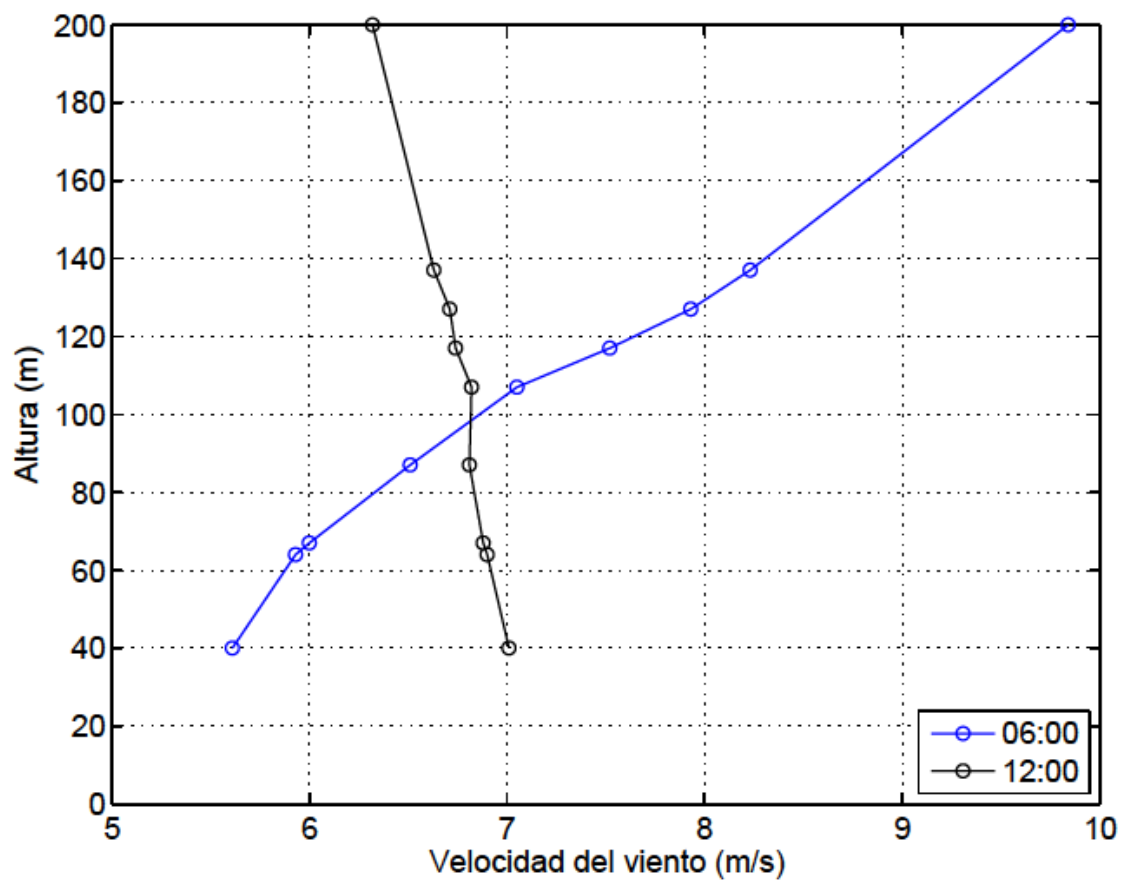

Figura 3.25: Dos perfiles de viento en un mismo día.

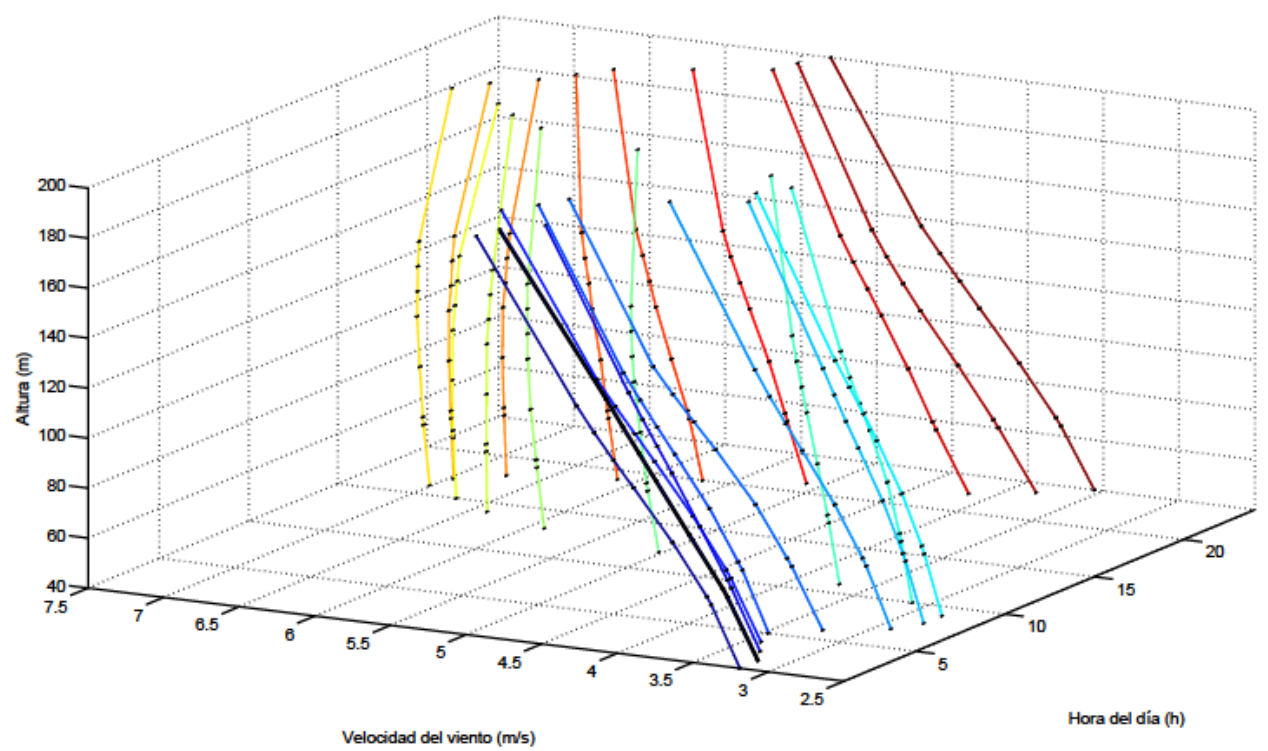

Figura 3.26: Perfiles de viento medios horarios registrados por el WindCube en el parque eólico 1. 


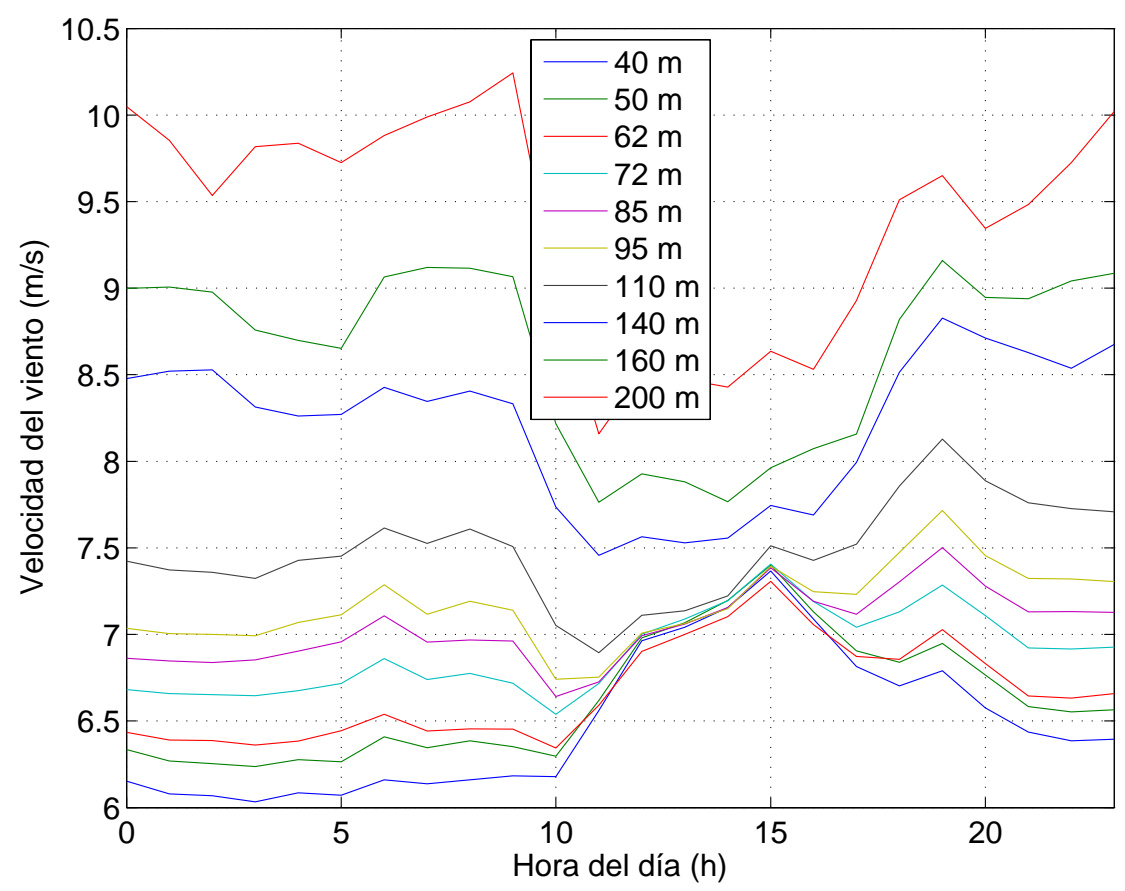

Figura 3.27: Perfiles de viento medios horarios registrados por el WindCube en el parque eólico 3.

nas que durante la noche. Y, por otro lado, perfiles de forma muy variada aparecen a lo largo de las horas del día. Prácticamente, desde las 22 horas hasta las 10 horas de la mañana del día siguiente, los perfiles registrados podrían ajustarse a una Ley Potencial. Sin embargo, los perfiles registrados durante las horas del día poseen una morfología predominantemente plana, con un máximo de velocidad entre los 110 m y 130 m de altura, comenzando a disminuir a después de dicho valor.

De manera similar, la figura 3.27 muestra los perfiles medios horarios encontrados en cada hora del día a cada altura de registro en el parque eólico 3. Se observan características comunes al parque eólico 1, figura 3.26 . ya que el perfil de viento tiene una elevada inclinación durante las horas nocturnas mientras que durante el día es relativamente plano.

Una vez analizados los perfiles de viento registrados en dos parques eólicos situados a unos $500 \mathrm{~km}$ de distancia, se va a continuar estudiando directamente los parámetros de la Ley Potencial, ecuación (3.4). Dado que las velocidades de viento son dato conocido en este ensayo, se van a calcular los diferentes coeficientes de cortadura en función del par de alturas seleccionado, figura 3.28 .

La figura 3.28 muestra el exponente de cortadura de cada par de alturas 


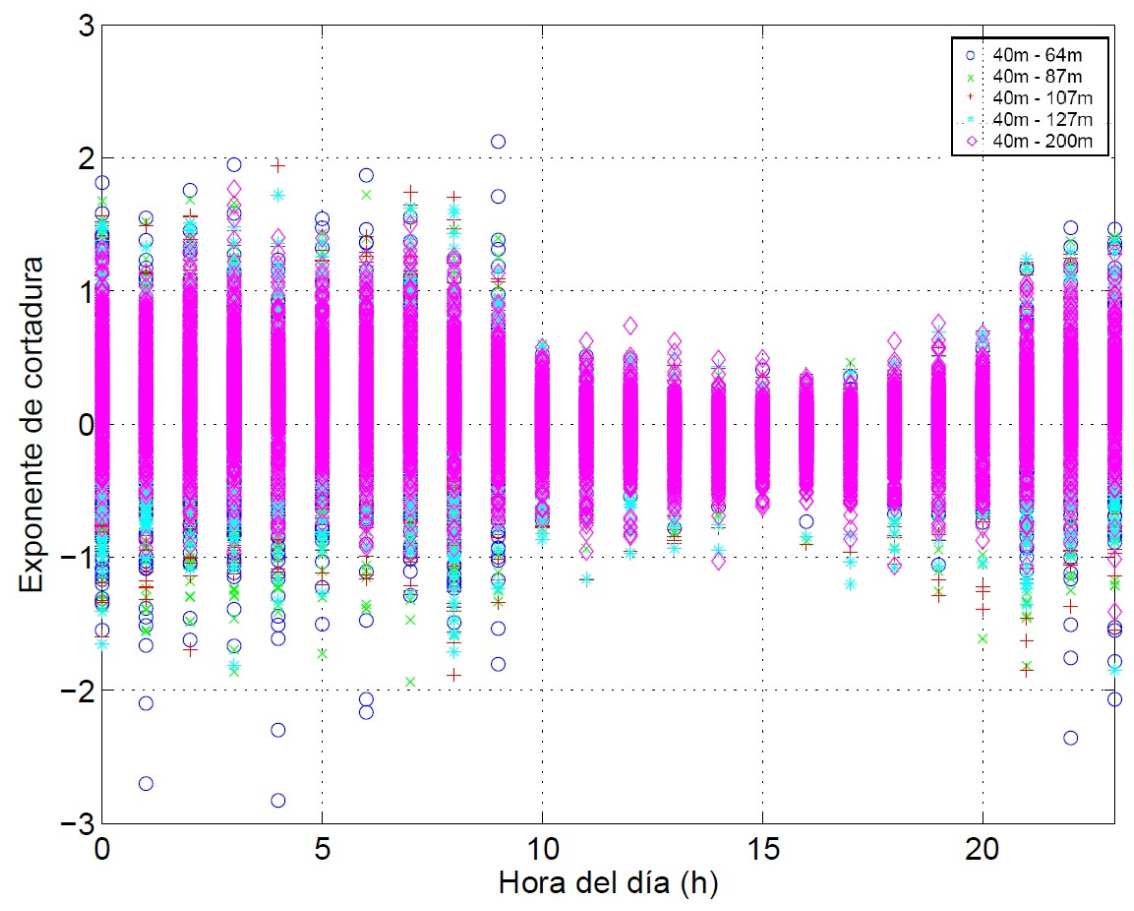

Figura 3.28: Exponentes de cortadura obtenidos en el parque eólico 1 en función del par de alturas. 
en función de la hora del día en el parque eólico 1. En dicho gráfico puede verse que cuanto mayor es la distancia entre pares de alturas, el coeficiente de cortadura obtenido es más reducido. De todas maneras, esta situación no debe ser considerada como característica de cualquier emplazamiento, ya que es completamente dependiente de los perfiles de viento que existan en la zona. Como puede verse, en la figura 3.28 , hay algunos valores de coeficientes demasiado elevados (en valor absoluto). Se ha analizado esta situación y se ha comprobado que estos valores extremos, tanto positivos como negativos, se deben a cálculos realizados cuando las velocidades del viento son reducidas; lo que también ocasiona que el perfil de viento se aleje del teórico.

Nuevamente, igual que se comentó anteriormente para los perfiles, resulta interesante observar las variaciones medias horarias que sufre el exponente de cortadura, figura 3.28 . Se puede comprender que durante las horas del día los coeficientes de cortadura son mucho más pequeños que durante la noche; lo que es coherente con la morfología plana o inclinada de los perfiles de viento, respectivamente, figura 3.26 .

Por lo tanto, después del estudio realizado en el presente apartado, se puede afirmar que los resultados conseguidos sobre los perfiles y el gradiente de viento están en línea con lo publicado por Schwartz y Elliott (2006); Antoniou y Pedersen (2009); Firtin et al. (2011).

\subsubsection{Evaluación de la respuesta del equipo LiDAR frente a sistemas convencionales}

En el presente apartado se encuentra detallado el análisis de la respuesta de esta reciente tecnología en el sector eólico. Debido al interés que existe en el sector en relación con esta temática, se presentan resultados en base a experimentos realizados en los tres parques eólicos, caracterizados en los apartados 3.2.1, 3.2.2, y 3.2.3. Este desarrollo está basado en las publicaciones realizadas en Honrubia et al. (2010a, 2011).

Antes de exponer los resultados, es importante recordar de nuevo que lo que se pretende mostrar es una comparación entre dos métodos de medida completamente diferentes. Concretamente, el equipo de anemometría láser se basa en la suposición de un flujo homogéneo de viento a lo largo de una circunferencia de diámetro creciente con la altura; mientras que los sistemas convencionales - como el anemómetro de cazoletas o el de hélice - se basan en la medición de la velocidad del viento en un único punto.

Teniendo en cuenta las anteriores consideraciones, el objetivo del presente estudio es la correlación de las siguientes variables registradas por ambos equipos:

- Velocidad del viento.

- Desviación estándar en la velocidad del viento. 
- Dirección del viento.

Como métodos de análisis, se van a utilizar, en una primera etapa, el cálculo del "Root Mean Square Error (RMSE)" con el objeto de cuantificar la diferencia entre los valores medidos por los diferentes equipos y, posteriormente, el ajuste por mínimos cuadrados. El RMSE se calcula de acuerdo a la ecuación 3.13.

$$
R M S E=\sqrt{\frac{\sum_{i=1}^{n}\left(V_{\text {WindCube }}-V_{\text {anemo }}\right)^{2}}{N}}
$$

Donde, $V_{\text {WindCube }}$ es la velocidad registrada por el WindCube (en $\mathrm{m} / \mathrm{s}$ ), $V_{\text {anemo }}$ es la velocidad registrada por los anemómetros instalados en las torres meteorológicas (en m/s), y $N$ es el número de medidas consideradas.

Por lo tanto, en los siguientes apartados se detallan los análisis mencionados en las líneas anteriores.

\subsubsection{Efecto sobre la velocidad del viento}

En el presente apartado se va a analizar la velocidad del viento registrada por diferentes tecnologías, en función del parque eólico del que se trate.

En este momento es necesario puntualizar que, mientras no se mencione lo contrario, los resultados obtenidos en el parque eólico 3 emplean la información registrada por el aerogenerador número 3 (debido a su cercanía con LiDAR, ver figura 3.19 y la registrada por el equipo LiDAR en su cuarta altura de medición $(72 \mathrm{~m})$. De esta manera, se comparan velocidades registradas a $78 \mathrm{~m}$ (altura del buje del aerogenerador) con velocidades registradas a $75 \mathrm{~m}$ (altura de medición del LiDAR más su elevación sobre el terreno).

Aplicando (3.13) sobre las campañas de medidas llevadas a cabo en cada parque eólico se obtienen los siguientes resultados:

- Parque eólico 1: RMSE $0.5269 \mathrm{~m} / \mathrm{s}$.

- Parque eólico 2: en función del período de registro, la altura de medición y tipo de anemómetro, el resultado se muestra en la tabla 3.4.

- Parque eólico 3: RMSE $0.5872 \mathrm{~m} / \mathrm{s}$.

Como puede verse en los valores listados anteriormente, el RMSE obtenido en cada parque eólico se puede considerar relativamente reducido. En los parques 1 y 3 , el error es muy parecido, aunque ligeramente superior en el parque 3. Sin embargo, en el parque eólico 2 puede observarse una diferencia considerable entre los resultados medidos por el anemómetro de cazoletas y la anemoveleta; además de un ligero aumento del error a mayor altura. 


\begin{tabular}{l|c|c}
\hline Altura en torre & $\mathbf{4 5} \mathbf{~ m}$ & $\mathbf{6 9} \mathbf{~ m}$ \\
\hline Primer período de registro \\
Thies First Class & $0.2467 \mathrm{~m} / \mathrm{s}$ & $0.2882 \mathrm{~m} / \mathrm{s}$ \\
Young 05103 & $0.2749 \mathrm{~m} / \mathrm{s}$ & $0.4055 \mathrm{~m} / \mathrm{s}$ \\
\hline \multicolumn{3}{|c}{ Segundo período de registro } \\
Thies First Class & $0.5002 \mathrm{~m} / \mathrm{s}$ & $0.5465 \mathrm{~m} / \mathrm{s}$ \\
Young 05103 & $0.4751 \mathrm{~m} / \mathrm{s}$ & $0.6039 \mathrm{~m} / \mathrm{s}$ \\
\hline
\end{tabular}

Tabla 3.4: RMSE calculado en el parque eólico 2.

Además, es importante indicar que el RMSE calculado en el parque eólico 2 es considerablemente superior con el ángulo de escaneo de $15^{\circ}$ que durante el primer período de registro.

Para una mejor comprensión de la similitud entre los valores registrados por los diferentes equipos, se ha calculado el error cuadrático medio en función de la velocidad del viento. De esta manera, se han seleccionado intervalos de $4 \mathrm{~m} / \mathrm{s}$, dentro de los cuales se han calculado los RMSE, resultando las figuras $3.29,3.30,3.31$, y 3.32 . Sobre cualquiera de estas figuras hay tres trazos con diferentes colores. El trazo azul corresponde al cálculo de los RMSE tomando las muestras del WindCube y torre que estén dentro de los límites del intervalo, es decir, se trata del RMSE calculado con menor número de muestras. El trazo negro afecta a los datos registrados por ambos equipos, pero sólo se encuentran dentro del intervalo los medidos por la torre. Y el trazo rojo concierne a todos los datos registrados por ambos equipos, pero sólo se encuentran dentro del intervalo los medidos por el WindCube. La parte superior y central de dichas figuras muestran el RMSE en cada uno de los intervalos, en valores absolutos y relativos, respectivamente; mientras que la parte inferior muestra la cantidad de datos utilizados para cada cálculo. El valor relativo se ha calculado dividiendo el valor absoluto entre la velocidad media registrada en el intervalo.

Observando las figuras $3.29,3.30,3.31$, y 3.32 se pueden extraer algunas conclusiones generales, dado que se han encontrado características comunes en los ensayos realizados sobre tres diferentes emplazamientos. Si sólo se atiende a la imagen superior de las figuras 3.29, 3.30, 3.31, y 3.32 se podría concluir que la diferencia entre mediciones de los equipos no sigue una tendencia definida. Sin embargo, analizando la imagen central de dichas figuras, queda manifiesto que a velocidades de viento reducidas, el error cuadrático medio tiene mayor relevancia que a velocidades de viento elevadas. Este hecho se puede encontrar relacionado, por un lado, con la pérdida de precisión que tiene el anemómetro de cazoletas a bajas velocidades debido a la inercia que acumula en rotación, tal y como se comentó en el apartado 3.1.4. 

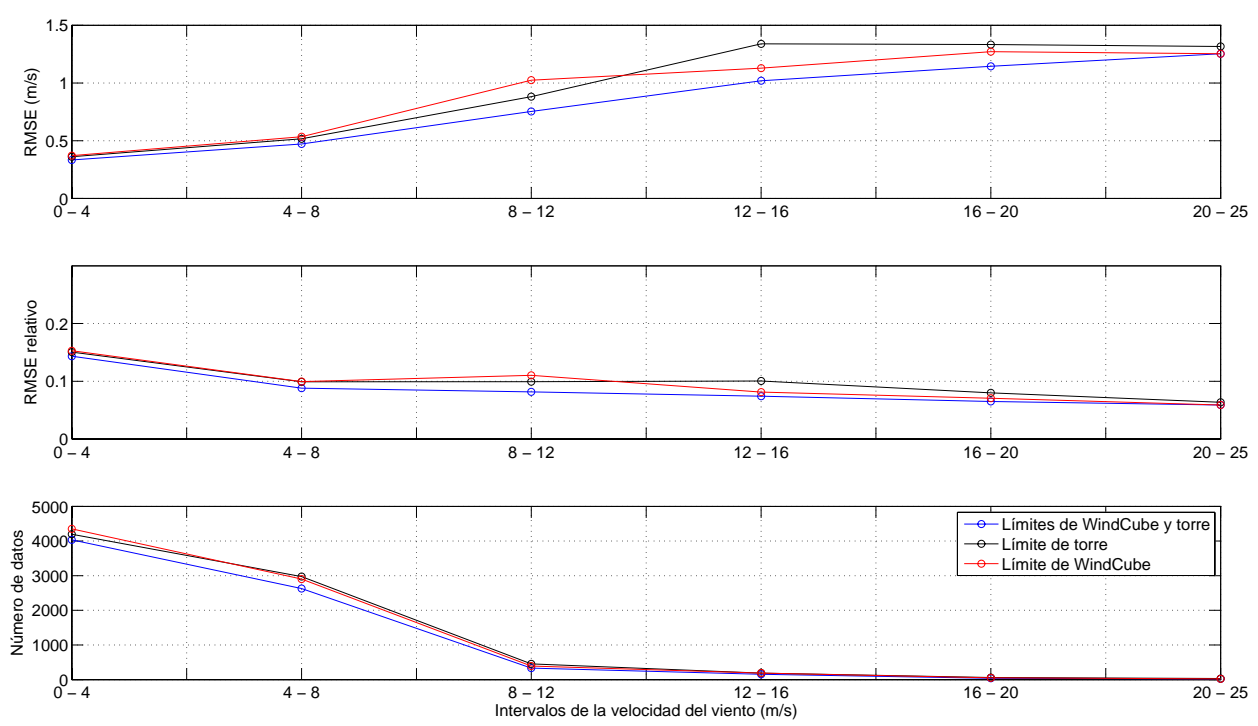

Figura 3.29: RMSE calculado en función de las velocidades del viento en el parque eólico 1.

También, es posible que, debido a la baja velocidad del viento, la dirección del mismo sea variable y, por lo tanto, el anemómetro láser no obtenga una medida precisa de la velocidad del viento. De hecho, se recuerda que cuando el LiDAR de la compañía Natural Power salió al mercado, no era capaz de medir velocidades de viento inferiores a $4 \mathrm{~m} / \mathrm{s}$.

Es importante hacer constar que la diferencia registrada en las mediciones entre equipos a bajas velocidades tiene un carácter menos relevante en el ámbito de la energía eólica, dado que la inmensa mayoría de los aerogeneradores conectados a las redes eléctricas no comienzan a generar energía hasta que la velocidad del viento no ha superado la velocidad de arranque, que se puede situar en torno a los $4 \mathrm{~m} / \mathrm{s}$ para un aerogenerador de $2 \mathrm{MW}$ de potencia nominal.

En el caso del parque eólico 2, figuras 3.30 y 3.31, dado que se registraron velocidades de viento en dos alturas, se puede extraer información adicional. Se observa una ligera reducción del error cuando se efectuaron las medidas a $45 \mathrm{~m}$ de altura en comparación con las de $69 \mathrm{~m}$, en línea con el resultado presentado en la tabla 3.4. Esta situación también ha sido señalada por Gottschall et al. (2012). No se ha incluido el gráfico sobre la comparativa con el anemómetro Young, puesto que se ha comprobado que el resultado es prácticamente idéntico.

Además del estudio del RMSE en función del régimen de velocidades de viento, se ha realizado otro análisis teniendo en cuenta si las diferentes direcciones del viento afectan al cálculo de la velocidad, con las siguientes 

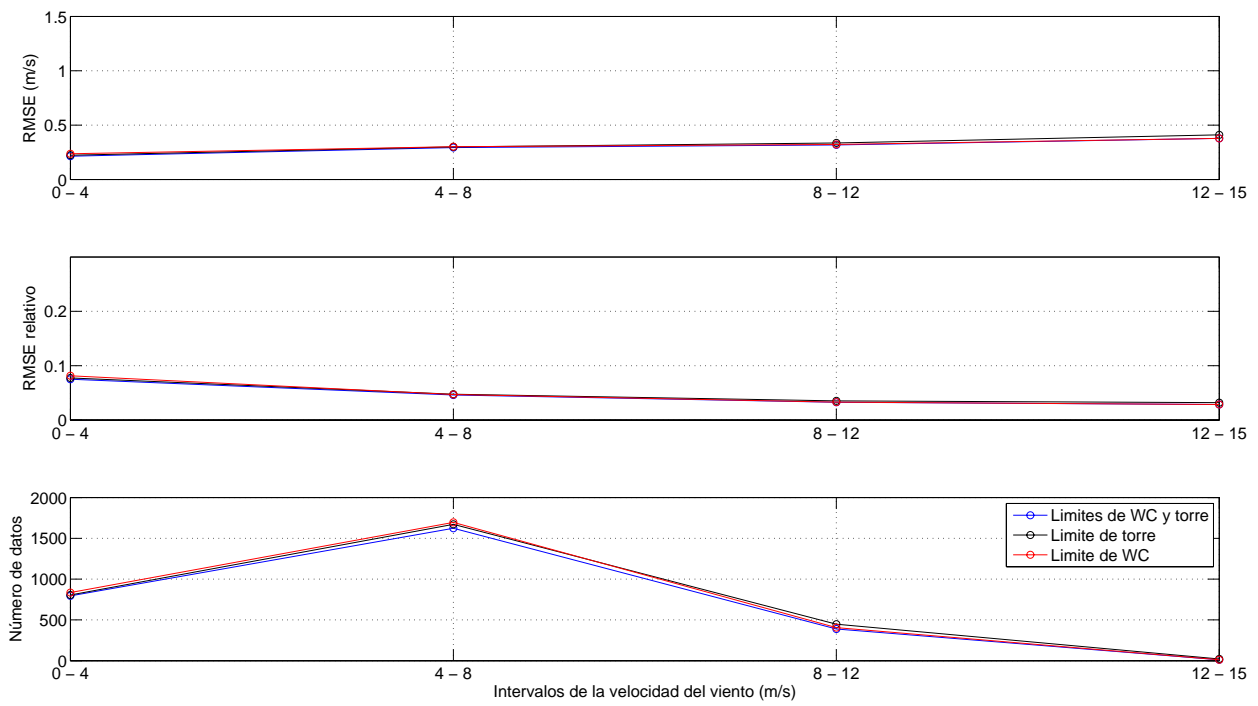

(a) RMSE a $45 \mathrm{~m}$ de altura.
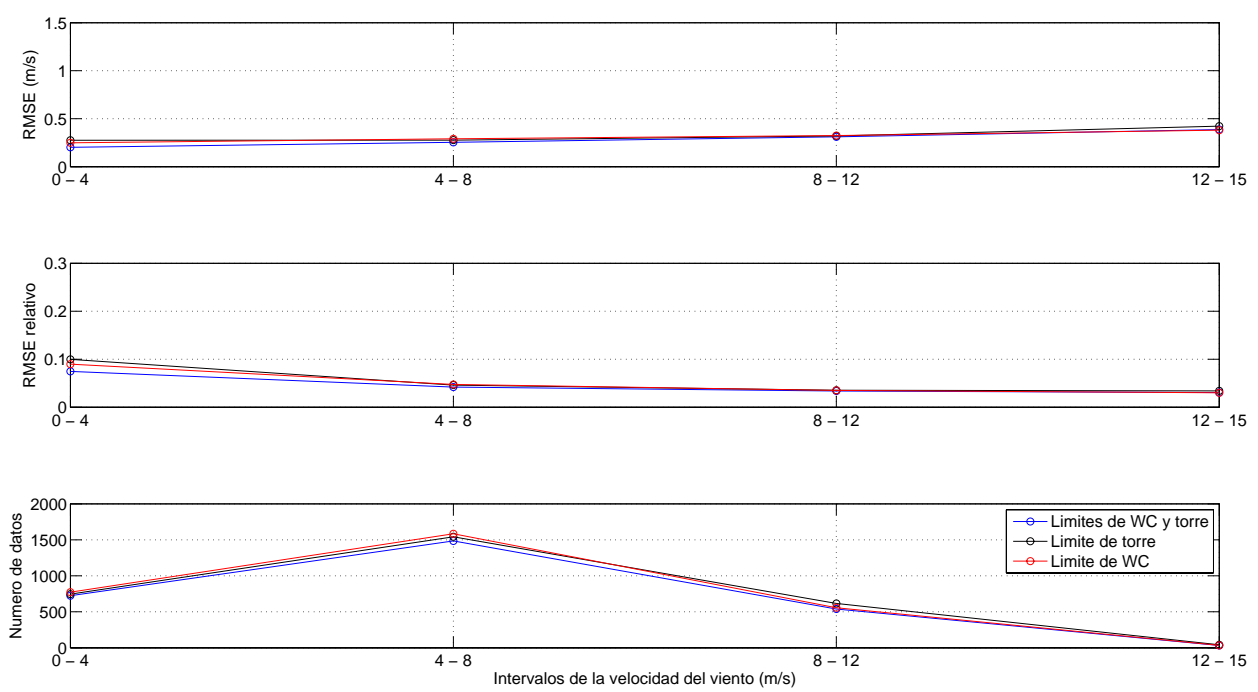

(b) RMSE a $69 \mathrm{~m}$ de altura.

Figura 3.30: RMSE calculado en función de las velocidades del viento en el parque eólico 2 con el anemómetro Thies. Primer período de registro. 

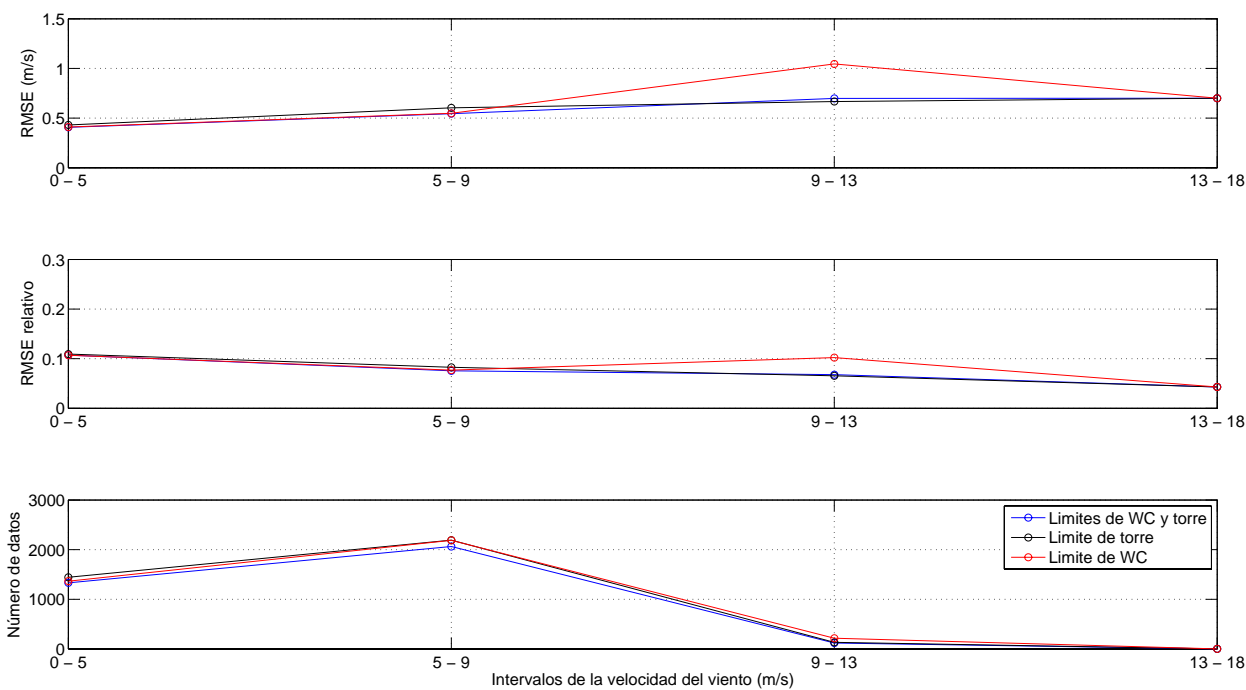

(a) RMSE a $45 \mathrm{~m}$ de altura.
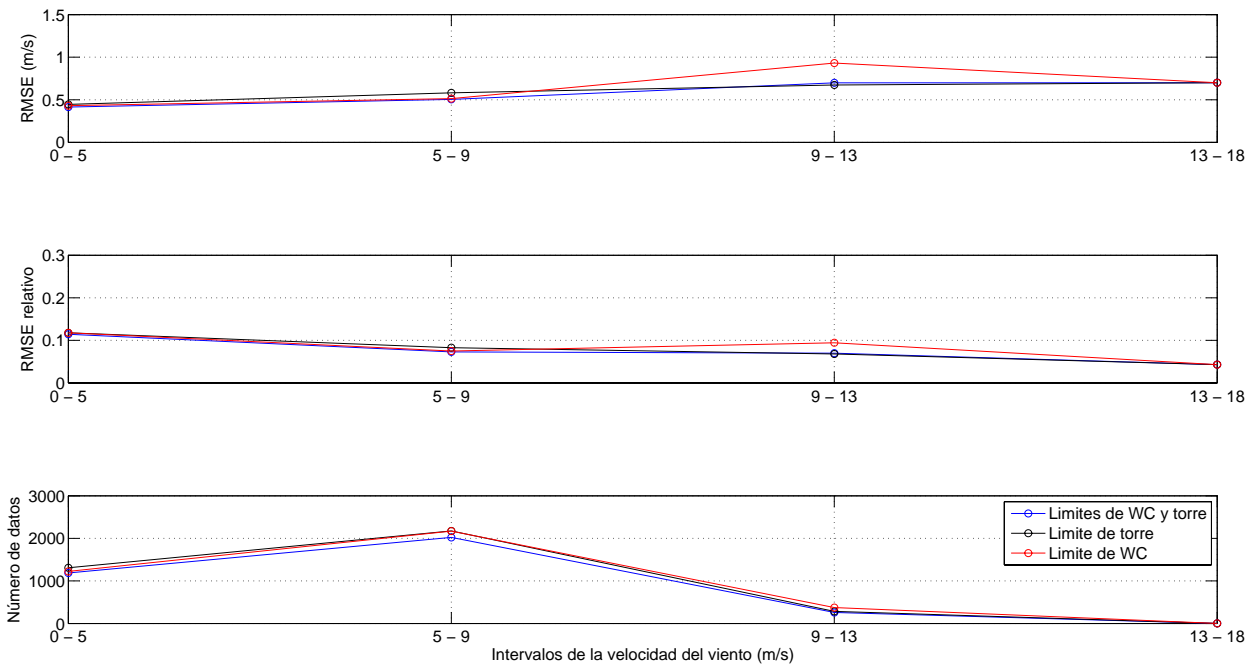

(b) RMSE a $69 \mathrm{~m}$ de altura.

Figura 3.31: RMSE calculado en función de las velocidades del viento en el parque eólico 2 con el anemómetro Thies. Segundo período de registro. 

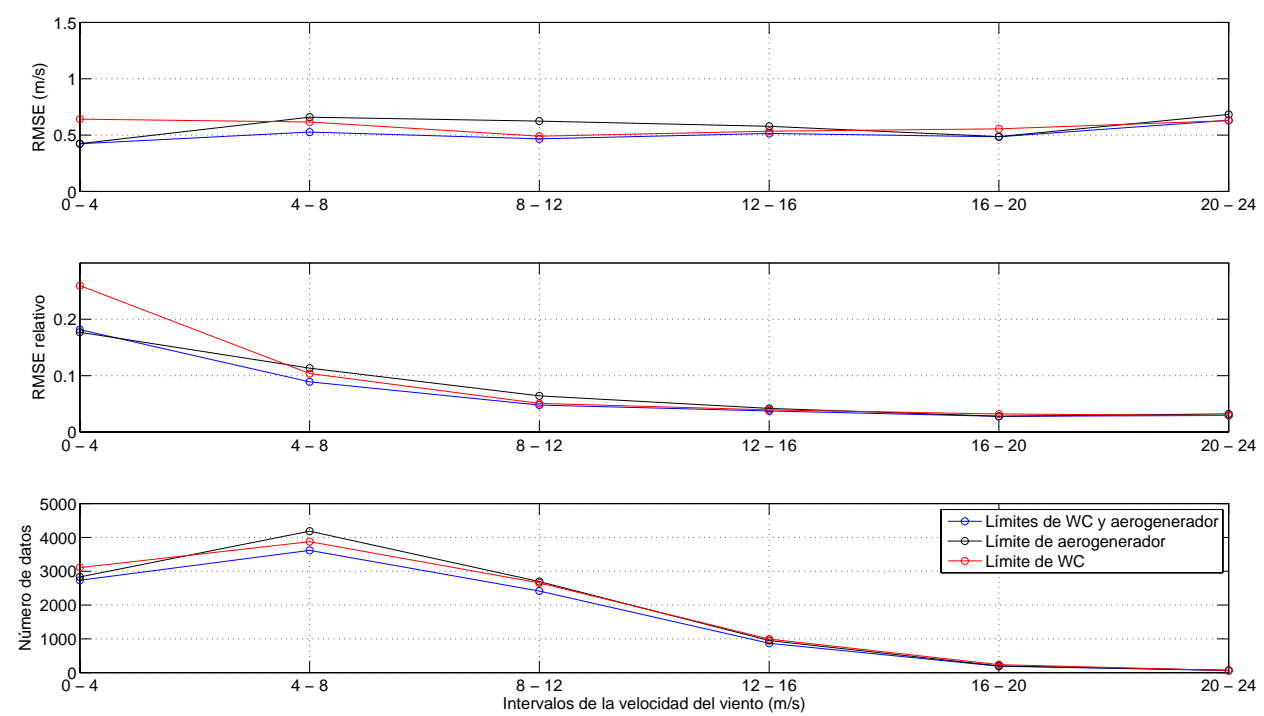

Figura 3.32: RMSE calculado en función de las velocidades del viento en el parque eólico 3.

consideraciones:

- Las direcciones del viento registradas por el LiDAR se han dividido en 6 sectores, empezando por $-30^{\circ}$. Por lo tanto, los sectores resultantes se tienen en la tabla 3.5 .

\begin{tabular}{|c|c|c|c|c|c|}
\hline \multicolumn{6}{|c|}{ Sectores } \\
\hline$-30^{\circ}: 30^{\circ}$ & $30^{\circ}: 90^{\circ}$ & $90^{\circ}: 150^{\circ}$ & $150^{\circ}: 210^{\circ}$ & $210^{\circ}: 270^{\circ}$ & $270^{\circ}: 330^{\circ}$ \\
\hline
\end{tabular}

Tabla 3.5: Sectores de dirección del viento utilizados.

- Con objeto de valorar los resultados obtenidos, en un primer análisis se han utilizado cuatro filtros de velocidad para los parques eólicos 2 y 3 . Inicialmente se han considerado todas las velocidades registradas para, posteriormente filtrar velocidades inferiores a $2 \mathrm{~m} / \mathrm{s}, 4 \mathrm{~m} / \mathrm{s}$, y $6 \mathrm{~m} / \mathrm{s}$.

- Adicionalmente, dado que el parque eólico 3 posee un abundante recurso eólico, también se han incluido filtros de velocidades a $8 \mathrm{~m} / \mathrm{s}$ y a $12 \mathrm{~m} / \mathrm{s}$, para permitir obtener unas conclusiones más consistentes.

- Según el fabricante del equipo LiDAR, éste debe ser alineado de una manera determinada con respecto al norte y, si esta alineación no se realiza adecuadamente, las direcciones del viento registradas por el equipo contarán con error constante. 

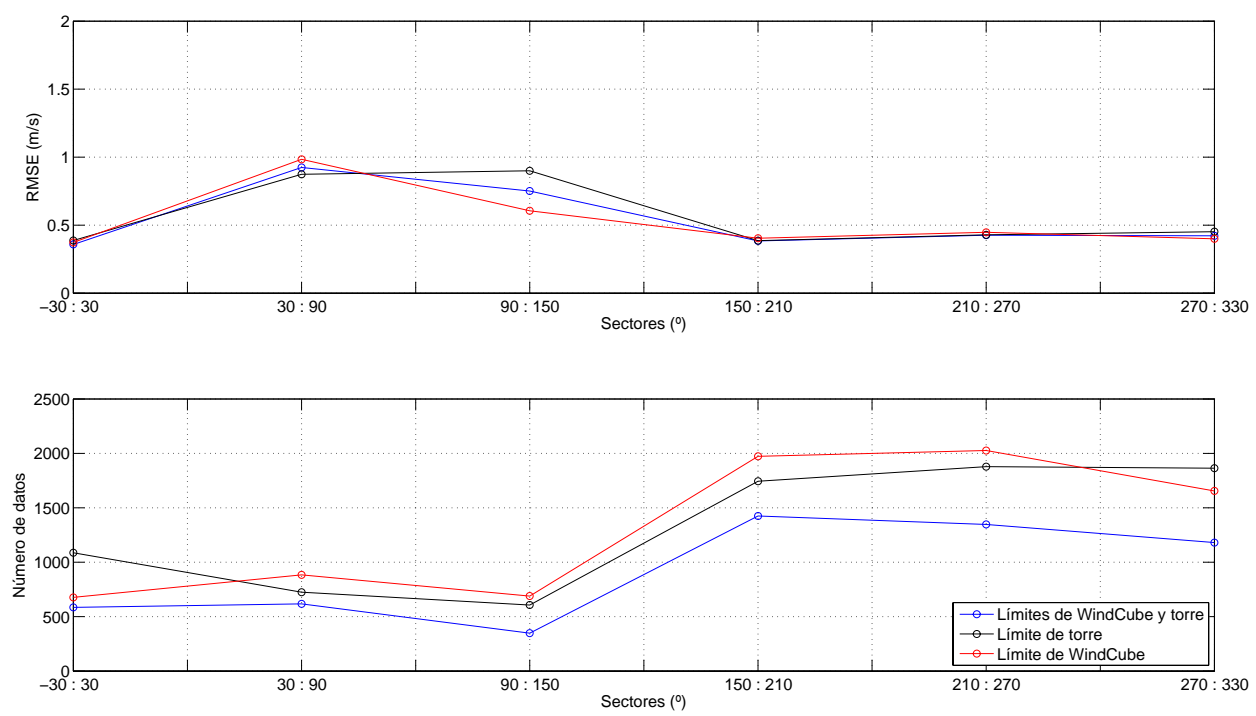

Figura 3.33: RMSE calculado en función de las direcciones del viento en el parque eólico 1.

Para el parque eólico 1, el resultado de esta sectorización se aprecia en la figura 3.33, mostrando en la parte superior el RMSE (en m/s) y en la inferior la cantidad de datos utilizada para cada el cálculo en cada sector. Tal y como se observa en dicha figura, existen dos sectores en los que las velocidades de viento registradas por ambos equipos poseen un error mayor que en el resto de sectores. Este resultado es muy probable que sea debido a que, por un lado, en el sector 3 la torre meteorológica podría influenciar las medidas registradas por el WindCube y, por otro, en esos dos sectores es donde la cantidad de información empleada en el cálculo del RMSE es menor. Concretamente, en Gottschall y Courtney (2010) se recomienda que al menos existan 600 muestras, y se puede ver que en los sectores 2 y 3 este valor resulta comprometido.

De manera similar, en las figuras 3.34 y 3.35 se muestra el análisis del error en el registro de velocidades en función de la dirección del viento para el parque eólico 2. Tal y como se observa en ambas figuras, en los sectores del 1 al 3 el RMSE entre los equipos es mayor que en los sectores del 4 al 6, incluso existiendo una diferencia de más del triple de error para velocidades superiores a $6 \mathrm{~m} / \mathrm{s}$ entre el sector 1 y el 6 . Este resultado es lógico, debido a que en los sectores del 1 al 3 son los sectores en que la torre meteorológica afecta a las mediciones del WindCube, debido a sus posiciones relativas sobre el terreno. Es importante mencionar que el resultado de la línea color magenta en los sectores 1 y 2 no debe ser considerado relevante; ya que el número de datos medidos en esos sectores ha sido de 9 y 21 datos, respectivamente. 

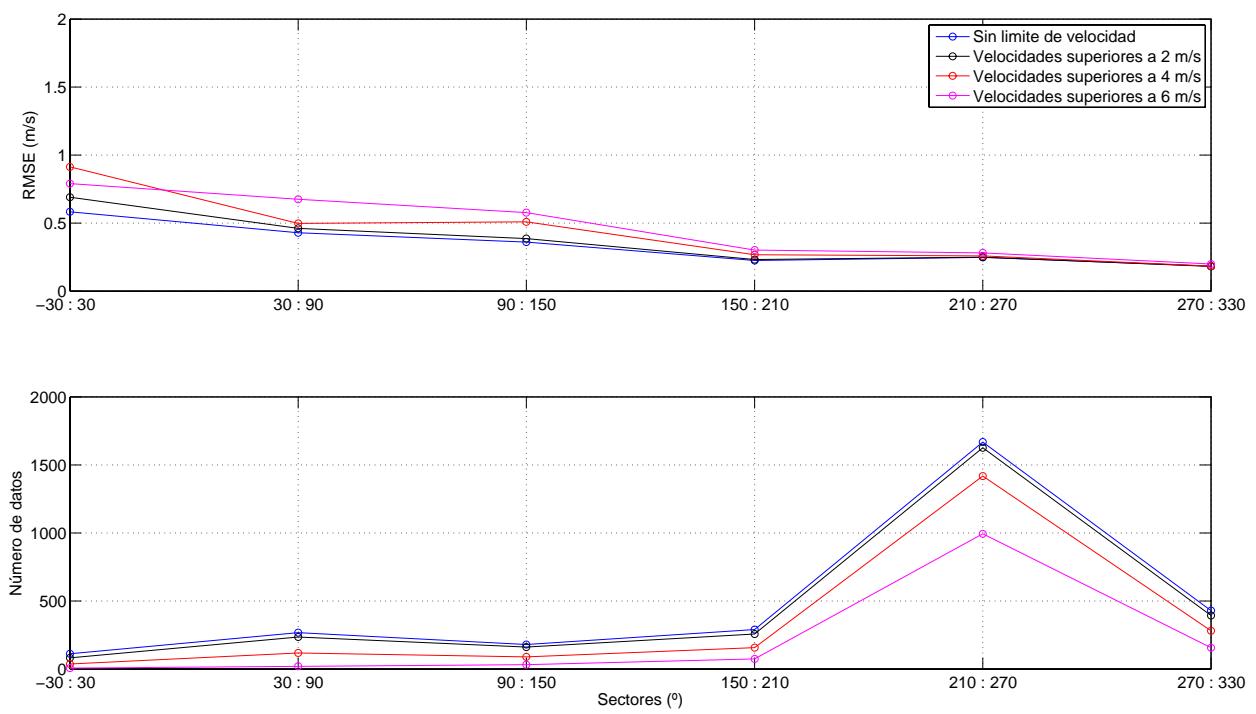

(a) RMSE a $45 \mathrm{~m}$ de altura.
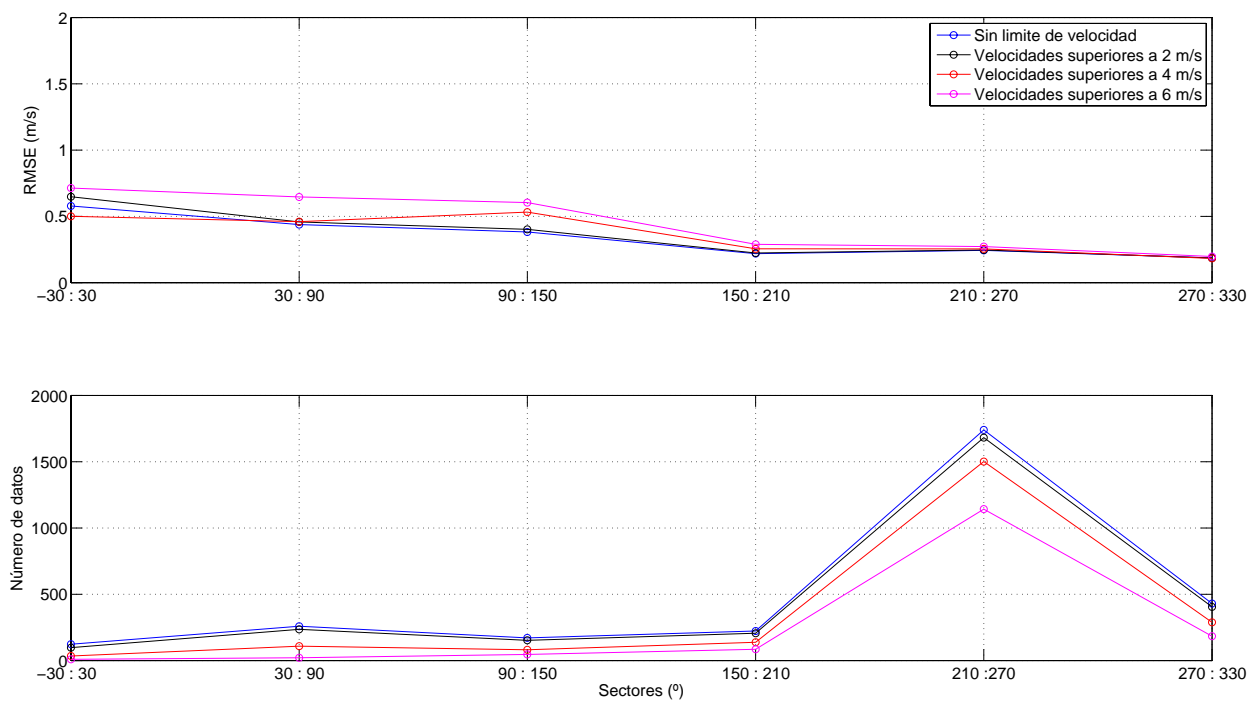

(b) RMSE a $69 \mathrm{~m}$ de altura.

Figura 3.34: RMSE calculado en función de las direcciones del viento en el parque eólico 2, anemómetro Thies. Primer período de registro. 

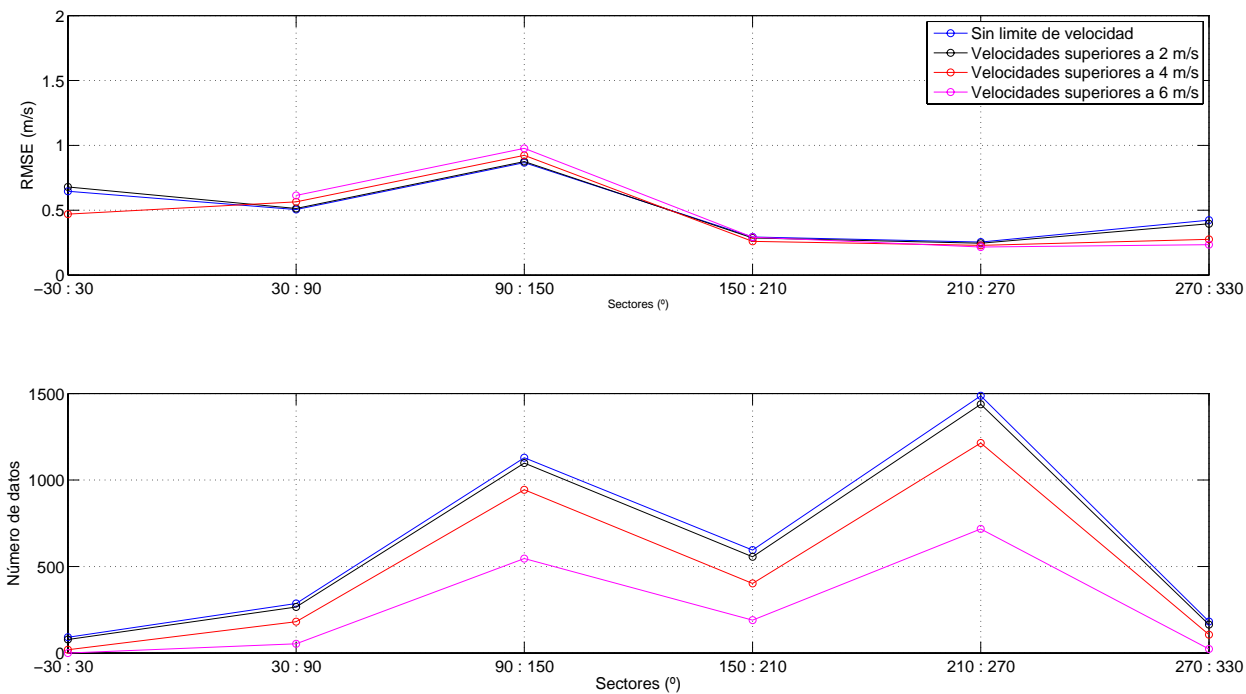

(a) RMSE a $45 \mathrm{~m}$ de altura.
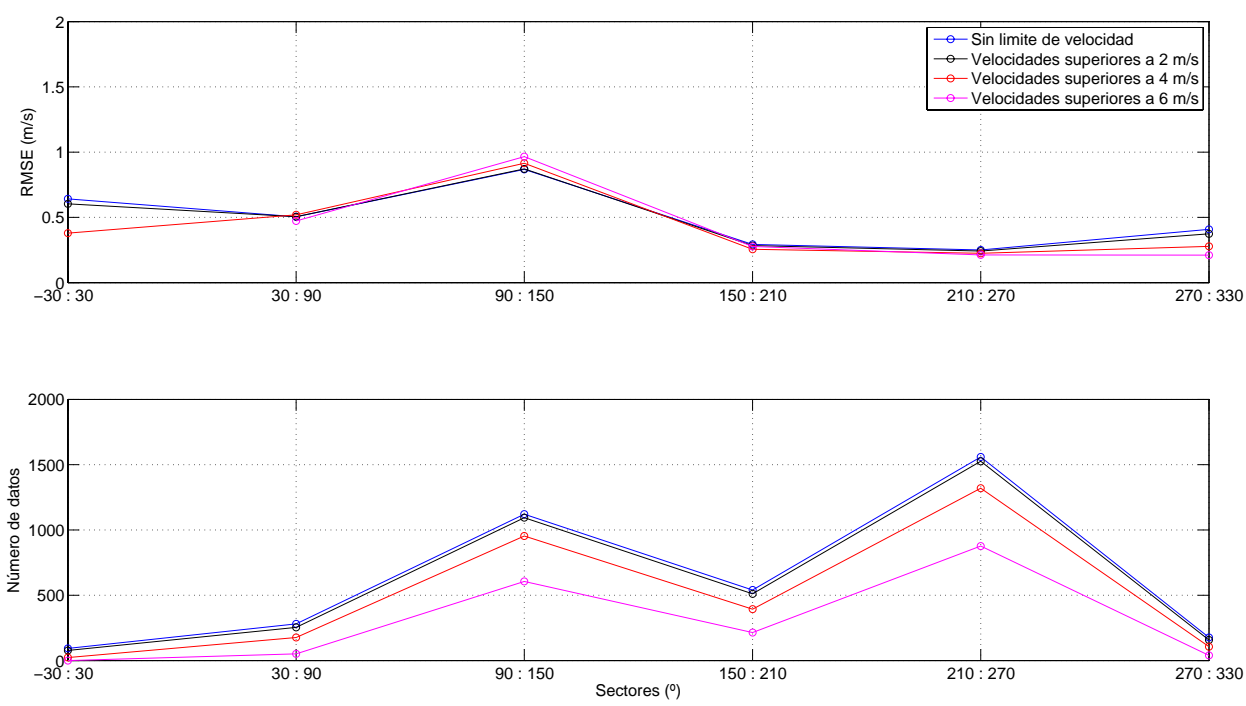

(b) RMSE a $69 \mathrm{~m}$ de altura.

Figura 3.35: RMSE calculado en función de las direcciones del viento en el parque eólico 2, anemómetro Thies. Segundo período de registro. 
Para finalizar el estudio sobre la influencia de la dirección del viento en la medida de las velocidades del mismo, se presenta la figura 3.36 relativa al parque eólico 3. En esta figura se puede observar un error en la velocidad del viento prácticamente constante, a excepción de los sectores 1 y 2 , donde el RMSE entre los equipos es mucho mayor que en el resto de sectores. De hecho, se ha detectado un una diferencia de más de cinco veces en el RMSE entre el sector 1 y el sector 3 para velocidades superiores a $4 \mathrm{~m} / \mathrm{s}$. Se puede señalar que el resultado mostrado en la figura 3.36 era esperable, debido a que los sectores 1 y 2 son los sectores donde el aerogenerador 3 influye de manera directa sobre las mediciones efectuadas por el LiDAR, debido a sus posiciones relativas sobre el terreno (ver figura 3.19). Además, es importante mencionar que el resultado obtenido en los sectores del 1 al 4 no puede ser considerado como definitivo, dado que se observa en ambas figuras que la cantidad de datos utilizados para el cálculo del RMSE es considerablemente inferior al existente en los sectores 5 y 6 . Como puede verse, este último resultado también es análogo con la rosa de vientos obtenida en este parque eólico, mostrada en la figura $3.20 \mathrm{~b}$.

Teniendo en cuenta el error cometido debido al efecto de sombra del aerogenerador 3 sobre el equipo LiDAR en el parque eólico 3 , se ha recalculado el RMSE de todo el intervalo de medidas pero, eliminando las muestras pertenecientes a los sectores 1 y 2 . De esta manera, se obtiene un RMSE de $0.4780 \mathrm{~m} / \mathrm{s}$; lo que representa una considerable reducción del error — próxima al $20 \%$ - con respecto a los $0.5872 \mathrm{~m} / \mathrm{s}$ registrados previamente teniendo en cuenta todo el rango de direcciones del viento. También, con el objeto de aclarar este resultado, en la figura 3.37 se muestra el RMSE en función de las velocidades del viento en aquellas direcciones que no entran dentro de los sectores 1 y 2 . Puede verse sobre dicha figura que, aunque el valor RMSE obtenido es ligeramente inferior al de la figura 3.32 , las conclusiones que se pueden obtener son exactamente las mismas.

Con todo lo anterior, los resultados que se muestren a continuación para el parque eólico 3, implementarán el filtro de direcciones de los sectores 1 y 2, es decir, se eliminará la información registrada en dichos sectores, de la misma manera que se ha realizado en la figura 3.37 .

Una vez realizados los anteriores análisis sobre el error cuadrático medio encontrado en la monitorización de las velocidades del viento, se va a obtener un ajuste por mínimos cuadrados de la información medida por los diferentes equipos. Igual que se desarrolló anteriormente en el cálculo del RMSE, se realiza la correlación de la velocidad del viento registrada por ambos equipos en función de las direcciones del viento. Se ha seleccionado la misma sectorización que el análisis anterior (ver tabla 3.5. El resultado del ajuste por mínimos cuadrados para el parque eólico 1 se muestra en la figura 3.38. Sobre la nube de puntos, se ha ajustado la función lineal que refleja la relación entre las velocidades registradas por ambos equipos. 

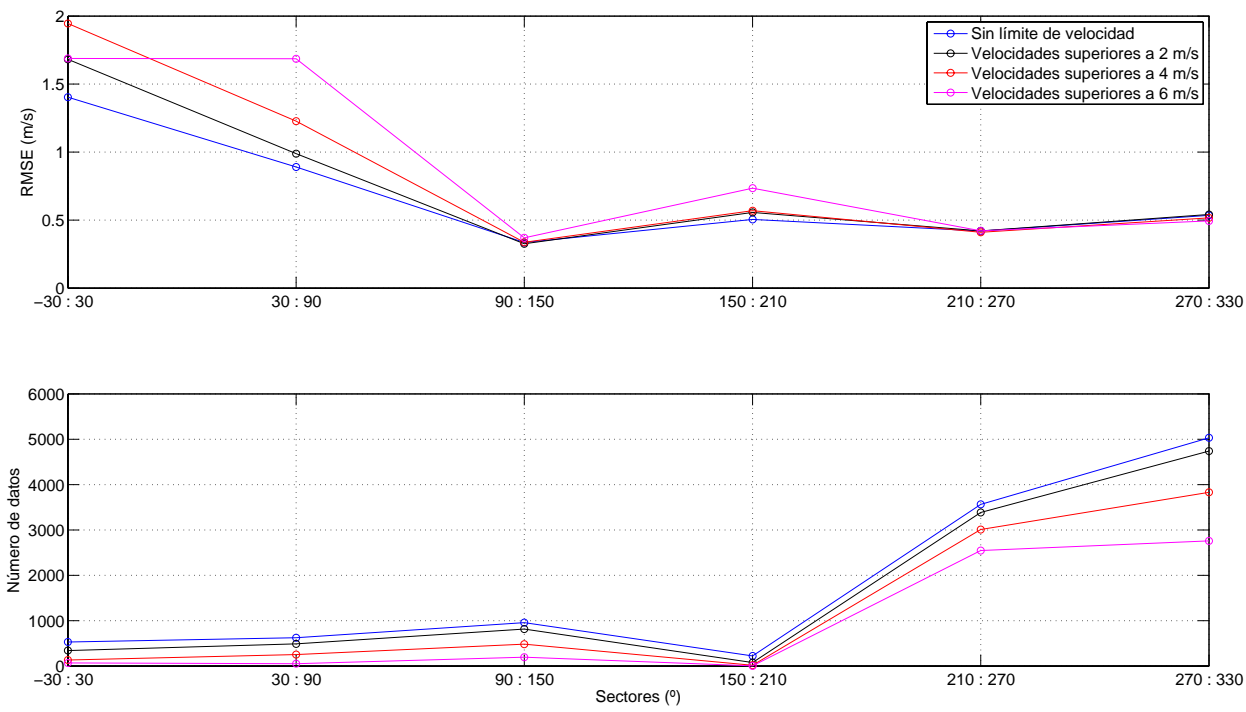

(a) Filtro de velocidades medias de viento
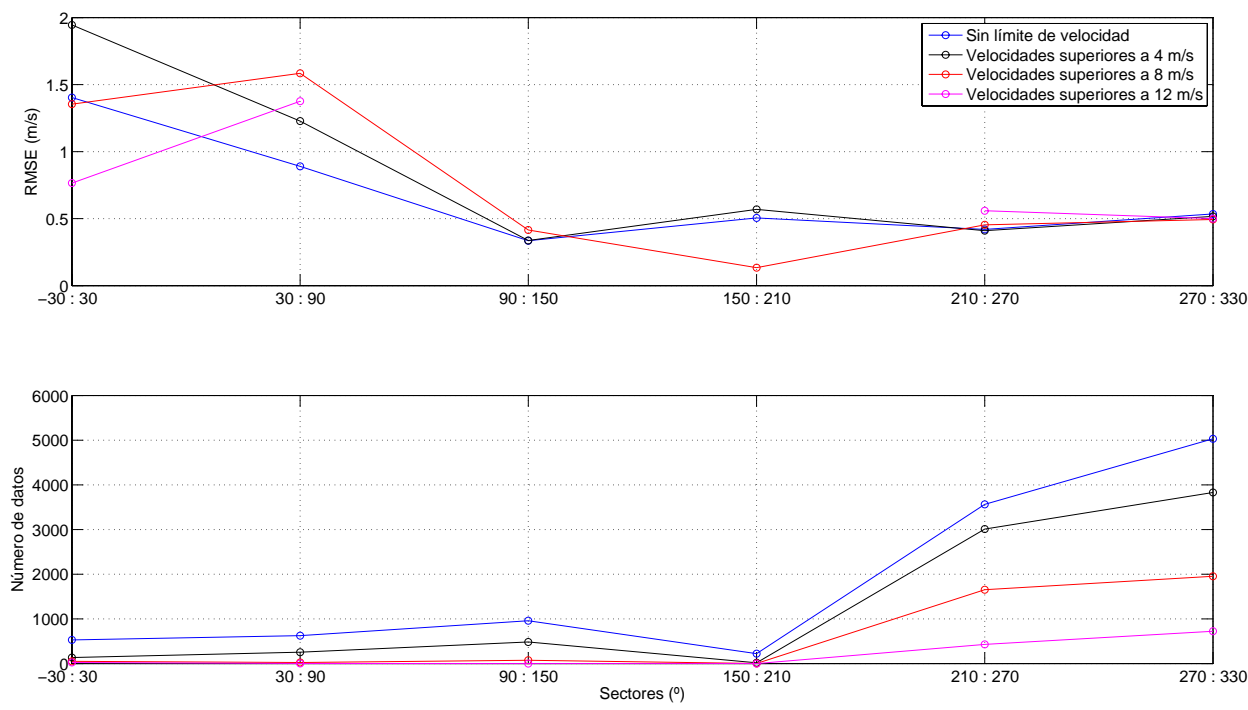

(b) Filtro de velocidades elevadas de viento.

Figura 3.36: RMSE calculado en función de las direcciones del viento en el parque eólico 3. 

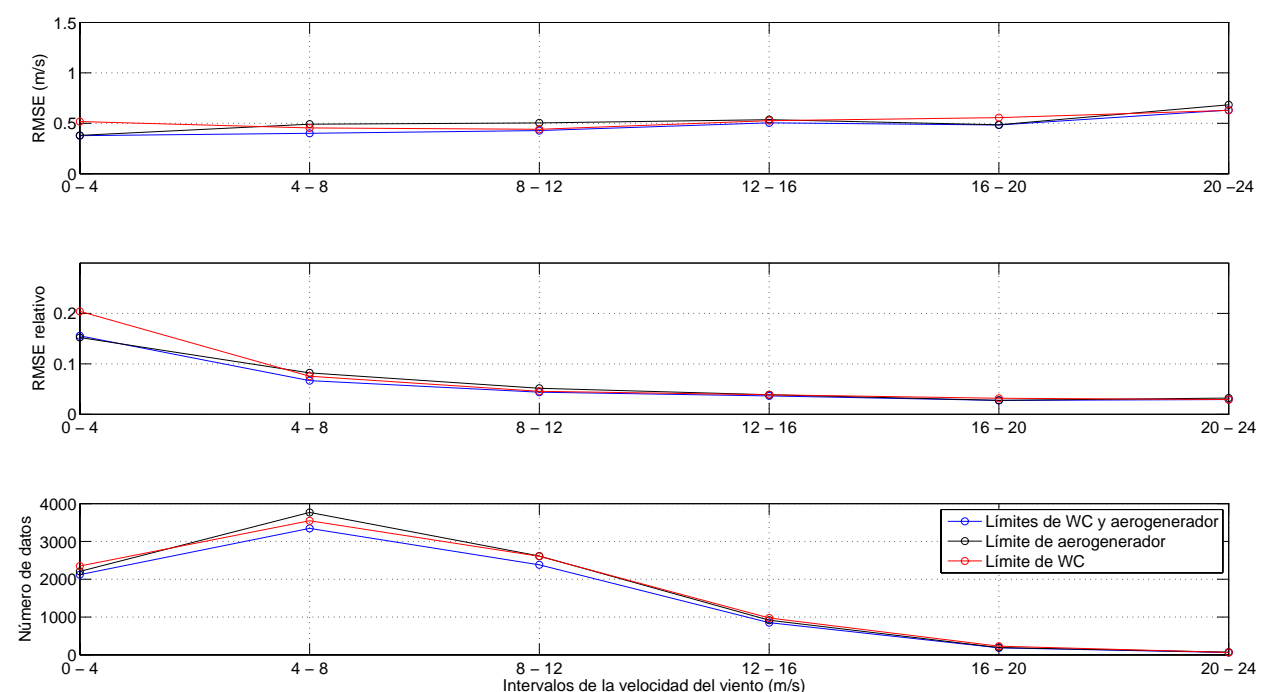

Figura 3.37: RMSE calculado en función de las velocidades del viento en el parque eólico 3, aplicando filtro de direcciones de viento.

Como puede verse en la figura 3.38 , las pendientes obtenidas en las correlaciones poseen valores próximos a 1 - lo que sería significativo de un ajuste perfecto entre equipos-, excepto para el sector 3, en el que la torre meteorológica podría estar haciendo sombra al WindCube y el número de muestras es más reducido que en el resto de sectores, tal y como se comentó anteriormente.

Con el mismo interés, la figura 3.39, muestra los valores medidos por el anemómetro de góndola del aerogenerador del parque eólico 3 en el eje de abscisas, frente a los registrados por el LiDAR en los mismos instantes en el eje de ordenadas (sin la información contenida en los sectores 1 y 2, como se comentó anteriormente). Debido a la proximidad con el valor 1 de la pendiente, que indicaría que se trata de una recta de $45^{\circ}$ de inclinación, y a la escasa dispersión de los datos, se puede afirmar que existe una buena correlación en la medida de la velocidad del viento entre los equipos.

Hay que señalar que los resultados obtenidos en relación con la correlación de las velocidades del viento apuntan hacia la misma dirección que los presentados en Foussekis et al. (2007); Foussekis (2009), donde se evaluó el comportamiento de diferentes equipos LiDAR sobre terreno complejo.

También es importante tener en cuenta que, aunque los resultados obtenidos sobre la correlación de la velocidad del viento pueden considerar que el equipo LiDAR se aproxima con gran precisión a los tradicionales, existe una diferencia considerable con los experimentos realizados por Smith et al. (2006); Antoniou et al. (2006, 2007a); Gottschall y Courtney (2010); Al- 

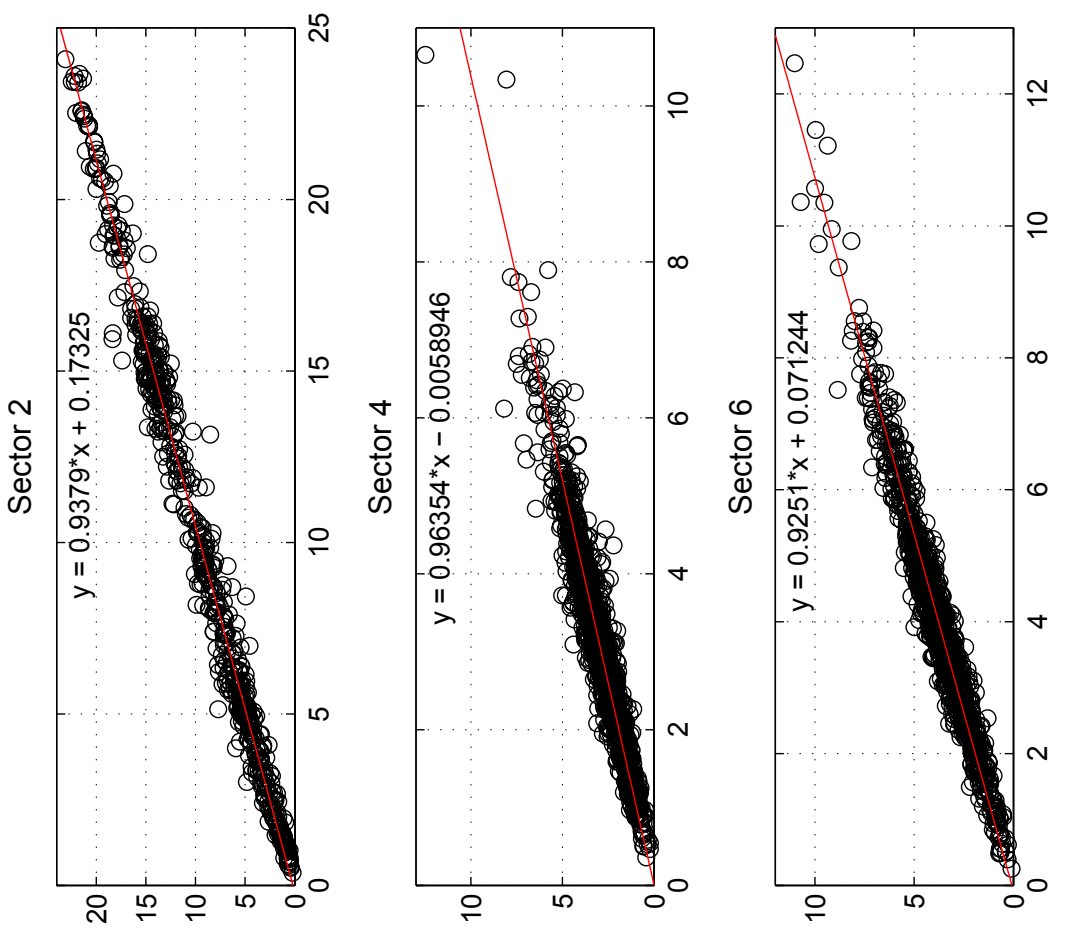

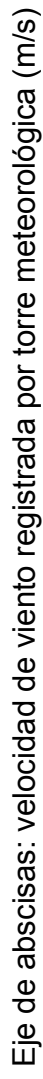
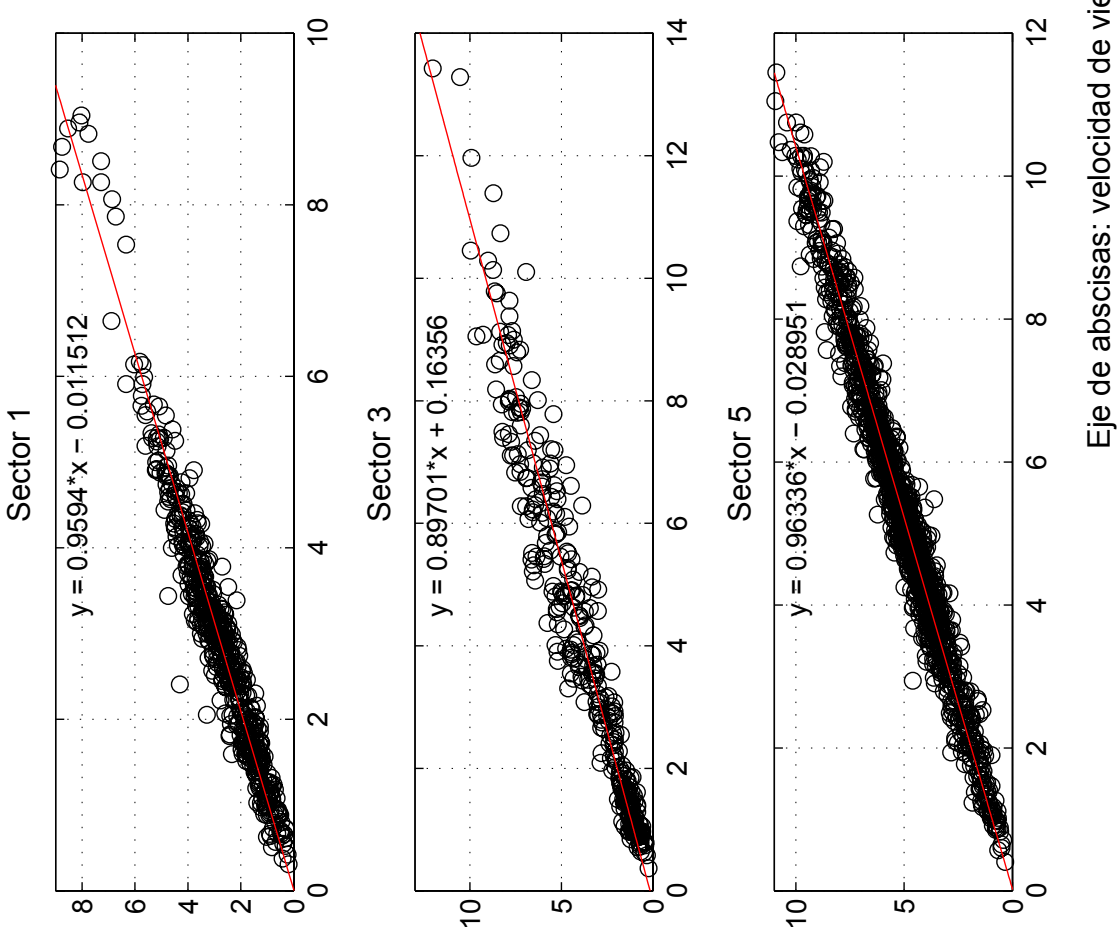

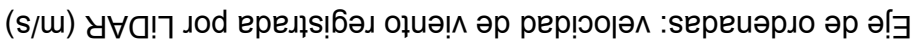

Figura 3.38: Correlación de la velocidad del viento en tunción de direcciones del viento en el parque eólico 1. 


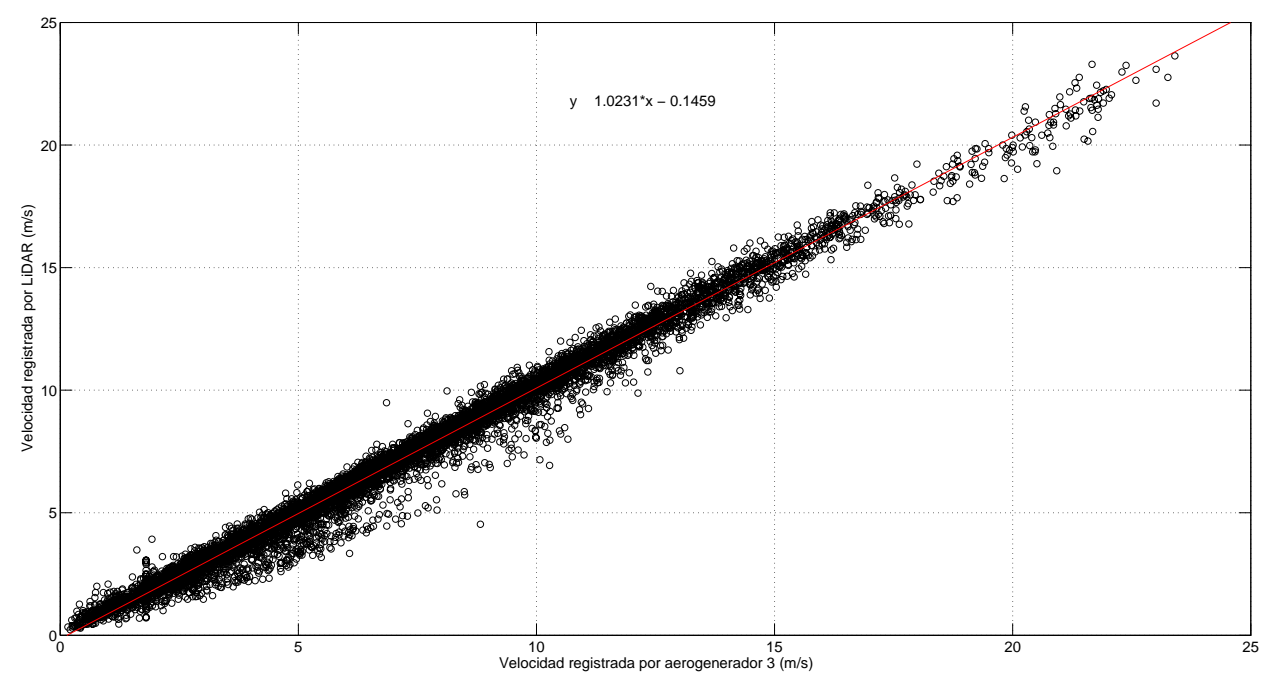

Figura 3.39: Correlación de la velocidad del viento filtrada en el parque eólico 3.

bers et al. (2012). Sin embargo, esta discrepancia es principalmente debida a que los experimentos realizados por estos autores eran todos sobre terreno llano y los llevados a cabo en la presente Tesis Doctoral implican terrenos de orografía especialmente compleja.

\subsubsection{Efecto sobre la desviación estándar}

Igual que se realizó con el registro de velocidades del viento, también se ha implementado el ajuste por mínimos cuadrados entre la desviación estándar de las velocidades registradas por el anemómetro Thies First Class y el WindCube instalados en el parque eólico 1, figura 3.40 .

Debido al elevado valor de la pendiente de la recta de ajuste de la figura 3.40 se puede afirmar que existe un elevado grado de correlación entre las desviaciones estándar de las velocidades registradas por ambos equipos.

En otras referencias bibliográficas, como Antoniou et al. (2007a); Foussekis et al. (2007), el resultado conseguido en la correlación de las desviaciones estándar de las velocidades registradas por ambos equipos resulta significativamente más desfavorable que el que se ha dibujado en la figura 3.40 . Esta circunstancia, puede estar relacionada con que los equipos utilizados en esas referencias emplearon diferentes frecuencias de muestreo, mientras que los equipos utilizados en la presente Tesis Doctoral poseen idéntica frecuencia de muestreo. 


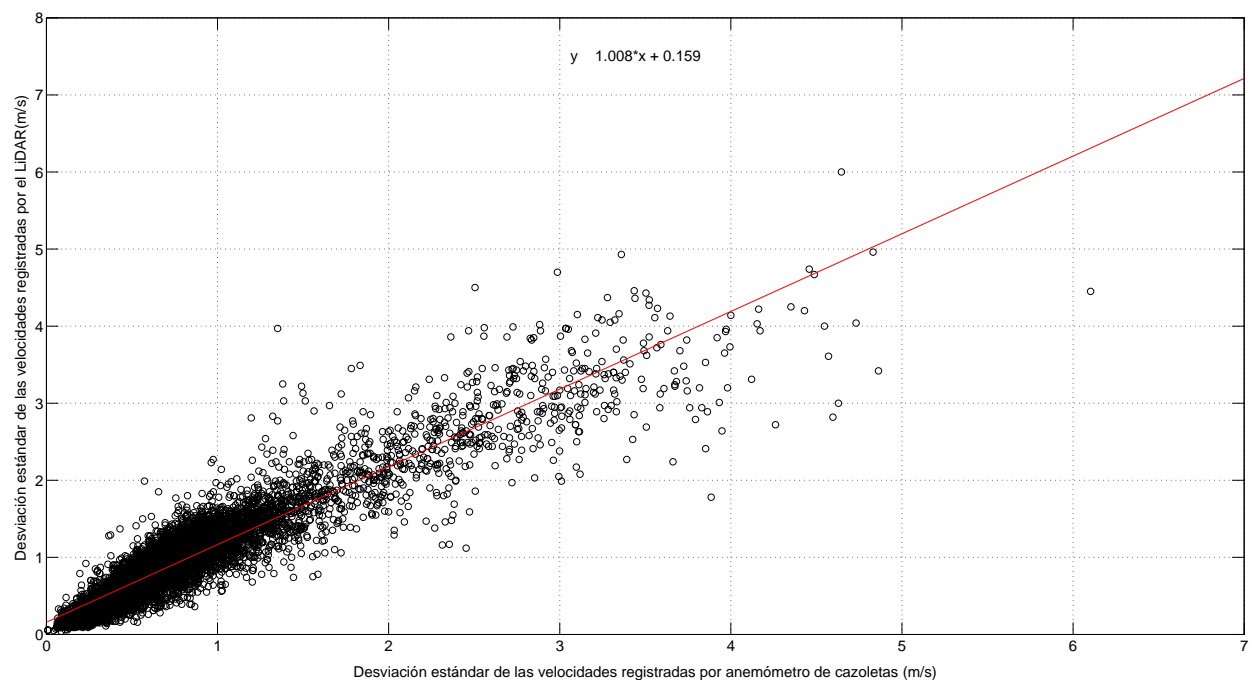

Figura 3.40: Correlación de la desviación estándar de la velocidad del viento en el parque eólico 1.

\subsubsection{Efecto sobre dirección del viento}

Para finalizar el análisis sobre la comparativa entre las mediciones llevadas a cabo por sistemas de anemometría láser y sistemas convencionales, en el presente apartado se va a estudiar la relación entre la dirección del viento medida por las diferentes tecnologías en los parques eólicos 1 y 2 .

Si directamente se intentase ajustar una función lineal por mínimos cuadrados a los valores de dirección del viento registrados entre diferentes equipos, se obtendría una elevada dispersión en las zonas cercanas a $360^{\circ}$. Esto podría ocasionar unos aparentes errores que no son reales, pues se deben a pequeñas diferencias en torno a $0^{\circ}$, ya que la medida que realice un equipo cercana a $0^{\circ}$ realmente posee reducido error con respecto a la que otro equipo registre cercano a $360^{\circ}$.

Para corregir este efecto se han tenido en cuenta dos filtros:

- Si la diferencia entre cada medida realizada por el WindCube y la torre meteorológica es superior a $180^{\circ}$, entonces se añaden $360^{\circ}$ a la menor medida. De esta manera, el problema señalado en el párrafo anterior queda solucionado.

- Se han filtrado todas las direcciones del viento para velocidades mayores de $4 \mathrm{~m} / \mathrm{s}$. Con este filtro se consigue evitar las grandes variaciones en la dirección del viento que ocurren a baja velocidad

Mediante la implementación de estos dos filtros, se ha ajustado una línea recta en el parque eólico 1 , figura 3.41 de ecuación $y=0,9793 \cdot x-9,8037$. A 


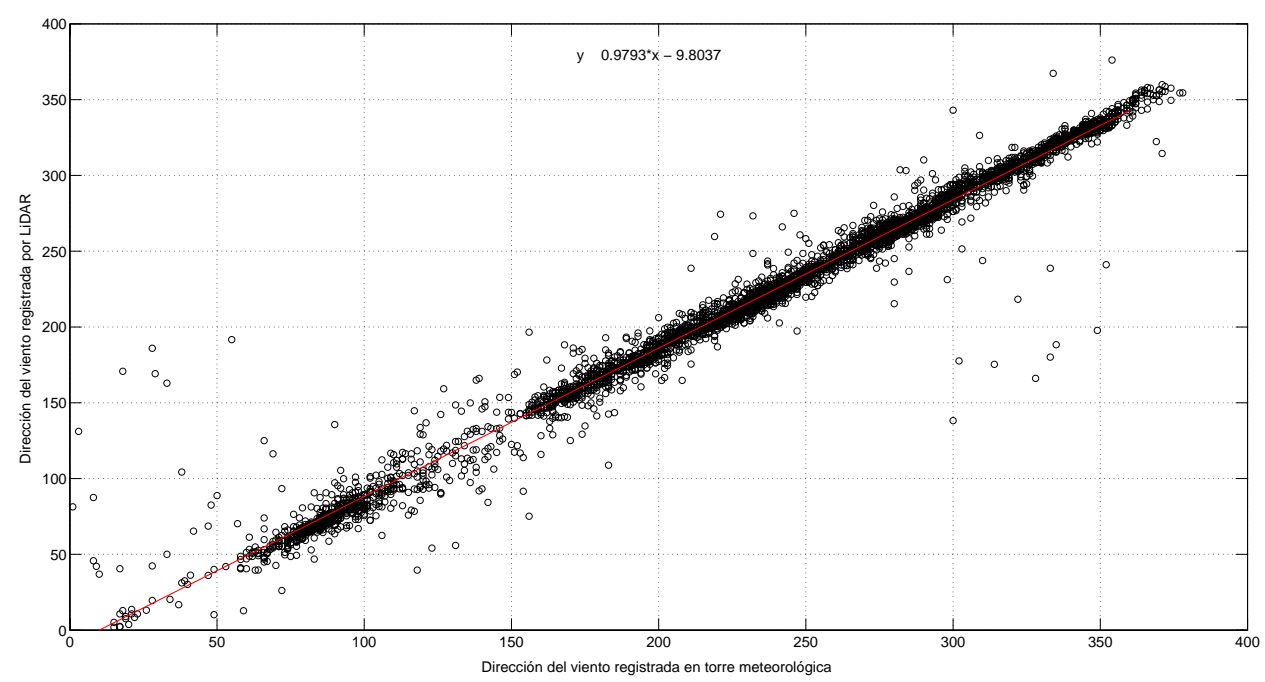

Figura 3.41: Correlación de la dirección del viento en el parque eólico 1.

raíz de esta figura, se puede observar que existe una elevada correlación en la medida de la dirección del viento entre ambos equipos, aunque se observa un error de offset significativo. Este error está directamente relacionado con lo que se comentó en el apartado 3.3.3.1. es decir, es debido a un error humano causado por una errónea alineación del equipo sobre el terreno.

Con el objeto de comprobar el resultado anterior, también se ha realizado este ajuste en el parque eólico 2. En este caso se obtiene un ajuste prácticamente perfecto de la anemoveleta Young y el LiDAR, con unas pendientes de ajuste de 1.0011 (45 m de altura) y 0.9942 (69 m de altura). También, igual que ocurrió en el parque eólico 1, ha aparecido un offset, aunque de valor inferior al que se obtuvo en dicho parque.

A raíz de los resultados que se han mostrado, se puede afirmar que existe una buena correlación en la medida de la dirección del viento entre ambos equipos, aunque el valor de la ordenada en el origen debe ser tenido en consideración.

Por lo tanto, una vez que se ha comprobado que existe un elevado grado de correlación entre las medidas que realiza un equipo LiDAR y las que realizan los sistemas tradicionales de medición del recurso, se está en condiciones de abordar el último tema que será tratado en el presente capítulo.

\subsubsection{Influencia del gradiente de viento en la generación de energía eólica}

Para concluir los resultados obtenidos en el presente capítulo, a continuación se analizará la influencia de los perfiles de viento así como de la 
velocidad equivalente en la generación de energía, es decir, en la curva de potencia de un aerogenerador. Para ello, se tendrán en cuenta los datos registrados en el parque eólico 3, así como las conclusiones derivadas de las publicaciones realizadas en Honrubia et al. $(2010 \mathrm{~b} d, 2012)$.

En base a la explicación teórica introducida en el apartado 3.1.3, la figura 3.42 muestra la curva de potencia de los nueve aerogeneradores del parque eólico 3 registrada durante el período de medidas utilizando el anemómetro de cazoletas que se encuentra situado en la góndola de cada aerogenerador. Obviamente, esta primera figura no muestra la forma normalizada por el método de los bines impuesta por la Norma IEC 61400-12-1.

De igual manera, atendiendo a las indicaciones de dicha Norma, para caracterizar la curva de potencia de un aerogenerador se debe tener en cuenta que la medida de la velocidad del viento debe realizarse a una distancia del aerogenerador entre 2 y 4 diámetros de rotor; siendo 2.5 diámetros de rotor el valor recomendado. Dado que en el parque eólico 3 se utilizará la velocidad de viento registrada por el LiDAR (ya que es el único equipo del parque capaz de obtener los parámetros eólicos a varias alturas) y, teniendo en cuenta la distribución de los aerogeneradores sobre el terreno (ver figura 3.19) y que éstos tienen un diámetro de rotor de $90 \mathrm{~m}$, el aerogenerador que cumple los requisitos señalados anteriormente y en el que en su área barrida se incluyen mayor número de medidas realizadas por el anemómetro láser es el número 2. Por lo tanto, el aerogenerador 2 del parque eólico 3 ha sido seleccionado para realizar el análisis de la curva de potencia.

Igualmente, la citada Norma señala que deben ser eliminados del estudio los sectores de viento en los que puedan existir obstáculos que dificulten la correcta evaluación de la curva de potencia. En este sentido, para analizar en detalle los sectores que deben ser eliminados en este parque eólico, en la figura 3.43 se ha realizado un estudio del RMSE entre la velocidad del viento medida por el LiDAR en su tercera altura de registro y el anemómetro de la góndola del aerogenerador 2 en función de la dirección del viento registrada por el LiDAR en su tercera altura de registro. La primera idea que se puede deducir de la figura 3.43 es que su forma es similar a la mostrada en la figura 3.36, con la salvedad de que el valor del error es ligeramente superior en la figura 3.43 debido principalmente a la mayor distancia entre equipos. Además, en vista de la figura 3.43, es necesario eliminar del cálculo de la curva de potencia los sectores 1,2 y 4 ; ya que en dichos sectores se eleva el RMSE. Un aspecto positivo a tener en cuenta es que eliminar dichos sectores implica eliminar poca información, ya que, como se observa en la figura, la mayoría de los datos se encuentran en los sectores 5 y 6 .

En base a las consideraciones anteriores y a lo comentado en el apartado 3.1.3 en relación con el cálculo de la velocidad equivalente, en un primer análisis se ha dividido el área del rotor del aerogenerador 2 en seis secciones, con el interés de emplear las seis primeras alturas registradas por el LiDAR. 

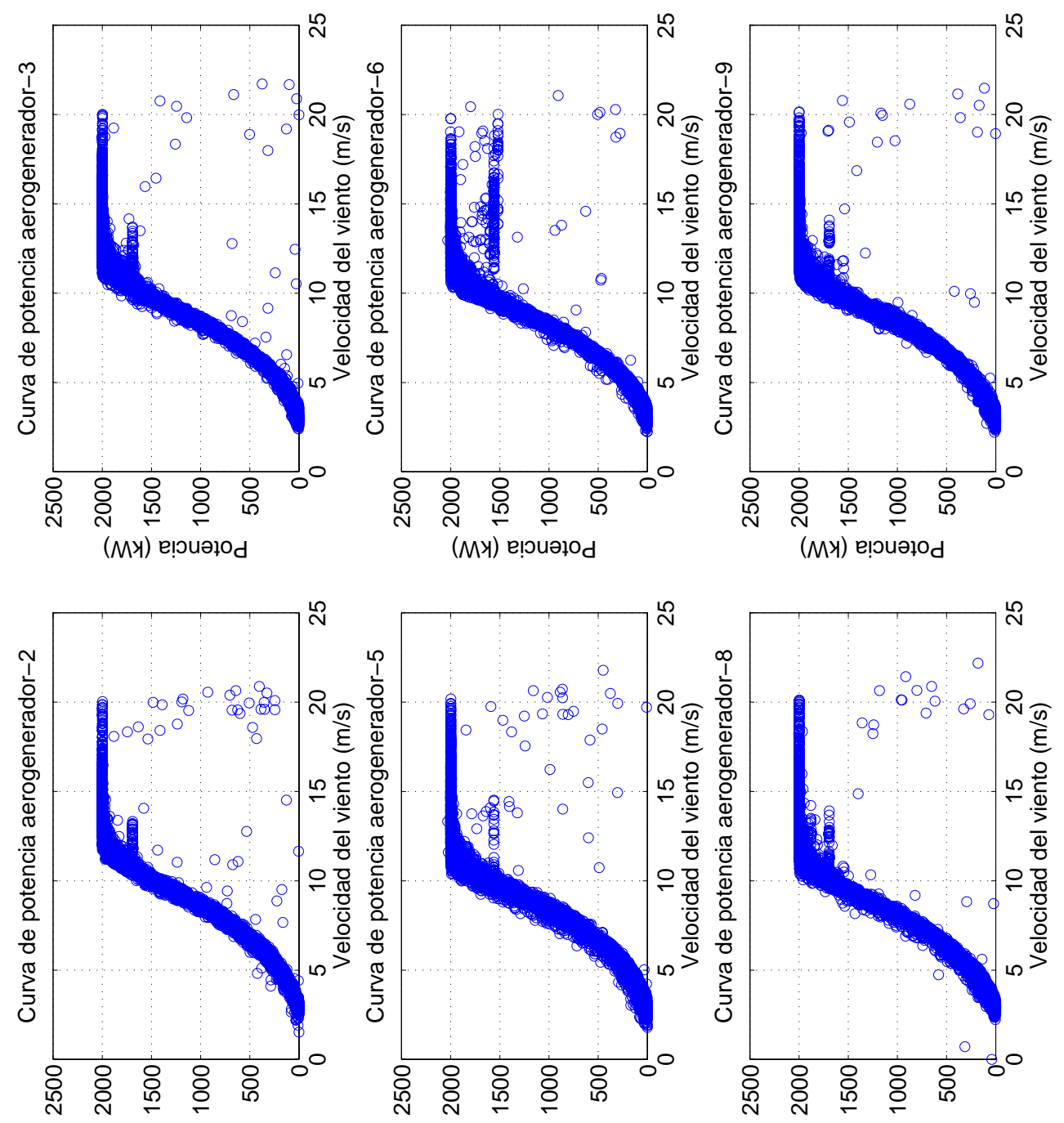

(MY) e!̣uәjod

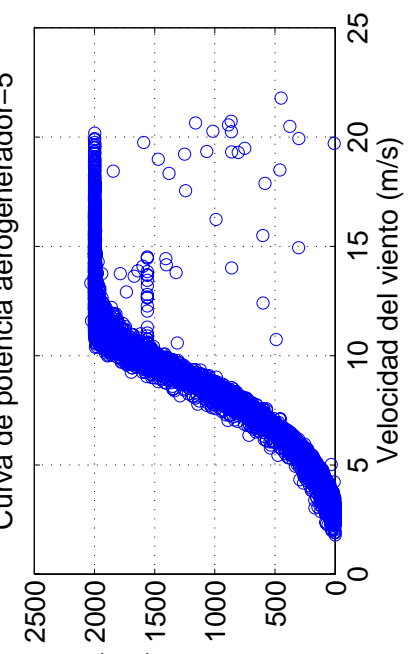

(My) e!̣uәрод
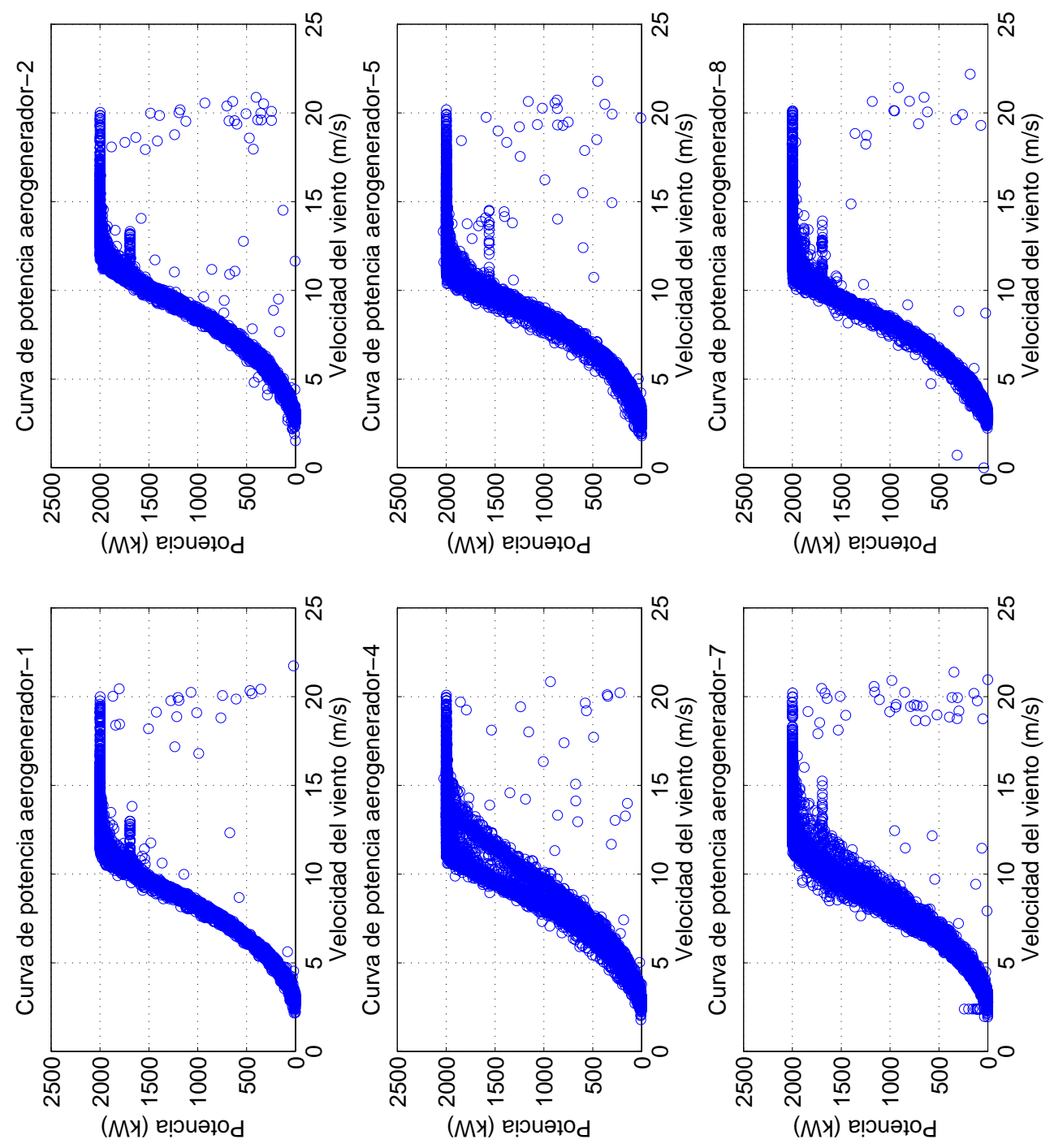

Figura 3.42: Curvas de potencia de los nueve aerogeneradores del parque eólico 3. 

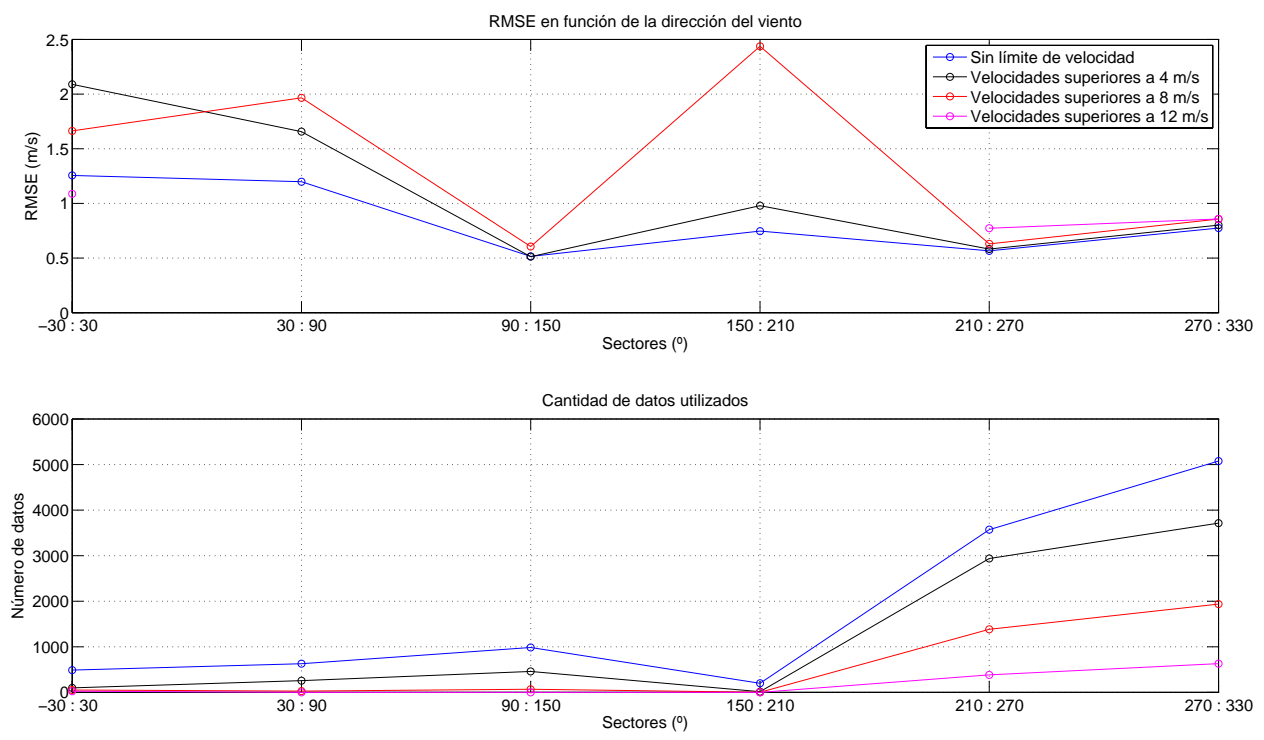

Figura 3.43: RMSE entre LiDAR y aerogenerador 2 del parque eólico 3 en función de las direcciones del viento registradas por el WindCube con diferentes filtros de velocidades.

La figura 3.44 muestra las alturas de medición empleadas así como las áreas obtenidas. Como puede observarse, las áreas 2 a 6 tienen un valor muy similar, mientras que el área 1 es considerablemente superior a las demás dado que no existen velocidades de viento registradas por debajo de $40 \mathrm{~m}$. Se vuelve a señalar que el reparto de áreas se ha realizado de esta manera con el objetivo de emplear directamente las velocidades registradas por el equipo LiDAR en lugar de utilizar aproximaciones teóricas para la obtención de velocidades de viento en otras alturas.

Por lo tanto, teniendo en cuenta los requisitos impuestos anteriormente, la figura 3.45 muestra cuatro formas diferentes de obtener la curva de potencia del aerogenerador 2 con el método de los bins. La línea azul muestra la curva de potencia utilizando solamente su propio anemómetro de góndola. La roja representa la medida por el WindCube en su tercera altura de medición - ya que esta altura coincide con la de buje del aerogenerador 2 teniendo en cuenta la elevación sobre el terreno de ambos elementos-, y las negras las medidas en función de las velocidades equivalentes definidas en las ecuaciones (3.11) y 3.12 .

A raíz de la figura 3.45 se pueden obtener diversas conclusiones. Por un lado, se observa cómo la curva de potencia responde a la curva ideal de un aerogenerador de $2 \mathrm{MW}$ de potencia nominal, con sus zonas de funcionamiento claramente diferenciadas. Pero, haciendo zoom sobre la misma, se observan las características más importantes: en cuanto a la curva de potencia ob- 


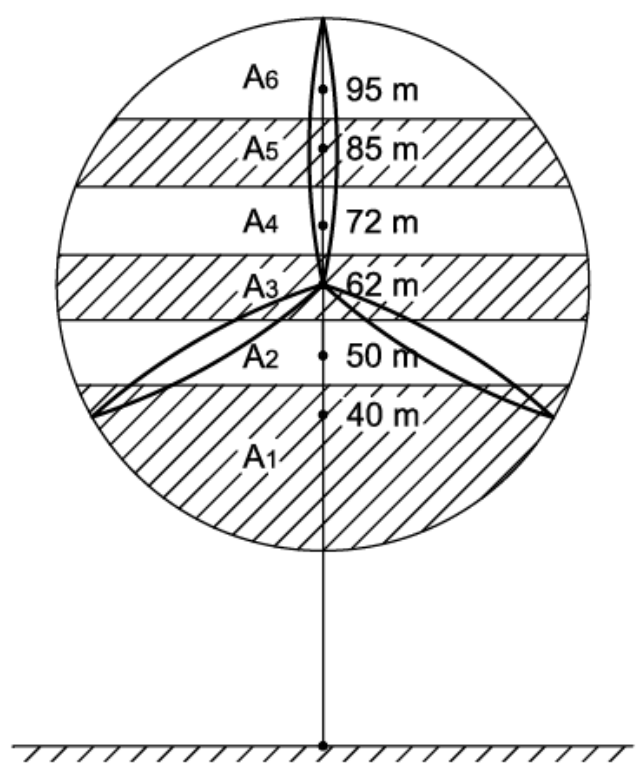

Figura 3.44: Área barrida del aerogenerador 2 dividida en 6 secciones.

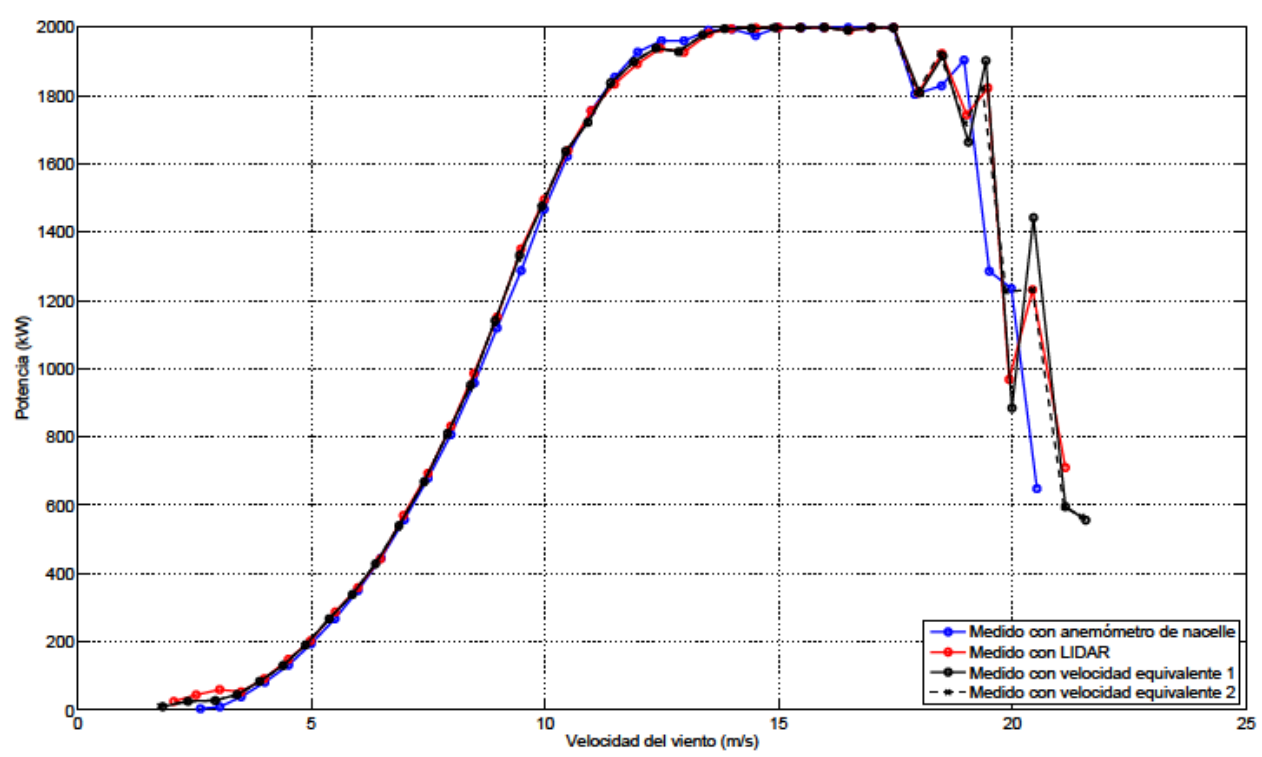

Figura 3.45: Curvas de potencia del aerogenerador 2. 
tenida mediante el empleo de cualquier velocidad de viento equivalente, se observa que el valor de la velocidad equivalente es ligeramente inferior al de la velocidad medida por LiDAR o por anemómetro de góndola. Este hecho se encuentra estrechamente relacionado con el reparto de áreas seleccionado mostrado en la figura 3.44. Debido a que el área 1 es sustancialmente superior al resto de áreas, el valor de la velocidad del viento registrado a $40 \mathrm{~m}$ tiene mayor peso que el resto de áreas.

Por lo tanto, para un cálculo de la velocidad equivalente en el que todas las velocidades tengan el mismo peso, es necesario que todas las áreas tengan el mismo valor. Con esta intención, a continuación se ha dividido el área del rotor en 6 secciones completamente idénticas. Lógicamente, como en esta ocasión las velocidades registradas por el equipo LiDAR no son coincidentes con el centro de cada sección, es necesario emplear la Ley Potencial para la obtención del perfil de viento en las alturas donde no se conoce, ecuación (3.4). Mediante el empleo de dicha ecuación, primero se ha calculado el exponente de cortadura de forma dinámica en cada instante de tiempo para dos velocidades y alturas conocidas y, una vez conocido este $\alpha$, se ha calculado la velocidad del viento en el punto exacto donde se precisa.

La figura 3.46 muestra el reparto de áreas teniendo en consideración este segundo supuesto de igualdad de áreas. En el eje de simetría vertical de la figura se han señalado las alturas en las que se debe de tener la medida del viento teniendo en cuenta el centro de gravedad de cada región. Como se observa, sólo en el caso de la sexta región coincide con una altura de medida real del sistema LiDAR; con lo que para el resto de regiones se tendrán que calcular las velocidades en función de (3.4). Bajo estas premisas, la figura 3.47 muestra la curva de potencia del aerogenerador 2 empleando el método de los bins de las cuatro diferentes maneras definidas anteriormente para la figura 3.45

Lógicamente, la única diferencia entre las figuras 3.45 y 3.47 se representa mediante el trazo de color negro, es decir, la curva de potencia obtenida con el empleo de las velocidades equivalentes. En la primera curva que se obtuvo, figura 3.45, existía un reparto de áreas que otorgaba mayor peso a las mediciones efectuadas por el equipo LiDAR a $40 \mathrm{~m}$ de altura; sin embargo, en la figura 3.47 se corrigió este hecho asumiendo áreas iguales y velocidades obtenidas mediante la ecuación (3.4). Por lo tanto, en la figura 3.47 se observa que la curva de potencia obtenida con las velocidades equivalentes es prácticamente idéntica a la generada mediante el equipo LiDAR hasta velocidades cercanas a la de corte del aerogenerador. Concretamente, para altas velocidades de viento cercanas a las de corte, una mejora apreciable se observa; ya que se produce un descenso suave en la potencia generada (ver intervalo de $17 \mathrm{~m} / \mathrm{s}$ a $19 \mathrm{~m} / \mathrm{s}$ en la figura 3.47) en lugar de producirse cambios bruscos en la potencia (ver mismo intervalo de velocidades de viento en la figura 3.45 . 


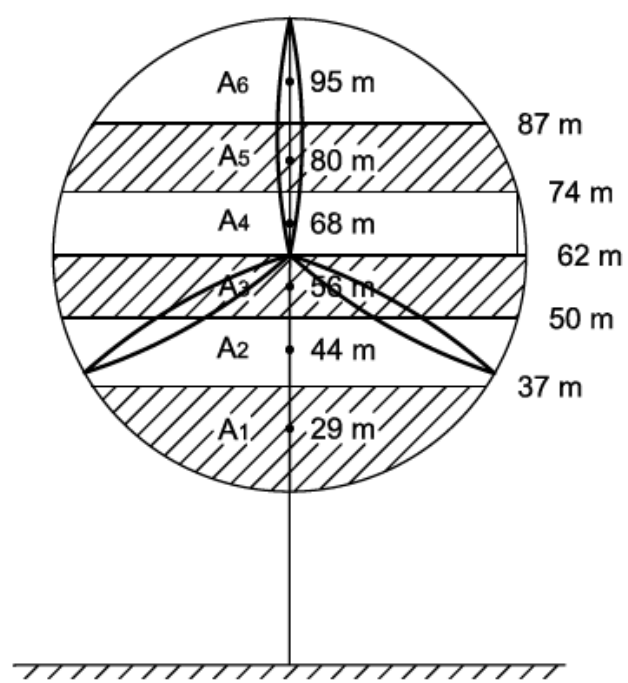

Figura 3.46: Área barrida del aerogenerador 2 dividida en 6 secciones idénticas.

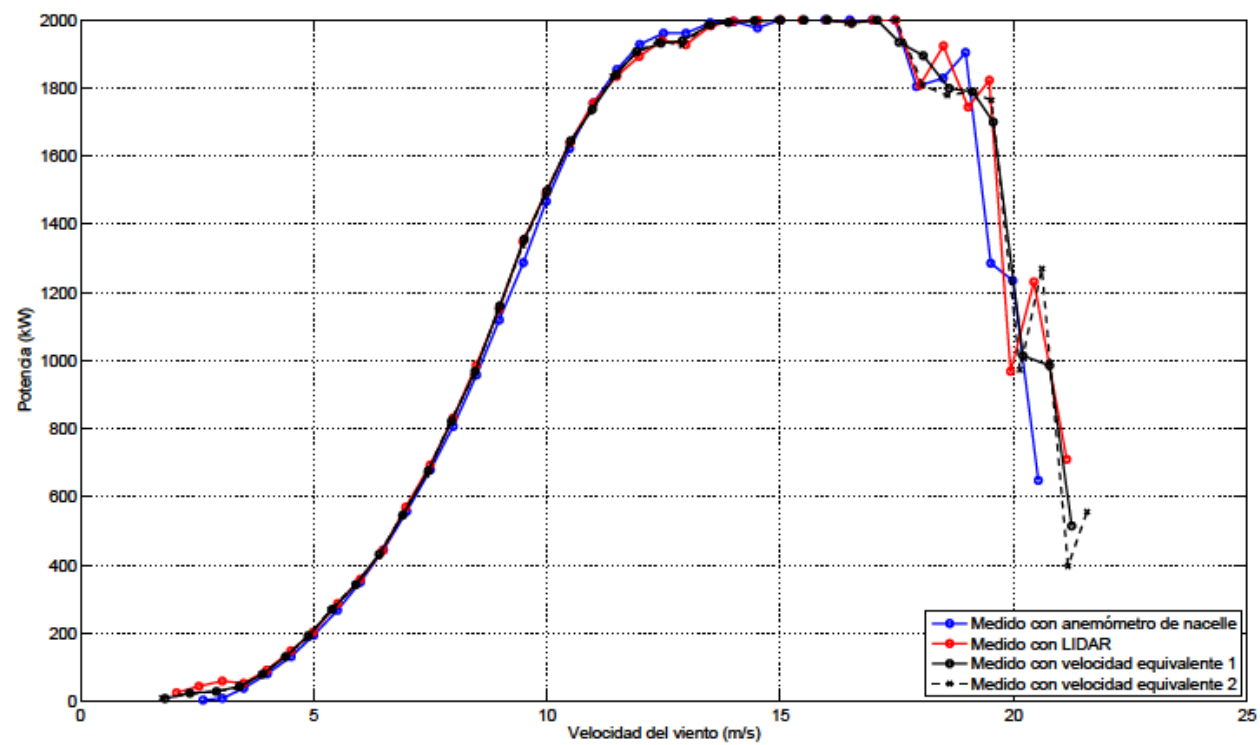

Figura 3.47: Curvas de potencia del aerogenerador 2. Velocidades equivalentes obtenidas mediante criterio de igualdad de áreas. 


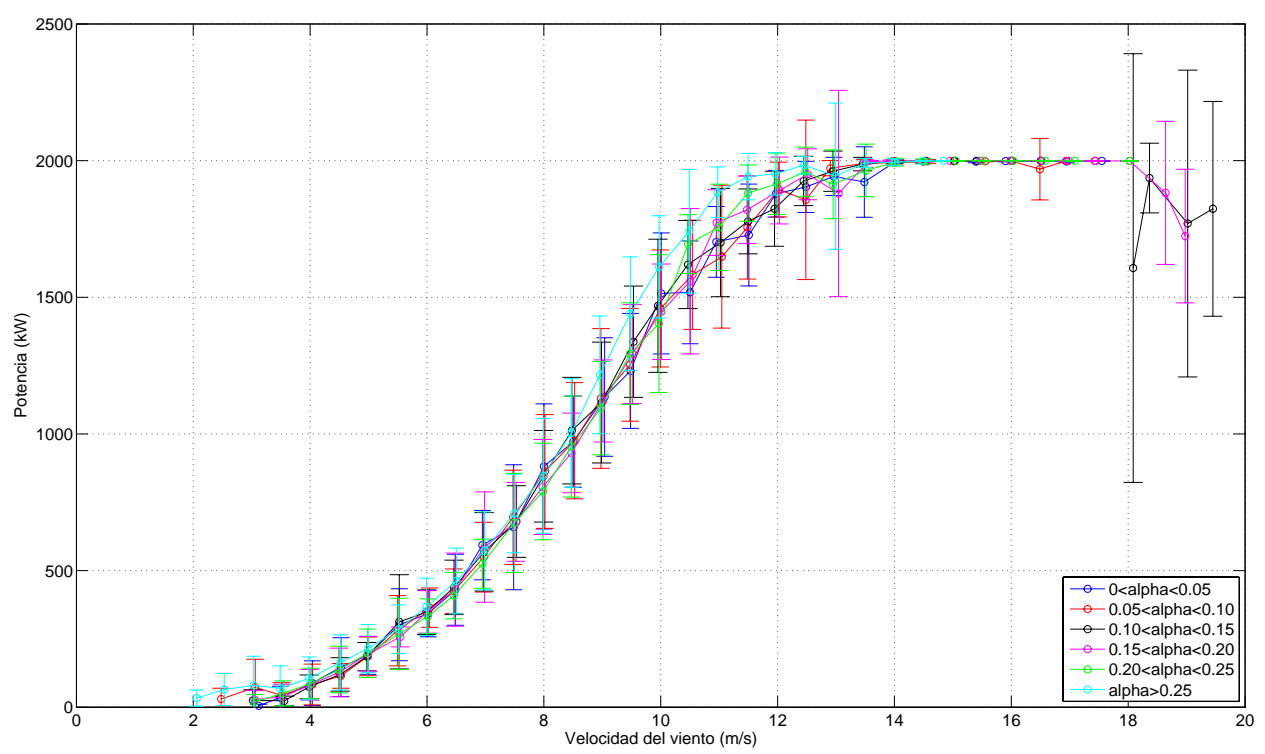

Figura 3.48: Curva de potencia del aerogenerador 2 en función del coeficiente de cortadura, $\alpha$.

En vista de los resultados anteriores, se puede afirmar que el empleo de velocidades de viento equivalentes que tienen en cuenta todo el perfil de viento que existe delante del aerogenerador implica la corrección de la curva de potencia de la turbina para velocidades de viento próximas a las de corte. Además, se ha demostrado la necesidad de que el rotor se divida en regiones idénticas. Es importante tener en cuenta que el estudio realizado está basado sobre aerogeneradores de $78 \mathrm{~m}$ de altura; mientras que actualmente existen aerogeneradores con alturas de buje considerablemente superiores.

Una vez vista la importancia de tener en cuenta no solo la altura de buje para la caracterización de la curva de potencia se va a analizar la influencia de los perfiles de viento en la energía generada.

Por ello, en la figura 3.48 se presenta la curva de potencia del aerogenerador 2 medida con el equipo LiDAR en función de las condiciones atmosféricas, agrupadas éstas según el coeficiente de cortadura (éste se ha calculado de acuerdo a las velocidades registradas por el WindCube entre 62 y $72 \mathrm{~m}$ de altura). Además, se ha dibujado la desviación estándar que existe en cada bin.

Observando la figura 3.48 , queda probado el alto grado de dependencia entre la potencia producida por el aerogenerador y el gradiente de viento incidente sobre el mismo. Se observa que un mayor coeficiente de cortadura (idéntico a decir "elevado gradiente de viento" o "perfil de viento no plano") se traduce en una respuesta no esperada en la potencia generada para velocidades de viento superiores a $8 \mathrm{~m} / \mathrm{s}$. Concretamente, se observa que cuando 
se tienen medios y altos coeficientes de cortadura, la potencia generada por el aerogenerador es superior que la que se produce con reducidos coeficientes de cortadura.

Dicho de otra manera, atendiendo a los perfiles de viento medios horarios mostrados en las figuras 3.26 y 3.27 durante las horas del día, la potencia generada es inferior que la que se produce durante las horas nocturnas para una misma velocidad de buje, dado que durante las horas nocturnas los perfiles de viento en el emplazamiento analizado se caracterizan por una alta estabilidad. Por lo tanto, queda justificado que para una correcta evaluación de la curva de potencia de un aerogenerador, el exponente de cortadura es un parámetro que debe ser considerado. 


\subsection{Conclusiones}

En el presente capítulo de la Tesis Doctoral se ha expuesto el tema de los sistemas remotos para la medición del recurso eólico, en concreto, la tecnología LiDAR. Se ha comprobado su validez con respecto a los métodos tradicionales en base a diferentes campañas de medidas llevadas a cabo en tres parques eólicos distribuidos por diversas regiones de España. También, ha servido para obtener conclusiones relacionadas con el gradiente de viento vertical. Finalmente, se ha evaluado la influencia del perfil de viento medido con este tipo de equipos sobre la generación de energía eólica. Todo ello ha sido llevado a cabo dentro del ámbito de la reforma de la Norma IEC 6140012-1, relativa a la caracterización de la curva de potencia de aerogeneradores. La investigación ha sido realizada en terrenos de orografía compleja que, aún tratándose del tipo de terreno habitual en los parques eólicos que se pueden encontrar en España - al contrario que el caso de otros países como Dinamarca, por ejemplo-, el número de estudios encontrados sobre este tipo de terreno es reducido.

Un primer aspecto a señalar es el tema de la disponibilidad de este tipo de equipamiento. Se ha podido comprobar cómo se reduce la disponibilidad del equipo LiDAR WindCube a medida que se aumenta la altura de monitorización. Por lo tanto, no se está en condiciones de afirmar que sea recomendable el empleo de este tipo de tecnologías de manera aislada, sino en combinación con los sistemas tradicionales de evaluación del recurso eólico.

En relación con las mediciones del recurso eólico efectuadas con el equipo LiDAR, la primera conclusión ha sido que el gradiente de viento, así como los diferentes perfiles de viento registrados, varían cada hora de manera relevante. Incluso, para ciertas horas, se desvía considerablemente de las ecuaciones teóricas que se emplean actualmente en el mundo de la energía eólica. De hecho, en algunas ocasiones se han medido perfiles de viento en los que la velocidad comenzaba a disminuir por encima de una determinada cota sobre el terreno, contrariamente a lo que indican las aproximaciones teóricas. También, se han mostrado perfiles de viento con una forma muy diferente registrados en un intervalo de 12 horas.

Respecto a la evaluación del comportamiento del sistema LiDAR con respecto a sistemas convencionales, se ha efectuado un extenso análisis. Como se ha comprobado, el equipo WindCube ha mostrado alto grado de correlación frente al anemómetro de cazoletas convencional. Exceptuando los sectores de dirección del viento en que la torre meteorológica podía estar interfiriendo las medidas del equipo LiDAR, los resultados sobre la velocidad del viento registrada son altamente satisfactorios. La correlación obtenida no es tan perfecta como algunos estudios encontrados en la bibliografía, debido a que éstos han sido llevados a cabo sobre terreno llano, donde este tipo de equipos presentan el mejor comportamiento debido a su principio de operación. Además, en los 
tres parques eólicos donde se ha instalado el LiDAR se ha señalado que la velocidad del viento influye positivamente en la correlación, es decir, que a velocidades de viento mayores, el error cometido por el anemómetro láser es más reducido. Esta situación se encuentra relacionada con la pérdida de precisión que tiene el anemómetro de cazoletas a bajas velocidades debido a la inercia que acumula en rotación. También, se ha señalado que la diferencia aparecida en las mediciones entre equipos a bajas velocidades tienen un carácter menos relevante en el ámbito de la energía eólica, dado que es necesario superar un valor de velocidad de viento para que el aerogenerador comience a generar energía. Otro factor a tener en cuenta, es la altura a la que el equipo efectúa la medida de la velocidad del viento; ya que se ha comprobado que el error cometido aumenta con al altura.

En la misma línea, después de un filtrado específico, la dirección del viento registrada por el LiDAR y por veletas convencionales muestra una excelente correlación.

También, se ha comprobado que el efecto de sombra puede aparecer entre el equipo LiDAR y un obstáculo cercano, como es el caso de una torre meteorológica. De esta manera, resulta necesario un filtrado de información eliminando los obstáculos que puedan afectar a las medidas efectuadas por el equipo remoto. Concretamente, se ha conseguido una reducción del error del $20 \%$ aplicando este tipo de filtro.

Desde el punto de vista de la generación eólica, se ha verificado que el empleo de velocidades de viento equivalentes que tienen en cuenta todo el gradiente de viento situado delante del aerogenerador implica una mejora en la curva de potencia del mismo, dado que se suaviza su comportamiento para velocidades de viento próximas a las de corte. Además, se ha demostrado la necesidad de que el rotor se divida en áreas idénticas para la obtención de la velocidad equivalente.

En esta misma línea, también se ha tenido en cuenta el comportamiento del aerogenerador bajo diferentes condiciones del gradiente de viento. Los resultados han mostrado que cuando el viento que se encontraba delante del aerogenerador se caracterizaba por un elevado valor del coeficiente de cortadura o, dicho de otra manera, el perfil de viento que existía en ese momento era muy acusado, la energía producida por el aerogenerador era superior que la que se obtenía cuando se registraban perfiles de viento planos. Consecuentemente, en función del tipo de perfil de viento medio que se ha registrado en el parque eólico analizado, durante las horas del día la potencia generada es inferior que la que se produce durante las horas nocturnas para una misma velocidad del viento a la altura del buje, dado que durante las horas nocturnas los perfiles de viento en dicho parque se caracterizan por una alta estabilidad.

Con todo lo anterior, se está en condiciones de considerar que una reducción de la incertidumbre en la caracterización de la curva de potencia de 
aerogeneradores parece ser posible si la medida de la velocidad del viento se realiza en toda el área barrida del rotor y no se limita solamente a la medida de la velocidad del viento a la altura de buje. En esta línea, es importante tener en cuenta la tendencia que lleva existiendo desde hace unos años en el sector eólico, orientada hacia el progresivo aumento del tamaño de los aerogeneradores.

Por lo tanto, en este ámbito cabe mencionar que la nueva versión que se está realizando de la Norma sobre caracterización de curva de potencia tendrá en cuenta el empleo de estos de sistemas remotos así como la influencia del gradiente de viento en la producción eólica.

Por último, tal y como se ha ido comentando en el presente capítulo, la investigación que se ha llevado a cabo ha culminado con la publicación en varios congresos internacionales relacionados con el sector eólico, Honrubia et al. (2010a b c d, 2011), así como en una revista nacional de ingeniería, Honrubia Escribano et al. (2012a), y en un capítulo de un libro, Honrubia et al. (2012). 



\title{
Modelos simplificados de aerogeneradores
}

\begin{abstract}
Resumen: En este capítulo de la Tesis Doctoral se presenta el trabajo relacionado con los modelos simplificados de aerogeneradores. En una primera etapa introductoria se muestran las directrices que actualmente están siendo marcadas por el desarrollo de la nueva Norma IEC 61400-27. Posteriormente se describe la campaña de medidas realizada que se ha empleado para, posteriormente, parametrizar determinados modelos del aerogenerador. Finalmente, se muestran las conclusiones obtenidas.
\end{abstract}

\subsection{Introducción}

El progresivo aumento de la potencia eólica instalada en los sistemas eléctricos, representado en el apartado 1.1 de la presente Tesis Doctoral, implica que los operadores de dichos sistemas necesiten modelos dinámicos de generadores eólicos para poder realizar estudios de estabilidad que permitan evaluar el impacto de la integración de este tipo de energía en los sistemas eléctricos de potencia, Behnke et al. (2007); Fortmann (2010); Fortmann et al. (2010); Sørensen et al. (2011).

Concretamente, los operadores de los sistemas eléctricos realizan simulaciones con el objetivo de estimar el impacto de diferentes escenarios sobre la capacidad de dichos sistemas para soportar tales eventos, NERC (2010). Cuando una perturbación es simulada en un software de simulación de sistemas eléctricos y el resultado conseguido es una inadecuada respuesta de la red, los operadores deben diseñar diferentes estrategias para que el sistema 
responda de manera estable en el escenario ensayado. Para asegurar una correcta solución del problema, así como una mínima inversión en equipos, se requieren modelos de simulación que representen el tipo de generadores que se encuentran distribuidos por las redes eléctricas, Lin et al. (2009).

En este ámbito, se puede señalar que los modelos recientemente desarrollados por los diferentes fabricantes de aerogeneradores consiguen estimar el comportamiento eléctrico y mecánico de sus máquinas con un elevado nivel de precisión. De hecho, más de 25 modelos específicos de diversos fabricantes se encuentran disponibles en el software PTI PSS ${ }^{\circledR}$ E, bajo la firma Siemens, Keung et al. (2009); Asmine et al. (2011). Sin embargo, este grado de detalle no resulta idóneo para estudios de estabilidad de grandes sistemas de potencia debido a varios motivos. Por un lado, estos modelos detallados requieren una elevada cantidad de información de entrada para representar cada tipo de aerogenerador, Price y Sanchez-Gasca (2006). Además, debido al elevado número de parámetros que emplean, el tiempo de simulación así como el nivel de complejidad de este tipo de modelos resultan incrementados de manera considerable, Fortmann et al. (2010). Más aún, estos modelos normalmente se encuentran sujetos a contratos de confidencialidad, Ellis et al. (2011a); Hiskens (2012), que son válidos sólo para un tipo de aerogenerador específico, durante un período de tiempo determinado, NERC (2010). De esta manera, el trabajo que debe desarrollar el operador del sistema resulta complejo y con elevado coste computacional.

Teniendo en cuenta las anteriores consideraciones, la "International Electrotechnical Commission (IEC)" se encuentra actualmente desarrollando una nueva Norma Internacional, IEC 61400-27, dedicada a la definición de modelos genéricos — también conocidos como simplificados o estándar-, tanto de aerogeneradores (Parte 1 de la Norma), como de parques eólicos (Parte 2). Los modelos están diseñados para representar generación de energía eólica en estudios de estabilidad de tensión de los sistemas eléctricos en el corto plazo bajo grandes perturbaciones (se pueden consultar las referencias Kundur et al. (2004); Comech Moreno (2007) para conocer en detalle la clasificación de estabilidad de los sistemas eléctricos). Por lo tanto, estos modelos son útiles para estudios dinámicos ante perturbaciones de red, tales como las señaladas en el apartado 2.1.1, es decir, ante huecos de tensión; así como pérdidas de unidades de generación o demanda.

Una de las características perseguidas por los modelos genéricos que se están desarrollando en la IEC bajo esta temática es su implementación bajo cualquier software de simulación, Behnke et al. (2007); NERC (2010); así como la sencilla adaptación a cualquier modelo de fabricante a través de la modificación de los parámetros del modelo, Keung et al. (2009); Fortmann (2010); Ellis et al. (2011a). Continuando con la misma línea, algunos autores señalan que, para la realización de estudios de estabilidad de sistemas eléctricos, se puede considerar que la velocidad del viento es constante en 
un intervalo comprendido entre 5 s y 30 s, Price y Sanchez-Gasca (2006); Behnke et al. (2007); Keung et al. (2009); Asmine et al. (2011); Ellis et al. $(2011 \mathrm{a}$ b). Por lo tanto, esta es una de las hipótesis asumidas por este tipo de modelos simplificados.

Debido al escenario en el que se encuentra inmersa la IEC 61400-27, presentado en las líneas anteriores, existen diversos sectores especialmente interesados en el desarrollo de la misma:

- Operadores de red: dado que son los responsables de la operación y planificación de los sistemas eléctricos de potencia.

- Propietarios de parques eólicos: en algunos casos los propietarios de los parques tienen la responsabilidad de entregar los modelos de sus parques a los operadores de red antes de la puesta en marcha del parque, Ravalli y Leung (2011); Asmine et al. (2011).

- Fabricantes de aerogeneradores: dado que éstos son los que comúnmente entregan los modelos al propietario.

- Desarrolladores de software relacionado con la simulación de sistemas de energía eléctrica.

- El sector de la investigación también constituye una parte interesada, debido a que la confidencialidad inherente de los modelos detallados de fabricantes dificulta el acceso a la información.

Con todo lo anterior, algunos grupos de investigación afirman que, hoy en día, los modelos genéricos resultan más atractivos que los complejos modelos detallados de fabricantes para determinados tipos de simulaciones, UskiJoutsenvuo y Niskanen (2012). Sin embargo, hay que señalar que este tipo de modelos simplificados no deben ser utilizados en estudios de variaciones de frecuencia, así como en análisis de la respuesta de sistemas aislados o redes débiles, Mata Dumenjó et al. (2011).

\subsubsection{Antecedentes de la IEC 61400-27}

Modelos genéricos, con una estructura disponible de manera pública, han sido requeridos por bastantes compañías eléctricas desde hace unos años en Estados Unidos, Fortmann (2010); Fortmann et al. (2010). De esta manera, se puede considerar que el reciente trabajo que ha comenzado a desarrollar el Comité Técnico de la IEC 61400-27 toma como fuente de partida los esfuerzos llevados a cabo por el "Western Electricity Coordinating Council (WECC)" "Renewable Energy Modeling Task Force (REMTF)" (anteriormente conocido como "Wind Generator Modeling Group (WGMG)"), WECC REMTF (2010); NERC (2010); Ellis et al. (2011a b). 
La primera reunión del Comité Técnico responsable de la IEC 61400-27 se celebró en octubre del año 2009 en Dinamarca. Actualmente, se compone de 43 miembros pertenecientes a 16 países (Austria, Alemania, China, Corea, Dinamarca, España, Estados Unidos, Francia, Finlandia, Gran Bretaña, Irlanda, Japón, Noruega, Países Bajos, Rusia y Suecia), Sørensen et al. (2012). Estos miembros representan a compañías y organizaciones interesadas en el desarrollo de la Norma, incluyendo operadores del sistema eléctrico, fabricantes de aerogeneradores, empresas de software de simulación de sistemas eléctricos de potencia, así como diversos grupos de investigación. Precisamente, el responsable del Comité Técnico español es uno de los directores de la presente Tesis Doctoral.

Un total de 13 reuniones han tenido lugar hasta la fecha desde el año 2009, Honrubia Escribano et al. (2012c). Durante el año 2010, las reuniones se centraron en el procedimiento de validación de los modelos. De esta manera, en 2011 la tarea principal del Comité Técnico fue la definición de los modelos de la Parte 1. A finales del año 2011 la versión CD de la Norma fue enviada a los comités nacionales para obtener los comentarios de éstos. Las reuniones celebradas en el transcurso de 2012 han servido para iniciar el desarrollo de la Parte 2 de la citada Norma y, al mismo tiempo, se están ultimando determinados aspectos de la Parte 1 en relación con la definición de determinados aspectos de los modelos de aerogeneradores. En base a este cronograma, representado en la figura 1.7, una versión impresa de la Norma no se espera que sea publicada antes de mediados del año 2013 para la Parte 1, y habrá que esperar hasta 2015 para la Parte 2, Sørensen et al. (2011).

Con respecto a la Parte 1, se han definido cuatro topologías de aerogeneradores, figura 4.1, de acuerdo a las configuraciones más empleadas en el sector eólico:

- Tipo 1: aerogenerador directamente conectado a la red eléctrica a través de un generador asíncrono de jaula de ardilla. Esta tipología representa la solución tecnológica más antigua que se puede encontrar en los parques eólicos, Keung et al. (2009). Dado que está compuesta por una máquina de inducción convencional, su rango de velocidades es muy reducido y posee un carácter inductivo, Ellis et al. (2011b). Por este motivo es usual encontrarse una batería de condensadores para compensar el factor de potencia, tal y como se muestra en la figura 4.1a; similar a la ensayada en el apartado 2.4.1.4, aunque de mayor capacidad. También, en la mayoría de las ocasiones esta configuración emplea un arrancador suave que se activa durante la conexión de la máquina para limitar la elevada intensidad transitoria que se produce durante la conexión del generador, Rodriguez Amenedo et al. (2003); González Rodríguez (2006). De esta tecnología se pueden encontrar tanto sistemas con ángulo de pitch fijo, como variable, con el objetivo de regular el ángulo de giro de las palas para evitar su aceleración durante 
un hueco de tensión, Lin et al. (2009); Thet y Saitoh (2009).

- Tipo 2: similar al tipo 1, aunque el rotor de la máquina está bobinado permitiendo, de esta manera, variar su resistencia, figura 4.1b. Con esta modificación se consigue variar el deslizamiento en un rango del $10 \%$, Burnham et al. (2009). Este tipo de aerogeneradores suelen emplear sistemas de regulación del ángulo de pala.

- Tipo 3: aerogenerador doblemente alimentado, coloquialmente conocido como "Doubly Fed Induction Generator (DFIG)", donde el estator se encuentra directamente conectado a la red de suministro y el rotor se conecta a través de un convertidor de potencia - el diagrama de construcción de este elemento es similar al mostrado en la figura 2.8 En relación con su respuesta ante huecos de tensión, existen soluciones que emplean un dispositivo conocido como "crowbar" para proteger al convertidor durante perturbaciones en la red de suministro, Fortmann et al. (2010); Jiménez Buendía y Barrasa Gordo (2012), aunque otros fabricantes emplean dispositivos conocidos como "braking chopper" conectados en la etapa de continua del convertidor, Timbus et al. (2011). Esta tecnología permite un rango de velocidades considerablemente superior que los tipos 1 y 2 . Es importante tener en cuenta que este tipo de generadores son empleados en la mayoría de los aerogeneradores que se encuentran actualmente instalados en Norte América, Zavadil et al. (2005); así como en el resto del mundo, Salles et al. (2010); Hiskens (2012). Aún con todo lo anterior, actualmente el Comité Técnico de la IEC no ha alcanzado un acuerdo sobre el modelo definitivo de generador tipo 3, Honrubia Escribano et al. (2012c). De hecho, existe una discusión abierta entre el grupo WECC REMTF y el de la IEC sobre la necesidad de incorporar el "crowbar" en las simulaciones, Pourbeik (2012a b); Jiménez Buendía y Barrasa Gordo (2012).

- Tipo 4: aerogenerador conectado a la red de suministro a través de un convertidor. Precisamente, esta solución se conoce como "full converter" debido a que toda la potencia generada atraviesa al convertidor, figura 4.1d. De esta manera la potencia generada por el rotor eólico a cualquier frecuencia es transformada en la frecuencia constante que posea la red de suministro a la que se conecta. Este sistema también permite un amplio rango de velocidades para la extracción de la máxima energía del viento. Con todo ello, esta tecnología, junto con el tipo 3 , son de las que se espera una mayor presencia en el sector eólico a nivel internacional en los próximos años, Langlois et al. (2009); Pourbeik (2012b).

Para finalizar el presente apartado, dedicado a la exposición de aspectos generales de los modelos simplificados, la tabla 4.1 nombra algunas de las 


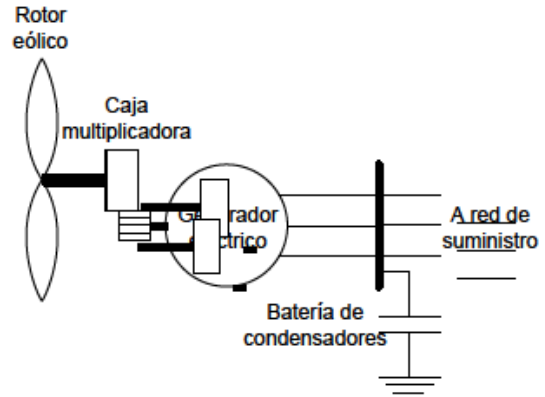

(a) Tipo 1.

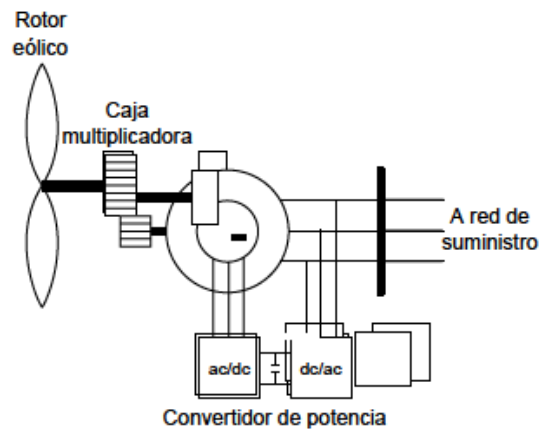

(c) Tipo 3 .

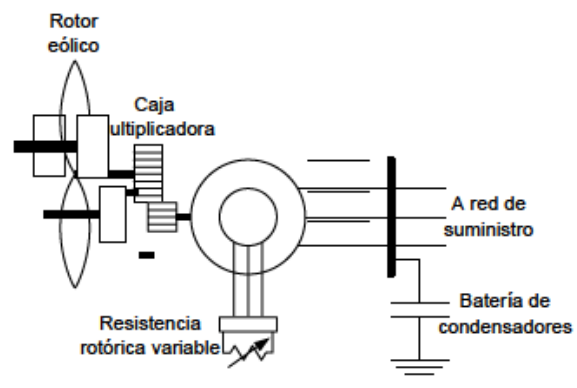

(b) Tipo 2 .

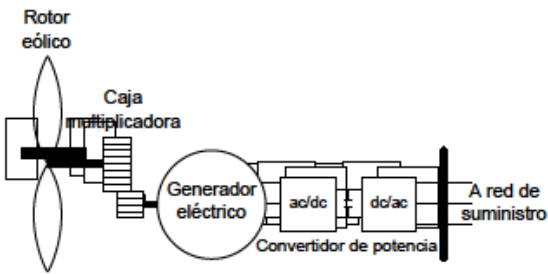

(d) Tipo 4 .

Figura 4.1: Tipologías de aerogeneradores más empleadas. 


\begin{tabular}{|c|c|c|c|c|}
\hline Tipo 1 & Tipo & & Tipo 3 & Tipo 4 \\
\hline $\begin{array}{l}\text { Vestas V82, } \\
1.65 \mathrm{MW}\end{array}$ & $\begin{array}{l}\text { Vestas } \\
1.8 \mathrm{MW}\end{array}$ & V80, & $\begin{array}{l}\text { General Electric, } \\
1.5 \mathrm{MW}\end{array}$ & $\begin{array}{l}\text { Enercon E70, } \\
2.3 \mathrm{MW}\end{array}$ \\
\hline Bonus 1.3 MW & $\begin{array}{l}\text { Vestas } \\
660 \mathrm{~kW}\end{array}$ & V47, & $\begin{array}{l}\text { General Electric, } \\
3.6 \mathrm{MW}\end{array}$ & $\begin{array}{l}\text { General Electric, } \\
2.5 \mathrm{MW}\end{array}$ \\
\hline Bonus 2.3 MW & $\begin{array}{l}\text { Vestas } \\
1.75 \mathrm{MW}\end{array}$ & V66, & $\begin{array}{l}\text { Gamesa G80, } \\
\text { G87, G90, G97, } \\
\text { G114, } 2 \text { MW }\end{array}$ & $\begin{array}{l}\text { Siemens SWT } \\
2.3-93,2.3 \mathrm{MW}\end{array}$ \\
\hline $\begin{array}{l}\text { Mitsubishi } \\
\text { MWT1000A, } \\
1 \mathrm{MW}\end{array}$ & $\begin{array}{l}\text { Gamesa } \\
1.8 \mathrm{MW}\end{array}$ & G80, & $\begin{array}{l}\text { Nordex } \quad \mathrm{N} 80, \\
2.5 \mathrm{MW}\end{array}$ & $\begin{array}{l}\text { Clipper Liberty, } \\
2.5 \mathrm{MW}\end{array}$ \\
\hline $\begin{array}{l}\text { Suzlon S66, } \\
1.25 \mathrm{MW}\end{array}$ & $\begin{array}{l}\text { Suzlon } \\
2.1 \mathrm{MW}\end{array}$ & S88, & $\begin{array}{l}\text { REpower } \\
\text { MM82, MM92, } \\
\text { MM100, } 2 \text { MW }\end{array}$ & $\begin{array}{l}\text { Samsung, } \\
2.5 \mathrm{MW}\end{array}$ \\
\hline
\end{tabular}

Tabla 4.1: Versiones comerciales de las topologías de aerogeneradores más comunes.

versiones comerciales de diversos fabricantes de aerogeneradores que poseen las diversas topologías que se han listado anteriormente. Aunque en dicha tabla se ha mostrado el mismo número de versiones comerciales de aerogeneradores por cada tipo de modelo, actualmente el número de modelos tipo 3 y 4 que se comercializa es considerablemente superior a los tipos 1 y 2 , tal y como se señaló previamente.

En base a lo comentado anteriormente, en el presente capítulo de la Tesis Doctoral se van a describir diversos componentes de modelos simplificados de aerogeneradores definidos por la IEC 61400-27, en línea con lo publicado en Honrubia Escribano et al. (2012c). Además, se ha realizado una campaña de medidas en un parque eólico con el interés de caracterizar ciertos parámetros de los modelos. 


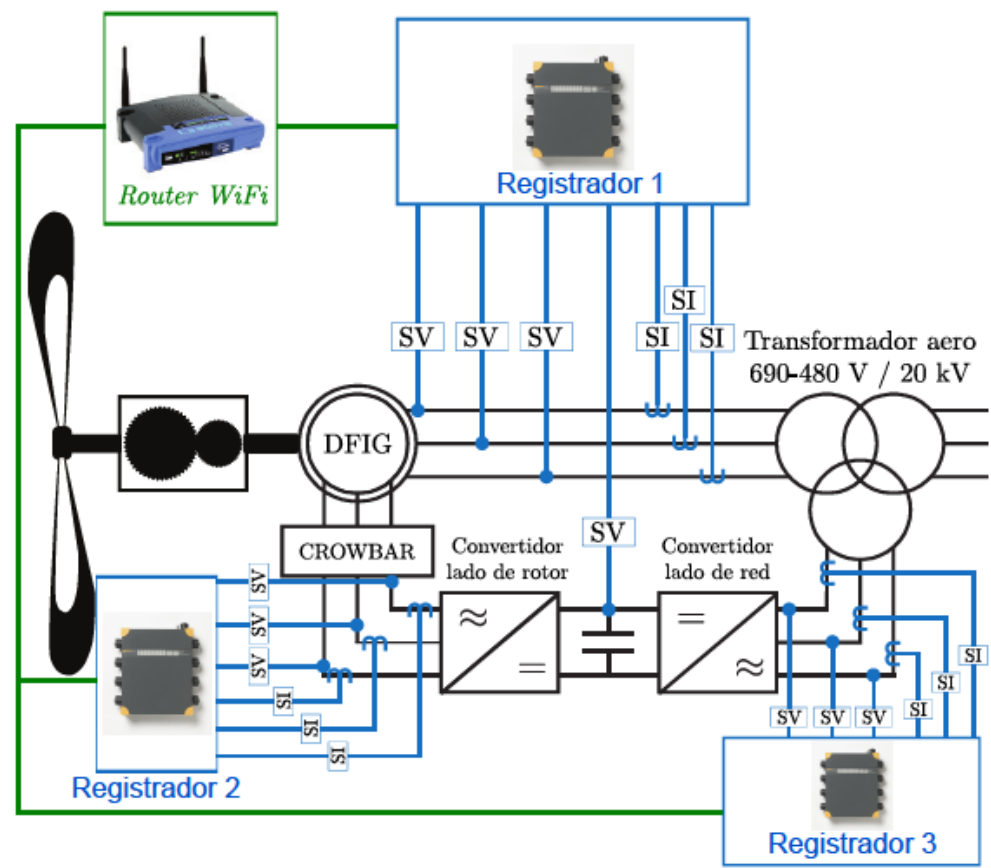

Figura 4.2: Instalación de los analizadores de calidad de energía eléctrica dentro de la góndola del aerogenerador 4 del parque eólico 3.

\subsection{Descripción de los ensayos y del material em- pleado}

Con el objetivo de conseguir parametrizar diversos componentes de los modelos simplificados desarrollados en el apartado 4.3 . se ha realizado una campaña de medidas en un parque eólico. Concretamente, se trata del parque eólico 3, descrito previamente en el apartado 3.2 .3 de la Tesis Doctoral.

En dicho parque se han instalado cuatro analizadores trifásicos de calidad de la energía eléctrica, específicamente el modelo 1760 de la marca Fluke. Tres de los analizadores se encuentran localizados en la góndola del aerogenerador número 4, dos de ellos conectados en el rotor y el restante en el estator del generador eléctrico. El cuarto registrador se encuentra conectado en la subestación a la que vierten energía los aerogeneradores del parque eólico.

En la figura 4.2 se muestra la disposición de los equipos de registro dentro de la góndola del aerogenerador. Sobre dicha figura se han señalado las sondas de tensión, SV, y de intensidad, SI, que emplean este tipo de registradores para monitorizar las tensiones e intensidades, respectivamente. También, puede verse que se ha instalado un router en la góndola del aerogenerador para permitir la descarga de datos desde la sala de control del parque eólico de manera inalámbrica. 
Además de la información registrada por los analizadores, tal y como se señaló en el apartado 3.2.3, se tiene acceso al sistema SCADA del parque eólico. De esta manera, las siguientes variables son registradas cada dos segundos: ángulo de pitch, intensidades de rotor y estator, frecuencia de red, tensión de red, temperatura del generador, dirección del viento, velocidad del viento, velocidad de giro del rotor eólico, potencia (activa y reactiva) generada, entre otras.

Los datos monitorizados por el SCADA comenzaron a registrarse desde el día 2 de noviembre de 2011. Además, teniendo en cuenta que han surgido diversos problemas con los analizadores Fluke, la disponibilidad de ambas fuentes de información se encuentra considerablemente limitada.

Con lo anterior, la figura 4.3 muestra dos gráficos con la información registrada por ambos elementos. Por un lado, la figura 4.3a representa cinco variables registradas por el sistema SCADA del aerogenerador número 4, el 20 de noviembre de 2011, desde las $13 \mathrm{~h}$ hasta las $14 \mathrm{~h}$ :

- Potencia reactiva: medida en kVAr, con un factor de escala igual a 20.

- Potencia activa: medida en kW.

- Tensión: medida en la parte de baja tensión del transformador del aerogenerador.

- Velocidad del rotor eólico: medida en revoluciones por minuto, rpm, con un factor de escala igual a 50.

- Velocidad del viento: medida en $\mathrm{m} / \mathrm{s}$, con un factor de escala de 50.

Por otro lado, la figura 4.3b representa el valor eficaz de las tres tensiones registradas en el estator del generador eléctrico del aerogenerador, durante un hueco de tensión trifásico ocurrido a las 13:18:42 horas.

Sobre la figura 4.3 se observa que, debido al hueco de tensión, el aerogenerador pasa de generar energía reactiva a consumirla; lo que se encuentra directamente relacionado con la actuación del crowbar en este tipo de aerogeneradores doblemente alimentados. 


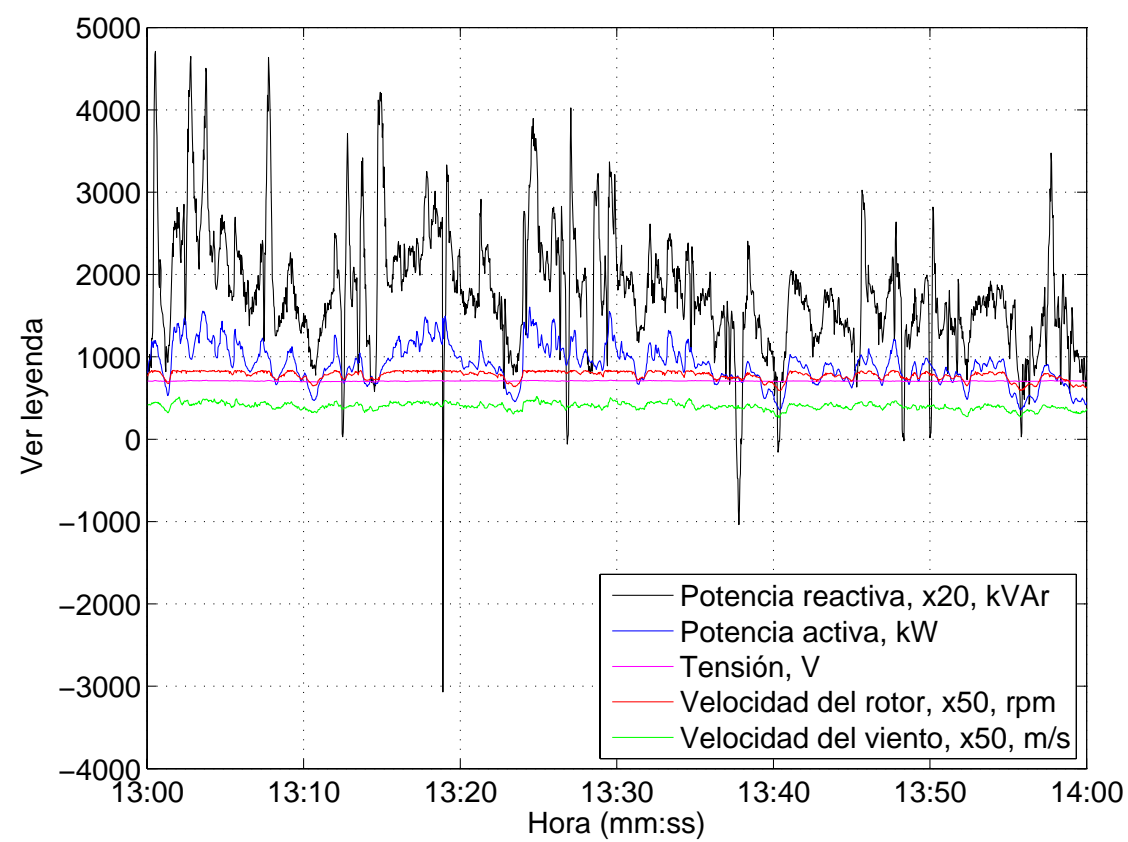

(a) Variables registradas por el SCADA.

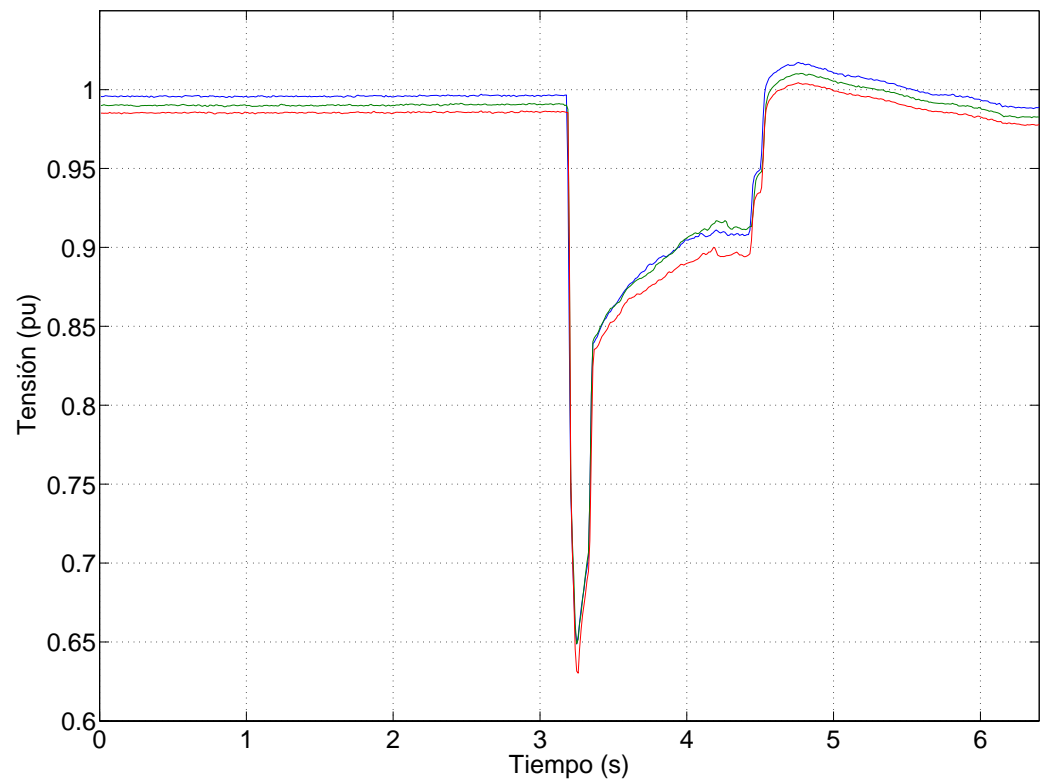

(b) Tensiones registradas por el analizador Fluke.

Figura 4.3: Ejemplo de magnitudes registradas por los equipos instalados en el parque eólico 3 durante un hueco de tensión. 


\subsection{Desarrollo de modelos simplificados de aeroge- neradores}

Una vez introducido el estado del arte en relación con los modelos simplificados de aerogeneradores en el apartado 4.1. y comentada la campaña de medidas realizada, apartado 4.2 en el presente apartado se van a desarrollar determinados modelos genéricos de diferentes subsistemas del aerogenerador, Honrubia Escribano et al. (2012c). Concretamente, se van a estudiar aquellos modelos que han conseguido un elevado grado de aceptación dontro del Comité Técnico de la IEC 61400-27, como es el caso del moc námico, el mecánico, y el de control de pitch. Con los datos recogidos en parque eólico 3 se van a obtener algunos de los parámetros requeridos por los modelos;

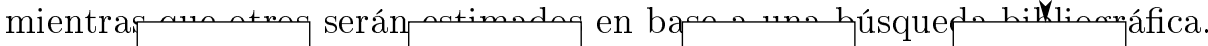

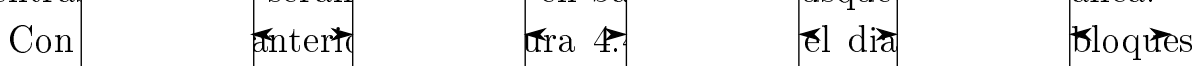
general que emplea cualquier modelo simplinc do do que sementaron en el apartado 4.1.1 y se mostraron en la figura 4.1. En la zona media de la

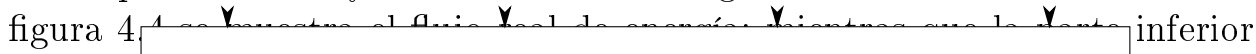
contiene aeroge-

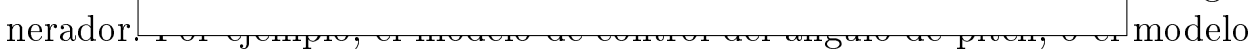
de control de la resistencia del rotor, son algunos de los controles que deben ser modelados.

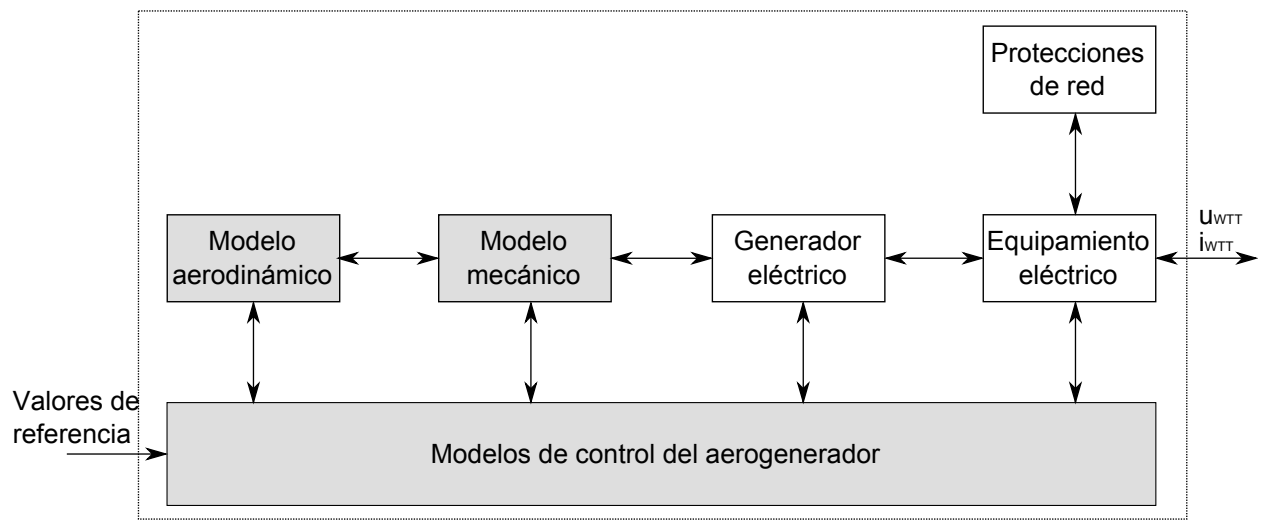

Figura 4.4: Diagrama de bloques general de los modelos simplificados.

Sobre la figura 4.4 se observa que los modelos de aerogeneradores toman la tensión de red, $u_{W T T}$, como variable de entrada y generan las intensidades, $i_{W T T}$, como variables de salida. Debido a que los aerogeneradores pueden recibir valores de referencia durante su operación normal dentro de un parque eólico, esta información también es recogida por los modelos. Concretamente, se consideran valores de referencia de potencia activa, reactiva, y de tensión.

Sin embargo, hay que tener en cuenta que, en función del tipo de modelo 
de aerogenerador de que se trate, es posible que no contenga algunos de los bloques de la figura 4.4. Por ejemplo, para aerogeneradores tipo 1 con ángulo de paso de pala fijo no se incluye ningún modelo de control del aerogenerador. También, de manera similar, el modelo tipo 2 no incluye un modelo aerodinámico; por lo que este modelo implementa un control adicional para calcular la potencia aerodinámica, en línea con lo publicado previamente por el grupo WECC|REMTE, WECC REMTF (2010); Ellis et al. (2011a).

De esta manera, a continuación se desarrollan cada uno de los subsistemas representados en la figura 4.4 en los que el Comité Técnico de la IEC 6140027 ha alcanzado un acuerdo sobre su utilización en estudios de estabilidad de los sistemas eléctricos de potencia. En concreto, los modelos analizados se han señalado con un fondo gris en la figura 4.4 .

\subsubsection{Modelo aerodinámico}

La principal función del modelo aerodinámico es obtener la potencia mecánica — también conocida como potencia aerodinámica- que se transmite al eje del rotor eólico a partir de la energía que lleva el viento, de acuerdo con la ecuación de conversión de la energía eólica (3.1). Tal y como se señaló en el apartado 3.1.1 de la Tesis Doctoral, $C_{p}$ es el coeficiente de potencia, que depende del parámetro adimensional "velocidad específica", $\lambda$, y del ángulo de paso de las palas, $\theta$. A su vez, $\lambda$ representa la relación entre la velocidad de punta de pala y la velocidad del viento incidente.

De esta manera, se puede señalar que la complejidad del modelo aerodinámico se encuentra determinada por el grado de detalle empleado para representar $C_{p}$. Uno de los métodos más precisos para obtener $C_{p}$ consiste en representarlo a través de un gráfico tridimensional, figura 4.5 . Price y Sanchez-Gasca (2006). Como puede verse sobre dicha figura, conforme aumenta el ángulo de paso de las palas se reduce $C_{p}$, es decir, se reduce la potencia extraída del viento. Para observar con mayor claridad la variación de $C_{p}$ dentro del rango de operación de un aerogenerador, la figura 4.6 muestra con una línea verde el valor de $C_{p}$ en función de $\lambda$ y $\theta$, Fortmann (2010).

Sin embargo, tal nivel de precisión penaliza el tiempo de simulación del modelo y, además, este grado de detalle resulta muy elevado para estudios de estabilidad del sistema eléctrico. En resumen, existen varias razones para emplear modelos aerodinámicos simplificados, Behnke et al. (2007):

- Tal y como se ha visto en la figura 4.5 el coeficiente $C_{p}$ es una función altamente no lineal de $\lambda$ y $\theta$. Por lo tanto, la resolución detallada de estas ecuaciones conlleva un proceso iterativo que implica una considerable cantidad de tiempo.

- Las características del perfil de pala son específicas de cada fabricante y, además, suelen ser confidenciales. 


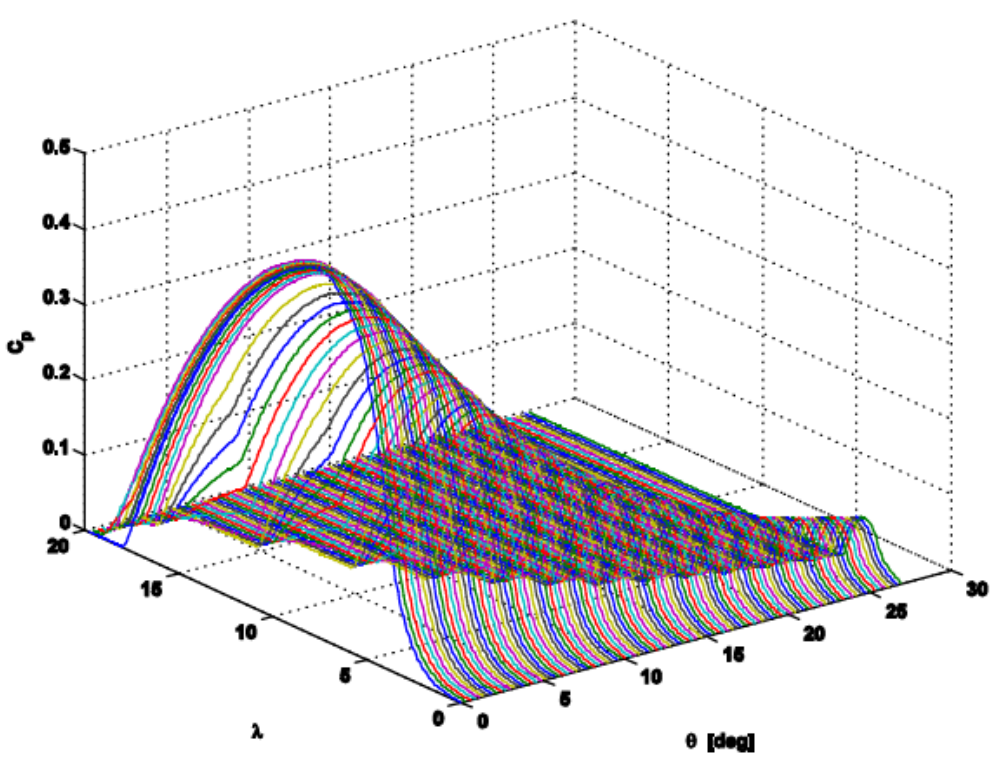

Figura 4.5: Representación tridimensional de $C_{p}$, Price y Sanchez-Gasca (2006).

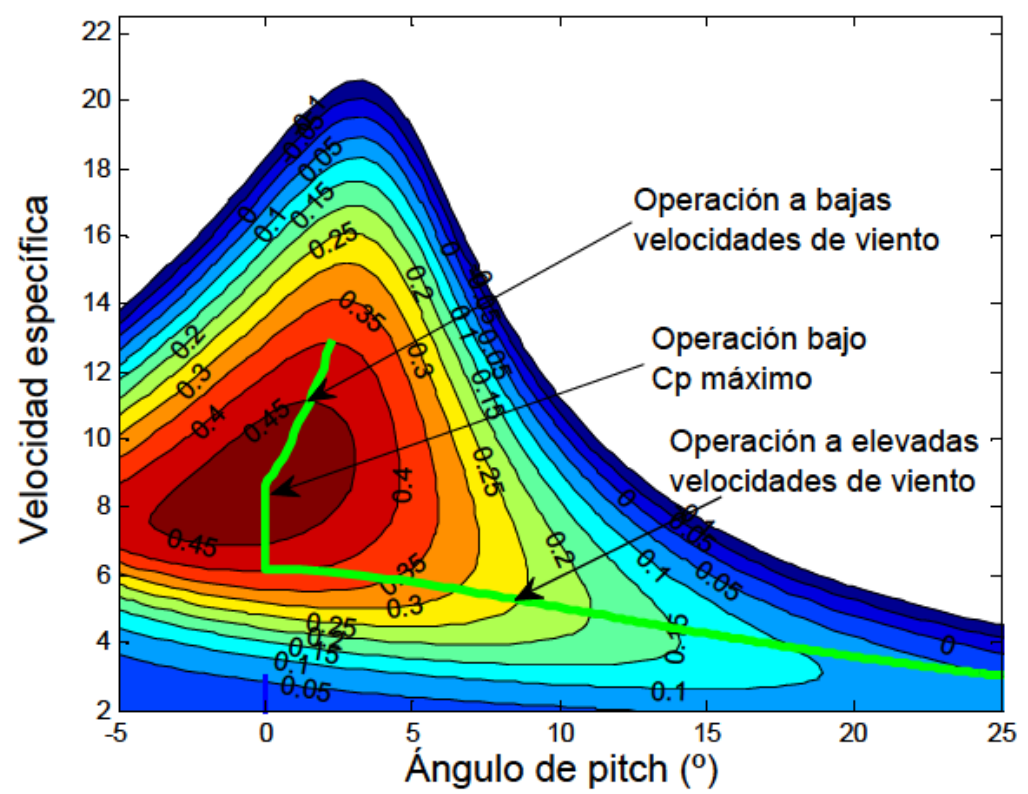

Figura 4.6: $C_{p}$ en función de $\lambda_{\mathrm{y}} \theta$, Fortmann (2010). 
Por lo tanto, si la velocidad del viento se considera constante, algunos autores han indicado que la relación entre $C_{p}, \lambda$ y $\theta$ se aproxima a una relación lineal en función de la variación de potencia con respecto al ángulo girado por las palas, $d p / d \theta$, Price y Sanchez-Gasca (2006). Según esta hipótesis, la potencia aerodinámica, $p_{\text {aero }}$, puede ser obtenida mediante la ecuación 4.1).

$$
p_{\text {aero }}=p_{\text {aero } 0}-\frac{d p}{d \theta} \cdot\left(\theta-\theta_{0}\right)
$$

Concretamente, en Price y Sanchez-Gasca (2006) se evalúa la respuesta de un modelo aerodinámico simplificado obtenido mediante (4.1), sobre dos aerogeneradores comerciales de diferentes potencias nominales del fabricante General Electric — señalados en la tabla 4.1-, utilizado para estudios de estabilidad transitoria. Con un tiempo de simulación entre 10 s y 20 s y, asumiendo que la velocidad del viento es constante en dicho intervalo, obtienen que el modelo aerodinámico simplificado se comporta de manera correcta en comparación con un modelo detallado del fabricante.

De manera similar, en Behnke et al. (2007) se emplea un modelo aerodinámico linealizado idéntico al de la ecuación (4.1), y lo compara añadiendo a la ecuación el término de variación de potencia con respecto a la velocidad de giro del rotor eólico, $d p / d \omega_{\text {rotor }}$. Concluye que este segundo término de la ecuación no tiene influencia apreciable sobre la potencia aerodinámica; lo que puede estar relacionado con que la elevada constante de inercia que posee el rotor eólico causa que una perturbación no modifique de manera considerable la velocidad de giro del mismo y, por lo tanto, el término $d p / d \omega_{\text {rotor }}$ pueda ser despreciable.

Sin embargo, en Fortmann (2010) se demuestra que el término $d p / d \omega_{\text {rotor }}$ tiene una influencia sobre la potencia aerodinámica que no puede ser despreciada. Mediante medidas en campo con aerogeneradores de $2 \mathrm{MW}$ y $6 \mathrm{MW}$ de potencia nominal, afirma que la contribución del término $d p / d \omega_{\text {rotor }}$ al cambio en la potencia aerodinámica ante un hueco de tensión se sitúa entre el $10 \%$ y el $15 \%$.

Por lo tanto, teniendo en cuenta las ideas anteriores, la versión CD de la Norma IEC 61400-27 contempla la implementación de un modelo aerodinámico linealizado, que calcula la potencia aerodinámica en función de los dos términos señalados en los párrafos anteriores, Honrubia Escribano et al. (2012c), ecuación (4.2). Los términos de dicha ecuación se han definido anteriormente y los subíndices " 0 " indican valores iniciales.

$$
p_{\text {aero }}=p_{0}-\frac{\partial p}{\partial \theta} \cdot\left(\theta-\theta_{0}\right)+\frac{\partial p}{\partial \omega_{\text {rotor }}} \cdot\left(\omega_{\text {rotor }}-\omega_{\text {rotor } 0}\right)
$$

Tal y como se observa en 4.2, para obtener la potencia aerodinámica, es necesario conocer los términos incluidos en las derivadas parciales; por lo que a continuación se muestra su desarrollo en línea con lo publicado en Honrubia Escribano et al. (2012c). 


\begin{tabular}{l|c|c}
\hline Referencia & $k_{\text {aero }}$ & $C_{\text {aero }}$ \\
\hline Fortmann $(2010)$ & -0.008 & $-0.06:-0.07$ \\
\hline WECC REMTF $(2010)$ & -0.007 & 0 \\
\hline Price y Sanchez-Gasca $(2006) ;$ Behnke et al. (2007) & -0.01 & 0 \\
\hline
\end{tabular}

Tabla 4.2: Valores de parámetros necesarios para calcular el término $\frac{\partial p}{\partial \theta}$.

\subsubsection{Obtención del término $\frac{\partial p}{\partial \theta}$.}

En el caso del término que muestra la variación de la potencia aerodinámica en función del ángulo de pitch, éste se puede representar a través de una función lineal. Por lo tanto, conociendo sólo dos parámetros, es decir, la pendiente, $k_{a e r o}$, y la ordenada en el origen, $C_{a e r o}$, de dicha función lineal, se está en condiciones de calcular el término $\frac{\partial p}{\partial \theta}$, dado que: $\frac{\partial p}{\partial \theta}=k_{\text {aero }} \cdot \theta_{0}+C_{\text {aero }}$. Por este motivo, se requiere a los fabricantes de aerogeneradores suministrar los valores de estos parámetros. Debido a que, en el momento de realizar la presente Tesis Doctoral no se conocen datos de fabricantes, se ha buscado información en la bibliografía científica. Con ello, en la tabla 4.2 se resumen los valores encontrados. Los señalados por Fortmann (2010) se refieren a valores típicos para aerogeneradores de potencia nominal comprendida entre 2 MW y 6 MW; mientras que los valores aportados por Price y SanchezGasca (2006); Behnke et al. (2007) se corresponden con valores aproximados de un aerogenerador del fabricante General Electric de 1.5 MW. También, los valores ofrecidos por WECC REMTF (2010), idénticos a Pourbeik (2012b), se corresponden con valores típicos de aerogeneradores de General Electric.

\subsubsection{Obtención del término $\frac{\partial p}{\partial \omega}$.}

En el caso del término que representa la variación de la potencia aerodinámica en función de la velocidad de giro del rotor eólico, $\frac{\partial p}{\partial \omega_{\text {rotor }}}$, éste no puede ser representado a través de una única ecuación lineal. Son necesarias varias funciones lineales, todas ellas dependientes de la velocidad del viento, $v_{w}$, tal y como muestra la figura 4.7. En línea con dicha figura, resulta necesario conocer cuál es el valor de la velocidad del viento antes de iniciar la simulación. Para obtener este valor, a raíz del flujo de cargas previo a la simulación, se parte del valor de la potencia eléctrica generada por el aerogenerador. De esta manera, si el parámetro "velocidad del viento para una potencia generada de $0.3 \mathrm{pu}$ ", $v_{W P 03}$, es definido por el fabricante, la velocidad del viento puede ser calculada en base a la curva de potencia del aerogenerador, concepto comentado en el apartado 3.1.3 de la Tesis Doctoral. Bajo esta hipótesis, en la región donde la potencia generada es inferior a $0.3 \mathrm{pu}$, la velocidad del viento se puede estimar de acuerdo a una relación 


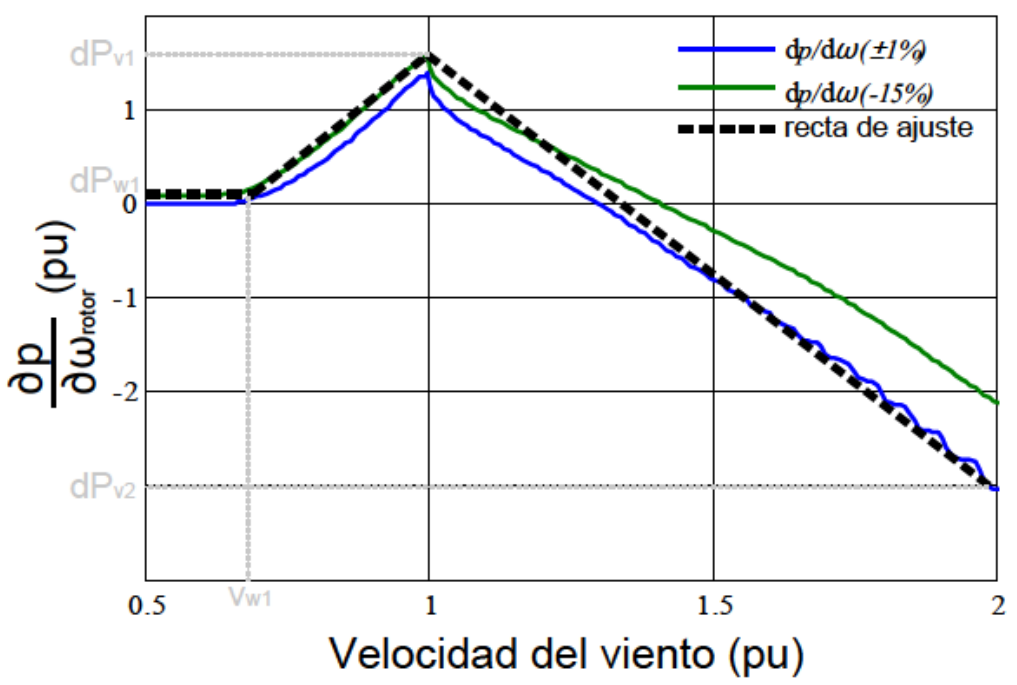

Figura 4.7: Representación del término $\frac{\partial p}{\partial \omega_{\text {rotor }}}$, Fortmann 2010).

cúbica definida por 4.3 ; mientras que si la potencia es superior a $0.3 \mathrm{pu} \mathrm{e}$ inferior a $1 \mathrm{pu}$, se puede emplear una aproximación lineal, descrita también en (4.3). Finalmente, para situaciones de altas velocidades de viento, dado que $p_{0} \geq 1$, la velocidad del viento se puede deducir si el parámetro "ángulo de pitch para dos veces la velocidad nominal del viento", $\theta_{V W 2}$, es dado por el fabricante, de acuerdo con 4.4.

$$
\begin{gathered}
v_{w}= \begin{cases}\sqrt[3]{\frac{P_{0} \cdot v_{W P 03}^{3}}{0,3}} & \text { si } p_{0}<0,3 \\
1+\frac{\left(p_{0}-1\right) \cdot\left(1-v_{W P 03}\right)}{0,7} & \text { si } 0,3 \leq p_{0}<1\end{cases} \\
v_{w}=\sqrt{\frac{4 \cdot \theta_{V W 2}}{4 \cdot \theta_{V W 2}-3 \cdot \theta_{0}}} \text { si } p_{0} \geq 1
\end{gathered}
$$

Una vez que la velocidad del viento es conocida mediante el empleo de las ecuaciones 4.3 ó 4.4, el término $\frac{\partial p}{\partial \omega_{\text {rotor }}}$ se puede deducir teniendo en cuenta las tres regiones que lo definen, figura 4.7 .

- Desde la velocidad de arranque del aerogenerador hasta la velocidad nominal de rotor, $v_{\omega 1}: \frac{\partial p}{\partial \omega_{\text {rotor }}}$ es constante y de valor reducido, $d P_{\omega 1}$. Esto es debido a que, en esta región, los cambios en la velocidad de giro del rotor no implican cambios sobre el ángulo de pitch.

- Desde la velocidad nominal de rotor hasta la velocidad nominal del aerogenerador: $\frac{\partial p}{\partial \omega_{\text {rotor }}}$ aumenta de forma lineal, hasta alcanzar el máximo, en $d P_{v 1}$. En esta zona tampoco se esperan cambios en el ángulo de pitch. 


\begin{tabular}{l|c|c|c|c}
\hline Entrada, $v_{w}(\mathbf{p u})$ & 0 & $v_{\omega 1}$ & 1 & 2 \\
\hline Salida, $\frac{\partial p}{\partial \omega_{\text {rotor }}}(\mathbf{p u})$ & $d P_{\omega 1}$ & $d P_{\omega 1}$ & $d P_{v 1}$ & $d P_{v 2}$ \\
\hline
\end{tabular}

Tabla 4.3: Parámetros necesarios para calcular el término $\frac{\partial p}{\partial \omega_{\text {rotor }}}$.

\begin{tabular}{l|c|c|c|c}
\hline Referencia & $d P_{\omega 1}$ & $v_{\omega 1}$ & $d P_{v 1}$ & $d P_{v 2}$ \\
\hline Fortmann $(2010)$ & $0.1: 0.2$ & $0.5: 0.7$ & $1.3: 1.6$ & $-2.5:-3.5$ \\
\hline
\end{tabular}

Tabla 4.4: Valores de parámetros necesarios para calcular el término $\frac{\partial p}{\partial \omega_{\text {rotor }}}$.

- Para velocidades de viento superiores a la nominal: $\frac{\partial p}{\partial \omega_{\text {rotor }}}$ disminuye de forma lineal, cuyo mínimo se obtiene en $d P_{v 2}$. Concretamente, la forma de la gráfica en esta región se encuentra directamente relacionada con la actuación del servomotor del pitch.

Por lo tanto, el impacto de la velocidad de giro del rotor sobre la potencia aerodinámica se puede resumir empleando una tabla que contenga los cuatro parámetros definidos anteriormente — señalados en color gris sobre la figura 4.7 - , tabla 4.3 . Debido a que no se conocen datos directamente revelados por fabricantes de los valores de los parámetros descritos en la tabla 4.3 es necesario buscar sus valores en la bibliografía, tal y como se muestra en la tabla 4.4. Teniendo en cuenta la disponibilidad de los datos registrados en el parque eólico descrito en los apartados 3.2 .3 y 4.2 de la Tesis Doctoral, el valor del parámetro $v_{\omega 1}$ se puede considerar igual a $0.67 \mathrm{pu}$.

Por último, para conseguir la solución completa del término $\frac{\partial p}{\partial \omega_{\text {rotor }}}$, se ha visto que es necesario obtener dos parámetros adicionales para obtener la velocidad del viento, $v_{W P 03}$ y $\theta_{V W 2}$. En función de los datos registrados en el parque eólico, se pueden estimar los siguientes valores:

- $v_{W P 03} 0.5756 \mathrm{pu}$. Este parámetro puede ser deducido a raíz de la curva de potencia que muestran públicamente los fabricantes de aerogeneradores.

- $\theta_{V W 2} 24.3328^{\circ}$. En relación con este parámetro, la figura 4.8 muestra la variación del ángulo de pitch en función de la velocidad del viento registrada en el aerogenerador 1 del parque eólico 3. En WECC REMTF (2010) se señala que el valor de $\theta_{V W 2}$ puede aproximarse a $21.98^{\circ}$.

Es importante señalar que el modelo aerodinámico simplificado que se ha desarrollado en el presente apartado es implementado por los modelos de aerogeneradores tipo 3 y 4, mencionados en el apartado 4.1.1. En el caso 


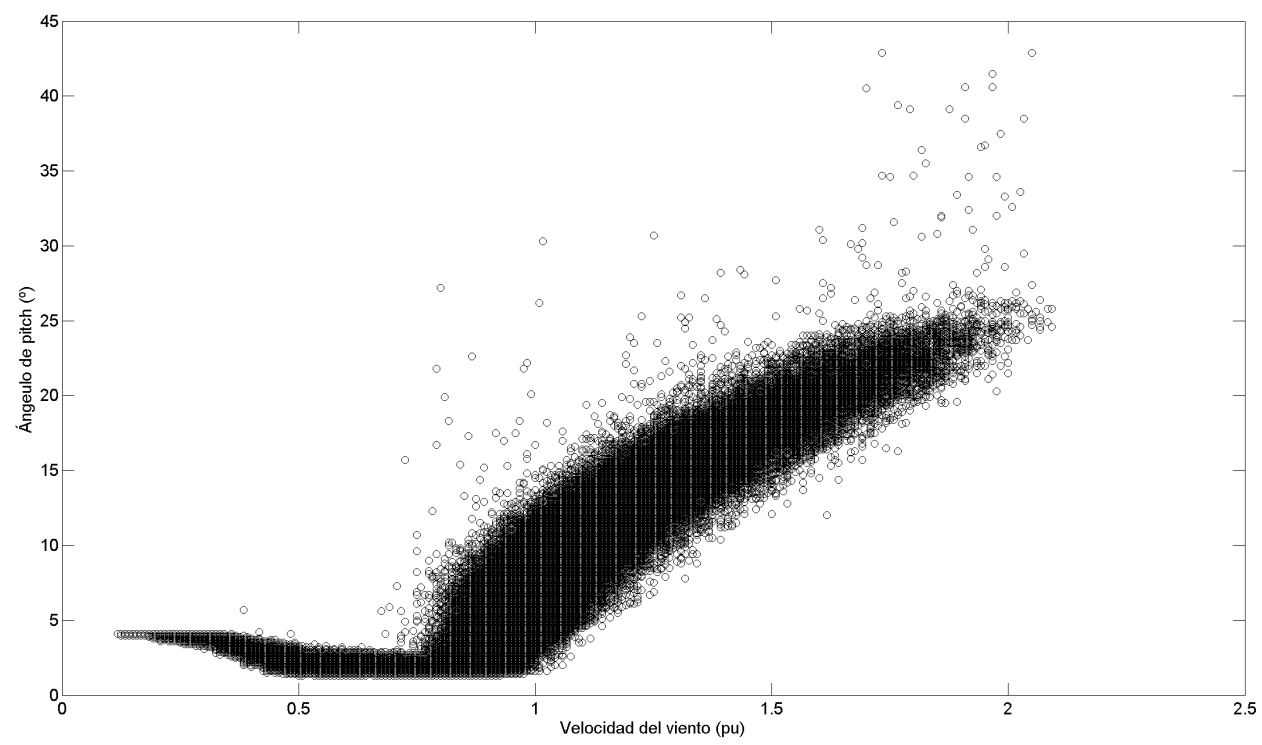

Figura 4.8: Variación del ángulo de pitch de un aerogenerador en función de la velocidad del viento registrada en el parque eólico 3 .

del modelo tipo 1 con ángulo de paso de pala fijo, el Comité Técnico de la IEC 61400-27 ha considerado que el par aerodinámico es constante durante la simulación, Sørensen et al. (2011).

Finalmente, la figura 4.9 muestra la implementación de todo el modelo aerodinámico que se ha desarrollado en el presente apartado. Tal y como se observa en la figura 4.9, la parte superior del modelo aerodinámico incluye la contribución de la variación del ángulo de pitch sobre la potencia aerodinámica; mientras que la parte inferior incluye la aportación de la variación de la velocidad de giro del rotor eólico. De manera similar, en la zona media del diagrama de bloques se observa el procedimiento de cálculo de la velocidad del viento a raíz de la potencia eléctrica inicial que se está generando, $p_{0}$ — dado que este dato es información de partida para el modelo-.

Una vez que se ha presentado el modelo aerodinámico simplificado que emplea actualmente la versión CD de la IEC 61400-27, figura 4.9, se puede comparar con el desarrollado previamente por el grupo WECC REMTE La diferencia más relevante consiste en que el modelo aerodinámico simplificado del WECC REMTF no incluye el término de variación de la potencia aerodinámica con respecto a la velocidad de giro del rotor, $\frac{\partial p}{\partial \omega_{\text {rotor }}}$; por lo que el modelo es considerablemente más sencillo al no necesitar conocer la velocidad del viento. También, otra diferencia es que los valores que el grupo WECC REMTF ha aportado del parámetro $C_{\text {aero }}$ son nulos, es decir, este grupo no tiene en cuenta el offset en el cálculo del parámetro $\frac{\partial p}{\partial \theta}$. 


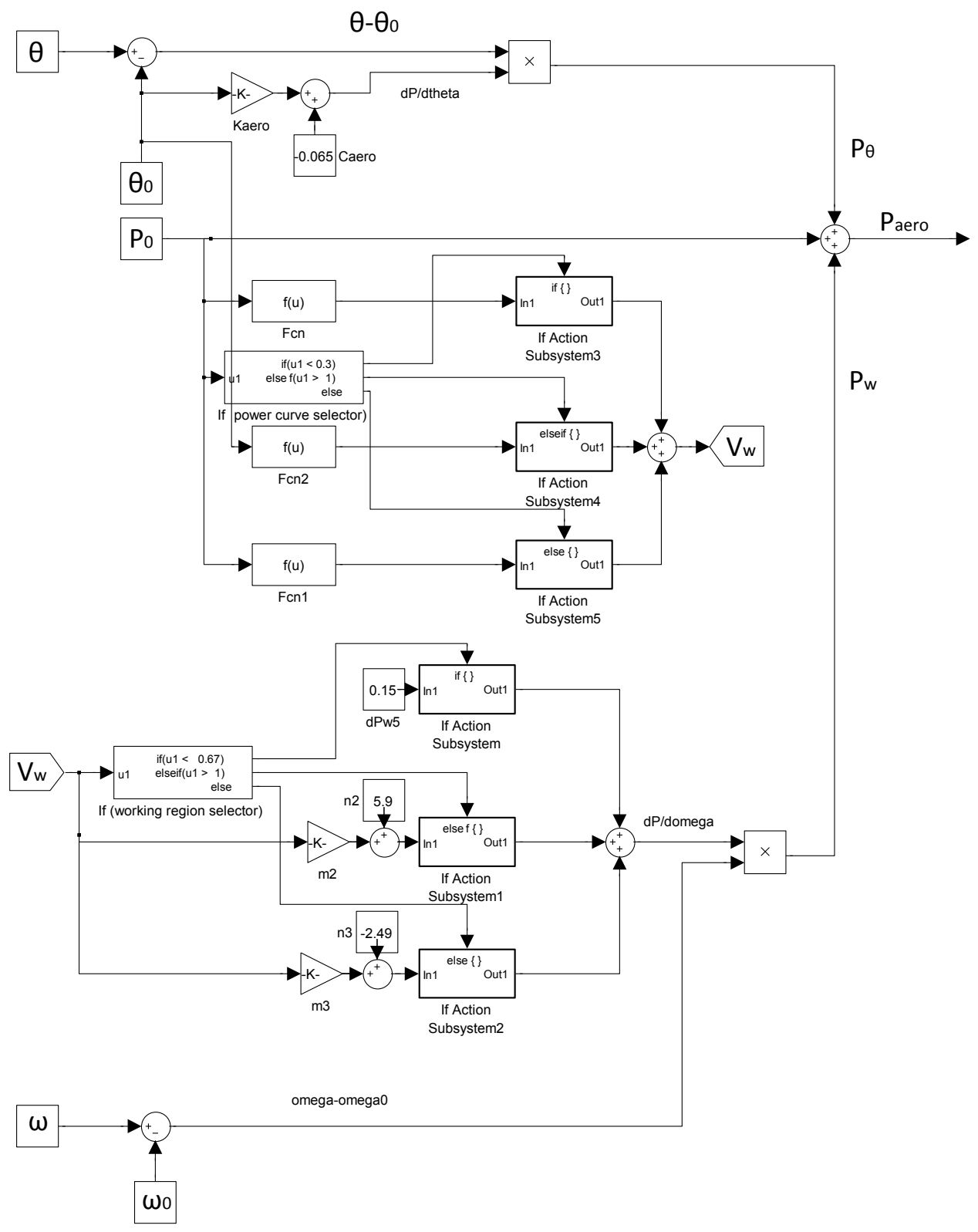

Figura 4.9: Implementación del modelo aerodinámico simplificado. 


\subsubsection{Modelo mecánico}

El sistema mecánico de un aerogenerador, también conocido como sistema de transmisión o tren de potencia o "drivetrain system", está compuesto por el conjunto de masas rotativas y los ejes que conectan dichas masas. Adicionalmente se suelen encontrar cajas multiplicadoras, conocidas como "gear box", debido a la diferencia existente entre las velocidades del eje de baja velocidad unido al rotor eólico y el eje de alta velocidad unido al generador eléctrico.

Para modelar este sistema, en la bibliografía se puede encontrar una diversidad de modelos mecánicos, desde aquéllos que simplifican todo el sistema en una sola masa a los más complejos que emplean hasta seis masas, Muyeen et al. (2007). Las motivaciones para desarrollar modelos mecánicos simplificados son similares a las que se señalaron en el apartado 4.3.1 para el modelo aerodinámico simplificado. Por un lado, prácticamente cada fabricante emplea un tipo de modelo mecánico específico (una masa, dos masas, etc.) y, además, resulta muy complicado acceder a dicha información, Behnke et al. (2007). En Ellis et al. (2011a) se señala que los modelos mecánicos deben de poder ser representados mediante una única masa o dos masas. De hecho, esta hipótesis es la que maneja actualmente el grupo WECCIREMTF, Ellis et al. (2011b). En esta línea, en Langlois et al. (2009) se señala que, al menos, debe emplearse un modelo de dos masas en simulaciones relacionadas con la estabilidad de sistemas de potencia. Sin embargo, es importante tener en cuenta que la red eléctrica sobre la que se centra Langlois et al. (2009) es una red relativamente débil (es decir, su capacidad de transporte de energía eléctrica es baja) y, en estos casos, la precisión de los modelos debe ser más elevada, tal y como se comentó en el apartado 4.1 .

Con lo anterior, el Comité Técnico de la IEC 61400-27 ha aceptado un modelo de dos masas para definir el modelo mecánico de todos los tipos de aerogeneradores señalados en el apartado 4.1.1. Sørensen et al. (2011). El modelo de dos masas agrupa todo el sistema de transmisión en dos masas unidas por un eje, tal y como se ha representado en la figura 4.10. La masa de la izquierda representa las palas, el buje y el eje lento; mientras que la derecha simboliza el eje rápido, que está básicamente constituido por un acoplamiento elástico y la máquina eléctrica.

De esta manera, el modelo mecánico de dos masas responde a la ecuación (4.5), Honrubia Escribano et al. (2012c).

$$
\begin{aligned}
& 2 H_{\text {rotor }} \frac{d \omega_{\text {rotor }}}{d t}=T_{\text {aero }}-d_{\text {sh }}\left(\omega_{\text {rotor }}-\omega_{\text {gen }}\right)-k_{\text {sh }}\left(\theta_{\text {rotor }}-\theta_{\text {gen }}\right) \\
& 2 H_{\text {gen }} \frac{d \omega_{\text {gen }}}{d t}=d_{\text {sh }}\left(\omega_{\text {rotor }}-\omega_{\text {gen }}\right)+k_{\text {sh }}\left(\theta_{\text {rotor }}-\theta_{\text {gen }}\right)-T_{\text {gen }}
\end{aligned}
$$

Tal y como se observa en (4.5), al igual que en la figura 4.10, el par mecánico - éste se obtiene directamente del modelo aerodinámico descrito 


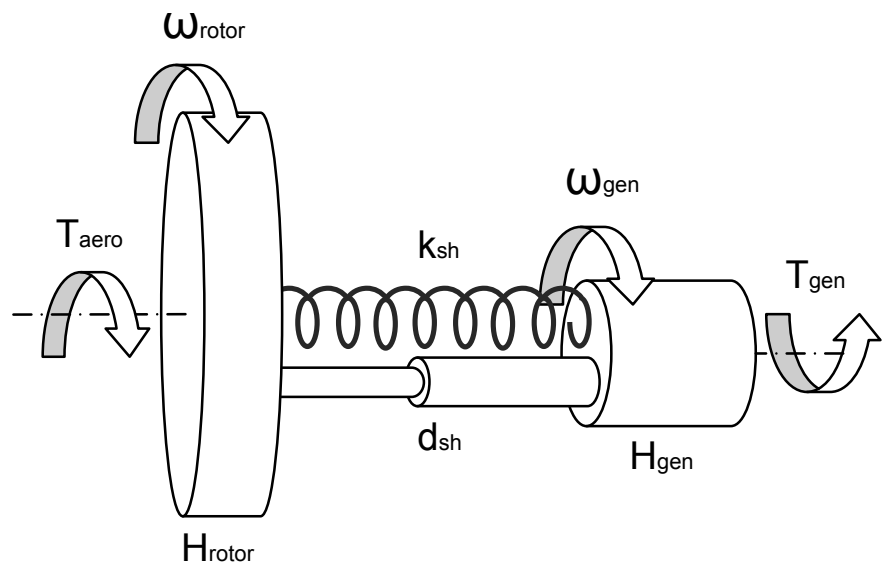

Figura 4.10: Esquema general de un modelo mecánico de dos masas.

en el apartado 4.3.1,$T_{\text {aero }}$, se aplica directamente sobre la inercia del rotor eólico para acelerarlo. De manera similar, el par del generador eléctrico también conocido como par electromagnético-,$T_{g e n}$, resiste la aceleración del generador. La diferencia entre las velocidades del rotor, $\omega_{\text {rotor }}$, y del generador, $\omega_{\text {gen }}$, causa, por un lado, la torsión del eje en una magnitud proporcional al coeficiente de torsión, $k_{s h}, \mathrm{y}$, al mismo tiempo, existe un coeficiente de amortiguamiento, $d_{s h}$, que también se resiste a la diferencia de velocidades. Por último, en 4.5 se observa que es necesario tener en cuenta las constantes de inercia del rotor eólico y del generador, $H_{\text {rotor }}$ y $H_{\text {gen }}$, respectivamente; así como los ángulos girados por cada eje, $\theta_{\text {rotor }}$ y $\theta_{g e n}$.

En resumen, para poder simular el modelo mecánico de dos masas tal y como indica la IEC 61400-27 es necesario conocer una serie de parámetros, definidos en la tabla 4.5 . Aunque estos parámetros son requeridos a los fabricantes para la correcta representación de la respuesta de sus aerogeneradores en estudios de estabilidad de los sistemas de energía eléctrica, en la última columna de la tabla 4.5 se muestran valores típicos para aerogeneradores con una potencia nominal comprendida entre $1 \mathrm{MW}$ y $5 \mathrm{MW}$, Ackerman et al. (2005). De todas maneras, existen bastantes referencias donde se indican valores específicos de dichos parámetros para aerogeneradores comerciales, Perdana (2008); WECC REMTF (2010); Clark et al. (2010); Pourbeik (2012b).

No obstante, el cálculo de determinados parámetros mostrados en la tabla 4.5 requieren el conocimiento de algunas nociones sencillas; por lo que a continuación se muestra cómo se pueden estimar, Honrubia Escribano et al. (2012c). Las constantes de inercia se obtienen a partir de los momentos de inercia — este último parámetro depende exclusivamente de la geometría y distribución de la masa del elemento-, designados habitualmente por la variable " $J$ ". Por ejemplo, según Morren et al. (2006), en el caso del rotor 


\begin{tabular}{l|c|l|c}
\hline Símbolo & Unidad & Descripción & Valores típicos \\
\hline$H_{\text {rotor }}$ & $\mathrm{s}$ & $\begin{array}{l}\text { Constante de inercia del rotor } \\
\text { eólico. }\end{array}$ & $2.0-6.0$ \\
\hline$H_{\text {gen }}$ & $\mathrm{s}$ & $\begin{array}{l}\text { Constante de inercia del gene- } \\
\text { rador eléctrico. }\end{array}$ & $0.4-0.8$ \\
\hline$k_{\text {sh }}$ & $\mathrm{pu}$ & Coeficiente de rigidez del eje. & $0.3-0.6$ \\
\hline$d_{s h}$ & $\mathrm{pu}$ & $\begin{array}{l}\text { Coeficiente de amortigua- } \\
\text { miento del eje. }\end{array}$ & - \\
\hline
\end{tabular}

Tabla 4.5: Parámetros necesarios para modelar el sistema mecánico.

eólico, el momento de inercia se puede deducir de acuerdo a (4.6), donde $m_{r}$ representa la masa del rotor (incluye las tres palas) y $r$ representa el radio del rotor.

$$
J_{\text {rotor }}=\frac{1}{9} \cdot m_{r} \cdot r^{2}
$$

Una vez que se conocen los momentos de inercia, las constantes de inercia se calculan de acuerdo a 4.7), Ackerman et al. (2005); Morren et al. (2006); Kennedy et al. (2011). Sobre la expresión (4.7) se observa que la constante de inercia expresa la relación entre la energía cinética almacenada por el elemento en rotación y la potencia de dicho elemento. En Behnke et al. (2007) se indica que los aerogeneradores con potencias nominales, $P_{n}$, en el rango de MW poseen constantes de inercia de varios segundos, en línea con los valores señalados en la tabla 4.5. Su valor describe el tiempo durante el cual el generador podría generar su potencia nominal teniendo como única fuente de energía disponible la cinética almacenada en sus masas en rotación, Aparicio Marín (2011).

$$
H_{\text {rotor }}=\frac{J_{\text {rotor }} \cdot \omega_{\text {rotor }}^{2}}{2 \cdot P_{n}} \quad \text { o } \quad H_{\text {gen }}=\frac{J_{\text {gen }} \cdot \omega_{\text {gen }}^{2}}{2 \cdot P_{n}}
$$

Una vez realizada la descripción de los parámetros necesarios para obtener el modelo mecánico que adopta la IEC 61400-27, la figura 4.11 muestra el diagrama de bloques de dicho modelo, Honrubia Escribano et al. (2012c). Tal y como se observa sobre dicha figura y en línea con lo que se ha comentado en el presente apartado, las entradas al modelo mecánico son las potencias (o pares), aerodinámica y eléctrica; mientras que las salidas son las velocidades de giro, del rotor eólico y del generador.

Por último, una vez que se ha definido el modelo mecánico que emplea actualmente la versión CD de la IEC 61400-27, se puede comparar con el 


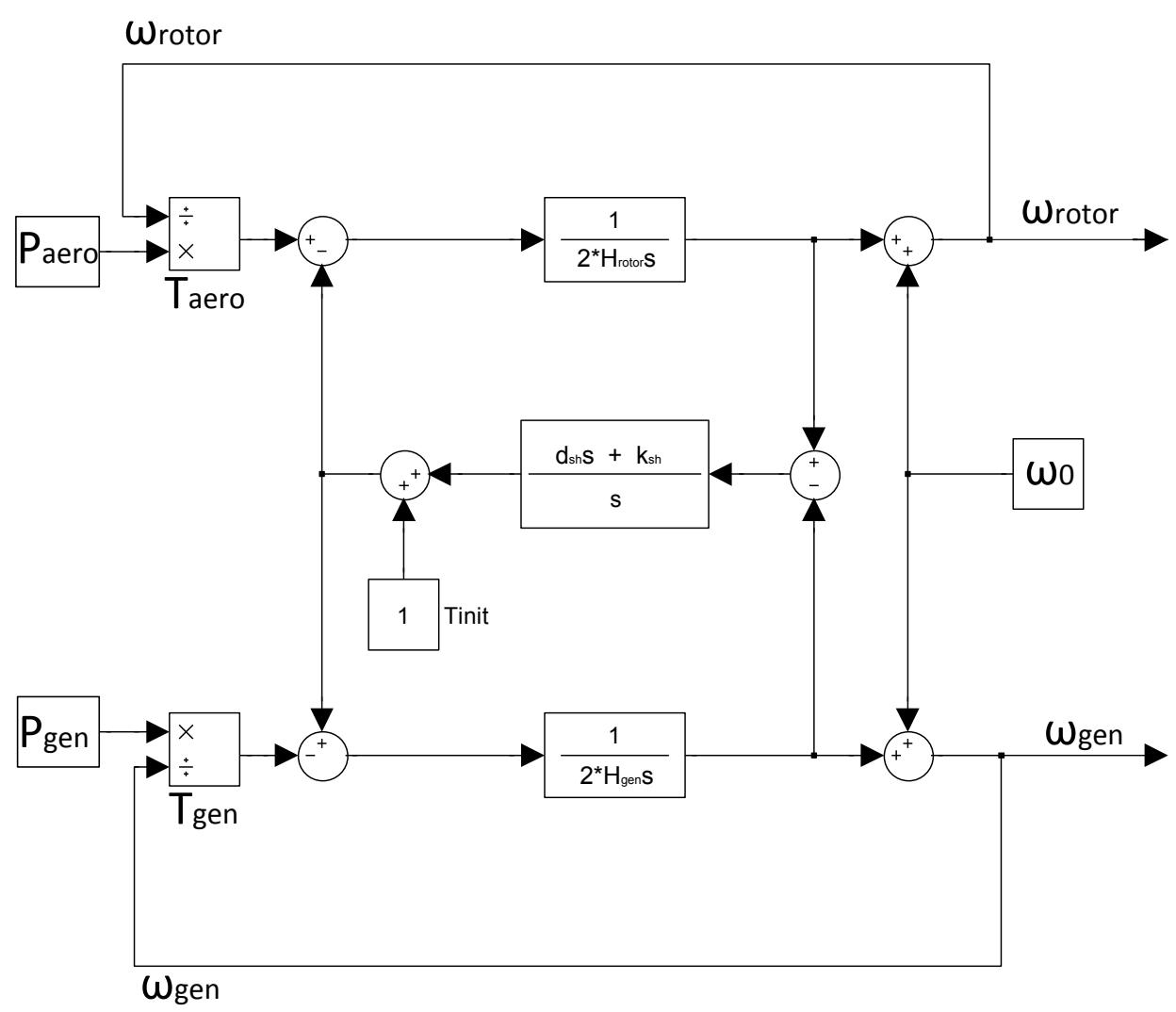

Figura 4.11: Esquema de bloques del modelo mecánico. 
desarrollado previamente por el grupo WECC REMTE. Se puede afirmar que ambos modelos son idénticos. El único aspecto a considerar es que el grupo WECCREMTF permite el empleo de un modelo de una masa en el modelo de aerogenerador tipo 1 definido en el apartado 4.1.1 mientras que el Comité Técnico de la IEC sólo incluye el modelo de dos masas.

\subsubsection{Modelo de control de pitch}

El ángulo de pitch de un aerogenerador - o ángulo de paso de las palaspermite controlar la potencia aerodinámica absorbida por el rotor eólico, en función de la velocidad del viento. Si ésta es superior al valor nominal, entonces se incrementa el ángulo girado por las palas para reducir la potencia extraída del viento, tal y como se mostró en las figuras 4.5 y 4.6. De manera similar, si la potencia eléctrica generada es inferior a la nominal, el controlador del ángulo de pitch debe situar el pitch en un valor, óptimo para la extracción de la máxima potencia aerodinámica, es decir, máximo $C_{p}$, Honrubia Escribano et al. (2012c), que se suele situar en los $0^{\circ}$, Lin et al. (2009).

Con las anteriores premisas, el grupo WECCIREMTF decidió implementar un modelo de control de pitch compuesto por dos controladores, uno que actúa sobre el error en la velocidad de giro y otro que actúa como compensador del error en la potencia eléctrica generada, WECC REMTF (2010); Ellis et al. (2011a); Hiskens (2012). Estos dos controladores implementados por el modelo han sido publicados previamente en Seyedi (2009); Lin et al. (2009); Langlois et al. (2009); Clark et al. (2010). De manera adicional, en el modelo de WECC se incluyen límites tanto en los valores máximo y mínimo que puede tomar el pitch, así como el límite de la velocidad de pitch máxima soportada por las palas del aerogenerador.

En línea con lo anterior, el modelo de control de pitch adoptado por el Comité Técnico de la IEC 61400-27 es idéntico al que ha desarrollado previamente el grupo WECC REMTF, con la salvedad de que en la versión de la IEC se ha incluido un parámetro adicional que relaciona los dos controladores definidos anteriormente, Honrubia Escribano et al. (2012c). Sin embargo, hay que señalar que la última modificación del modelo de control de pitch del grupo WECCIREMTF también incluye la ganancia cruzada, $K_{p x}$, debido precisamente al intercambio de información entre ambos grupos de trabajo, Pourbeik (2012b).

En resumen, la figura 4.12 muestra el modelo de control de pitch aceptado por la IEC 61400-27, Honrubia Escribano et al. (2012c). Tal y como se observa en la figura 4.12, el modelo de control de pitch se puede activar de dos formas diferentes:

- La manera habitual de activar el control es cuando la velocidad de giro del rotor alcanza su valor de referencia, $\omega_{\text {ref }}$, dado que una vez 


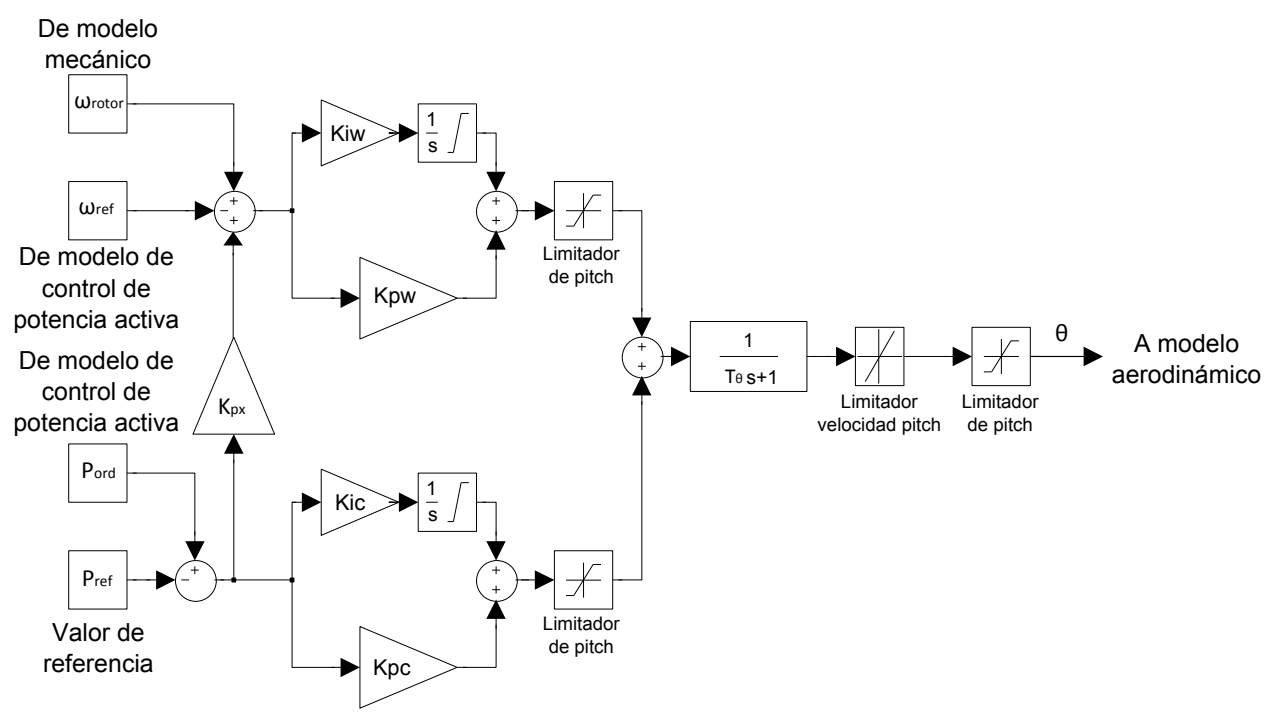

Figura 4.12: Esquema de bloques del modelo de control de pitch.

sobrepasado este valor las palas comienzan a modificar su pitch, en línea con lo comentado en el apartado 4.3.1.

- Dado que una perturbación en la red eléctrica, como un hueco de tensión, ocasiona que la potencia eléctrica disminuya y aumente la velocidad de giro del rotor eólico; también se produce la activación de dicho control.

Por lo tanto, para la implementación del modelo de control de pitch, es necesario conocer una serie de parámetros, que se han sintetizado en la tabla 4.6. En WECC REMTF (2010) se dan valores típicos para todas las variables mostradas en dicha tabla, excepto para $K_{p x}$.

El ángulo de pitch máximo se puede estimar igual a $27^{\circ}$, Clark et al. (2010); WECC REMTF (2010); Hiskens (2012). Gracias a la campaña de medidas realizada en la presente Tesis Doctoral el ángulo máximo de pitch que se ha registrado en el parque eólico 3 antes de que se produjese la desconexión del aerogenerador por exceso de velocidad de viento ha sido de $26.5^{\circ}$. Además, en línea con lo indicado al comienzo del apartado, el ángulo de pitch mínimo registrado ha sido igual a $0^{\circ}$. En relación con la velocidad de giro de las palas, en Zhang et al. (2008) se señala que este valor se puede situar en $8 \%$ s, y en Rodriguez Amenedo et al. (2003) se afirma que varía entre $5 \%$ y $10^{\circ} / \mathrm{s}$, al igual que en Dusonchet y Telaretti (2011); Pourbeik (2012b). Sin embargo, en Thet y Saitoh (2009) se afirma que la velocidad de giro de las palas de aerogeneradores modernos es de $15^{\circ} / \mathrm{s}$. Debido a la disponibilidad de los datos registrados en la presente Tesis Doctoral, se puede considerar que la velocidad de giro máxima del ángulo de paso es de 10 \% 


\begin{tabular}{l|c|l}
\hline Símbolo & Unidad & Descripción \\
\hline$K_{p w}$ & $\mathrm{pu}$ & Ganancia proporcional de la velocidad del PI. \\
\hline$K_{i w}$ & $\mathrm{pu}$ & Ganancia integral de la velocidad del PI. \\
\hline$K_{p c}$ & $\mathrm{pu}$ & Ganancia proporcional de la potencia del PI. \\
\hline$K_{i c}$ & $\mathrm{pu}$ & Ganancia integral de la potencia del PI. \\
\hline$K_{p x}$ & $\mathrm{pu}$ & Ganancia cruzada del control de pitch. \\
\hline$T_{\theta}$ & $\mathrm{s}$ & Constante de tiempo del controlador de pitch. \\
\hline$\theta_{\max }$ & $\mathrm{o}$ & Ángulo de pitch máximo. \\
\hline$\theta_{\min }$ & $\mathrm{o}$ & Ángulo de pitch mínimo. \\
\hline$d \theta_{\max }$ & $\mathrm{o} / \mathrm{s}$ & Velocidad de pitch máxima. \\
\hline$d \theta_{\min }$ & $\mathrm{o} / \mathrm{s}$ & Velocidad de pitch mínima. \\
\hline
\end{tabular}

Tabla 4.6: Parámetros necesarios para modelar el sistema de control de pitch.

El valor que se encuentra en la bibliografía para la constante de tiempo asociada con la actuación mecánica del servomotor — equipo encargado de hacer girar a las palas sobre su propio eje-, $T_{\theta}$, es más uniforme. En Clark et al. (2010); WECC REMTF (2010); Hiskens (2012) se dan valores de $0.30 \mathrm{~s}$; mientras que en Thet y Saitoh (2009); Dusonchet y Telaretti (2011) el valor del parámetro se reduce a $0.25 \mathrm{~s}$.

En línea con los valores de parámetros que se han comentado anteriormente, algunos autores han desarrollado versiones más simplificadas del modelo de control de pitch. En Lin et al. (2009) se afirma que la parte de compensación del modelo representado en la figura 4.12, es decir, el controlador que actúa sobre el error de la potencia eléctrica, puede ser omitido dado que los valores $K_{p c}$ y $K_{i c}$ son considerablemente más reducidos que los de $K_{p w}$ y $K_{i w}$. Esta hipótesis también es asumida por Thet y Saitoh (2009); Aparicio Marín (2011). 


\subsection{Conclusiones}

El presente capítulo de la Tesis Doctoral ha puesto de manifiesto una de las necesidades que actualmente posee un elevado interés internacional dentro del ámbito de la energía eólica. Se trata del desarrollo de una nueva Norma, conocida como IEC 61400-27, dedicada a la definición de modelos genéricos — también conocidos como simplificados o estándar-, tanto de aerogeneradores (Parte 1 de la Norma), como de parques eólicos (Parte 2).

Un grupo de miembros de diferentes países comenzó a elaborar esta Norma a finales del año 2009. A finales del año 2011 la versión [CD de la Norma fue enviada a los comités nacionales para obtener sus comentarios. De hecho durante el año 2012 se están puntualizando los últimos detalles relacionados con la Parte 1, al mismo tiempo que se pretende sentar las bases de la Parte 2. De esta manera, hasta mediados del año 2013 no se espera una versión definitiva de la Parte 1 de la Norma.

En el transcurso del capítulo se ha justificado la necesidad de este tipo de modelos simplificados en el sector eléctrico, diseñados para análisis de estabilidad de los sistemas eléctricos de potencia. Debido a la mayor penetración de la energía eólica en los últimos años, es necesario una correcta integración de la misma dentro de la red eléctrica. Por lo tanto, resulta necesario disponer de modelos que, aunque no posean la precisión de los modelos detallados, sí muestren el comportamiento de este tipo de generación de energía eléctrica ante diferentes perturbaciones en la red.

De manera complementaria, se ha realizado una campaña de medidas en un parque eólico para conseguir caracterizar determinados parámetros de este tipo de modelos. El resto de parámetros requeridos por los modelos han sido obtenidos en la bibliografía.

Con todo lo anterior, se han definido varios modelos que actualmente cuentan con la aprobación de todos los miembros del Comité Técnico de la IEC 61400-27. Concretamente, el modelo aerodinámico, el mecánico y el de control de pitch han sido completamente detallados.

En el caso del modelo aerodinámico, partiendo de la complejidad del modelo detallado del coeficiente de potencia, se ha analizado la simplificación obtenida mediante la linealización del modelo para su empleo en análisis de estabilidad de los sistemas eléctricos de potencia. Asumiendo que la velocidad del viento es constante durante la simulación, se ha obtenido una expresión sencilla, dependiente de un reducido número de parámetros, para representar la potencia aerodinámica transmitida por el rotor eólico.

De manera similar, el sistema mecánico de un aerogenerador ha sido reducido a un modelo de dos masas. Además de proporcionar valores típicos de los parámetros, se ha indicado cómo se pueden obtener éstos en función de las características de la turbina eólica.

También se ha modelado la actuación de un elemento de control del ae- 
rogenerador, como es el control de pitch. El componente principal de este modelo lo representa el error en las velocidades de giro del rotor eólico; aunque también incluye otro controlador activado por el error en las potencias eléctricas generadas.

Por último, mencionar que un resumen del trabajo que se ha desarrollado en este capítulo de la Tesis Doctoral ha sido publicado recientemente en el congreso IEEE Power Electronics 83 Machines for Wind Application, celebrado en Denver, USA, Honrubia Escribano et al. (2012c). 
Capítulo 5

\title{
Conclusiones, aportaciones y trabajos futuros
}

\begin{abstract}
Resumen: Se presenta el último capítulo de la Tesis Doctoral con el objeto de reunir las conclusiones generales obtenidas en los capítulos 2, 3y 4. Además, se resumen las principales aportaciones, junto con las publicaciones derivadas, y se proponen sugerencias para trabajos futuros.
\end{abstract}

\subsection{Conclusiones generales}

El aumento progresivo de la potencia eólica instalada en los sistemas de energía eléctrica genera la necesidad de evaluar la integración de este tipo de energía desde diferentes puntos de vista.

La presente Tesis Doctoral ha analizado, desde una base fundamentalmente normativa, tres temas que actualmente cuentan con un elevado interés internacional:

- Respuesta del equipamiento eléctrico presente en centrales de generación de energía eólica ante huecos de tensión.

- Comportamiento de la tecnología LiDAR e influencia sobre la generación de energía eólica.

- Modelos simplificados de aerogeneradores.

En los siguientes apartados se sintetizan las conclusiones obtenidas en cada una de las temáticas anteriores. 


\subsubsection{Equipamiento eléctrico y huecos de tensión}

Dentro de esta temática, directamente relacionada con la calidad de suministro, se ha analizado, desde un punto de vista experimental, el comportamiento de determinado equipamiento eléctrico presente en centrales de generación de energía eólica, así como en entornos industriales, ante huecos de tensión e interrupciones del suministro. Las curvas de tolerancia obtenidas mediante el ensayo de contactores, relé de falta a tierra, variador de frecuencia, ordenador personal, y diversas cargas de iluminación, permiten deducir una serie de conclusiones.

En el caso de los elementos de maniobra conocidos como contactores, más de 40.000 ensayos llevados a cabo sobre diferentes contactores de corriente alterna, bajo un procedimiento de ensayo previamente definido, dan consistencia a las conclusiones obtenidas. Se ha comprobado que el ángulo de inicio de la onda de tensión constituye un parámetro crítico en este tipo de equipamiento. De manera adicional, se ha detectado que los contactores no poseen una respuesta única ante huecos de tensión e interrupciones, sino que existe una zona de dispersión en la que su respuesta no puede ser conocida con exactitud dado su comportamiento aleatorio en dicha región. El ensayo de contactores de similares características pero distintos fabricantes permite concluir que las curvas de tolerancia son diferentes en función del fabricante. Otro de los escenarios diseñado ha permitido observar que el tipo de carga que el contactor se encuentre alimentando influye de manera considerable sobre la respuesta del mismo ante huecos de tensión e interrupciones. Por último, también se ha comprobado que la temperatura de operación del contactor se puede considerar como un parámetro crítico, dado que su curva de tolerancia resulta modificada.

El resto de equipamiento eléctrico ensayado ha mostrado una respuesta más sencilla y predecible ante las perturbaciones de la calidad del suministro eléctrico que se han efectuado. En relación con el relé de falta a tierra, en la bibliografía científica consultada no se han encontrado trabajos similares. Los ensayos realizados muestran que el comportamiento de este tipo de equipamiento ante huecos de tensión e interrupciones responde a una curva de tolerancia de morfología rectangular. Además, el ángulo de inicio de la onda de tensión no modifica dicha curva, como tampoco lo hace la temperatura de operación del equipo. Internamente, uno de los componentes principales del relé de falta a tierra es un relé de corriente continua; sin embargo, se ha comprobado que su curva de tolerancia se encuentra caracterizada por la fuente de alimentación que suministra energía a dicho relé de corriente continua.

Los ensayos realizados sobre el variador de frecuencia han permitido comprobar que, además de la forma rectangular de la curva de tolerancia, la frecuencia de salida del equipo influye sobre el tiempo que puede soportar ante una interrupción del suministro. Este fenómeno se encuentra directamente 
relacionado con el límite de sobreintensidad definido por el fabricante del equipo. También, las medidas efectuadas sobre la velocidad de giro del motor acoplado al variador de frecuencia muestran que este parámetro resulta crítico en ciertas aplicaciones como, por ejemplo, en el sistema de control de pitch de un aerogenerador. El ordenador personal ensayado muestra una respuesta similar a la del variador de frecuencia, debido a que la fuente de alimentación del $\underline{\mathrm{PC}}$ posee una característica constructiva similar a la de un variador de frecuencia.

Para finalizar el estudio de la respuesta de diverso equipamiento eléctrico ante huecos de tensión e interrupciones, las diferentes tecnologías de iluminación ensayadas permiten observar que cada una de ellas tiene un comportamiento considerablemente diferente. Cuando son alimentadas a través de un balasto electrónico en lugar de electromagnético, su sensibilidad mejora de manera relevante. Además, algunas cargas de iluminación presentan una característica adicional, que es el tiempo de reencendido necesario tras una desconexión involuntaria; lo que puede ocasionar importantes problemas en ciertas aplicaciones.

\subsubsection{LiDAR y generación eólica}

Un tema de interés actual dentro del sector eólico es la actualización de la Norma IEC 61400-12-1, relativa a la caracterización de la curva de potencia de aerogeneradores. Esta actualización está orientada a la incorporación, tanto de nuevas técnicas de medida de la velocidad y dirección del viento basadas en tecnologías remotas, así como de aspectos relacionados con el gradiente de viento. Por lo tanto, en la presente Tesis Doctoral, mediante el empleo de un equipo LiDAR se han realizado tres campañas de medidas en diversos parques eólicos de España, evaluando su comportamiento frente a las soluciones tradicionales para la medición del recurso eólico, y analizando la influencia del perfil de viento sobre la generación de energía eólica. Es importante tener en cuenta que el análisis ha sido llevado a cabo sobre terrenos de orografía compleja.

Dado que este tipo de equipos permiten medir la velocidad y dirección del viento desde $40 \mathrm{~m}$ hasta una altura de $200 \mathrm{~m}$ sobre el terreno, se han registrado una gran variedad de perfiles de viento. Éstos varían en función de la hora del día debido a la estabilidad atmosférica.

Los ensayos han permitido comprobar que el equipo LiDAR posee un elevado grado de correlación frente al tradicional anemómetro de cazoletas, tanto en la velocidad como en la dirección del viento. En relación con la velocidad del viento, se ha señalado que, por un lado, velocidades de viento mayores implican un menor error en la medida efectuada por este sistema y, por otro, que el error cometido aumenta con la altura donde se realiza la medida. Respecto a la medida de la dirección del viento, se ha detectado un error constante en todo el periodo de registro, que ha sido causado por un 
fallo humano debido a una alineación del equipo LiDAR no adecuada con respecto al norte.

El empleo de velocidades de viento equivalentes, que tienen en cuenta todo el perfil de viento situado delante del rotor del aerogenerador, ha servido para observar el efecto de suavizado de la curva de potencia para velocidades de viento próximas a las de corte. En esta línea, ha sido demostrada la necesidad de que el rotor se divida en áreas idénticas para la obtención de la velocidad equivalente. En relación con la influencia del gradiente de viento en la generación de energía, se ha mostrado que un mayor coeficiente de cortadura implica una generación de energía superior que la que se obtiene cuando existen perfiles de viento planos.

Por lo tanto, teniendo en cuenta el análisis realizado, resulta viable una reducción de la incertidumbre en la caracterización de la curva de potencia de aerogeneradores si la medida de la velocidad del viento se realiza en toda el área barrida del rotor y no se limita solamente a la medida de la velocidad del viento a la altura de buje. Además, dada la correcta correlación entre los datos registrados por sistemas LiDAR y por sistemas convencionales, se puede emplear este tipo de sistemas para realizar medidas sobre todo el rotor eólico. En este ámbito, hay que señalar que un aspecto a tener en cuenta es la disponibilidad de este tipo de sistemas; ya que resulta proporcionalmente reducida en función de la altura.

\subsubsection{Modelos simplificados de aerogeneradores}

Hoy en día, debido a la elevada penetración de energía eólica en las redes, resulta completamente necesario una correcta integración de este tipo de energía dentro de la red eléctrica para prevenir problemas de estabilidad asociados con los sistemas eléctricos. Con este interés, actualmente se está desarrollando una nueva Norma, la IEC 61400-27, cuyo Comité Técnico se formó a finales del año 2009, que persigue la implementación de modelos genéricos — también conocidos como simplificados o estándar-, tanto de aerogeneradores como de parques eólicos, que permitan mostrar el comportamiento de este tipo de centrales de generación de energía eléctrica ante diferentes perturbaciones en la red de suministro.

Dado que la IEC 61400-27 es una Norma reciente de nueva creación, aún no se cuenta con la definición de todos los modelos que la componen. Con ello, en la presente Tesis Doctoral se han definido y revisado algunos de los modelos que actualmente cuentan con la aprobación de todos los miembros del Comité Técnico.

Se ha mostrado el modelo aerodinámico linealizado que, asumiendo que la velocidad del viento es constante durante la simulación, permite reflejar la respuesta del aerogenerador en estudios de estabilidad de los sistemas eléctricos de potencia, mediante el empleo de un reducido número de parámetros. 
Con el mismo interés, el sistema mecánico de un aerogenerador ha sido reducido a un modelo de dos masas. Por último, también se ha modelado un sistema de control del aerogenerador, como es el sistema de control de pitch.

Todo el trabajo desarrollado dentro de esta temática también ha incluido la caracterización de determinados parámetros de este tipo de modelos. La parametrización de algunos parámetros ha sido llevada a cabo mediante analizadores trifásicos de calidad de la energía instalados en un parque eólico, así como a raíz del sistema SCADA de dicho parque. El resto de parámetros requeridos por los modelos han sido obtenidos en la bibliografía.

\subsection{Aportaciones}

Las principales aportaciones novedosas y originales de la presente Tesis Doctoral se pueden resumir en las siguientes líneas:

- Evaluación de la respuesta de diverso equipamiento eléctrico presente en centrales de generación de energía eólica ante determinadas perturbaciones de la red de suministro.

- Caracterización de los sistemas LiDAR para la evaluación del recurso eólico.

- Evaluación del sistema LiDAR frente a sistemas tradicionales para la medición de la velocidad y dirección del viento.

- Análisis de la influencia del gradiente de viento en la generación eólica.

- Desarrollo de determinados modelos simplificados de aerogeneradores.

En línea con el trabajo desarrollado se han realizado las siguientes publicaciones:

- Honrubia Escribano, Andrés; Gómez Lázaro, Emilio; Molina García, Ángel; Fuentes Moreno, Juan Álvaro. "Influence of Voltage Dips on Industrial Equipment: Analysis and Assessment". International Journal of Electrical Power 63 Energy Systems. Año: 2012. No de páginas: 9.

- Honrubia Escribano, Andrés; Gómez Lázaro, Emilio; Molina García, Ángel; Vigueras Rodríguez, Antonio. "Sistemas de Evaluación del Recurso Eólico: Integración de Nuevas Soluciones Basadas en Tecnología Láser". DYNA Ingeniería e Industria. Año: 2012. No de páginas: 9.

- Honrubia Escribano, Andrés; Vigueras Rodríguez, Antonio; Gómez Lázaro, Emilio. "The influence of turbulence and vertical wind profile in wind turbine power curve". Progress in Turbulence and Wind Energy IV. Editorial: Springer, vol. 141, páginas 251 - 254, 2012. 
Así como contribuciones a congresos:

- Honrubia Escribano, Andrés; Gómez Lázaro, Emilio; Vigueras Rodriguez, Antonio; Molina García, Ángel; Fuentes Moreno, Juan Álvaro; Muljadi, Eduard. "Assessment of DFIG Simplified Model Parameters Using Field Test Data". IEEE Symposium on Power Electronics \& $M a$ chines for Wind Application. Denver, USA, 2012.

- Honrubia Escribano, Andrés; Pujante López, Antonio Jesús; Vigueras Rodríguez, Antonio; Gómez Lázaro, Emilio; López, Manuel; Jiménez, Raúl; Martínez, Manuel. "Comparison of wind speed measurements over complex terrain using a LIDAR system". European Wind Energy Conference and Exhibition. Brussels, Belgium, 2011.

- Honrubia Escribano, Andrés; Vigueras Rodríguez, Antonio; Gómez Lázaro, Emilio. "The influence of turbulence and vertical wind profile in wind turbine power curve". Conference on Turbulence. Bertinoro, Italy, 2010 .

- Honrubia Escribano, Andrés; Gómez Lázaro, Emilio; Jiménez Moreno, Graciano; Molina García, Ángel. "Analysis of the AC-Contactor Electrical Behaviour under Voltage Dips". IEEE XIX International Conference on Electrical Machines. Rome, Italy, 2010.

- Honrubia Escribano, Andrés; Vigueras Rodríguez, Antonio; Gómez Lázaro, Emilio. "Vertical wind profile measurement using a pulsed LIDAR system". International Symposium for the Advancement of Boundary Layer Remote Sensing. Paris, France, 2010.

- Honrubia Escribano, Andrés; Vigueras Rodríguez, Antonio; Gómez Lázaro, Emilio. "Comparative analysis between LIDAR technologies and common wind speed meters". World Wind Energy Conference. Istanbul, Turkey, 2010.

- Honrubia Escribano, Andrés; Vigueras Rodríguez, Antonio; Gómez Lázaro, Emilio; Rodríguez Sánchez, David. "The influence of wind shear in wind turbine power estimation". European Wind Energy Conference. Warsaw, Poland, 2010.

\subsection{Trabajos futuros}

En virtud de las conclusiones obtenidas en la Tesis Doctoral, se pueden sugerir algunas líneas de investigación:

- Estudio de la mejora de la respuesta de determinada aparamenta eléctrica ante perturbaciones en la red de suministro; tanto desde el punto de vista de la simulación de modelos como del experimental. 
- Empleo de un sistema LiDAR durante una campaña de medida no inferior a un año de manera continua. De esta manera se conseguiría obtener un verdadero feedback de los requerimientos de este tipo de equipos remotos, al mismo tiempo que, junto con un sistema SCADA, se podría evaluar la generación eólica en función de un elevado número de perfiles de viento registrados.

- Filtrado de información registrada por sistemas LiDAR.

- Desarrollo e implementación de modelos genéricos completos de aerogeneradores y parques eólicos.

- Validación de modelos simplificados de aerogeneradores con respecto a modelos detallados de fabricantes y medidas registradas en parques eólicos. 



\section{Bibliografía}

Abu-Rub, H., Holtz, J., Rodriguez, J. y Baoming, G. Medium-voltage multilevel converters-State of the art, challenges, and requirements in industrial applications. IEEE Transactions on Industrial Electronics, vol. 57, no. 8, páginas $2581-2596,2010$.

Ackerman, T. Et Al. Wind Power in Power Systems. John Wiley, 2005.

AEE. Eólica 12. Informe técnico, Asociación Empresarial Eólica, 2012.

Akolkar, S. y Kushare, B. Effect of point on wave angle on sensitivity of AC coil contactor and SMPS to voltage sags. IPEC Conference Proceedings, páginas 957 - 961, 2010.

Albers, A. Critical limitations of wind turbine power curve warranties. European Wind Energy Association, 2012.

Albers, A., Franke, K., Wagner, R., Courtney, M. y Boquet, M. Ground-based remote sensor uncertainty-a case estudy for a wind lidar. European Wind Energy Association, 2012.

Albers, A., Janssen, A. W. y Mander, J. Comparison of lidars, german test station for remote wind sensing devices. Informe técnico, Deutsche Windguard Consulting GmbH, 2008.

Antoniou, I., Courtney, M., Jørgensen, H. E., Mikkelsen, T., Hunerbein, S. V., Bradley, S., Piper, B., Harris, M., Marti, I., ArisTU, M., Foussekis, D. y Nielsen, M. P. Remote sensing the wind using lidars and sodars. European Wind Energy Conference, 2007a.

Antoniou, I., Jørgensen, H. E., Mikkelsen, T., Frandsen, S., Barthelmie, R., Perstrup, C. y Hurtig, M. Offshore wind profile measurements from remote sensing instruments. European Wind Energy Conference, 2006. 
Antoniou, I. y Pedersen, S. M. Influence of turbulence, wind shear and low-level jets on the power curve and the AEP of a wind turbine. European Wind Energy Conference, 2009.

Antoniou, I., Wagner, R., Pedersen, S. M., Paulsen, U., Madsen, H. A., Jorgensen, H. E., Thomsen, K., Enevoldsen, P. y ThesbJERG, L. Influence of wind characteristics on turbine performance. European Wind Energy Conference, 2007b.

APARICIO MARÍN, N. Nuevas estrategias para la contribución de los parques eólicos al control de frecuencia de los sistemas eléctricos. Tesis Doctoral, Universidad Politécnica de Valencia, 2011.

Asmine, M., Brochu, J., Fortmann, J., Gagnon, R., Kazachkov, Y., Langlois, C. E., Larose, C., Muljadi, E., MacDowell, J., PourBeik, P., Seman, S. y Wiens, K. Model validation for wind turbine generator models. IEEE Transactions on Power Systems, vol. 26(3), páginas $1769-1782,2011$. ISSN 0885-8950.

Baggini, A. Handbook of Power Quality. John Wiley \& Sons, 2008.

Baran, M., Maclaga, J., Kelley, A. y Craven, K. Effects of power disturbances on computer systems. IEEE Transactions on Power Delivery, vol. 13, no. 4, páginas 1309 - 1315, 1998.

BARros, J. y DiEGO, R. Effects of nonsinusoidal supply on voltage tolerance of equipment. IEEE Power Engineering Review, vol. 22, no. 7, páginas 46 $-47,2002$.

Barros, J. y Diego, R. Discussion of "sensitivity of personal computers to voltage sags and short interruptions". IEEE Transactions on Power Delivery, vol. 21, no. 1, páginas 543 - 544, 2006.

Behnke, M., Ellis, A., Kazachkov, Y., McCoy, T., Muluadi, E., Price, W. y SAnchez-Gasca, J. Development and validation of WECC variable speed wind turbine dynamic models for grid integration studies. AWEA WindPower Conference, 2007.

Belchior, F., Oliviera, J., Leao, P. y Souto, O. Ride-through alternatives for adjustable-speed drives - computational analysis. International Symposium on Industrial Electronics, vol. 2, páginas 1032 - 1036, 2003.

Bleiber, K., Kramkowski, T., Cosack, N. y Braun, K. The influence of meteorological parameters on the operational behavior of multimegawatt WEC. German Wind Energy Conference, 2006.

Bo, Y., Xinping, D., Yan, L., XiA, L. y Hong, L. Effect of voltage sags on the Z-source adjustable-speed drives. 30th Chinese Control Conference, páginas 3517 - 3522, 2011. 
Bok, J., Drapela, J. y Toman, P. Personal computers immunity to short voltage dips and interruptions. 13th International Conference on Harmonics and Quality of Power, páginas 1-6, 2008.

BoldeA, I. Control issues in adjustable speed drives. IEEE Industrial Electronics Magazine, vol. 2, no. 3, páginas 32 - 50, 2008.

Bollen, M. Understanding power quality: Voltage sags and interruptions. IEEE Press, New York, 2000.

Bollen, M., Stephens, M., Djokic, S., Stockman, K., Brumsickle, B., Milanovic, J., Romero Gordón, J., Neumann, R., Ethier, G., Córcoles, F., Ferguson, A., Goossens, P., Ligot, P., Lopes Leiria, A., Marteyn, P., McEachern, A., Mentzer, J., McMichael, I., Minnaar, U., Reusel, K. y Zavoda, F. Voltage dip immunity of equipment and installations. Informe técnico, CIGRE, 2010.

Bollen, M. y Zhang, L. Analysis of voltage tolerance of AC adjustablespeed drives for three-phase balanced and unbalanced sags. IEEE Transactions on Industry Applications, vol. 36, no. 3(3), páginas 904 - 910, 2000 .

Bollen, M. H. J. y ZhAng, L. D. Different methods for classification of three-phase unbalanced voltage dips due to faults. Electric Power Systems Research, vol. 66, páginas 59-69, 2003.

BRAÑA, I. Turbine-Mounted Lidar: The pulsed lidar as a reliable alternative. Proyecto Fin de Carrera, Gotland University, 2011.

Brauner, G. y Hennerbichler, C. Voltage dips and sensitivity of consumers in low voltage networks. 16th International Conference and Exhibition on Electricity Distribution, vol. 2, 2001.

Brown, R. E. Electric Power Distribution Reliability. CRC Press, 2009.

Burnham, D., Santoso, S. y Muluadi, E. Variable rotor-resistance control of wind turbine generators. IEEE Power Energy Society General Meeting, 2009.

Cañadillas, B., Westerhellweg, A. y Neumann, T. Testing the performance of a ground-based wind LiDAR system. DEWI Magazin, vol. 38, páginas $58-64,2011$.

Carrillo, C., Vidal-Vilarino, F., Suárez-Álvarez, M., Saez-Tort, M. y Díaz-Dorado, E. Impact of ASD settings in its LVRT behaviour. International Symposium on Power Electronics Electrical Drives Automation and Motion, páginas 339 - 342, 2010. 
Chieh-Tsung, C. Dynamic stability analysis based on energy-passivity considerations. WSEAS TRANSACTIONS on CIRCUITS AND SYSTEMS, vol. 7, páginas $119-128,2008$.

Chin-Yu, H. y Chieh-Tsung, C. A new compensation strategy for an $\mathrm{AC}$ contactor under voltage-sag events. IEEE Conference on Robotics Automation and Mechatronics, páginas 52 - 57, 2010.

Chilukuri, M. V., Lee, M. Y. y Phang, Y. Y. Voltage sag sensitivity of home appliances and office equipment. 20th International Conference and Exhibition on Electricity Distribution, páginas 1 - 6, 2009.

CHuCK, T. Overview of SEMI F47-0706. SEMICON, 2006.

Clark, K., Miller, N. W. y Sánchez-Gasca, J. J. Modeling of ge wind turbine-generators for grid studies. Informe Técnico 4.5, General Electric International, Inc., Schenectady, New York, 2010.

CNE. Información estadística sobre las ventas de energía del régimen especial. Informe técnico, Comisión Nacional de la Energía, 2012.

Collins, E. y Zapardiel, F. An experimental assessment of AC contactor behavior during voltage sags. IEEE International Symposium on Industrial Electronics (ISIE), vol. 2, páginas 439 - 444, 1997.

Collins, J., E.R. y BRIDGwood, M. The impact of power system disturbances on AC-coil contactors. IEEE Annual Textile, Fiber, and Film Industry Technical Conference, 1997.

Comech Moreno, M. P. Análisis y ensayo de sistemas eólicos ante huecos de tensión. Tesis Doctoral, Universidad de Zaragoza, Zaragoza (Spain), 2007.

Cornick, K. y Li, H. Power quality and voltage dips: problem, requirements, responsibilities. International Conference on Advances in Power System Control, Operation and Management., vol. 1, páginas 149 - 156, 2000 .

Costa, A., Rodrigues, D., de Freitas, L., Vieira, J., Coelho, E., Farias, V. y Freitas, L. Hybrid three-phase rectifier with high power factor and voltage sags ride-through capability for utility interface of adjustable speed drives. Brazilian Power Electronics Conference, páginas $582-588,2011$.

Costello, P. The case for solid-state switching. IET Computer $\&$ Control Engineering, vol. 18, páginas 14-15, 2007. 
Counihan, J. Adiabatic atmospheric boundary layers: A review and analysis of data from the period 1880-1972. Atmospheric Environment, vol. 9, no. 10, páginas $871-905,1975$.

Courtney, M. Measurements. Informe técnico, Summer School of the European Academy of Wind Energy, 2010.

Courtney, M., Wagner, R. y Lindelow, P. Commercial lidar profilers for wind energy. a comparative guide. European Wind Energy Conference, 2008a.

Courtney, M., Wagner, R. y Lindelow, P. Testing and comparison of lidars for profile and turbulence measurements in wind energy. 14th International Symposium for the Advancement of Boundary Layer Remote Sensing, 2008b.

Coyle, T. Performance testing low-voltage ground-fault protection. IEEE Transactions on Industry Applications, vol. 38, no. 4, páginas 981 - 989, 2002.

David, A., Lajoie-Mazenc, E. y Sol, C. Ride-through capability of AC adjustable speed drives in regards to voltage dips on the distribution network. Fifth European Conference on Power Electronics and Applications, vol. 6, páginas 139 - 144, 1993.

Deswal, S., Dahiya, R. y Jain, D. Ride-through capability of adjustablespeed drive during various power quality events using supercapacitor. IEEE Electrical Power Energy Conference (EPEC), páginas 1 - 6, 2009.

Deswal, S., Dahiya, R. y Jain, D. Ride-through alternatives of adjustable speed drives (ASD's) during fault conditions. Joint International Conference on Power Electronics, Drives and Energy Systems, páginas 1 $-7,2010$.

Deswal, S., Dahiya, R. y Jain, D. Investigations on ride-through capabilities of asd's during three-phase unsymmetrical faults. 37th Annual Conference on IEEE Industrial Electronics Society, páginas 1915 - 1921, 2011.

Diaz, F., Azcondo, F., Ortiz, F., Ortiz, A., Manna, M. y Renedo, C. Effects of voltage sags on different types of ballasts for 150-W HPS lamps. 9th International Conference on Electrical Power Quality and Utilisation, páginas $1-6,2007$.

Directiva 2005/32/CE. DEL PARLAMENTO EUROPEO Y DEL CONSE$J O$, por la que se instaura un marco para el establecimiento de requisitos de diseño ecológico aplicables a los productos que utilizan energía, 2005. 
Directiva 2009/125/CE. DEL PARLAMENTO EUROPEO Y DEL CONSE$J O$, por la que se instaura un marco para el establecimiento de requisitos de diseño ecológico aplicables a los productos relacionados con la energía, 2009.

Djokic, S., Desmet, J., Vanalme, G., Milanovic, J. y Stockman, K. Sensitivity of personal computers to voltage sags and short interruptions. IEEE Transactions on Power Delivery, vol. 20, no. 1, páginas 375 - 383, $2005 \mathrm{a}$.

DJokic, S. y Milanovic, J. Closure on "sensitivity of personal computers to voltage sags and short interruptions". IEEE Transactions on Power Delivery, vol. 21, no. 1, páginas $544-545,2006$.

Djokic, S., Milanovic, J. y Kirschen, D. Sensitivity of AC coil contactors to voltage sags, short interruptions, and undervoltage transients. IEEE Transactions on Power Delivery, vol. 19, no. 3, páginas 1299 - 1307, 2004.

Djokic, S., Munshi, S. y Cresswell, C. The influence of overcurrent and undervoltage protection settings on ASD sensitivity to voltage sags and short interruptions. 4th IET Conference on Power Electronics, Machines and Drives, páginas 130 - 134, 2008.

Djokic, S., Stockman, K., Milanovic, J., Desmet, J. y Belmans, R. Sensitivity of AC adjustable speed drives to voltage sags and short interruptions. IEEE Transactions on Power Delivery, vol. 20, no. 1, páginas $494-505,2005 b$.

Dorr, D., Mansoor, A., Morinec, A. y Worley, J. Effects of power line voltage variations on different types of 400-W high-pressure sodium ballasts. IEEE Transactions on Industry Applications, vol. 23, no. 2, páginas $472-476,1997$ a.

Dorr, D. S., Hughes, M. B., Gruzs, T. M., Jurewicz, R. E. y McClaine, J. L. Interpreting recent power quality surveys to define the electrical environment. IEEE Transactions on Industry Applications, vol. 33, 1997b.

Drapela, J., Bok, J., Slezingr, J. y Pithart, J. Light sources immunity to short voltage dips and interruptions. 20th International Conference and Exhibition on Electricity Distribution, páginas 1 - 4, 2009.

Duran-Gomez, J., Enjeti, P. y Woo, B. O. Effect of voltage sags on adjustable-speed drives: a critical evaluation and an approach to improve performance. IEEE Transactions on Industry Applications, vol. 35, no. 6, páginas $1440-1449,1999$. 
DurÁn, L. y BARrios, A. Uso de la técnica SODAR para la evaluación del recurso eólico. XXIX Jornadas Científicas de la Asociación Meteorológica Española, 2006.

Dusonchet, L. y Telaretti, E. Effects of electrical and mechanical parameters on the transient voltage stability of a fixed speed wind turbine. Electric Power Systems Research, vol. 81, no. 7, páginas 1308 - 1316, 2011.

Dwivedi, U., Shakya, D. y Singh, S. Power quality monitoring and analysis: An overview and key issues. International Journal of Signal System Control and Engineering Application, vol. 1, no. 1, páginas 74 88, 2008.

ELCF. Make the switch. The ELC Road Map for Deploying energy efficient lighting technology across Europe. Informe técnico, European Lamp Companies Federation, 2007.

Ellis, A., Kazachkov, Y., Muljadi, E., Pourbeik, P. y SanchezGASCA, J. Description and technical specifications for generic WTG models - A status report. IEEE/PES Power Systems Conference and Exposition (PSCE), páginas 1-8, 2011a.

Ellis, A., Muljadi, E., Sanchez-Gasca, J. y Kazachkov, Y. Generic models for simulation of wind power plants in bulk system planning studies. IEEE Power and Energy Society General Meeting, páginas $1-8$, $2011 b$.

EmeIs, S. y TURK, M. Comparison of logarithmic wind profiles and power law wind profiles and their applicability for offshore wind profiles. En Wind Energy. Proceeding of the Euromech Colloquium. 2007.

Endrejat, F., VAn Blerk, B. y Vignolo, G. Experience with new large adjustable speed drive technology for multiple synchronous motors. Petroleum and Chemical Industry Conference Europe, páginas 1 - 10, 2008.

EPRI. Performance of a hold-in device for relays, contactors and motor starters. Informe técnico, Electric Power Research Institute, 1998.

Espinosa, A., Ruiz, J.-R., Cusido, J. y Morera, X. Sensorless control and fault diagnosis of electromechanical contactors. IEEE Transactions on Industrial Electronics, vol. 55, no. 10, páginas 3742 - 3750, 2008.

Esteban PéRez, M. D. Propuesta de una metodología para la implantación de parques eólicos offshore. Tesis Doctoral, Universidad Politécnica de Madrid, 2009.

EWEA. Wind energy - the facts. Informe técnico, European Wind Energy Association, 2009. 
Fernandez, A., Sebastian, J., Hernando, M., Villegas, P. y GarCIA, J. Helpful hints to select a power-factor-correction solution for lowand medium-power single-phase power supplies. IEEE Transactions on Industrial Electronics, vol. 52, no. 1, páginas 46 - 55, 2005.

Firtin, E., Güler, O. y AKDaG, S. A. Investigation of wind shear coefficients and their effect on electrical energy generation. Applied Energy, vol. 88, no. 11, páginas 4097 - 4105, 2011.

Flores-Arias, J., Moreno-Munoz, A., De la Rosa, J., Gil-de Castro, A., Pallares-Lopez, V. y Moreno-Garcia, I. Electromagnetic compatibility test system. 7th International Conference-Workshop on Compatibility and Power Electronics (CPE), páginas 62 - 67, 2011.

Fortmann, J. Generic aerodynamic model for simulation of variable speed wind turbines. 9th International Workshop on Large-Scale Integration of Wind Power into Power Systems as well as on Transmission Networks for Offshore Wind Power Plants, 2010.

Fortmann, J., Engelhardt, S., Kretschmann, J., Janben, M., Neumann, T. y ERlich, I. Generic simulation model for dfg and full size converter based wind turbines. 9th International Workshop on Large-Scale Integration of Wind Power into Power Systems as well as on Transmission Networks for Offshore Wind Farms, 2010.

FousseKis, D. Investigating wind flow properties in complex terrain using 3 lidars and a meteorological mast. European Wind Energy Conference, 2009 .

Foussekis, D., Mouzakis, F., Papadopoulos, P. y Vionis, P. Wind profile measurements using a lidar and a $100 \mathrm{~m}$ mast. European Wind Energy Conference, 2007.

Foussekis, D., Stefanatos, N. y Mouzakis, F. Operation of the windcube v2 lidar at CRES test station. Informe técnico, Centre for Renewable Energy Sources, 2011.

Franchini, S. N. Fuentes de incertidumbre en anemometría sónica. Tesis Doctoral, Universidad Politécnica de Madrid, 2006.

Frater, L. y WATSON, N. Light flicker sensitivity of high efficiency compact fluorescent lamps. Australasian Universities Power Engineering Conference, páginas $1-6,2007$.

Freris, L. L. Wind energy conversion systems. Prentice Hall, 1990.

Fuji, T. y Fukuchi, T. Laser Remote Sensing. CRC Taylor \& Francis, 2005. 
Gencer, O., Ozturk, S. y Erfidan, T. A new approach to voltage sag detection based on wavelet transform. International Journal of Electrical Power 63 Energy Systems, vol. 32, no. 2, páginas 133 - 140, 2010.

GiPe, P. Wind Power: Renewable Energy for Home, Farm, and Business. Chelsea Green Publishing, 2004.

GonzÁlez Rodríguez, A. G. Improvement of a fixed-speed wind turbine soft-starter based on a sliding-mode controller. Tesis Doctoral, Universidad de Sevilla, 2006.

Gottschall, J. y Courtney, M. Verification test for three WindCube WLS7 LiDARs at the høvsøre test site. Informe técnico, Technical University of Denmark, 2010.

Gottschall, J., Courtney, M. S., Wagner, R., Jørgensen, H. E. y Antoniou, I. Lidar profilers in the context of wind energy-a verification procedure for traceable measurements. Wind Energy, vol. 15, no. 1, páginas 147 - 159, 2012.

Gross, G., Frey, T. y Trute, P. On the applicability of numerical models for calculating local winds in complex terrain. DEWI Magazin, vol. 20, no. 2,2002 .

GWEC. Global wind report. Annual market update 2011. Informe técnico, Global Wind Energy Council, 2012.

Hannan, M., Nordin, N. y Mohamed, A. Analysis of AC contactor model during voltage sag with point-in-wave of initiation. Przeglad Elektroteckniczny (Electrical Review), vol. 6, páginas 351 - 353, 2012.

Hansen, O. F., Hansen, S. O. y Kristensen, L. Wind tunnel calibration of cup anemometers. AWEA WindPower Conference, 2012.

HARDi, S. y DAut, I. Sensitivity of low voltage consumer equipment to voltage sags. 4th International Power Engineering and Optimization Conference, páginas 396 - 401, 2010.

Hau, E. Wind Turbines: Fundamentals, Technologies, Application, Economics. Springer, 2nd edición, 2005. ISBN 3540242406.

Heisselmann, H., Hölling, M. y Peinke, J. The sphere anemometer - a fast alternative to cup anemometry. Progress in Turbulence III, vol. 131, páginas $69-72,2009$.

Hiskens, I. Dynamics of type-3 wind turbine generator models. IEEE Transactions on Power Systems, vol. 27, no. 1, páginas 465 - 474, 2012. 
Honrubia, A., Pujante-López, A. J., Vigueras-Rodríguez, A., Gómez-Lázaro, E., López, M., Jiménez, R. y Martínez, M. Comparison of wind speed measurements over complex terrain using a lidar system. European Wind Energy Conference, 2011.

Honrubia, A., Vigueras, A., Gomez, E., Mejías, M. y Lainez, I. Comparative analysis between lidar technologies and common wind speed meters. World Wind Energy Conference, 2010a.

Honrubia, A., Vigueras-Rodríguez, A. y Gómez-Lazaro, E. The influence of turbulence and vertical wind profile in wind turbine power curve. iTi Conference on Turbulence, 2010b.

Honrubia, A., Vigueras-Rodríguez, A. y Gómez-Lazaro, E. The influence of turbulence and vertical wind profile in wind turbine power curve. Progress in Turbulence and Wind Energy IV, vol. 141, páginas 251 $-254,2012$.

Honrubia, A., Vigueras-Rodríguez, A. y Gómez-Lázaro, E. Vertical wind profile measurement using a pulsed lidar system. 15th International Symposium for the Advancement of Boundary Layer Remote Sensing, 2010c.

Honrubia, A., Vigueras-Rodríguez, A., Gómez-Lázaro, E. y Rodríguez-SÁnchez, D. The influence of wind shear in wind turbine power estimation. European Wind Energy Conference, 2010d.

Honrubia-Escribano, A., Gómez-LáZaro, E., Molina-García, A. y Fuentes, J. Influence of voltage dips on industrial equipment: Analysis and assessment. International Journal of Electrical Power 83 Energy Systems, vol. 41, no. 1, páginas 87 - 95, 2012a.

Honrubia-Escribano, A., Gómez-Lázaro, E., Molina-García, A., Fuentes, J. A. y Muljadi, E. A survey of voltage dips in photovoltaic plants. IEEE PES General Meeting, páginas 1-6, 2012b.

Honrubia Escribano, A., Gómez-Lázaro, E., Molina-García, A. y Vigueras-Rodríguez, A. Sistemas de evaluación del recurso eólico: Integración de nuevas soluciones basadas en tecnología láser. DYNA Ingeniería e Industria, vol. 87, no. 5, páginas 540 - 548, 2012a.

Honrubia Escribano, A., Gómez-Lázaro, E., Molina-García, A., Vigueras-Rodríguez, A. y GonzÁlez-SÁnchez, B. Assessment of a novel energy efficient system for fluorescent lighting installations. Przeglad Elektrotechniczny (Electrical Review), vol. 88, no. 1a, páginas 100 - 103, 2012b. 
Honrubia Escribano, A., Gómez-Lázaro, E., Vigueras-Rodríguez, A., Molina-García, A., Fuentes, J. A. y Muljadi, E. Assessment of DFIG simplified model parameters using field test data. IEEE Symposium on Power Electronics 83 Machines for Wind Application, páginas 1 - 7, 2012c.

Honrubia Escribano, A., Gómez-Lázaro, E., Jiménez-Moreno, G. y Molina-García, A. Analysis of the AC-Contactor Electrical Behavior under Voltage Dips. XIX International Conference on Electrical Machines, 2010.

HsieH, J.-C. y Lin, J. Novel single-stage self-oscillating dimmable electronic ballast with high power factor correction. IEEE Transactions on Industrial Electronics, vol. 58, no. 1, páginas 250 - 262, 2011.

IEEE Std. 1159-2009. IEEE Recommended Practice for Monitoring Electric Power Quality. 2009.

IEEE Std. 1250-2011. IEEE Guide for identifying and improving voltage quality in power systems. 2011.

International Electrotechnical Commission. IEC 61400-12-1: Power performance measurements of electricity producing wind turbines. 2005.

International Electrotechnical Commission. IEC 61000-4-30 ed. 2.0: Test and measurement techniques. methods for measuring the quality of supply - electromagnetic compatibility (EMC). 2008.

International Electrotechnical Commission. IEC 61400-27-1: Electrical simulation models for wind power generation - wind turbines. CD version.

International Electrotechnical Commission. IEC 61400-27-2: Electrical simulation models for wind power generation - wind power plants. NWIP version.

Ismail, N., Kasim, L., Mokhtar, N., YaAkub, T. y Mokhlis, H. Digital analysis and development of ride-through capability of AC contactor with respect to voltage sag characteristics. The 2nd International Conference on Computer and Automation Engineering, vol. 5, páginas 177 - 181, 2010.

ITI. ITI (CBEMA) Curve Application Note. Information Technology Industry Council, Washington, DC, 2000.

Iyoda, I., Hirata, M., Shigei, N., Pounyakhet, S., Ota, K. y Ise, T. Affect of voltage sags on electro-magnetic contactor. 9th International Conference on Electrical Power Quality and Utilisation, páginas 1 - 6, 2007. 
Jaynes, D. W., Manwell, J. F., McGowan, J. G., Stein, W. M. y Rogers, A. L. MTC final progress report: LIDAR. Informe técnico, Renewable Energy Research Laboratory, 2007.

JeOng, S., LeE, G. y Gim, J. The study on the characteristics of operating limits of ac contactor during voltage sag. IEEE Transmission Distribution Conference Exposition: Asia and Pacific, 2009.

Jiménez Buendía, F. y Barrasa Gordo, B. Generic simplified simulation model for DFIG with active crowbar. 11th International Workshop on Large-Scale Integration of Wind Power into Power Systems as well as on Transmission Networks for Offshore Wind Power Plants, 2012.

Johansson, T. B. y Burnham, L. Renewable Energy: Sources For Fuels And Electricity. Island Press, 1992.

Justus, C. G. Winds and wind system performance. Franklin Institute Press, 1978.

Kennedy, J. M., Fox, B., Littler, T. y Flynn, D. Validation of fixed speed induction generator models for inertial response using wind farm measurements. IEEE Transacions on Power Systems, vol. 26(3), páginas 1454-1461, 2011. ISSN 0885-8950.

Keung, P.-K., Kazachkov, Y. y Senthil, J. Generic models of wind turbines for power system stability studies. International Conference on Advances in Power System Control, Operation and Management, páginas $1-6,2009$.

Keus, A., Abrahams, R., Van Coller, J. y Koch, R. Analysis of voltage dip (sag) testing results of a $15 \mathrm{~kW} \mathrm{PWM} \mathrm{adjustable} \mathrm{speed} \mathrm{drive} \mathrm{(ASD).}$ International Conference on Electric Machines and Drives, páginas 213 $215,1999$.

Kingrey, L., Painter, R. y Locker, A. Applying high-resistance neutral grounding in medium-voltage systems. IEEE Transactions on Industry Applications, vol. 47, no. 3, páginas 1220 - 1231, 2011.

Kundur, P., Paserba, J., Ajjarapu, V., Andersson, G., Bose, A., Canizares, C., Hatziargyriou, N., Hill, D., Stankovic, A., Taylor, C., Van Cutsem, T. y Vittal, V. Definition and classification of power system stability. IEEE/CIGRE joint task force on stability terms and definitions. IEEE Transactions on Power Systems, vol. 19, no. 3, páginas 1387-1401, 2004.

Kushare, B. E. y Ghatol, A. A. Investigation of cost effective method to improve voltage sag ride through capability of ac coil contactors. IET-UK 
International Conference on Information and Communication Technology in Electrical Sciences, páginas 452 - 457, 2007.

Kyei, J., Ayyanar, R., Heydt, G., Thallam, R. y Blevins, J. The design of power acceptability curves. IEEE Transactions on Power Delivery, vol. 17, no. 3, páginas $828-833,2002$.

LANG, S. y MCKeOGH, E. LIDAR and SODAR measurements of wind speed and direction in upland terrain for wind energy purposes. Remote Sensing, vol. 3, no. 9, páginas 1871 - 1901, 2011.

Lange, M. y Focken, U. Physical Approach to Short-Term Wind Power Prediction. Springer, 2005.

Langlois, C. E., Lefebvre, D., Dube, L. y Gagnon, R. Developing a Type-III wind turbine model for stability studies of the hydro-quebec network. 8th International Workshop Large-Scale Integration of Wind Power Into Power Systems, páginas 674 - 679, 2009.

Langreder, W., Kaiser, K., Hohlen, H. y Hojstrup, J. Turbulence correction for power curves. European Wind Energy Conference, 2004.

Larsen, S., Gryning, S., Jensen, N., Jørgensen, H. y Mann, J. Mean wind and turbulence in the atmospheric boundary layer above the surface layer. En Wind Energy (editado por J. Peinke, P. Schaumann y S. Barth), páginas 21 - 25. Springer, 2007.

Lin, L., Zhang, J. y YAng, Y. Comparison of pitch angle control models of wind farm for power system analysis. En IEEE Power Energy Society General Meeting. 2009. ISBN 978-1-4244-4240-9.

Locker, I. y WoOdWARD, A. Introducing the new ZephIR 300. Windtech International, 2010.

Lubitz, W. D. Effects of tower shadowing on anemometer data. 11th Americas Conference on Wind Engineering, 2009.

Makinen, A., Raipala, O., Repo, S. y Tuusa, H. Influence of DFIG reactive current injection during a voltage dip on the operation of wind turbine circuit breaker. IEEE 8th International Conference on Power Electronics and ECCE, páginas $88-95,2011$.

Manwell, J. F., McGowan, J. G. y Rogers, A. L. Wind Energy Explained: Theory, Design and Application. John Wiley \& Sons, 2009.

Mata Dumenjó, M., Sánchez Navarro, J., Casadevall Benet, V., Gil Cepeda, J. y García Caballero, L. Simplified model of DFiG. 10th International Workshop on Large-Scale Integration of Wind Power 
into Power Systems as well as on Transmission Networks for Offshore Wind Power Farms, 2011.

Mathew, S. Wind Energy: Fundamentals, Resource Analysis and Economics. Springer, 2006.

McGranaghan, M. y Roettger, B. Economic Evaluation of Power Quality. IEEE Power Engineering Review, vol. 22, no. 2, páginas 8-12, 2002.

MEASNET. ANEMOMETER CALIBRATION PROCEDURE, 2 edición, 2009a.

MEASNET. EVALUATION OF SITE-SPECIFIC WIND CONDITIONS, 1 edición, 2009b.

Menniti, D., Burgio, A. y Fedele, G. A cost effective ac voltage regulator to mitigate voltage sags and dim lamps in street-lighting applications. 9th International Conference on Environment and Electrical Engineering, páginas 396 - 399, 2010.

Mikkelsen, T. Remote sensing of wind. Informe técnico, Technical University of Denmark, 2012.

Mohamad, H. y Nor, K. Evaluation on sensitivity of ac contactor during voltage sag. IEEE Region 10 Conference TENCON, vol. 3, páginas 295 298,2004 .

Moon, J.-F., Yun, S.-Y. y KIM, J.-C. Quantitative evaluation of the impact of repetitive voltage sags on low-voltage loads. IEEE Transactions on Power Delivery, vol. 22, no. 4, páginas 2395 - 2400, 2007.

dos Santos Dias de Moraes, P. y Perin, A. An electronic control unit for reducing contact bounce in electromagnetic contactors. IEEE Transactions on Industrial Electronics, vol. 55, no. 2, páginas 861 - 870, 2008.

Moreno-Munoz, A. y De LA Rosa, J. Voltage sag in highly automated factories. IEEE Industry Applications Society Annual Meeting, páginas 1 $-6,2008$.

Morren, J., Pierik, J. y DE HAAn, S. Inertial response of variable speed wind turbines. Electric Power Systems Research, vol. 76, no. 11, páginas $980-987,2006$.

Muyeen, S., Ali, M., Takahashi, R., Murata, T., Tamura, J., Tomaki, Y., Sakahara, A. y Sasano, E. Comparative study on transient stability analysis of wind turbine generator system using different drive train models. IET Renewable Power Generation, vol. 1, no. 2, páginas $131-141,2007$. 
Nelson, J. System grounding and ground-fault protection in the petrochemical industry: a need for a better understanding. IEEE Transactions on Industry Applications, vol. 38, no. 6, páginas 1633 - 1640, 2002.

NERC. Standard models for variable generation. Informe técnico, North American Electric Reliability Corporation, 2010.

Novak, T., Morley, L. y Trutt, F. Sensitive ground-fault relaying. IEEE Transactions on Industry Applications, vol. 24, no. 5, páginas 853 $861,1988$.

O'Connell, R. y Kirawanich, P. ATP-EMTP study of adjustable speed drive dc link properties during voltage sags. IEEE Power Engineering Society General Meeting, vol. 3, páginas 2548 - 2555, 2005.

Parmentier, R., Gruppi, D., Mussio, L., Lealeu, N., Boquet, M., Cariou, J. P., Nostrand, T. J., Heco, E. y Clarke, S. H. Lidar improvements for wind energy projects in remote sites. Eurpean Wind Energy Conference, 2010.

Paulsen, U. S., Mortensen, N. G. y Hansen, J. C. Field calibration of cup anemometers. European Wind Energy Conference, 2007.

PeñA, A. Sensing the wind profile. Tesis Doctoral, Technical University of Denmark, 2009.

Pedra, J., Corcoles, F. y SAinz, L. Study of AC contactors during voltage sags. 10th International Conference on Harmonics and Quality of Power, vol. 2, páginas 565 - 570, 2002.

Pedra, J., Corcoles, F. y Suelves, F. Effects of balanced and unbalanced voltage sags on VSI-fed adjustable-speed drives. IEEE Transactions on Power Delivery, vol. 20, no. 1, páginas 224 - 233, 2005.

Peiponen, K.-E., Myllyla, R. y Priezzhev, A. V. Optical Measurement Techniques: Innovations for Industry and the Life Sciences. Springer, 2009.

Pelletier, M., Faghani, D., Boquet, M., Dexter, R., Boucher, B., Osler, E., Masson, C. y Landberg, L. LiDAR validation in complex terrain. European Wind Energy Association. Wind Energy Event, 2011.

Perdana, A. Dynamic Models of Wind Turbines. A Contribution towards the Establishment of Standardized Models of Wind Turbines for Power System Stability Studies. Tesis Doctoral, Chalmers University of Technology, 2008. 
Petronijevic, M., Jeftenic, B., Mitrovic, N. y Kostic, V. Voltage sag drop in speed minimization in modern adjustable speed drives. IEEE International Symposium on Industrial Electronics, vol. 3, páginas 929 934, 2005.

PHILIPS. LED Lighting Explained. Understanding LED Sources, Fixtures, Applications, and Opportunities. Philips, 2010.

Pindado, S., Vega, E., Martínez, A., Meseguer, E., Franchini, S. y Pérez Sarasola, I. Analysis of calibration results from cup and propeller anemometers. influence on wind turbine annual energy production (AEP) calculations. Wind Energy, vol. 14, no. 1, páginas 119 - 132, 2011.

Pohjanheimo, P. y Lehtonen, M. Equipment sensitivity to voltage sagstest results for contactors, PCs and gas discharge lamps. 10th International Conference on Harmonics and Quality of Power, vol. 2, páginas 559 - 564, 2002.

Pourbeik, P. Model validations attempts for the type 3 generic model structure-review of proposed changes by gamesa. Informe técnico, EPRI, $2012 \mathrm{a}$.

PourbeIK, P. Proposed changes to the WECC WT3 generic model for type 3 wind turbine generators. Informe técnico, Electric Power Research Institute, 2012b.

Poza Alonso, L. El contactor y su entorno. En El mundo del automatismo. GRUDILEC, 2005.

Price, W. y Sanchez-Gasca, J. Simplified wind turbine generator aerodynamic models for transient stability studies. IEEE PES Power Systems Conference and Exposition, páginas 986 - 992, 2006.

Ramela, K. y Kumar, V. Simulation of ride through capability of adjustable speed drive for type A and type B voltage sags and well using buck-boost converter. International Conference on Recent Advancements in Electrical, Electronics and Control Engineering, páginas 506 - 511, 2011.

Ravalli, P. y Leung, J. Dynamic model requirements and model validation in the australian national electricity market. IEEE Power and Energy Society General Meeting, páginas 1 - 5, 2011.

REE. El sistema eléctrico español. Informe técnico, Red Eléctrica de España, 2012.

Riba, J.-R., Garcia, A., Cusido, J. y Delgado, M. Dynamic model for $\mathrm{AC}$ and DC contactors - simulation and experimental validation. Simulation Modelling Practice and Theory, vol. 19, no. 9, páginas 1918 - 1932, 2011. 
Rob, R., Jewell, W. y ARAR, A.-R. The effect of power quality variations on a PC. 36th Midwest Symposium on Circuits and Systems, vol. 2, páginas $1431-1432,1993$.

Rocha, B. y Madrigal, M. Analysis of performance of adjustable-speed drives during voltage sags. IEEE Latin America Transactions, vol. 3, no. 5, páginas 39 - 46, 2005.

Rodriguez Amenedo, J., Burgos Diaz, J. y Arnalte Gomez, S. Sistemas Eólicos de Producción de Energía Eléctrica. Rueda S.L., 2003.

SAIED, M. M. An approach to the assessment of voltage quality based on a modified power acceptability curve. IEEE Transactions on Power Delivery, vol. 22, no. 1, páginas 613 - 618, 2007.

Saksena, S. y Karady, S. Effects of voltage sags on household loads. IEEE Power Engineering Society General Meeting, vol. 3, páginas 2456 2461, 2005.

Salles, M. B. C., Hameyer, K., Cardoso, J. R., Grilo, A. P. y RahMANN, C. Crowbar system in doubly fed induction wind generators. Energies, vol. 3, páginas $738-753,2010$.

Sanz-Andrés, A., Cuerva, A. y Martínez-Muelas, A. Statistic analysis of large calibration series of nrg max. 40 cup anemometer in wind tunnel. European Wind Energy Conference, 2003.

Sarmiento, H. y Estrada, E. A voltage sag study in an industry with adjustable speed drives. IEEE Industry Applications Magazine, vol. 2, no. 1, páginas $16-19,1996$.

Schwartz, M. y Elliott, D. Wind shear characteristics at central plains tall towers. NREL/CP-500-40019, 2006.

SEMI. SEMI F47-0706. Specification for Semiconductor Processing Equipment Voltage Sag Inmunity., 2006.

SEyedi, M. Evaluation of the DFIG Wind Turbine Built-in Model in PS$S / E$. Proyecto Fin de Carrera, CHALMERS UNIVERSITY OF TECHNOLOGY, Göteborg, Sweden, 2009.

Shareef, H., Marzuki, N., Mohamed, A. y Mohamed, K. Experimental investigation of ac contactor ride through capability during voltage sag. 9th International Conference on Environment and Electrical Engineering (EEEIC), páginas 325 - 328, 2010a.

Shareef, H., Mohamed, A. y Marzuki, N. Analysis of personal computer ride through capability during voltage sags. Electric Power Systems Research, vol. 79, no. 12, páginas 1615 - 1624, 2009a. 
Shareef, H., Mohamed, A. y Mohamed, K. Development of voltage acceptability curves for fluorescent lighting systems. International Conference on Electrical Engineering and Informatics, vol. 2, páginas 513-518, 2009b.

Shareef, H., Mohamed, A. y Mohamed, K. Sensitivity of compact fluorescent lamps during voltage sags: An experimental investigation. WSEAS Transactions on Power Systems, vol. 5, no. 1, páginas 22 - 31, 2010b.

Short, T. A. Electric Power Distribution Handbook. CRC Press, 2004.

Sisterson, D., Hicks, B. B., Coulter, R. L. y Wesely, M. L. Difficulties in using power laws for wind energy assessment. Solar Energy, vol. 31, no. 2, páginas 201 - 204, 1983.

Smith, D. A., Harris, M., Coffey, A. S., Mikkelsen, T., Jørgensen, H. E., Mann, J. y Danielian, R. Wind lidar evaluation at the danish wind test site in høvsøre. Wind Energy, vol. 9, páginas 87 - 93, 2006.

Sørensen, P. Introduction to IEC 61400-27. Electrical simulation models for wind power generation. EERA Workshop on Generic electric models for wind power, 2012.

Sørensen, P., Andersen, B., Bech, J., Fortmann, J. y Pourbeik, P. Progress in IEC 61400 -27. Electrical simulation models for wind power generation. 11th International Workshop on Large-Scale Integration of Wind Power into Power Systems as well as on Transmission Networks for Offshore Wind Power Farms, 2012.

Sørensen, P., Andersen, B., Fortmann, J., Johansen, K. y PourBEIK, P. Overview, status and outline of the new IEC 61400-27 - Electrical simulation models for wind power generation. 10th International Workshop on Large-Scale Integration of Wind Power into Power Systems as well as on Transmission Networks for Offshore Wind Power Farms, 2011.

Stephen, O., Blae, A. y Jonhson, D. A. Experimental study of the effect of tower shadow on anemometer readings. Journal of Wind Engineering and Industrial Aerodynamics, vol. 99, no. 1, páginas 1-6, 2011.

Stephens, M. PQ in Continuous Manufacturing. Informe técnico, Leonardo Energy, 2007.

Stockman, K., D'hulster, F., Verhaege, K., Didden, M. y Belmans, R. Ride-through of adjustable speed drives during voltage dips. Electric Power Systems Research, vol. 66, no. 1, páginas 49 - 58, 2003. ISSN 0378-7796. 
Tarczynski, W., Hejman, T. y Smugala, D. Computer-controlled testing system for investigating the dynamic characteristics of contactors with A.C. electromagnet drives. Measurement, vol. 33, no. 4, páginas 313 - 323, 2003.

Thet, A. K. y SAitoh, H. Pitch control for improving the low-voltage ridethrough of wind farm. Transmission Distribution Conference Exposition: Asia and Pacific, páginas $1-4,2009$.

Timbus, A., Korba, P., Vilhunen, A., Pepe, G., Seman, S. y NiiraNEN, J. Simplified model of wind turbines with doubly-fed induction generator. 10th International Workshop on Large-Scale Integration of Wind Power into Power Systems as well as on Transmission Networks for Offshore Wind Power Farms, 2011.

TPWIND. Strategic research agenda. Market deployment strategy. From 2008 to 2030. Informe técnico, European Technology Platform for Wind Energy, 2008.

Turner, E. y Collins, E. The performance of AC contactors during voltage sags. International Conference on Harmonics and Quality of Power, 1996.

UNE-EN 60947-4-1. UNE-EN 60947-4-1: Aparamenta de baja tensión. parte 4-1: Contactores y arrancadores de motor. contactores y arrancadores electromecánicos. 2011.

UNE-EN 61000-4-11. UNE-EN 61000-4-11: Compatibilidad electromagnética (cem). parte 4-11: Técnicas de ensayo y medida. ensayos de inmunidad a los huecos de tensión, interrupciones breves y variaciones de tensión. 2005.

UNE-EN 61547. UNE-EN 61547: Equipos para iluminación para uso general. Requisitos relativos a la inmunidad CEM. 2011.

Uski-Joutsenvuo, S. y Niskanen, S. Wind turbine models - status report of model development and verification measurements. Informe técnico, VTT Technical Research Centre of Finland, 2012.

Vegunta, S. y Milanovic, J. Estimation of cost of downtime of industrial process due to voltage sags. IEEE Transactions on Power Delivery, vol. 26, no. 2, páginas $576-587,2011$.

Vegunta, S. C., Anaya-lara, O., Milanovic, J. V. y Jenkins, N. Sensitivity of active front-end ASDs and DFIGs to voltage sags and short interruptions. The 3rd IET International Conference on Power Electronics, Machines and Drives., páginas 637 - 641, 2006. 
WAGNER, R. Accounting for the speed shear in wind turbine power performance measurement. Tesis Doctoral, Technical University of Denmark, Roskilde, Denmark, 2010.

Wagner, R., Antoniou, I., Pedersen, S. M., Courtney, M. S. y JorGensen, H. E. The influence of the wind speed profile on wind turbine performance measurements. Wind Energy, vol. 12, páginas 348 - 362, 2009.

Wagner, R., Courtney, M., Gottschall, J. y Lindelow-Marsden, P. Improvement of power curve measurement with lidar wind speed profiles. European Wind Energy Conference, 2010.

Wagner, R., Jorgensen, H. E., Paulsen, U., Larsen, T. J., AntoNiou, I. y ThesbJerg, L. Remote sensing used for power curves. 14th International Symposium for the Advancement of Boundary Layer Remote Sensing, 2008.

WECC REMTF. WECC wind power plant dynamic modeling guide. Informe técnico, WECC, 2010.

Weitkamp, C. Lidar: Range-Resolved Optical Remote Sensing of the Atmosphere. Springer, 2005.

Won, D.-J., Ahn, S.-J., Chung, I.-Y., Kim, J.-M. y Moon, S.-I. A new definition of voltage sag duration considering the voltage tolerance curve. IEEE Bologna Power Tech Conference, vol. 3, 2003.

Wong, K., Ho, S. y Cheng, K. Minimization of current stress on the grid synchronization of doubly-fed induction generators for wind power generation. 3rd International Conference on Power Electronics Systems and Applications, páginas 1 - 4, 2009.

WWEA. 2012 half-year report. Informe técnico, World Wind Energy Association, 2012.

Zavadil, R., Miller, N., Ellis, A. y Muljadi, E. Making connections: Wind generation challenges and progress. IEEE Power and Energy Magazine, vol. 3(6), páginas 26-37, 2005.

Zhang, J., Cheng, M., Chen, Z. y Fu, X. Pitch angle control for variable speed wind turbines. Third International Conference on Electric Utility Deregulation and Restructuring and Power Technologies, páginas 2691 2696, 2008.

Zielichowski, M. y SzLeZaK, T. Ground-fault protection of four-pole generators with two parallel branches in phase winding. IEEE Transactions on Power Delivery, vol. 22, no. 2, páginas 813 - 819, 2007. 
Zubiaur, R., Martínez, R., Pinedo, J. y Ortiz, D. Remote sensing. The value of the availability. European Wind Energy Association. Wind Energy Event, 2012. 



\section{Lista de Acrónimos}

ASD Adjustable Speed Drive

CBEMA Computer Business Equipment Manufacturer's Associattion

CD Committee Draft

CDV Committee Draft for Voting

CENER Centro Nacional de Energías Renovables

CFL Lámpara Fluorescente Compacta

CT Current Transformer

DFIG Doubly Fed Induction Generator

EPRI Electric Power Research Institute

HM Halogenuros Metálicos

IDAE Instituto para la Diversificación y Ahorro de la Energía

IEC International Electrotechnical Commission

IEEE Institute of Electrical and Electronics Engineers

ITI Information Technology Industry Council

IWES Fraunhofer Institute for Wind Energy and Energy System Techno$\log y$

LED Light Emiting Diode

LFL Lámpara Fluorescente Lineal

LiDAR Light Detection And Ranging

MEASNET International Network for Harmonised and Recognised Measurements in Wind Energy

NERC North American Electric Reliability Corporation

PC Personal Computer

REE Red Eléctrica de España

REMTF Renewable Energy Modeling Task Force

RMSE Root Mean Square Error 
SAI Sistema de Alimentación Ininterrumpida

SCADA Supervisory Control And Data Acquisition

SoDAR Sound Detection And Ranging

TPWIND European Technology Platform for Wind Energy

UPS Uninterruptible Power Supply

VFD Variable Frequency Drive

VSAP Vapor de Mercurio a Alta Presión

VSAP Vapor de Sodio a Alta Presión

WECC Western Electricity Coordinating Council

WGMG Wind Generator Modeling Group 
